\title{
Untersuchung der
}

\section{Versetzungsnukleation in}

\section{Gold-Nanodrähten durch in-situ Elektronenmikroskopie}

\author{
Dissertation \\ zur Erlangung des mathematisch-naturwissenschaftlichen Doktorgrades \\ „Doctor rerum naturalium“ \\ der Georg-August-Universität Göttingen \\ im Promotionsprogramm ProPhys \\ der Georg-August University School of Science (GAUSS)
}

vorgelegt von

Bahne Kapelle

aus Heide (Holst.)

Göttingen, 2016 


\section{Betreuungsausschuss:}

Prof. C. A. Volkert

Institut für Materialphysik

Georg-August-Universität Göttingen

Prof. M. Seibt

IV. Physikalisches Institut - Halbleiterphysik

Georg-August-Universität Göttingen

\section{Mitglieder der Prüfungskommission:}

$\underline{\text { Referentin: }}$

Prof. C. A. Volkert

Institut für Materialphysik

Georg-August-Universität Göttingen

Korreferent:

Prof. M. Seibt

IV. Physikalisches Institut - Halbleiterphysik

Georg-August-Universität Göttingen

Weitere Mitglieder der Prüfungskommission:

Prof. H. C. Hofsäss

II. Physikalisches Institut - Kern- und Atomphysik

Georg-August-Universität Göttingen

Prof. H.-U. Krebs

Institut für Materialphysik

Georg-August-Universität Göttingen

Prof. V. Moshnyaga

I. Physikalisches Institut - Tieftemperaturphysik

Georg-August-Universität Göttingen

Prof. W. Kollatschny

Institut für Astrophysik

Georg-August-Universität Göttingen

Tag der mündlichen Prüfung: 12.02.2016 


\section{Inhaltsverzeichnis}

1. Einleitung 3

$\begin{array}{ll}\text { 2. Theoretische Grundlagen } & 7\end{array}$

2.1. Grundlagen der Verformung . . . . . . . . . . . . . . . . . . . . 7

2.2. Aspekte zur Versetzungsnukleation . . . . . . . . . . . . . . . . 9

2.2.1. Aufgelöste Schubspannung . . . . . . . . . . . . . . . . . 11

2.2.2. Generalisierte Stapelfehlerenergie . . . . . . . . . . . . . 12

2.2.3. Oberflächengeometrie . . . . . . . . . . . . . . . . . 15

2.3. Klassische Keimbildung . . . . . . . . . . . . . . . . . . . . . . . 16

2.4. Fließgrenze bei Versetzungsnukleation . . . . . . . . . . . . . . . . . . . 19

2.5. Größeneffekte bei der Versetzungsnukleation . . . . . . . . . . . . . . 20

3. Experimentelle Methoden 23

3.1. Verwendete Geräte . . . . . . . . . . . . . . . . . . . . . . 23

3.1.1. Rasterelektronenmikroskop (REM) . . . . . . . . . . . . 23

3.1.2. Ionenstrahlmikropskop (FIB) . . . . . . . . . . . . . . 24

3.1.3. Transmissionselektronenmikropskop (TEM) . . . . . . . . . 24

3.2. Zugapparaturen . . . . . . . . . . . . . . . . 25

3.2.1. TEM-Aufbau . . . . . . . . . . . . . . 25

3.2.2. REM Aufbau . . . . . . . . . . . . . . . . . 26

3.3. Proben, Transfer und Ablauf der Versuche . . . . . . . . . . . . . . . . 28

3.3.1. Ablauf im TEM . . . . . . . . . . . . . . . . 30

3.3.2. Ablauf und Messgrößen im REM . . . . . . . . . . . . . . 30

3.4. Erzeugen von Defekten . . . . . . . . . . . . . . . . . 36

3.4.1. Biegen und Quetschen ... . . . . . . . . . . 37

3.4.2. 3-Punkt-Biegung . . . . . . . . . . . . . . . 38 
4. Experimentelle Ergebnisse 41

4.1. Charakterisierung der Proben . . . . . . . . . . . . . . . . 41

4.1.1. Einkristalline Proben . . . . . . . . . . . . . . . . . . 41

4.1.2. Proben mit longitudinaler Zwillingsgrenze . . . . . . . . . . . 43

4.2. Verformungsverhalten einkristalliner Drähte . . . . . . . . . . . 46

4.2.1. Planare Defekte . . . . . . . . . . . . . . . 46

4.2.2. Einfluss von anfänglichen Defekten . . . . . . . . . . . . 54

4.2.3. Verhalten bei Entlastung . . . . . . . . . . . . . . . 58

4.2.4. Bruchmorphologie und Bruchverhalten . . . . . . . . . . 60

4.2.5. Abschätzung der Dehnung . . . . . . . . . . . 66

4.3. Verformungsverhalten von Drähten mit longitudinalen Zwillingsgrenzen $\quad 73$

4.3.1. Planare Defekte und Versetzungen . . . . . . . . . . . . 73

4.3.2. Einfluss anfänglicher Defekte . . . . . . . . . . . 75

4.3.3. Verhalten bei Entlastung . . . . . . . . . . . . . . 77

4.3.4. Versagen und Bruchmorphologie . . . . . . . . . . . 79

4.4. In-situ REM Versuche . . . . . . . . . . . . . . . . . . 85

4.4.1. Dehnung . . . . . . . . . . . . . . . 85

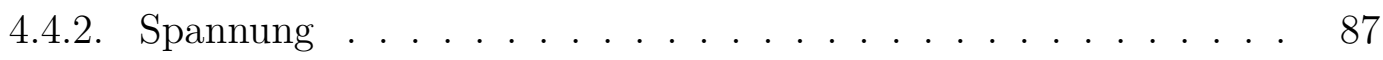

4.4.3. Spannungs-Dehnungs-Kurven . . . . . . . . . . . . . 88

4.4.4. Ergebnisse der Spannungs-Dehnungs-Kurven . . . . . . . . . . . 103

5. Diskussion 111

5.1. Elastisches Verhalten . . . . . . . . . . . . . . . . 111

5.2. Nukleationskontrollierte Verformung . . . . . . . . . . . . . . . 114

5.2.1. Nukleation von leading-Partialversetzungen . . . . . . . . . . 119

5.2.2. Nukleation von twinning-Partialversetzungen . . . . . . . . . . 122

5.2.3. Nukleation von trailing-Partialversetzungen . . . . . . . . . 127

5.3. Wechselwirkung von Partialversetzungen mit weiteren Defekten . . . . 132

5.4. Speicherung von Versetzungen . . . . . . . . . . . . . . . 136

5.5. Bruchverhalten . . . . . . . . . . . . . . . 137

5.5.1. Plastische Dehnung bis zum Versagen . . . . . . . . . . . . 138

5.5.2. Verschwinden von planaren Defekten beim Reißen . . . . . . . . 139

$\begin{array}{ll}\text { 6. Zusammenfassung } & 143\end{array}$ 
A. Einfluss der Platinschicht

B. Einfluss des Elektronenstrahls

151

C. Zugversuche an Kupfer- und Silberdrähten

153

C.1. Ergebnisse Kupfer . . . . . . . . . . . . . . . . . . . . . . . 153

C.2. Ergebnisse Silber . . . . . . . . . . . . . . . . . 156

C.3. Fazit . . . . . . . . . . . . . . . . . . 158

$\begin{array}{ll}\text { Literaturverzeichnis } & 159\end{array}$ 



\section{Einleitung}

Metalle finden seit jeher eine vielseitige und vielfältige Anwendung als Werkstoff in unterschiedlichsten Bereichen. Insbesondere deren mechanische Eigenschaften und das Verformungsverhalten sind hierbei von zentraler Bedeutung. Mit zunehmendem Trend zur Miniaturisierung und Nanotechnologie auf vielen Entwicklungsfeldern ist deshalb ein grundlegendes Verständnis der mechanischen Eigenschaften und der Verformungsmechanismen auf eben diesen nanoskaligen Längenskalen unabdingbar, um mit diesem Wissen die mechanischen Eigenschaften zukünftiger Entwicklungen gezielt auf ihre Anwendung zu gestalten.

Das Zentrum zum Verständnis für die plastische Verformung von kristallinen Materialien wie Metallen sowie deren mechanische Eigenschaften wie Festigkeit und Duktilität bilden Versetzungen. Versetzungen sind in bulk-Kristallen prinzipiell durch die Synthese oder eine anschließende Behandlung vorhanden. Eine plastische Verformung erfolgt dann durch die Aktivierung und Wechselwirkung der vorhandenen Versetzungen untereinander oder mit anderen mikrostrukturellen Eigenschaften. Dies hat zur Folge, dass Metalle im Allgemeinen eine hohe Duktilität aufweisen, zugleich aber auch eine Festigkeit besitzen, die lediglich ein Bruchteil der theoretischen Festigkeit beträgt.

Für nanostrukturierte und nanoskalige Metalle ist lange bekannt, dass sich die mechanischen Eigenschaften von ihren bulk-Gegenstücken unterscheiden. So hat Brenner bereits Mitte letzten Jahrhunderts in seinen bahnbrechenden Versuchen eine Zunahme der Festigkeit mit abnehmendem Durchmesser in verschiedenen fiberähnlichen fcc-Metallen, sogenannten Whiskern, feststellen können, die eine Festigkeit nahe der theoretischen Festigkeit erreichen [1][2][3][4]. Obwohl noch keine Mikrostrukturuntersuchungen durchgeführt wurden, deutet das Spannungs-Dehnungs-Verhalten der Whisker auf ein Verformungsverhalten kontrolliert durch die Nukleation von Versetzungen an Oberflächenfehlern hin.

Neben einer Zunahme der Festigkeit ist in jüngerer Zeit ebenfalls erkannt worden, 
dass eine Verformung kontrolliert durch die Wechselwirkung von vollen Versetzungen scheinbar auf der Nanoebene weniger von Bedeutung ist. Dies ist dadurch begründet, dass auf der einen Seite nur wenige Versetzungen in nanoskaligen Proben vorhanden sind. Auf der anderen Seite kommt hinzu, dass durch die große Oberfläche im Vergleich zum Volumen Versetzungen einfach aus der Probe herauslaufen können [5], ohne dass es zu einer Wechselwirkung kommt und damit keine selbsterhaltende Population von Versetzungen in der Probe verbleibt [6][7]. Die Verformung ist dann bestimmt durch die Aktivierung von Versetzungsquellen, z.B. Frank-Read-Quellen oder Spiralarmquellen [8][9] oder im Fall kompletter Abwesenheit von Versetzungen durch die Nukleation von neuen Versetzungen [10].

Somit stellen sich die zentralen Fragen für die Plastizität auf der Nanoskala:

1. Wie unterscheidet sich eine Verformung basierend auf der Nukleation von Versetzungen von der herkömmlichen Verformung über die Aktivierung und Wechselwirkung von vorhandenen Versetzungen?

2. Unter welchen Bedingungen kommt es zu einem Wechsel im Verformungsmechanismus und zur Verformung via Nukleation von neuen Versetzungen?

Für die vorliegenden Arbeit wurde das Nukleationsverhalten von Versetzungen in einkristallinen sowie verzwillingten Nanodrähten untersucht, um das bisherige Verständnis einer nukleationsbasierten Verformung zu überprüfen und zu erweitern. Um einen möglichst einfachen Spannungszustand zu gewährleisten, wurden die Drähte unter Zug getestet. Auf der einen Seite erfolgten die Untersuchungen in-situ im Transmissionselektronenmikroskop (TEM), die im Rahmen der Arbeit von Burkhard Roos [11] begonnen und im Zuge dieser Arbeit fortgeführt wurden. Hierdurch war es möglich, die Defekt-Evolution direkt zu beobachten und damit so nah wie möglich an den Nukleationsprozess zu gelangen. Um hierfür einen möglichst stabilen Aufbau zur Verfügung zu haben und etwaige Instabilitäten durch eine Nachgiebigkeit der Testapparatur zu vermeiden, wurde in den in-situ TEM Untersuchungen komplett auf die Messung von Spannungen verzichtet. Auf der anderen Seite kam für die quantitative Analyse der Spannung und Dehnung ein Aufbau im Rasterelektronenmikroskop zum Einsatz, der im Zuge meiner Diplomarbeit [12] entwickelt und im Verlauf dieser Arbeit sowie in Rahmen verschiedener Bachelorarbeiten [13][14][15] kontinuierlich weiterentwickelt wurde. Mit post-mortem Analysen der Mikrostruktur ließ sich eine Verbindung zu den 
in-situ TEM Untersuchungen herstellen, so dass sich ein relativ komplettes Bild der Verformung ergab.

\section{Aufbau der Arbeit}

Im Anschluss an diese Einleitung werden im folgenden Kapitel die physikalischen Grundlagen dieser Arbeit beleuchtet. Im Fokus stehen hierbei die zentralen Aspekte der Versetzungsnukleation sowie Vorhersagen und Modelle für eine nukleationsbasierte Verformung. Im dritten Kapitel erfolgt die Vorstellung der experimentellen Methoden. Hierzu gehört ein kurzer Abriss der verwendeten Geräte. Nachfolgend werden die beiden verwendeten Zugapparaturen sowie die Probenpräparation und der Ablauf der Versuche beschrieben. Das vierte Kapitel beinhaltet die Erörterung der experimentellen Ergebnisse dieser Arbeit. Dabei werden zunächst kurz die Proben charakterisiert. Anschließend folgt die Darstellung der Ergebnisse der Mikrostruktur-Untersuchungen gegliedert nach einkristallinen Proben und Proben mit longitudinaler Zwillingsgrenze. Abgeschlossen wird das Kapitel mit der Darstellung der Spannungs-Dehnungs-Kurven und den daraus gewonnenen Größen. Das fünfte Kapitel ist der Diskussion der Ergebnisse gewidmet und im sechsten Kapitel wird schließlich eine Zusammenfassung gegeben. 



\section{Theoretische Grundlagen}

Gegenstand dieser Arbeit ist die Untersuchung der mechanischen Eigenschaften von Gold-Nanodrähten und deren Verformung via Versetzungsnukleation. Hierzu werden in diesem Kapitel zunächst die Grundlagen der Verformung vorgestellt. Anschließend folgt die Erörterung der wichtigsten Aspekte der Versetzungsnukleation und der klassischen Keimbildung im Kontext der Versetzungsnukleation.

\subsection{Grundlagen der Verformung}

Setzt man Festkörper einer externen Kraft aus, so haben sie die Eigenschaft, ihre Form zu verändern. Bis zu einem gewissen Limit kehren Festkörper zurück in ihre Ursprungsform, sobald die äußere Kraft wieder zurückgenommen wird. Dieses reversible Verhalten wird als Elastizität bezeichnet. Für kleine Verformungen wird der Zusammenhang mit dem Spannungszustand, der durch die äußere Kraft bei reiner Zug- oder Druckbelastung auf den Festkörper hervorgerufen wird, durch das Hookesche Gesetz in seiner einfachsten Form beschrieben:

$$
\sigma_{x}=E \epsilon_{x}
$$

$E$ ist das Elastizitätsmodul. Dieser lineare Zusammenhang stammt von dem quadratischen Term der potentiellen Energie der interatomaren Bindungen und ist daher nur gültig für kleine Auslenkungen. Für große Auslenkungen sind Terme höherer Ordnung zu berücksichtigen $\left(\sigma=E \epsilon+E_{2} \epsilon^{2}+\ldots\right)$.

Wird das elastische Limit überschritten, erfährt der Festkörper eine permanente Verformung. Diese Verformung ist irreversibel und wird als Plastizität bezeichnet. Der Mechanismus für die plastische Verformung von Kristallen ist im Allgemeinen die Bewegung von Versetzungen. Jede Versetzung, die sich vollständig durch den Kristall bewegt, führt zu einer Abscherung des Kristalls entlang der Gitterebene der Verset- 
zung um den Burgersvektor $b$ der Versetzung. Um die Bewegung von Versetzungen zu aktivieren, muss das periodische Peierls-Potential, das durch die Atome auf ihren Gitterplätzen gebildet wird, überwunden werden. Die dafür notwendige Peierls-Spannung $\tau_{p}$ lässt sich näherungsweise durch

$$
\tau_{p}=\frac{2 \mu}{1-\nu} \exp \left(-\frac{2 \pi}{(1-\nu)} \frac{d}{b}\right)
$$

beschreiben. $\mu$ ist hierbei das Schermodul und $\nu$ die Querkontraktionszahl. Wie man an Gleichung 2.2 erkennen kann, hängt die Peierls-Spannung in hohem Maße vom Verhältnis zwischen dem Gitterebenenabstand $d$ und dem Burgersvektor $b$ ab. Um eine möglichst kleine Peierls-Spannung überwinden zu müssen, erfolgt die Bewegung von Versetzungen daher auf Ebenen mit einem großen Abstand zu einander und in Richtung eines kleinen Burgersvektors. Für kubisch flächenzentrierte Kristalle (engl.: face centered cubic; kurz: fcc) sind dies die vier $\{111\}$-Ebenen, in denen die drei $\langle 110\rangle$ Richtungen den kleinsten Burgersvektor aufweisen. Für fcc-Kristalle existieren also zwölf (4 x 3) Gleitsysteme aus Gleitebene und Gleitrichtung, auf denen die Bewegung der Versetzungen maßgeblich stattfindet.

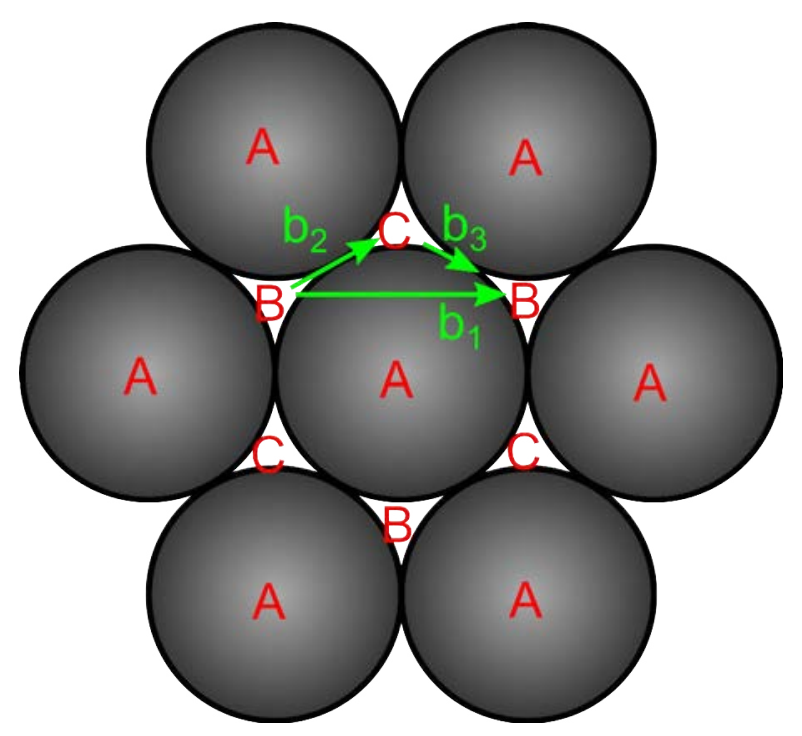

Abbildung 2.1.: Aufspaltung einer Versetzung in zwei Partialversetzungen

Jede Versetzung führt zu einer Verzerrung des regulären Gitters und die zugehörige Verzerrungsenergie bzw. Linienenergie $E_{L}$ einer Versetzung kann durch

$$
E_{L}=L \cdot \frac{(2-\nu)}{(1-\nu)} \frac{\mu b^{2}}{8 \pi}\left(\ln \frac{4 \alpha r}{b}-2\right)
$$


beschrieben werden[16]. $L$ ist dabei die Länge der Versetzung und $\alpha$ beschreibt den Kern der Versetzung. Eine Versetzung kann ihre Energie dadurch verringern, dass sie sich in zwei Partialversetzungen aufspaltet. Für fcc-Kristalle ist ein solcher Fall bei der Aufspaltung einer vollen Versetzung in zwei Shockley-Partialversetzungen gemäß

$$
\begin{aligned}
b_{1} & \rightarrow b_{2}+b_{3} \\
a / 2[1 \overline{1} 0] & \rightarrow a / 6[2 \overline{11}]+a / 6[1 \overline{1} 1] \\
E_{L, \text { volle }} \approx \mu a^{2} / 2 & >\mu a^{2} / 3 \approx 2 \cdot E_{L, \text { partial }}
\end{aligned}
$$

gegeben (siehe Abbildung 2.1). Bei den Burgersvektoren von Partialversetzungen handelt es sich jedoch nicht um Translationsvektoren des Gitters. Hierbei wird das Kristallgitter in der Weise gestört, dass sich die Stapelreihenfolge ABCABC der $\{111\}$-Ebenen ändert und ein Stapelfehler entsteht.

\subsection{Aspekte zur Versetzungsnukleation}

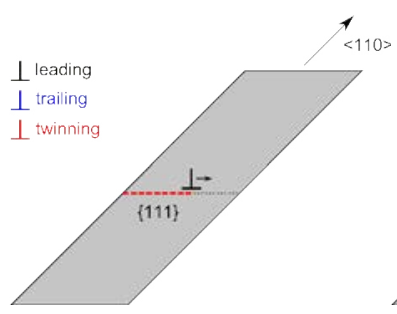

(a)

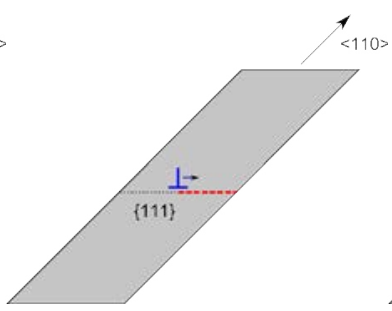

(b)

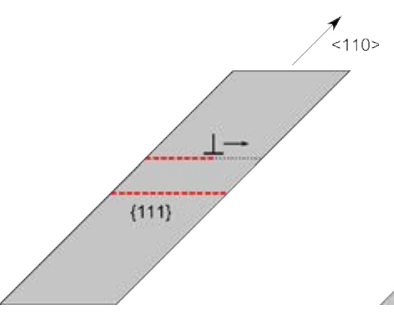

(c)

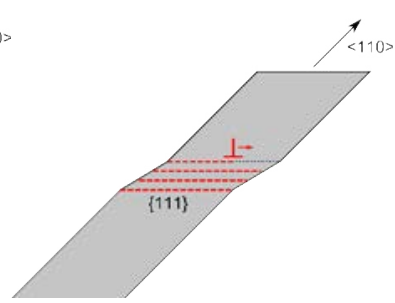

(d)

Abbildung 2.2.: Schematische Skizzen zu den möglichen Nukleationen von Partialversetzungen: (a) Nukleation einer leading-Partialvertzung, die einen Stapelfehler hinterlässt, (b) mögliches Szenario der Nukleation einer trailing Partialversetzung, die den Stapelfehler annihiliert, (c) mögliches Szenario der Nukleation einer weiteren leading-Partialversetzung, so dass ein zweiter Stapelfehler entsteht, (d) mögliches Szenario der Nukleation von twinning-Partialversetzungen auf benachbarten Ebenen des Stapelfehlers, wodurch sowohl ein Zwilling als auch eine Reorientierung des Drahtes ensteht.

In Nanodrähten, die frei von Versetzungen sind, sind die typischen Versetzungsquellen wie z.B. Frank-Read-Quellen nicht vorhanden, so dass die Versetzungsnukleation eine entscheidende Rolle in der plastischen Verformung spielen muss. Laut 
klassischer Nukleationstheorie und durch viele MD-Simulationen bestätigt ist der erste Schritt bei der Versetzungnukleation für fcc-Metalle die Nukleation einer leadingPartialversetzung [17][18][19], was schematisch für den Fall eines Drahtes mit $\langle 110\rangle$ Achse in Abbildung 2.2 (a) skizziert ist. Bei der Bewegung der Partialversetzung hinterlässt diese in ihrer Spur einen Stapelfehler.

Für die weitere Verformung existieren verschiedene Szenarien [17][18][19]. Folgt der leading-Partialversetzung die Nukleation einer trailing-Partialversetzung auf der gleichen Ebene, so entspricht dies der Verformung über eine volle Versetzung. Der Stapelfehler der leading-Partialversetzung wird dabei durch die trailing-Partialversetzung wieder annihiliert (siehe Abbildung 2.2 (b)). Sofern die trailing-Partialversetzung der leading-Partialversetzung instantan folgt, würde bei Nanodrähten lediglich eine Oberflächenstufe entstehen, welche experimentell schwer nachzuweisen ist. Folgt die Nukleation der trailing-Partialversetzung zeitlich verzögert, ließe sich zunächst der Stapelfehler der leading-Partialversetzung beobachten, welcher anschließend wieder verschwindet.

Ein anderes Szenario stellt sich so dar, dass der ersten leading-Partialversetzung die Nukleation einer weiteren leading-Partialversetzung auf einer parallelen Ebene (bzw. aus Symmetriegründen auf einer gleichberechtigten Ebene) folgt. Findet die Nukleation auf einer Ebene statt, die nicht an den ersten Stapelfehler angrenzt, so entspricht dies einer Verformung über Partialversetzung, bei der separierte Stapelfehler entstehen (Abbildung 2.2 (c)). Handelt es sich bei der Ebene um eine benachbarte Ebene, so entsteht zunächst ein extrinsischer Stapelfehler. Bei der weiteren Nukleation von Partialversetzungen auf benachbarten Ebenen kommt es dann zur Verformung über eine Zwillingsbildung (engl. twinning). Die Nukleation einer leading-Partialversetzung auf einer benachbarten Ebene wird daher als twinning-Partialversetzung bezeichnet.

Welche Art der Verformung und damit der Versetzungsnukleation abläuft, ist ein komplexer Prozess und wird durch verschiedene Faktoren beeinflusst. Nach Weinberger und Cai [19] gibt es mehrerer Schlüssel-Aspekte, die die Art der Versetzungsnukleation beeinflussen. Mit Hilfe dieser Faktoren lassen sich Vorhersagen treffen, welche Art der Versetzungsnukleation eintritt. Hierzu gehören:

- Aufgelöste Schubspannung

- Generalisierte Stapelfehlerenergie

- Oberflächengeometrie 


\subsubsection{Aufgelöste Schubspannung}

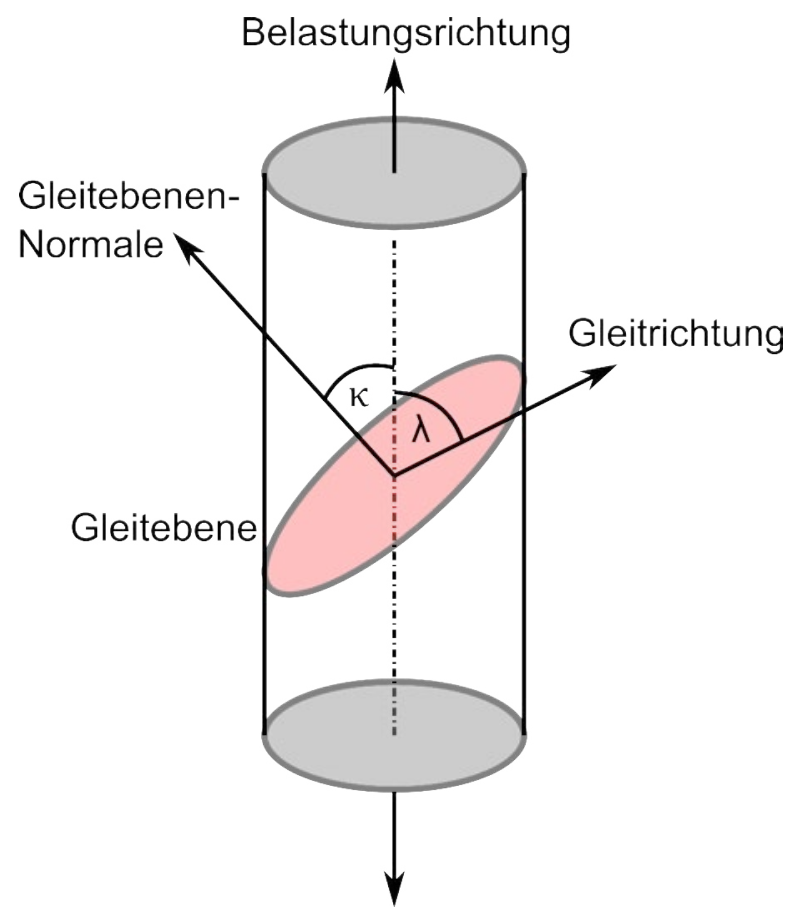

Abbildung 2.3.: Schematische Zeichnung zur Veranschaulichung des Schmidfaktors: $\lambda$ ist der Winkel zwischen Gleitrichtung und Belastungsrichtung und $\kappa$ der Winkel zwischen der Gleitebenennormalen und der Belastungsrichtung

In bulk-fcc-Metallen beginnt die plastische Verformung, wenn die Schubspannung auf ein Gleitsystem einen kritischen Wert für die Aktivierung von bereits vorhandenen Versetzungen überschreitet. Bei uniaxialer Belastung kann das Gleitsystem, das als erstes diese kritische Schubspannung erreicht, einfach durch geometrische Gegebenheiten mit Hilfe des Schmidfaktors $m_{S}$ bestimmt werden:

$$
m_{S}=\cos \kappa \cdot \cos \lambda
$$

$\lambda$ ist hierbei der Winkel zwischen der Gleitrichtung und der Belastungsrichtung und $\kappa$ der Winkel zwischen Gleitebenennormalen und Belastungsrichtung (siehe Abbildung 2.3). Das Gleitsystem mit dem höchsten Schmidfaktor $\left(0 \leq m_{S} \leq 0,5\right)$ erfährt also auch die höchste Schubspannung und wird folglich als erstes aktiviert. Die Art der Verformung (Aktivierung von bereits vorhandenen Versetzungen) ist jedoch unabhängig vom Schmidfaktor.

Im Fall der Versetzungsnukleation in Nanodrähten haben MD Simulationen ge- 
zeigt, dass neben der Selektion des Gleitsystems auch die Art der Verformung sehr stark von der Orientierung des Drahtes und der Art der Belastung (Zug- oder Druckverformung) abhängt. Welches Szenario der Versetzungsnukleation eintritt, kann nach Park und Zimmermann [18] durch eine einfache Betrachtung der Schmidfaktoren für die leading- und trailing-Partialversetzung auf den $\{111\}$-Ebenene vorhergesagt werden. Sofern der Schmidfaktor der trailing-Partialversetzung größer ist als der der leading-Partialversetzung, wird anschließend direkt die Nukleation der trailingPartialversetzung erwartet, was einer Verformung über volle Versetzungen entspricht. Ist dagegen der Schmidfaktor der leading-Partialversetzung größer als der Schmidfaktor der trailing-Partialversetzung, ist die Nukleation einer trailing-Partialversetzung nicht zu erwarten und die Verformung findet ausschließlich über die Nukleation von leading-Partialversetzungen statt.

Im Fall von Drähten mit einer $\langle 110\rangle$-Orientierung ist der Schmidfaktor der leading-Partialversetzung $(\mathrm{m}=0,47)$ doppelt so groß wie der Schmidfaktor der trailingPartialversetzung $(\mathrm{m}=0,23)$. Demnach ist nach diesem einfachen Modell eine Verformung über leading-Partialversetzungen zu erwarten. Dieses einfache Modell zeigt eine gute Übereinstimmung mit vielen Simulationen [18] und auch Experimenten [20][21][22]. Allerdings lässt sich hiermit nicht unterscheiden, ob die Verformung über twinning- oder separierte leading-Partialversetzungen abläuft.

\subsubsection{Generalisierte Stapelfehlerenergie}

Der intrinsische Materialparameter, welcher die Versetzungsnukleation hauptsächlich beeinflusst, ist die Stapelfehlerenergie. Die Stapelfehlerenergie ist jedoch nicht als absoluter Wert aufzufassen, vielmehr ist sie als Kurve der generalisierten Stapelfehlerengie (GSF) zu betrachten [24]. Die GSF-Kurve repräsentiert den Energieverlauf zweier starr gegeneinander verschobener Kristallhälften auf einer $\{111\}$-Ebene entlang der $\langle 112\rangle$ Richtung und wurde zuerst von Vitek [25] eingeführt. Die rote Kurve in Abbildung 2.4 (a) zeigt den Energieverlauf der GSF für eine leading-Partialversetzung. Entlang der Kurve muss das System zunächst ein Maximum überwinden, welches als instabile Stapelfehlerenergie $\gamma_{u S F}$ bezeichnet wird. Bei einer Verschiebung, die dem Burgersvektor einer leading-Partialveretzung entspricht, durchläuft die GSF-Kurve ein Minimum mit der Stapelfehlerenergie $\gamma_{i S F}$, die der Energie eines intrinsischen Stapelfehlers entspricht.

Rice [26] hat das Prinzip der GSF-Kurve zur Beschreibung der Nukleation ei- 


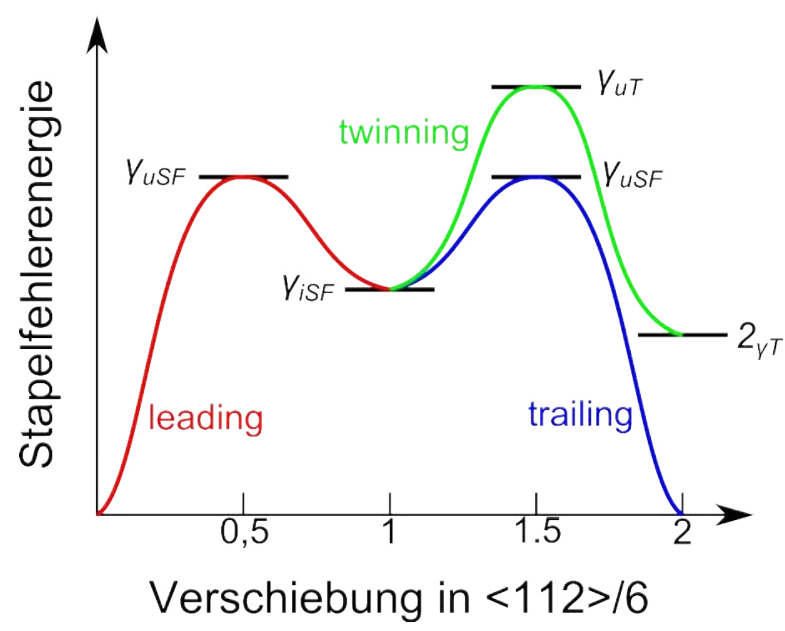

(a)

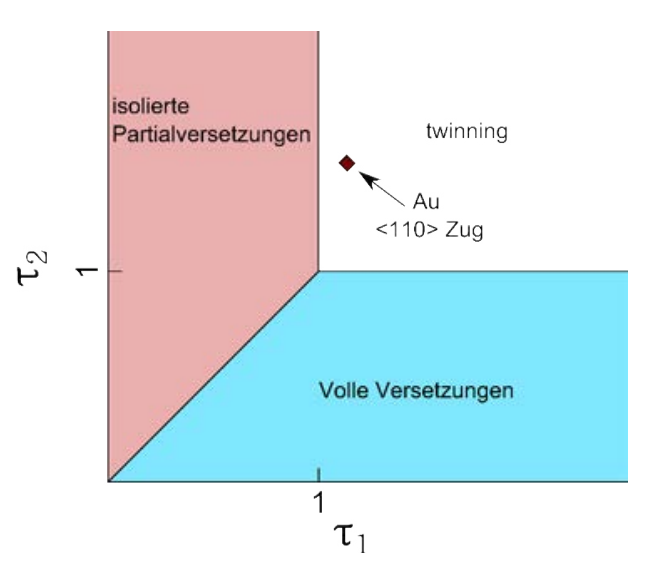

(b)

Abbildung 2.4.: GSF Vorhersage der Verformung: a) Energieverlauf der GSF-Kurve für die leading-Partialversetzung (rote Kurve), anschließender twinningPartialversetzung auf einer benachbarten Ebene (grüne Kurve) oder eine trailing-Partialversetzung (blaue Kurve) (nach [23]); b) ZweiParameter-Karte für den Verformungsmechanismus (nach [19])

ner Versetzung an einer Rissspitze verwendet und $\gamma_{u S F}$ als die Energiebarriere für die Nukleation einer Partialversetzung interpretiert. Darüber hinaus hat er das Bild der GSF-Kurve für eine trailing-Partialversetzung erweitert (blaue Kurve in Abbildung 2.4 (a)). Dabei geht er von einem Kristall aus, in dem bereits ein intrinsischer Stapelfehler vorhanden ist und die Verschiebung nun entlang einer anderen $\langle 112\rangle$-Richtung erfolgt, die einen Winkel von $60^{\circ}$ zur Richtung der leading-Partialversetzung bildet. Im Verlauf der GSF-Kurve für die trailing-Partialversetzung muss das System erneut ein Maximum $\gamma_{u S F}$ durchlaufen, welches identisch ist mit dem Maximum der leading-Partialversetzung. Rice konnte damit zeigen, dass die Nukleation der trailingPartialversetzung von der Differenz $\gamma_{u S F}-\gamma_{i S F}$ abhängt.

Tadmor und Hai [23] haben das Bild von Rice zusätzlich für eine twinningPartialversetzung erweitert. Dabei gehen sie ebenfalls von einem Kristall mit einem intrinschen Stapelfehler aus. Die Verschiebung erfolgt hierbei auf einer benachbarten $\{111\}$-Ebene entlang der ursprünglichen $\langle 112\rangle$-Richtung. Die GSF-Kurve für die twinning-Partialversetzung ist in Abbildung 2.4 (a) grün dargestellt. Sie durchläuft ein Maximum mit der Energie $\gamma_{u T}$ und führt schließlich zu einer Konfiguration mit einer Energie von $2 \gamma_{T}$, was dem Energiewert von zwei Zwillingsgrenzen entspricht. Analog zu den Ergebnissen von Rice konnten Tadmor und Hai so zeigen, dass die Nukleation 
einer twinning-Partialversetzung von dem Verhältnis $\gamma_{u T} / \gamma_{i S F}$ abhängt.

Mit dem Bild der GSF-Kurve kann nun mit Hilfe der materialspezifischen Parametern $\gamma_{u T}, \gamma_{u S F}$ und $\gamma_{i S F}$ vohergesagt werden, welche Versetzungsnukleation am wahrscheinlichsten erscheint. Die twinning-Tendenz $m_{T}$ ist nach Li et al. [17] demnach gegeben durch das Verhältnis

$$
m_{T}=\frac{\gamma_{u S F}-\gamma_{i S F}}{\gamma_{u T}-\gamma_{i S F}}
$$

Sollen die aufgelösten Schubspannungen, wie sie im vorherigen Abschnitt beschrieben sind, mit dem Bild der generalisierten Stapelfehler verbunden werden, ist es erforderlich, die Energiebarrieren für die einzelnen Prozesse mit den jeweiligen Schmidfaktoren zu gewichten[19]. Die kritische Spannung für die Nukleation der verschiedenen Partialversetzung kann dann wie folgt abgeschätzt werden: Für eine weitere leadingPartialversetzung auf einer nicht angrenzenden Ebene durch $\sigma_{p}^{c} \propto \gamma_{u S F} / m_{\text {lead }}$, für eine trailing -Partialversetzung auf der gleichen Ebene durch $\sigma_{f}^{c} \propto\left(\gamma_{u S F}-\gamma_{i S F}\right) / m_{\text {trail }}$ sowie für eine twinning-Partialversetzung auf einer benachbarten Ebene durch $\sigma_{t}^{c} \propto$ $\left.\left(\gamma_{u T}-\gamma_{i S F}\right) / m_{\text {lead }}\right)$. Damit lässt sich eine Verformungskarte, wie sie in Abbildung 2.4 (b) dargestellt ist, aus zwei Paramtern aufspannen[19]:

$$
\tau_{1}=\frac{\sigma_{p}^{c}}{\sigma_{t}^{c}}=\frac{\gamma_{u S F}}{\gamma_{u T}-\gamma_{i S F}}
$$

gibt an, ob eine twinning- oder eine neue leading-Partialversetzung bevorzugt ist.

$$
\tau_{2}=\frac{\gamma_{u S F}-\gamma_{i S F}}{\gamma_{u T}-\gamma_{i S F}} \cdot \frac{m_{\text {lead }}}{m_{\text {trail }}}
$$

gibt an, ob eine twinning- oder eine trailing-Partialversetzung und damit eine Verformung über volle Versetzungen bevorzugt ist. Dabei ist zu beachten, dass in $\tau_{2}$ der twinning-Faktor $m_{T}$ aus [17] auftaucht und somit auch in diesem Bild vorhanden ist. Die Verformungskarte lässt sich nun in drei Bereiche teilen: Verformung durch vereinzelte Partialversetzungen $\left(\tau_{1}<\tau_{2}\right.$ und $\left.\tau_{1}<1\right)$, Verformung durch volle Versetzungen $\left(\tau_{2}<\tau_{1}\right.$ und $\left.\tau_{2}<1\right)$ sowie einer Verformung durch twinning $\left(\tau_{1}>1\right.$ und $\left.\tau_{2}>1\right)$. Für einen Zugversuch von Gold in die $\langle 110\rangle$-Richtung ist die Position in der Karte eingezeichnet. Demnach lässt sich entsprechend diesem Bild eine Verformung durch Zwillingsbildung erwarten. 


\subsubsection{Oberflächengeometrie}

Im Gegensatz zu Bulk-Materialien weisen Nanodrähte ein hohes Maß an Oberfläche im Vergleich zu ihrem Volumen auf. Zahlreiche MD-Simulationen haben gezeigt, dass die Querschnittsform und damit die Orientierung der Oberflächen eine entscheidende Rolle für die Verformung spielen kann [18][27][19].

Um die Wirkung der Oberflächen zu berücksichtigen, kann man grundsätzlich die Energien einer Oberflächenstufe betrachten. Die Nukleation einer Partialversetzung von einer glatten Oberfläche führt zur Bildung einer Oberflächenstufe, die die Energiebarriere für die Nukleation erhöht. Die Nukleation einer twinning-Partialversetzung kann dagegen zu einer Senkung der Stufenenergie führen, indem die Oberfläche zu einer anderen niedrig indizierten Oberfläche mit einer niedrigen Oberflächenenergie reorientiert wird.
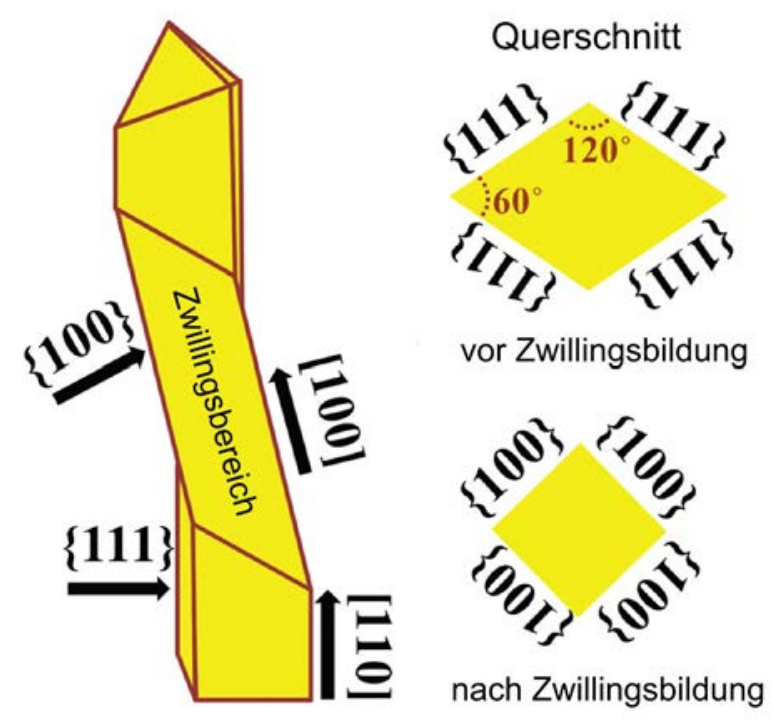

Abbildung 2.5.: Schematische Darstellung zur Rerorientierung eines Drahtes durch Zwillingsbildung (aus [28])

Bei fcc-Metallen weisen die $\{111\}$-Facetten die kleinsten Oberflächenenergien auf, gefolgt von den $\{100\}$-Facetten und den $\{110\}$-Facetten. Bei $\langle 110\rangle$-Drähten, die lediglich $\{111\}$-Facetten aufweisen, können sich durch Verformung Zwillinge mit sauberen $\{100\}$-Facetten bilden (siehe Abbildung 2.5), was eine Zwillingsbildung ermöglicht [18]. Der Querschnitt von Drähten, wie sie häufig experimentell verwendet werden, kann neben den vier dominanten $\{111\}$-Facetten zwei mehr oder weniger ausgeprägte $\{100\}$ Facetten aufweisen, wie es auch gemäß einer Wulff-Konstruktion für $\langle 110\rangle$-Drähte er- 
wartet wird. MD-Simulationen haben gezeigt, dass je nachdem wie stark diese beiden $\{100\}$-Facetten ausgeprägt sind, es zu einem Wechsel im Verformungsmechanismus kommen kann [29][30]. Drähte mit stark ausgeprägten $\{100\}$-Facetten tendieren zu einer Verformung durch separierte Partialversetzungen, Drähte mit weniger stark ausgeprägten $\{100\}$-Facetten tendieren zur Verformung durch twinning-Partialversetzungen.

\subsection{Klassische Keimbildung}

Eine Versetzungsnukleation lässt sich auch im Kontext der klassischen Keimbildung verstehen. Die Keimbildungsrate und entsprechend die Nukleationsrate für Versetzungen ist in der klassischen Keimbildungstheorie bei einer Temperatur $T$ und einer angelegten Spannung $\sigma$ gegeben durch [31][16][10]:

$$
I(\sigma, T)=\nu_{0} n_{0} \exp \left(\frac{E_{c}(\sigma, T)}{k_{b} T}\right)
$$

$\nu_{0}$ ist die Anklopffrequenz und ein Maß für die Wahrscheinlichkeit, mit der ein Atom dem Keim beitreten kann. Nach Hirth und Lothe[16] ergibt sich dieser Vorfaktor durch $\nu_{0}=8 \pi R_{c} \nu / b$, wobei $R_{c}$ der kritische Keimradius und $\nu$ die Debyefrequenz ist. Oft wird der Vorfaktor jedoch direkt mit der Debye-Frequenz genähert $\left(\nu_{0} \approx \nu\right)[19] . n_{0}$ ist die Anzahl von äquivalenten Nukleationplätzen. Die Energiebarriere $E_{c}(\sigma, T)$ kann aus atomistischen Simulationen durch die Anwendung von 'Chain-of-states'-Methoden wie 'Nudged-Elastic-Band'-Kalkulationen [32] bestimmt werden [10][27]. Ebenso kann $E_{c}(\sigma, T)$ aus einem elastischen Kontinuumsmodell ermittelt werden, indem die Änderung der Energie eines isotropen Kristalls durch die Einführung einer Versetzung betrachtet wird, was zuerst von Hirth und Lothe [16] eingeführt wurde und was als Grundprinzip in vielen weiteren Studien zur Beschreibung der Versetzungsnukleation verwendet wurde [33][34][27][35][36]. Nach Hirel et al. [34] sind in diesem Modell für die Nukleation eines Versetzungshalbrings mit dem Radius R insgesamt vier Energieterme zu berücksichtigen:

$$
E=E_{d}+E_{\text {relax }}+E_{s}+E_{\gamma}
$$


$E_{d}$ stellt die Linienenergie der Versetzung dar und ergibt sich nach [34] aus

$$
E_{d}=\frac{\mu b^{2}(2-\nu)}{8(1-\nu)} R\left[\ln \left(\frac{8 m \alpha R}{b}\right)-2\right]
$$

Hierbei ist $\mu$ das Schermodul, $\nu$ der Poissionkoeffizient, $\alpha$ ein Faktor durch den unbekannten Kernradius der Versetzung und $m$ ein Korrekturfaktor für die Bild-Kräfte der Oberfläche [37], der einen großen Einfluss auf das Gesamtverhalten hat. $E_{\text {relax }}$ ist die Arbeit, die durch die Spannungsrelaxation bei der Vergrößerung des Versetzungsrings gewonnen wird. Sie ist proportional zur Fläche, die von der Versetzung überstrichen wurde, und ergibt damit für einen Versetzungshalbring

$$
E_{\text {relax }}=-1 / 2 \pi R^{2} b \tau=-1 / 2 \pi R^{2} b \sigma m_{s}
$$

wobei b der Burgersvektor der Versetzung ist und $\tau$ die Scherspannung auf die Versetzung, die sich mit Hilfe des Schmidfaktors $m_{s}$ aus der angelegten Spannung $\sigma$ bestimmen lässt. $E_{s}$ ist die Energie, die durch die Ausbildung bzw. Rückbildung einer Oberflächenstufe aufgebracht bzw. gewonnen wird. Sie ergibt sich aus

$$
E_{s}= \pm 2 R \cos (\Theta) b \sigma_{s}
$$

wobei $\Theta$ der Winkel zwischen Burgersvektor und Lage der Oberflächenstufe ist und $\sigma_{s}$ die Energie der Oberflächenstufe. $E_{\gamma}$ ist die Energie, die im Fall einer Partialversetzung durch die Erzeugung eines Stapelfehlers aufgebracht werden muss. Sie bestimmt sich unter der Berücksichtigung der Stapelfehlernergie $\gamma$ wie folgt:

$$
E_{\gamma}=1 / 2 \pi R^{2} \gamma
$$

Der kritische Keimradius $R_{C}$ und die Energiebarrie $E_{c}$ lassen sich nun in Abhängigkeit der von außen angelegten Spannung durch Maximierung von Gleichung 2.7 $\left(d E /\left.d R\right|_{R_{C}}=0\right.$ und $\left.d^{2} E /\left.d R^{2}\right|_{R_{C}}<0\right)$ ermitteln.

Roos [11] hat in seinem Modell die klassische Nukleationsrate (Formel 2.6) mit den Ergebnissen für die Energiebarriere $E_{C}(\sigma)$ und die kritischen Keimradien $R_{c}(\sigma)$ aus dem elastischen Modell für einen Versetzungshalbring zusammengeführt. Mit einer geeigneten Wahl der Anzahl an Nukleationsplätzen für die verschiedenen Partialversetzungen erhält man die Nukleationsraten für die jeweilige Partialversetzung in 
Abhängigkeit der äußeren Spannung wie in Abbildung 2.6 zu sehen ist. ${ }^{1}$

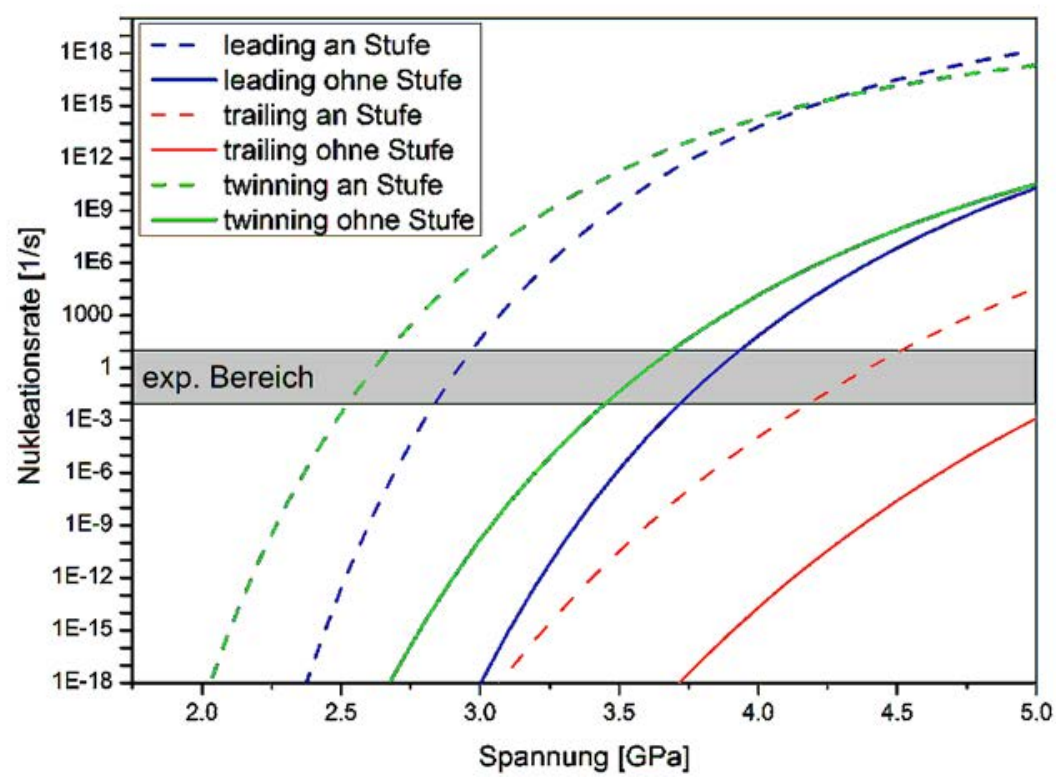

Abbildung 2.6.: Nukleationsraten für verschiedene Partialversetzungen nach dem Modell von Roos[11] mit Abbau einer Oberflächenstufe (gestrichelte Kurven) oder Entstehung einer Stufe (durchgezogenen Kurven)

In dem experimentell erfassbaren Bereich, bei dem man von Raten zwischen einem Event alle 100 Sekunden bis zu 10 Events pro Sekunde ausgegangen ist, benötigt die Nukleation der twinning-Partialversetzung an einer Stufe die niedrigste von außen angelegte Spannung. Sofern also Oberflächenstufen in Kombination mit einem vorhandenen planaren Defekt vorhanden sind, ist die Nukleation von twinning-Partialversetzungen zu erwarten. Ist diese Kombination nicht vorhanden, wird als nächstes die Nukleationsspannung für eine neue leading-Partialversetzung an einer Stufe erreicht. Sollten keine Stufen mehr vorhanden sein, wäre erneut die Nukleationsspannung für die twinning-Partialversetzung am niedrigsten. Die Nukleationsspannungen für die trailingPartialversetzungen liegen sowohl mit als auch ohne Stufe deutlich höher, so dass keine Nukleation von trailing-Partialversetzungen zu erwarten ist.

\footnotetext{
${ }^{1}$ Roos hat in seiner Arbeit für $E_{\text {relax }}$ statt Formel 2.8 einen Ausdruck von Hirel et al. [34] verwendet. Wie mit den Autoren von [34] kommuniziert, hat sich dieser Ausdruck als fehlerhaft herausgestellt [38]. Abbildung 2.6 zeigt demnach eine korrrigierte Version des Modells von Roos. Aussagen über Mechanismen haben sich nicht geändert. Allerdings führen die angepassten Rechnungen zu wesentlich veränderten Spannungswerten im Vergleich zu der ursprünglichen Version von Roos.
} 


\subsection{Fließgrenze bei Versetzungsnukleation}

In allen existierenden MD Simulationen ist die Nukleation der leadingPartialversetzung das erste plastische Event und damit der limitierende Schritt, der die Fließgrenze bestimmt. Da es sich bei der Versetzungsnukleation um einen thermisch aktivierten Prozess handelt, ist es nicht möglich, einen exakten Wert als Fließgrenze bzw. Nukleationsspannung anzugeben. Stattdessen wird eine Spannung angegeben, bei der die Nukleation am wahrscheinlichsten stattfindet.

Nach dem Modell von Roos [11] ist die Fließgrenze gerade bei der Spannung erreicht, bei der die Nukleationrate in den experimentell beobachtbaren Bereich eintritt. Für eine leading-Partialversetzung an einer Stufe ist dies bei einer Spannung von $\sigma_{c, \text { Roos }}=2,85 G P a$ der Fall.

Andere Studien [39][10][27] haben einen mathematischen Ausdruck für die wahrscheinlichste Nukleationsspannung aus der klassischen Nukleationstheorie mit Gleichung 2.6 abgeleitet. Diese Gleichung ist jedoch nur gültig bei einer konstanten Spannung, wohingegen Experimente gewöhnlich unter einer konstanten Dehnrate durchgeführt werden. Um diesen Umstand zu berücksichtigen haben verschiedene Autoren unter der Annahme einer konstanten Dehnrate $(\sigma=\dot{\epsilon} E t)$ einen impliziten Ausdruck für die wahrscheinlichste Nukleationsspannung berechnet [39][10][27]:

$$
\frac{E_{c}(\sigma, T)}{k_{b} T}=\ln \left[\frac{k_{b} T n_{0} \nu_{0}}{E \dot{\epsilon} \Omega(\sigma, T)}\right]
$$

$\Omega(\sigma, T)=\left.\frac{-\partial E_{c}}{\partial \sigma}\right|_{T}$ ist das Aktivierungsvolumen und gibt die Sensitivität der Nukleationsrate von der Spannung wieder. Mit der Kenntnis von $E_{c}$ und einer sinnvollen Abschätzung für die Anzahl an Nukleationsplätzen $n_{0}$ kann die wahrscheinlichste Fließspannung $\sigma_{y}$ durch numerisches Lösen von Gleichung 2.9 berechnet werden. Es bleibt zu beachten, dass für die o.a. Gleichung ein linear elastisches Verhalten angenommen wird. Wie beispielsweise in [40] oder [41] und auch in den Ergebnissen dieser Arbeit zu sehen ist (siehe Abschnitt 4.4.3), gilt das linear elastische Verhalten bei Nanodrähten für hohe Dehnungen nicht mehr, so dass die Nukleationsspannung mit der o.a. Gleichung überschätzt wird.

Weinberger et al. [27] konnten durch Lösen von Gleichung 2.9 bei einer Temperatur von $300 \mathrm{~K}$ und einer Dehnrate von $\dot{\epsilon}=10^{-3} / \mathrm{s}$ sowie aus atomistischen Simulationen ermittelten Energiebarrien $E_{c}$ die wahrscheinlichste Nukleationsspannung bei einem 
Zugversuch für einen $\langle 110\rangle$-Golddraht zu $\sigma_{c, \text { Sim }}=1,5 \mathrm{GPa}$ berechnen. Verwendet man dagegen die Energiebarrieren $E_{c}$ aus dem elastischen Kontinuumsmodell für die Nukleation eines Halbrings, so erhält man bei gleichen Parametern für Temperatur und Dehnrate als wahrscheinlichste Nukleationsspannung $\sigma_{c, \text { Halbring }}=2,95 G$ Pa. Dieses Ergebnis liegt nahe an der einfachen Abschätzung aus dem Modell von Roos, ist aber fast doppelt so hoch wie die berechnete Nukleationsspannung aus den atomistischen Simulationen.

Um eine bessere Übereinstimmung sowohl zu Simulationen als auch hinsichtlich Experimenten zu erhalten, wurde das elastische Kontinuumsmodell von Jennings et al. [35] speziell zur Vorhersage von Nukleationsspannungen von Nanodrähten unter experimentellen Bedingungen angepasst. So haben sie statt der Nukleation eines Halbrings die Nukleation an einer $120^{\circ}$-Ecke betrachtet, wie es die Simulationen ebenfalls zeigen [27]. Zusätzlich haben sie angepasste Werte für den Burgersvektor sowie für die Stapelfehlerenergie nach dem Modell von Aubry et el. [42] verwendet. Ebenfalls wurde die Temperaturabhängigkeit der Energie und damit der Einfluss der Entropie durch einen Vorfaktor von $\left(1-T / T^{*}\right)$ berücksichtigt, wobei $T^{*}$ die Hälfte der Schmelztemperatur ist [10]. Mit diesen Veränderungen im elastischen Modell konnten Jennings et al. [35] die Nukleationspannung zu $\sigma_{c, \text { Jennings }}=1,22 G P a$ ohne die Berücksichtigung der Erzeugung einer Oberflächenstufe bzw. $\sigma_{c, \text { Jennings }_{2}}=1,52 G P a$ mit Berücksichtigung der Erzeugung einer Oberflächenstufe bestimmen. Dies ist nahezu identisch mit den berechneten Werten aus den atomistischen Simulationen.

\begin{tabular}{c|c} 
& wahrscheinlichste \\
& Nukleationsspannung [GPa] \\
\hline Halbring - Modell Roos [11] & 2,85 \\
Halbring - nach Gleichung 2.9 & 2,95 \\
Draht - Simulation [27] & 1,5 \\
Draht - nach Jennings [35] & 1,22 bzw. 1,52 \\
\hline
\end{tabular}

Tabelle 2.1.: Wahrscheinlichste Nukleationsspannung aus verschiedenen Modellen

\subsection{Größeneffekte bei der Versetzungsnukleation}

Mechanische Eigenschaften werden häufig in Verbindung gebracht mit einem Größeneffekt sowohl für den Verformungsmechanismus als auch für die Festigkeit. Wie im 
vorherigen Abschnitt gezeigt, ist auf den ersten Blick kein Größeneffekt für die Nukleationsspannung aus Gleichung 2.9 zu erkennen. Durch eine lineare Entwicklung der Energiebarriere in Hinblick auf die Spannung gelangt man zu einem angenäherten Ausdruck für die Festigkeit [27]:

$$
\sigma_{c}=\frac{E_{0}}{\Omega_{0}}-\frac{k_{b} T}{\Omega_{0}} \ln \left(\frac{k_{b} T n_{0} \nu_{0}}{E \dot{\epsilon} \Omega_{0}}\right)
$$

Gemäß dieser Gleichung ist für die Nukleatiosspannung lediglich ein unbedeutender Größeneffekt über den logarithmischen Zusammenhang mit der Anzahl an Nukleationspunkten $\ln \left(n_{0}\right)$ zu erwarten, der lediglich einen zu vernachlässigenden Anstieg der Festigkeit für kleinere Proben erwarten lässt.

Bisher wurde der Einfluss von Oberflächenspannungen außer Acht gelassen. Die Oberflächenatome erfahren aufgrund der geringeren Anzahl an nächsten Nachbarn eine Zugspannung. Um diesen Oberflächeneffekt auszugleichen, entsteht eine Kompressionsspannung entlang der Drahtachse. Die Kompressionsspannung ist näherungsweise durch $\sigma_{\text {axis }} \approx 4 f / D$ gegeben, wobei sich $f$ in der Größenordnung der Oberflächenenergie bewegt und $D$ der Durchmesser des Drahtes ist [19][27]. Für Zugversuche wird demnach eine Zunahme der Festigkeit für Drähte mit geringerem Durchmesser erwartet. Dieser Effekt ist hauptsächlich bei Drähten mit einem Durchmesser zwischen 5nm und 40nm sichtbar und kann für Proben mit größerem Durchmesser vernachlässigt werden [27].

Neben dem vernachlässigbaren Größeneffekt auf die Festigkeit bei der Verformung über Versetzungsnukleation haben die bisher vorgestellen Modelle ebenfalls keinen Größeneffekt auf die Art der Verformung gezeigt. Es existieren jedoch weitere Modelle in der Literatur, die die beobachtete Verformung (z.B. das Auftreten von Zwillingen) als einen größenabhängigen Wechsel im Verformungsmechanimus interpretieren.

Im ersten dieser Modelle wird die Aufspaltungsweite von Partialversetzungen für einen Übergang zwischen vollen Versetzungen in makroskopischen Proben und Partialversetzungen in nanoskaligen Proben als Erklärung verwendet. Der Übergang findet genau dann statt, wenn die Aufspaltungsweite größer als die Dimension der Probe wird. Nach Byun [43] ist die Aufspaltungsweite von Partialversetzungen abhängig von der außen angelegten Spannung. Ab einer bestimmten Spannung würde dann die Aufspaltungsweite die Dimension der Probe übertreffen und somit zu einem Wechsel im Verfor- 
mungsmechanismus führen [44]. Den Übergang für Gold-Drähte würde man hiernach jedoch erst unter einem Durchmesser von 6nm erwarten [11]. Alle bisherigen Ergebnisse zeigen jedoch schon bei wesentlich größeren Proben von $\mathrm{d}>150 \mathrm{~nm}$ eine Verformung über Partialversetzungen [11][20][21]. Weiterhin haben van Swygenhoven et al. [45] herausgestellt, dass die Aufspaltungsweite auf diese Weise nur für zwei existierende Partialversetzungen gültig ist. Im Fall einer Versetzungsnukleation muss man jedoch zwischen der Nukleation selbst und der Bewegung der Versetzung trennen, wobei die Nukleation im Allgemeinen den limitierenden Faktor darstellt.

In einem weiteren Modell vergleicht man die verschiedenen Spannungen, welche erforderlich sind, eine Frank-Read-Quelle für die Nukleation einer vollen Versetzung bzw. einer Partialversetzung zu aktivieren. Für die Nukleation einer vollen Versetzung wird demnach eine Scherspannung von $\tau_{N}=2 \alpha \mu b_{N} / D$ benötigt und für die Nukleation einer Partialversetzung eine Scherspannung entsprechend $\tau_{p}=2 \alpha \mu b_{P} / D+\gamma / b_{P}[16]$. $b_{N}$ und $b_{P}$ sind hierbei die Burgersvektoren einer vollen Versetzung bzw. einer Partialversetzung, $\alpha$ beschreibt den Charakter der Versetzung (0,5 für eine Stufenversetzung, 1,5 für eine Schraubenversetzung), $\gamma$ bezeichnet die Stapelfehlerenergie, $\mu$ das Schermodul und D die Größe der Quelle. Chen et al. [46] assoziierten die Größe der Quelle mit der Korngröße in nanokristallinem Aluminium. Wenn die Korngröße unter einen kritischen Wert

$$
D_{c}=\frac{2 \alpha \mu\left(b_{N}-b_{P}\right) b_{P}}{\gamma}
$$

fällt, wird die Nukleationsspannung für eine Partialveretzung $\tau_{p}$ kleiner als für eine volle Versetzung $\tau_{N}$ und man würde einen Wechsel im Verformungsmechanismus erwarten. Oh et al.[47] haben diesen Ansatz auf die Verformung von dünnen Goldschichten angewendet und so eine kritische Schichtdicke von circa $16 \mathrm{~nm}$ bestimmt. Sedlmayr et al. [21] haben dieses Kriterium schließlich um die Schmidfaktoren für die jeweiligen Versetzungen erweitert. Angewandt auf Golddrähte mit einer Zuggeometrie in $\langle 110\rangle$ Richtung führt dies zu einem Übergangswert von $D_{C}=40 \mathrm{~nm}$. Zu ähnlichen Werten kommen Hwang et al. [28], ebenfalls für $\langle 110\rangle$-Golddrähte. 


\section{Experimentelle Methoden}

In diesem Abschnitt werden die verwendeten experimentellen Methoden beschrieben. Hauptaugenmerk liegt hierbei auf den beiden Methoden für die Zugversuche im TEM sowie im REM. Dazu wird deren Aufbau sowie der Prozess zur Probenpräparation vorgestellt und danach auf den Ablauf eines Zugversuchs eingegangen. Abschließend werden die Methoden erläutert, die dazu verwendet wurden, vor dem Zugversuch gezielt Defekte in ausgewählten Drähten zu erzeugen.

\subsection{Verwendete Geräte}

In diesem Abschnitt erfolgt eine kurze Beschreibung der für die Tests eingesetzten Geräte. Hierzu gehören das Rasterelektronenmikroskop, das fokussierte Ionenstrahlmikroskop und das Transmissionselektronenmikroskop.

\subsubsection{Rasterelektronenmikroskop (REM)}

Das Rasterelektronenmikroskop wurde in dieser Arbeit sowohl für die Probenpräperation als auch für die quantitaiven Zugversuche verwendet. Im Zuge dieser Arbeit kamen dabei ein REM vom Typ FEI Nova NanoSEM 650 sowie ein Dual-Beam-System (Kombination aus REM und Ionenstrahlmikropskop) vom Typ FEI Nova NanoLab 600 zum Einsatz.

Im REM wird ein Elektronenstrahl mit einer Spannung von bis zu 30kV beschleunigt, anschließend fokussiert und über die Probe gerastert. Dabei kommt es zur Wechselwirkung zwischen den Elektronen und dem Probenmaterial, wodurch beispielsweise niederenergetische Sekundärelektronen entstehen, die hauptsächlich aus der Oberfläche der Probe stammen und somit zum Abbilden der Topographie verwendet werden können. Die Zahl der Sekundärelektronen kann pro Rasterpunkt mit einem Detektor bestimmt und anschließend in einen Bildkontrast übersetzt werden. Zusätzlich ist in 
beiden System ein Gas-Injektions-Systemen für die Abscheidung von Platin installiert sowie der Einsatz von Mikromanipulatoren (Firmen Kleindiek oder Omniprobe) möglich [48].

\subsubsection{Ionenstrahlmikropskop (FIB)}

Das Ionenstrahlmikropskop fand im Rahmen dieser Arbeit für die Herstellung von Probenquerschnitten für die Analyse der Querschnittsfläche der Drähte sowie die Präparation von Querschnitts-Lamellen für weitergehende Untersuchungen mit dem Tranmissionelektronenmikropskop Anwendung. Im FIB werden Ionen, typischerweise Galliumionen, analog zum REM mit einer Spannung von bis zu $30 \mathrm{kV}$ beschleunigt, fokussiert und anschließend über die Probe gerastert. Dabei entstehen ebenfalls Sekundärelektronen, die sich zum Abbilden der Oberfläche nutzen lassen. Im Gegensatz zu den Elektronen im REM ist die kinetische Energie der Ionen ungleich größer. Somit kommt es zu einem Abtrag von Material aus der Oberfläche. Durch Vorgabe des Rastergebiets kann so gezielt Material abgetragen und die Oberfläche strukturiert werden.

\subsubsection{Transmissionselektronenmikropskop (TEM)}

Bei der Transmissionselektronenmikroskopie werden die Proben mit hochenergetischen Elektronen durchstrahlt. Die Probendicke ist deshalb je nach Material auf wenige 100nm beschränkt. Die Elektronen werden mit Spannungen zwischen 80kV und 300KV beschleunigt und anschließend durch mehrere Kondensorlinsen auf die Probe fokussiert. Die Wechselwirkung mit der Probe erfolgt hauptsächlich durch elastische Streuung am Kristallgitter. Hinter der Probe befindet sich das Objektivlinsensystem, das ein Zwischenbild der durchstrahlten Probe liefert. Dieses Zwischenbild kann durch ein System aus Projektivlinsen mehrstufig vergrößert und auf einem Fluoreszenzschirm oder mit einer CCD-Kamera sichtbar gemacht werden. Neben einer Vergrößerung des Realbildes lässt sich das Abbildungssystem auch so steuern, dass die hintere Brennebene des Objektivs vergrößert dargestellt wird und man so ein Beugungsbild der Kristallstruktur erhält. Zur Bildgebung kann zusätzlich eine Blende in der Beugungsebene in den Strahlengang eingebracht werden. Diese Blende ist auf der einen Seite so justierbar, dass nur die ungebeugten Elektronen zur Bildgebung beitragen (Hellfeld). Auf der anderen Seite besteht die Möglichkeit, die Blende so zu justieren, dass die Elektronen zur Bildgebung 
beitragen, die an einem bestimmten Ebenentyp gebeugt wurden (Dunkelfeld).

\subsection{Zugapparaturen}

\subsubsection{TEM-Aufbau}

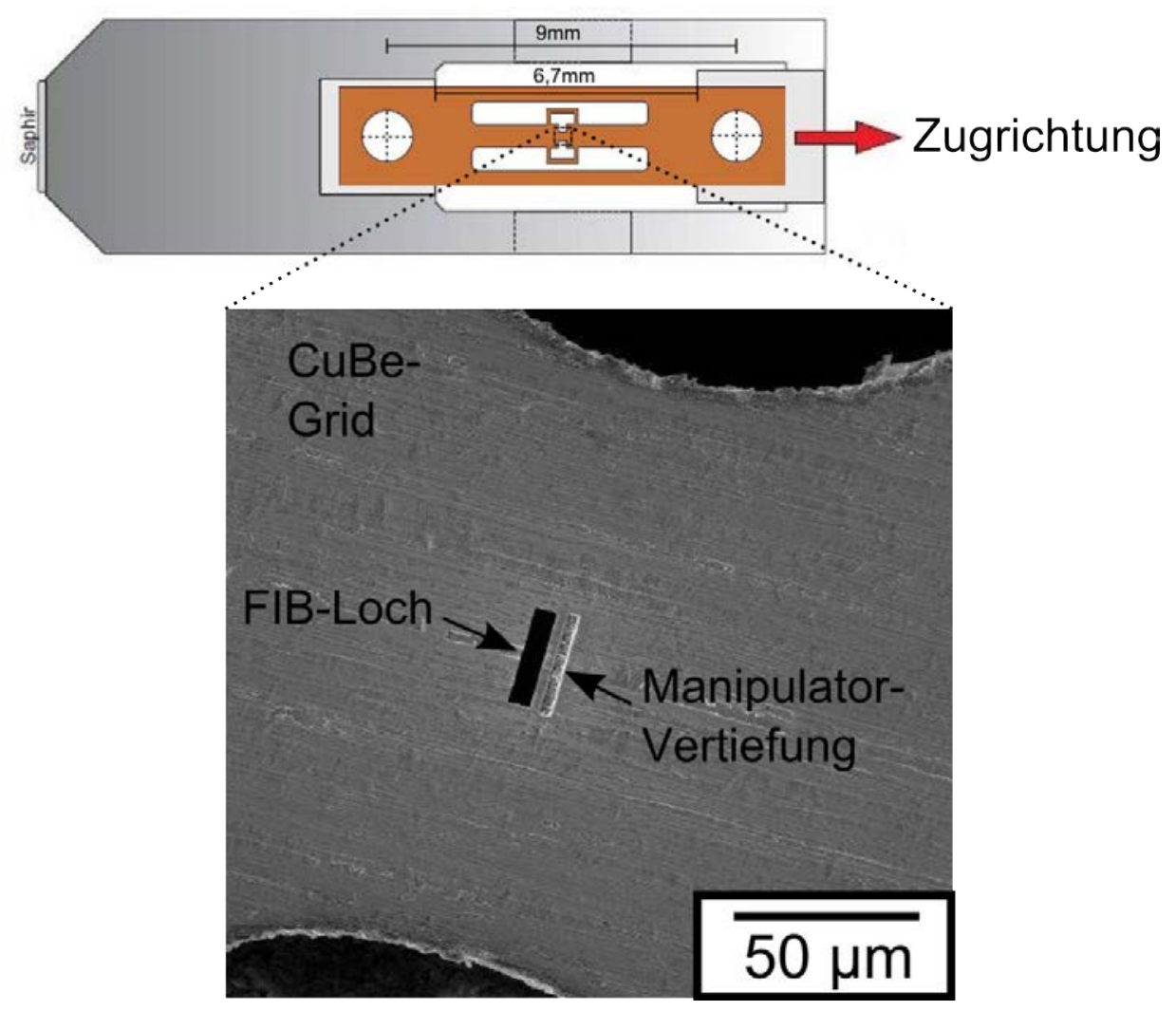

Abbildung 3.1.: Aufbau im TEM: Der obere Teil zeigt schematisch die Spitze des TEMHalters mit dem CuBe-Grid (orange); der untere Teil zeigt eine REMAufnahme des mittleren Teils des CuBe-Grids.

Für die Zugversuche im TEM kommt ein Zughalter der Firma Gatan vom Modell 654 zur Anwendung, bei dem es sich um einen Einfach-Kipp-Halter handelt. Eine Darstellung der Spitze des Zughalters ist in Abbildung $3.1 \mathrm{zu}$ sehen. Die beiden Aufnahmepunkte für eine Probe liegen 9mm auseinander. Einer der Aufnahmepunkte (in Abbildung 3.1 der rechte) ermöglicht eine Bewegung mit einer minimalen Schrittweite von circa $5 \mathrm{~nm}[11]$.

Passend zur Aufnahme des Zughalters wird eine vorstrukturierte KupferBeryllium(CuBe)-Folie verwendet, wie sie in Abbildung 3.1 in orange zu sehen ist. 
Diese wird mit Hilfe von zwei Unterlegscheiben und zwei Schrauben in den beiden Aufnahmepunkten befestigt. In der Mitte CuBe-Folie wird mit Hilfe des FIBs zum einen

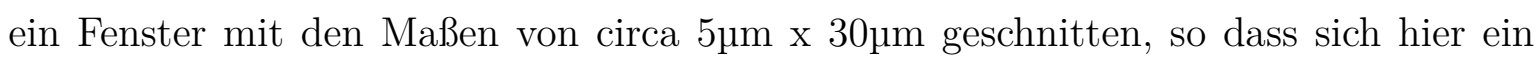
elektronentransparenter Bereich für das TEM ergibt. Zum anderen wird neben dieses Fenster ein kleiner Graben geschnitten, so dass die Manipulatorspitze während des Transferprozesses leicht unter die Oberfläche eintauchen kann. Eine REM-Aufnahme des präparierten CuBe-Grids ist in Abbildung 3.1 unten zu sehen. Die CuBe-Folie sorgt während des Zugversuchs dafür, dass sich die Verformungsschritte des Zughalters homogen zwischen den beiden Aufnahmepunkten (bzw. dem 6,7mm breiten Bereich zwischen den beiden Unterlegscheiben) verteilen. In einer einfachen Abschätzung würden sich auf das 5um breite Fenster Dehnungschritte entsprechend einer Größe von

$$
\Delta l=\epsilon \cdot l_{0}=\frac{5 n m}{6,7 m m} \cdot 5 \mu m \approx 0,004 n m
$$

ergeben. Finite Element Simulationen haben allerdings gezeigt, dass sich unter Berücksichtigung der Geometrie der CuBe-Folie die Dehnungsschritte des Zughalters um einen Faktor 3 und damit auf 1,67nm große Dehnungsschritte auf das Fenster verkleinern lassen. Eine eindeutige Bestimmung der Dehnungsschritte erweist sich also als schwierig. Jedoch kann davon ausgegangen werden, dass ein Dehnungsschritt im Bereich von unter einem Burgersvektor einer typischen Versetzung bis zu circa zehn Burgersvektoren liegt.

\subsubsection{REM Aufbau}

Der nanomechanische Zugaufbau, der im Rahmen meiner Diplomarbeit entstanden und im Zuge dieser Arbeit weiterentwickelt wurde, besteht aus einem Kraftsensor und drei Aktuatoren, die alle vakuumkompatibel sowie klein und leicht sind, so dass sie auf den Probentisch eines REMs montiert werden können. Der Kraftsensor ist eine kommerziell erhältliche Platine bestehend aus einem mikro-elektro-mechanischem System (MEMS) und integrierter Elektronik (Femtotools). Die messbare Kraftreichweite beträgt $\pm 100 \mu N$ mit einer nominellen Auflösung von $\pm 5 n N$. Eine externe Kraft, die parallel zur Sensorachse angelegt wird, führt zu kleinen Abstandsänderungen in einem federgeladenen Kamm-Kondensator, was zu einem Spannungssignal führt, das proportional zur außen angelegten Kraft ist [49]. Jeder Sensor ist individuell vom Hersteller 


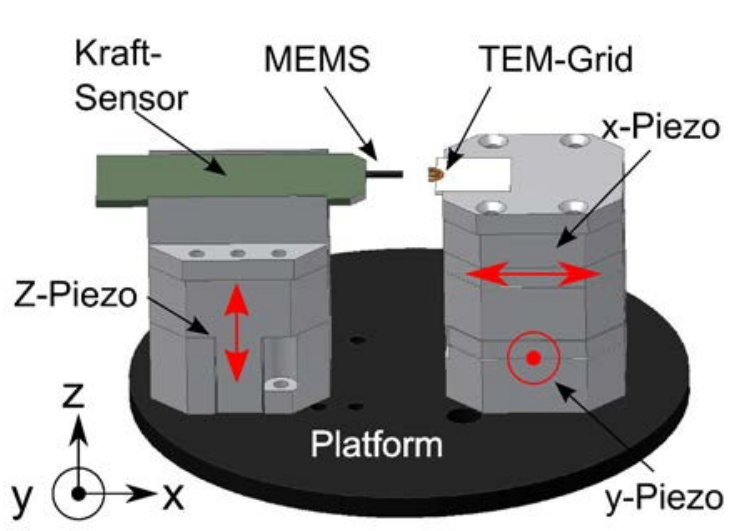

(a)

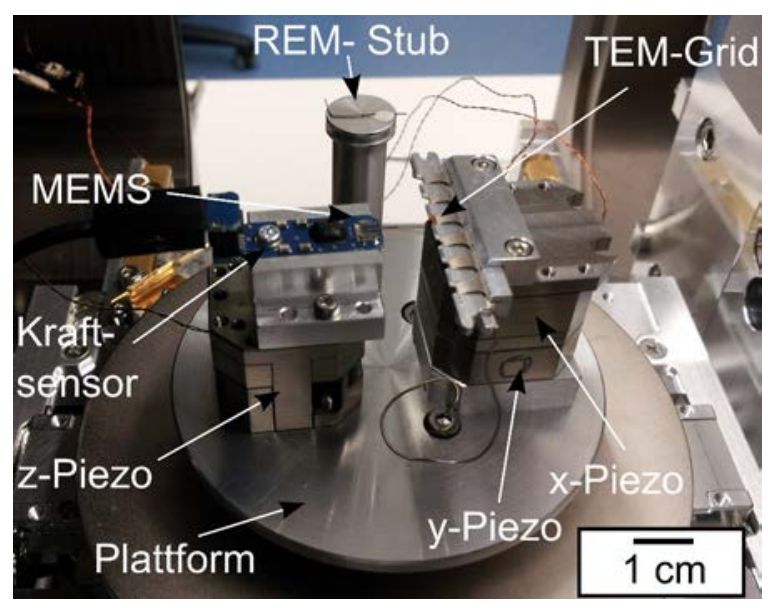

(b)

Abbildung 3.2.: Schematische Zeichnung (a) und Foto (b) des REM-Aufbaus

kalibriert mit typischen Werten um $50 \mu N / V$. Bei den drei Aktuatoren für jeweils eine Raumrichtung handelt es sich um open-loop kontrollierte, piezoelektrisch basierte Aktuatoren. Mit dem slip-stick-Prinzip sind zum einen Bewegungen mit Schrittgrößen von unter 1nm zum anderen ein großer Verfahrweg von bis zu $5 \mu \mathrm{m}$ möglich.

Alle Komponenten sind auf einer Plattform mit einem Durchmesser von circa $7 \mathrm{~cm}$ montiert, die entweder in ein REM oder FIB eingebaut werden kann. Der x- und der y-Positionierer sind übereinander auf einer Seite der Plattform angebracht sowie ein TEM-Halbmondgrid, das oben auf den Positionierern befestigt und mit einem Kupferdraht mit der Plattform verbunden ist, um dadurch Aufladungseffekte zu vermeiden. Der TEM-Halbmond kann nach dem Versuch einfach in einen Doppelkipphalter für das TEM eingebaut werden um post-mortem Untersuchungen der getesteten Proben zu ermöglichen. Der z-Positionierer, auf dem der Kraftsensor montiert ist, befindet sich auf der gegenüberliegenden Seite der Plattform. Der Sensor ist so ausgerichtet, dass die Bewegung in x-Richtung genau parallel zur Messachse des Sensors erfolgt. Eine Dehnung der Probe erfolgt durch den x-Positionierer und die resultierende uniaxiale Kraft wird mit dem Sensor gemessen. Auf der Plattform befindet sich weiterhin ein Montierplatz für das Probenmaterial, so dass sich die Proben auf die Testapparatur bringen lassen, ohne die Kammer öffnen zu müssen. Bis jetzt wurde das System in einem REM vom Typ FEI Nova NanoSEM 650 sowie einem Dual-Beam-System vom Typ FEI Nova NanoLab 600 erfolgreich verwendet. Beide Systeme verfügen dabei über einen Probentisch, der Bewegung in drei Raumrichtungen sowie Rotation und Kippen 
zulässt. Die Verkabelung, die für die Positionierer sowie den Kraftsensor erforderlich ist, werden mit zwei vakuumkompatiblen sub-D15 Durchführungen realisiert, die in einen Flansch für die jeweilige Vakuumkammer geschweißt sind.

\subsection{Proben, Transfer und Ablauf der Versuche}

Für die Zugversuche wurden Nanodrähte aus Gold (Silber und Kupfer, siehe C) verwendet. Diese wurden via Molecular Beam Epitaxy bei erhöhten Temperaturen auf Silizium oder Wolfram Substraten erzeugt [50][51][52]. Die entstandenen Drähte sind überwiegend einkristallin; können aber auch eine oder mehrere kohärente $\Sigma 3$-Zwillingsgrenzen aufweisen. Dabei sind die Drähte größtenteils defektfrei mit einer Länge von bis zu circa $50 \mu m$. Die Achse verläuft für alle Drähte entlang einer $\langle 110\rangle$-Richtung. Der Querschnitt der Drähte ist stark facettiert. Bei den Seitenflächen handelt es sich hauptsächlich um $\{111\}$-Facetten. Um die Drähte in Bezug auf die Größe einfacher vergleichen zu können, wurde ein äquivalenter Durchmesser, der dem Durchmesser eines Kreises mit der gleichen Fläche wie des facettierten Querschnitts entspricht, verwendet. Dieser liegt bei den Drähten zwischen 40 und 300nm.

Um die Nanodrähte vom Substrat, auf dem sie gewachsen sind, zu den Zugapparaturen zu transferieren, wurde ein Transferprozess entwickelt, der auf der Verwendung eines Mikromanipulators (bisher wurden Manipulatoren der Firmen Omniprobe und Kleindiek Nanotechnik erfolgreich eingesetzt) und eines Gas-Injektions-Systems für Platindeposition mit Hilfe des Elektronenstrahls in der Kammer eines REMs basiert. Der Transferprozess an sich ist für beide Zugapperaturen nahezu identisch. Im Fall der Zugapperatur für das REM ist allerdings zusätzlich im Vorfeld die Spitze des Kraftsensors und das TEM-Halbmondgrid mit Hilfe der Positionierer zueinander in Höhe (z) und lateraler Position (y) exakt auszurichten. Die Lücke zwischen TEM-Grid und Spitze des Sensors bestimmt dabei die Messlänge des Drahtes und wird für die Zugversuche hier mit Hilfe des x-Positionieres auf circa $9 \mu m$ gesetzt.

Der erste Schritt des Transfers besteht darin, einen Draht mit der gewünschten Länge, wobei man hier ungefähr $3 \mu m$ für den Prozess hinzuaddieren muss, und dem gewünschten Durchmesser auf dem Substrat auszuwählen. Die Spitze des Mikromanipulators wird nun in Kontakt mit der Wurzel des Drahtes gebracht. Hierfür kann man die Translation und Rotation des Probentisches sowie die Bewegung der Manipulator- 


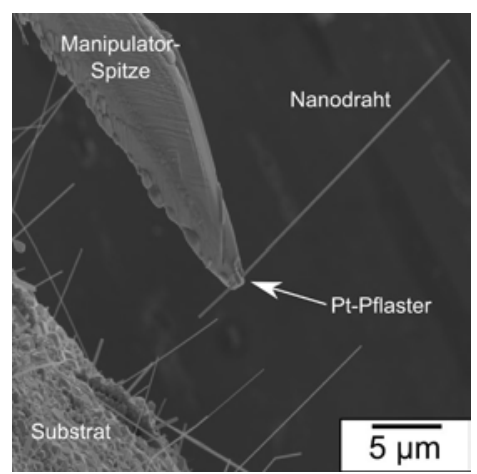

(a)

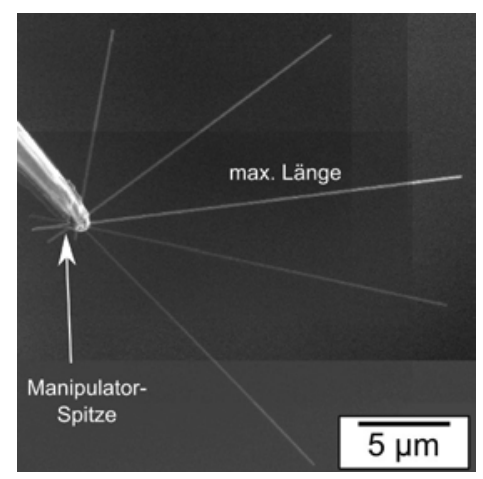

(b)

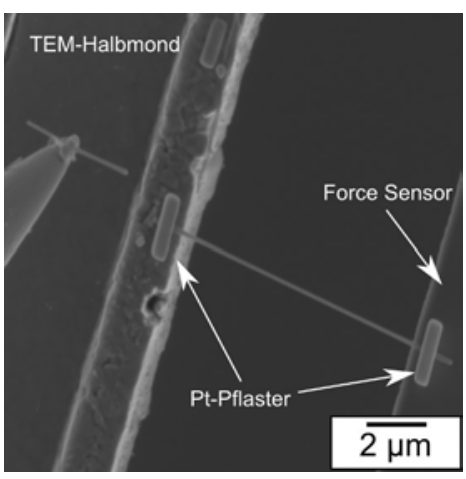

(c)

Abbildung 3.3.: Transferprozess: (a) Lift-Off des Drahtes vom Substrat; (b) Ausrichten des Drahtes; (c) Befestigung des Drahtes auf der Zugapparatur

spitze verwenden. Der Draht wird dann mittels elektronenstrahl-deponiertem Platin an den Manipulator geklebt, wobei das Klebepflaster die typischen Dimensionen von circa 800nm x 200nm mit einer Dicke von 500nm aufweist. Entfernt man danach den Manipulator langsam vom Substrat, kommt es zum Riss zwischen Draht und Substrat. Das Pflaster hält dabei den Draht steif am Manipulator fest (siehe Abbildung 3.3). Dieser Vorgang lässt das freistehende Ende das Drahtes unverformt. Dies wurde überprüft, indem die Manipulatorspitze mit einem befestigten Nanodraht in einen TEM-Halter eingebaut wurde. Hierbei ließen sich keine Defekte im Draht feststellen.

Im nächsten Schritt wird die Manipulatornadel um ihre Achse rotiert (siehe Abbildung 3.3) und die projektierte Länge des Drahtes in den REM-Bildern gemessen. Es wird angenommen, dass der Draht genau dann in der Bildebene liegt, wenn die projektierte Länge ihr Maximum erreicht. Die Zugapparatur oder das CuBe-Grid werden nun mit Hilfe von Translation und Rotation des Proben-Tisches so unterhalb des Drahtes positioniert, dass Drahtachse und Zugachse bzw. Gridachse parallel zueinander ausgerichtet sind. Der Draht wird danach entweder durch langsames Anheben des Probentisches oder Absenken des Drahtes mit der jeweiligen Zugapparatur in Kontakt gebracht. Der Kontaktpunkt ist dabei durch ein leichtes Biegen des Drahtes und durch eine Veränderung des Vibrationsverhalten des Drahtes auszumachen. Der Draht wird anschließend durch zwei Platinpflaster an dem Kraftsensor und dem Aktuator bzw. auf beiden Seiten des Fensters auf dem CuBe-Grid befestigt. Im letzten Schritt erfolgt das Zurückziehen der Manipulator-Nadel, so dass der Draht zwischen der Klebestelle zum Manipulator und der Platinhalterung reißt. Das Drahtsegment zwischen den beiden 
Halterungen bleibt dabei unverformt und der Draht ist nun fertig zum mechanischen Testen (siehe Abbildung 3.3).

\subsubsection{Ablauf im TEM}

Nach dem Transfer des Drahtes über das Fenster einer CuBe-Folie kann diese in den Zughalter eingebaut werden. Nach dem Einbau des Zughalters ins TEM weist der Draht typischerweise zunächst eine elastische Verbiegung auf, was sowohl an einer nicht gerade gespannten Form des Drahtes als auch an den Biegekonturen entlang des Drahtes zu erkennen ist. Der Draht wird nun vorsichtig mit kleinen Dehnungsschritten in eine gerade Form gebracht und auf vorhandene Defekte untersucht. Als Zonenachse wurde dazu eine möglichst niedrig indizierte Zonenachse durch Verkippen des Halters verwendet, vornehmlich eine [110] oder [111]-Zonenachse, sofern dies möglich war. Die Zonenachse ließ sich auch während des Zugversuchs ändern, allerdings wurde häufiges Kippen des Halters während der Zugversuche vermieden, um ein Reißen des Drahtes durch äußere Vibrationen beim Kippen zu vermeiden. Im weiteren Verlauf wird der Draht mit den kleinstmöglichen Schritten weiter gedehnt und während der Verformung kontinuierlich von der CCD-Kamera aufgenommen. Die Vergrößerung wurde dabei so gewählt, dass sie zum einen nicht zu klein ist, um das Entstehen von Defekten nicht zu verpassen, zum anderen nicht zu groß, um so ein möglichst großes Blickfeld entlang des Drahtes zu erhalten. Bei vollem Videospeicher wurde die Verformung unterbrochen und die Bilder gespeichert. Diese Unterbrechungen wurden dabei teilweise genutzt, um den gesamten Draht in mehreren Bildern detailreich abzubilden, die Zonenachse zu wechseln oder auch die Richtung der Verformungsschritte zu ändern und den Draht wieder zu entlasten. Schließlich wird der Draht in mehreren solcher Schritte bis zum Bruch verformt.

\subsubsection{Ablauf und Messgrößen im REM}

Nachdem ein Draht zwischen Kraftsensor-Spitze und TEM-Halbmond auf dem Aktuator befestigt ist, erfolgt das mechanische Testen durch Anlegen einer konstanten Verformungsrate während der Draht mittels Elektronenstrahl abgebildet und die Kräfte aufgenommen werden. Die Verformungsraten liegen ungefähr zwischen 0, $5 \mathrm{~nm} / \mathrm{s}$ und $3 \mathrm{~nm} / \mathrm{s}$, so dass die Dehnraten für einen typischen Draht mit einer Länge von $9 \mu \mathrm{m}$ im 
Bereich von $10^{-5} / \mathrm{s}$ bis $10^{-4} / \mathrm{s}$ liegen.

Die Dehnungen, die der Draht im Zuge der Verformung erfährt, werden mit Hilfe von Digital Image Correlation [53] aus den REM-Bildern gewonnen, die fortlaufend während des Zugversuchs gespeichert werden. Hierbei werden die beiden PlatinHalterungen, die einen starken Kontrast haben, verwendet, um die Bewegung der beiden Enden eines Drahtes zu verfolgen. Die Verlängerung in x-Richtung, also entlang der Aktuatorachse, und der Achse des Kraftsensors wird schließlich durch die ursprüngliche Drahtlänge dividiert, um so die technische Dehnung zu erhalten. Die Ausgangslänge des Drahtes wird dabei zu dem Zeitpunkt der Verformung bestimmt, in dem die Kraft auf den Draht anfängt zu steigen, nachdem jede Krümmung aus dem Draht verschwunden ist. Falls der Draht nach dem Transfer bereits gerade sein und damit eventuell unter Zugspannung stehen sollte, wird der Aktuator vorsichtig in Richtung des Kraftsensors bewegt bis sich der Draht leicht biegt und sich der Anfangspunkt für Kraft und Länge präzise bestimmen lässt. Die laterale oder y-Verschiebung der beiden Klebestellen zueinander war in der Regel kleiner als 2 Pixel, was ungefähr 20nm entspricht und damit mindestens um den Faktor 10 kleiner ist als der Verfahrweg in Richtung der Zugachse. Diese Beobachtung genauso wie die Tatsache, dass die Drähte in der Mitte versagen, spricht für kleine Biegemomente und Lateralkräfte sowie eine gute Ausrichtung zur Zugachse während der Verformung.

Um ein mögliches Rutschen unter den Platin-Klebestellen auszuschließen, wurde ein Kontrolltest durchgeführt, bei dem die Dehnung wie gewöhnlich zwischen den beiden Platinpflastern mit der Dehnung zwischen zwei kleinen Platinmarkern verglichen wurde, die direkt auf dem Draht deponiert waren. Abbildung 3.4 zeigt einen solchen Draht, bei dem zunächst der Aktuator in Richtung des Kraftsensors bewegt und der Draht leicht gebogen wurde, um den Anfangspunkt zu bestimmen. Danach wird der Draht um ungefähr 1\% gedehnt, danach wieder entladen und schließlich bis zum Versagen gedehnt. Ein Vergleich der beiden Dehnungsmessungen zeigt keinen signifikanten Unterschied mit Ausnahme während der anfänglichen Biegung des Drahtes.

Die Genauigkeit, mit der sich die Dehnung bestimmen lässt, ist weitestgehend von der Qualität der REM-Bilder abhängig. Zusätzlich zu einer Optimierung von Kontrast und Helligkeit der Bilder muss man einen Kompromiss zwischen Pixelgröße und DwellTime finden. Für diese Arbeit wurde daher eine Pixelgröße von circa 10nm gewählt, wobei die gesamte Drahtlänge mit einer Auflösung von 1024 x 943 (bei neuen Drähten 


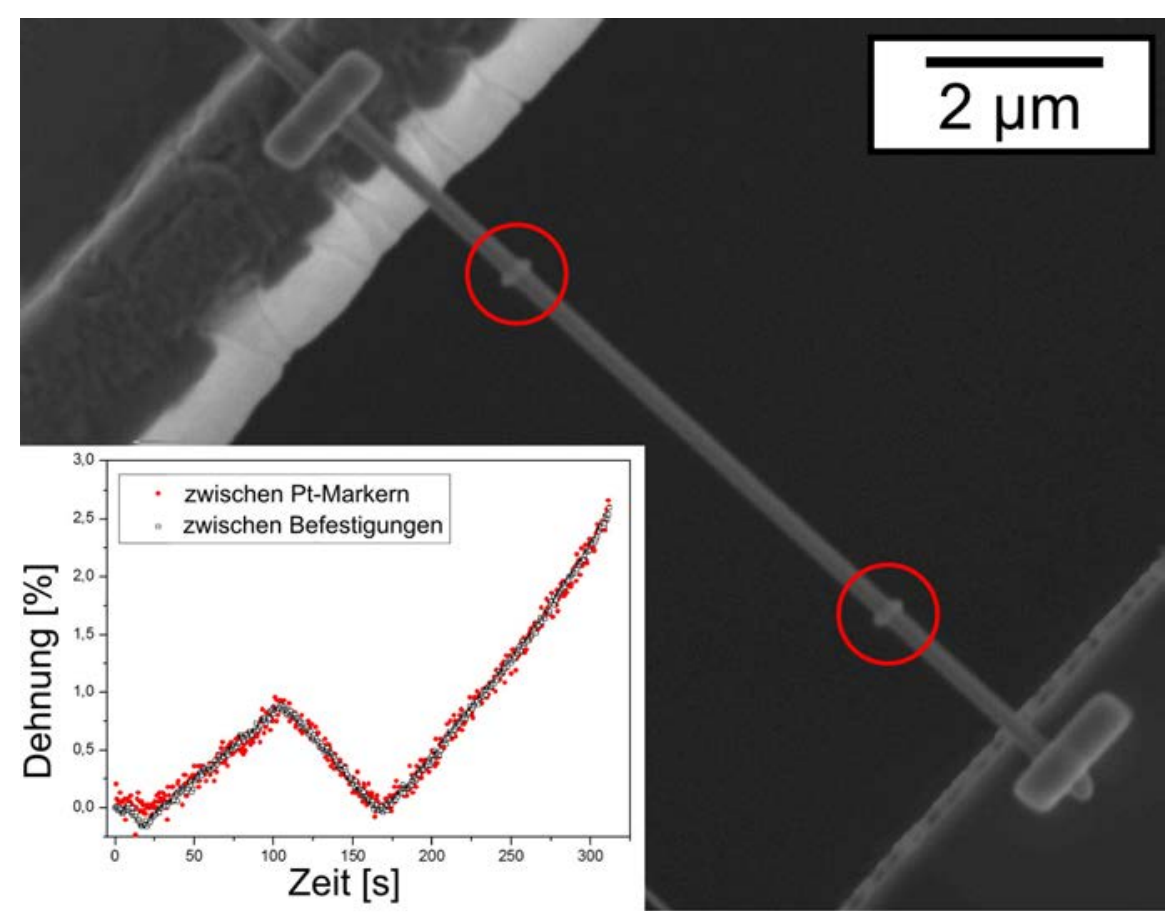

Abbildung 3.4.: Marker-Experiment zur Kontrolle der Dehnung: Die REM-Aufnahme zeigt einen transferierten Draht mit zwei zusätzlichen Pt-Markern (rote Kreise). Das Inset zeigt die Dehnung gegen die Zeit sowohl zwischen den Befestigungen als auch zwischen den Markern.

mit 1240 x 943, bedingt durch ein Update der REM-Software) abgebildet wurde. Dadurch lag die Framerate zwischen 1 und 2 Frames pro Sekunde und für die DIC-Analyse ließ sich eine Genauigkeit von ungefähr $\pm 2 n m( \pm 0,2$ pixeln $)$ realisieren. Damit ergibt sich für die Genauigkeit der Dehnungsbestimmung ein Intervall von ungefähr $0,02 \%$.

Die Kraft, die durch die Elongation des Drahtes hervorgerufen wird, führt zu einer kleinen Verschiebung in der MEMS-Struktur des Kraftsensors, die durch die Steifigkeit des Sensors bestimmt ist. Das resultierende Spannungssignal wird in der integrierten Elektronik des Sensors verstärkt und anschließend mit einem Umrechnungsfaktor in eine Kraft übersetzt, die mit einer Aufnahmerate zwischen $1 \mathrm{~Hz}$ und $10 \mathrm{~Hz}$ mittels eines auf Labview basierten Programms aufgezeichnet wird. Um die Auflösung und Stabilität des Sensors in der Vakuumkammer des REMs zu testen, wurde die Kraft auf den ungeladen Sensor für 60 Minuten aufgenommen, einmal mit Elektronenstrahl und einmal ohne Elektronenstrahl (siehe Abbildung 3.5), sowie direkt nach dem Einbau als auch mit einer Verzögerung von mehr als 12 Stunden. 60 Minuten entsprechen dabei dem oberen Limit für die Dauer eines 'normalen' Tests. Entlastungsversuche können bis zu 120 Minuten dauern. 


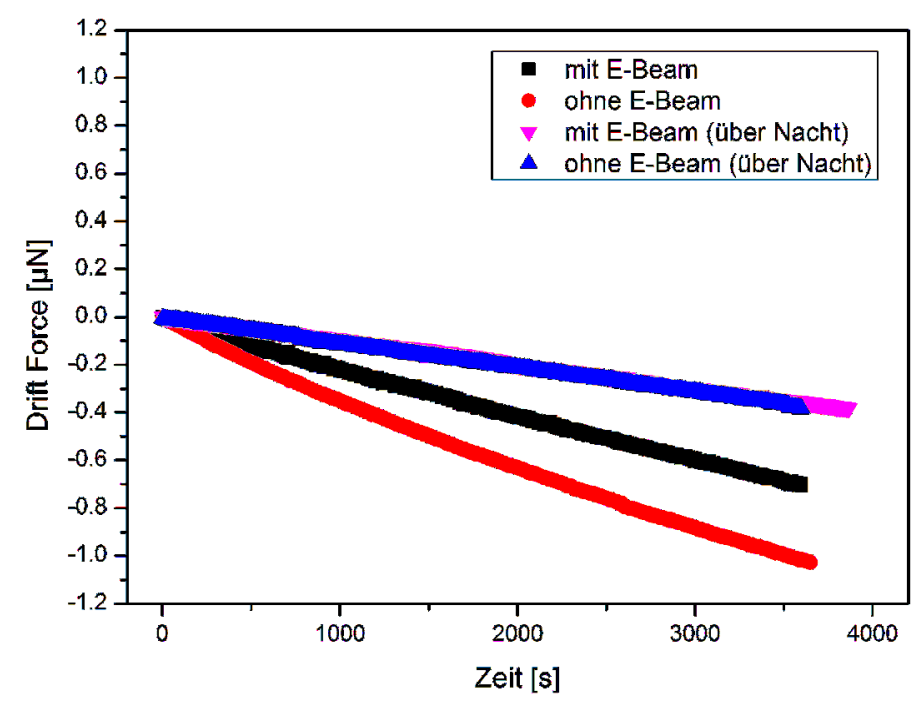

Abbildung 3.5.: Drift-Kraft gegen die Zeit mit und ohne Elektronenstrahl sowie direkt nach dem Einbau als auch mit einer Verzögerung von >12Stunden

Das Rauschen in der gemessen Kraft liegt bei $\pm 5 n N$ und befindet sich damit in guter Übereinstimmung mit den Spezifikationen des Herstellers. Die Drift liegt für den hier getesteten Sensor immer in negativer Richtung und verläuft über den gesamten Zeitraum der Messung annähernd linear (eine Messung 'ohne E-Beam' zeigt die größte Abweichung von der Linearität). Dabei erreicht die Drift im ungünstigsten Fall Werte von $1 \mu N$. Rechnet man diese Kräfte in eine Spannung um für den kleinsten getesteten Draht mit einem äquivalenten Durchmesser von 60nm, erhält man eine Spannungsauflösung von $\pm 2 M P a$ und maximale Drift der Spannungswerte um 350MPa. Vergleicht man die Drift-Kurven direkt nach dem Einbau mit den Drift-Kurven, die nach einer Wartezeit von mehr als 12 Stunden aufgenommen wurden, so erkennt man eine deutliche Reduktion der Drift-Werte. Hier erreicht die Drift Werte von 0, $4 \mu N$ entsprechend einer Spannung von 140MPa. Ein Grund für die kleineren Drift-Werte mag an der Stabilität des Vakuums liegen. Dementsprechend wurde, sofern möglich, das komplette Setup mindestens 12 Stunden vor den Versuchen eingebaut. In beiden Fällen, also direkt nach dem Einbau als auch nach der Wartezeit, ließ sich kein Einfluss des Elektronenstrahls auf die Drift feststellen.

Da vor dem Versuch noch keine Zugkraft sondern nur eine minimale Druckkraft durch die Biegung des Drahtes und nach dem Reißen überhaupt keine Kraft auf den Sensor wirken, ist es möglich, die Kraftdaten während des Versuchs um den Einfluss 


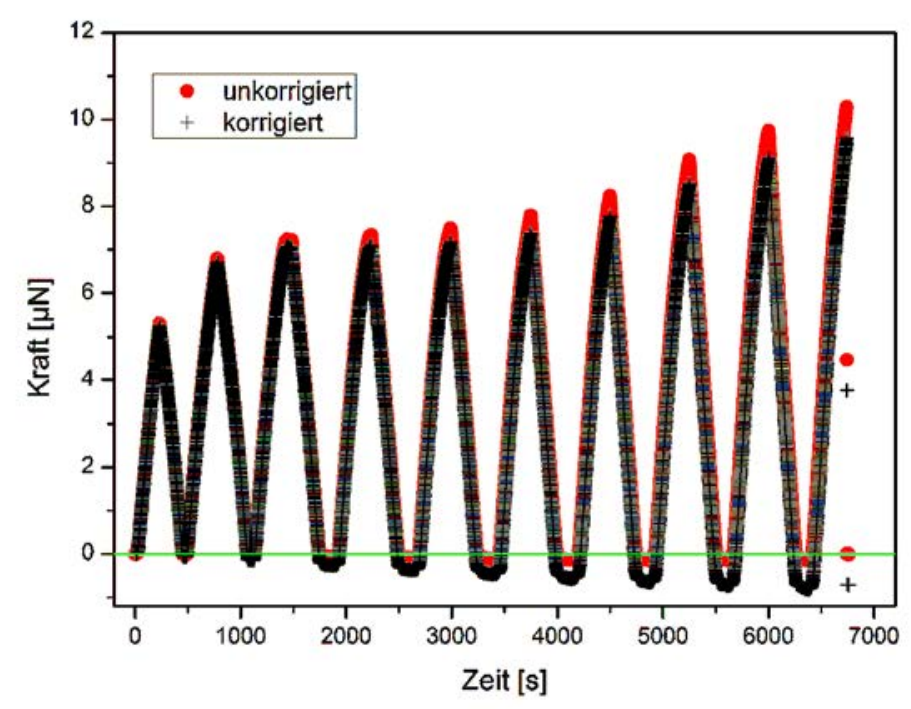

Abbildung 3.6.: Kraft gegen Zeit für einen Entlastungsversuch sowohl ohne Driftkorrektur (schwarzes Plus) als auch mit Driftkorrektur (roter Punkt). Die grüne Linie zeigt die Nulllinie der Kraft an.

der Drift während eines Versuchs zu korrigieren. Dazu wird eine lineare Drift während des Tests angenommen und die Kraftdaten dementsprechend korrigiert. Die Annahme einer linearen Drift lässt sich zum einen mit den Driftmessungen aus Abbildung 3.5 rechtfertigen. Zum anderen kann man die Entlastungsversuche heranziehen. Die Zeit-Kraft-Kurve für einen solchen Versuch mit mehreren Entlastungssegmenten ist in Abbildung 3.6 sowohl für die unkorrigierten als auch für die drift-korrigierten Daten dargestellt. Der Draht wurde hierbei jeweils soweit entlastet, dass er eine kleine Biegung aufwies, so dass auf den Kraftsensor nur eine minimale Kompressionskraft in der Nähe der Nulllinie (grüne Linie in Abbildung 3.6) wie zu Beginn des Tests wirken sollte. Man erkennt, dass dies für die korrigierten Werte sehr gut der Fall ist, während die unkorrigierten Werte mit der Zeit immer weiter abweichen.

Um den Umrechnungsfaktor zum Bestimmen der Kraft zu kontrollieren, den der Hersteller für jeden Sensor individuell bereitstellt, wurden unter einem Lichtmikroskop (nicht möglich im REM, da nur die Durchführung für einen einzelnen Kraftsensor vorhanden ist) zwei Sensoren mit Hilfe des Zugaufbaus für das REM gegeneinander getestet (siehe Abbildung 3.7). Dazu erfolgte die Montage eines zweiten Sensors anstelle des Halbmondgrids auf dem z-Positionierer. Die beiden Spitzen der Sensoren wurden dabei wiederherum mit Hilfe der drei Positionierer zueinander ausgerichtet 
und schließlich mit dem x-Positionierer gegeneinander gedrückt, wobei die Kraft der beiden Sensoren aufgenommen wurde. Dies ließ zwar den Vergleich zwischen zwei Sensoren zu, jedoch keine Überprüfung der absoluten Werte für den Umrechnungsfaktor. Hierbei hat sich gezeigt, dass unter der Verwendung der vom Hersteller gelieferten Umrechnungsfaktoren Differenzen in der gemessenen Kraft zwischen zwei Sensoren von bis zu 30\% auftreten können (siehe Abbildung 3.7).

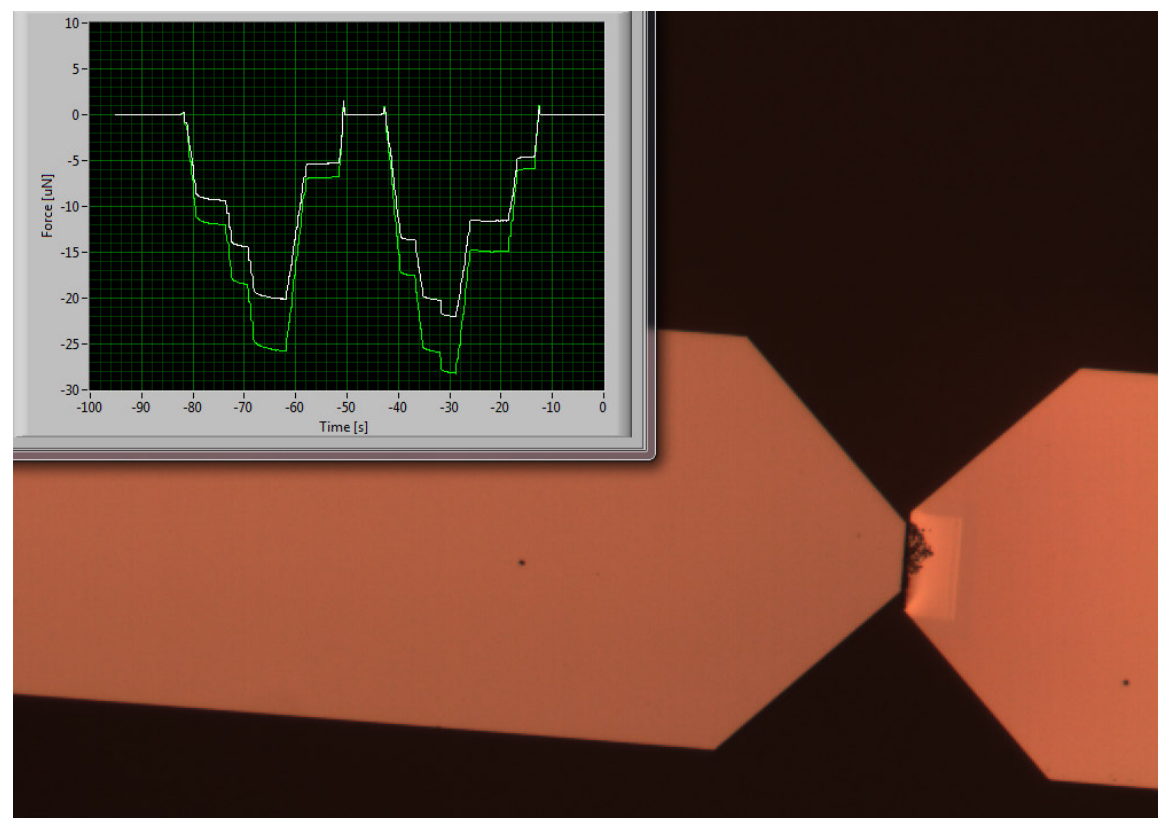

Abbildung 3.7.: Kontrolle der Kraftsensor-Kalibrierung: Lichtmikroskop-Aufnahme von zwei Sensor-Spitzen sowie die Kraft-Zeit-Daten der beiden Sensoren, die einen deutlichen Unterschied zeigen

Um den absoluten Wert für den Umrechnungsfaktor bestimmen zu können, kamen zwei Methoden zur Anwendung. Hierbei wird der Umrechnungsfaktor zum einen mit Hilfe eines Cantilevers im REM bestimmt. Dazu wird zunächst die Federkonstante des Cantilevers im AFM über ein thermisches Anregungsspektrum bestimmt und der Cantilever anschließend auf dem z-Positionierer befestigt. Der Cantilever wird dann vorsichtig mit dem x-Positionierer gegen den Kraftsensor gefahren, so dass es zur Biegung des Cantilevers kommt. Mit der bekannten Federkonstante des Cantilevers sowie dem Verfahrweg während der Biegung, der wiederherum mit Digital Image Correlation bestimmt wurde, lässt sich die Kraft des Cantilevers ausrechnen und damit die Kalibrierung des Sensors überprüfen. Da sich die Federkonstante des Cantilevers mit der gewählten Methode nur mit einer Genauigkeit zwischen 10\% und 20\% ermitteln lässt [54], wurde zusätzlich mit einer weiteren Methode, der absolute Wert des Umrechnungs- 
faktors bestimmt. Dazu wurde ein Kraftsensor derart in einen Nanoindenter (Agilent G200) eingebaut, dass die Nanoindenter-Achse und Kraftsensor-Achse parallel zueinander liegen. So konnte die Spitze des Nanoindenters gegen die Spitze des Kraftsensors gedrückt und die gemessenen Kräfte miteinander verglichen werden. Der so erhaltene Wert für den Umrechnungsfaktor scheint damit am zuverlässigsten zu sein, da der Nanoindenter eine spezifizierte Kraftauflösung von 1nN aufweist [55]. Wegen des Einsatzes mehrerer Sensoren im Rahmen dieser Arbeit wurde der Umrechnungsfaktor des jeweilig verwendeten Sensors derart angepasst, dass er mit der Nanoindenter-Messung übereinstimmte. Da nur ein Sensor mit Hilfe des Nanoindenters kalibriert wurde, wurden die Vergleichsmessungen unter dem Lichtmikroskop zur Umrechnung herangezogen.

Die Umrechnung der Kraft des Sensors in eine Spannung ließ sich schließlich durch Messen der Querschnittsfläche des Drahtes vollziehen. Nach dem Test wird dazu mit dem FIB ein Schnitt in eine der beiden Platinbefestigungen gemacht. Ein REM-Bild der Querschnittsfläche lässt dann eine Bestimmung der Querschnittsfläche zu. Da das REM allerdings Probleme hat, Kanten und Rundungen exakt abzubilden, war es schwer, eine genaue Abgrenzung zwischen Drahtfläche und umgebenden Platin zu bestimmen, so dass eine präzise Flächenbestimmung aus dem REM-Bild schwierig ist. Deshalb und auch zur Bestimmung, ob der Draht über einen Wachstumszwilling verfügt, wurde von vielen Drähten eine TEM-Lamelle durch eine der beiden Klebestellen angefertigt. Die Flächenbestimmung konnte dann in Transmissionsbildern vorgenommen werden, in denen der Kontrast von Draht zum umgebenden Platin viel deutlicher war.

\subsection{Erzeugen von Defekten}

In diesem Abschnitt erfolgt die Darstellung der Methoden, die benutzt wurden, um Defekte vor dem eigentlichen Zugversuch in den Draht einzubringen. Ziel war es dabei, den Draht möglichst in seiner ursprünglichen Form zu belassen. Gleichzeitig musste es nach dem Einbringen der Defekte noch möglich sein, den Draht auf das gewünschte Test-Setup transferieren zu können. Insgesamt wurden drei verschiedene Methoden verwendet: Das Biegen bzw. Quetschen im REM, eine recht schnelle Methode, die aber sehr schwierig zu kontrollieren ist; die 3-Punkt-Biegung im TEM oder REM, eine Methode die kontrollierbarer ist, aber auch experimentell aufwendiger; die Indentierung mit dem AFM, die sich letztlich als experimentell zu schwierig erwiesen hat und deshalb 
nicht weiter verfolgt wurde.

\subsubsection{Biegen und Quetschen}

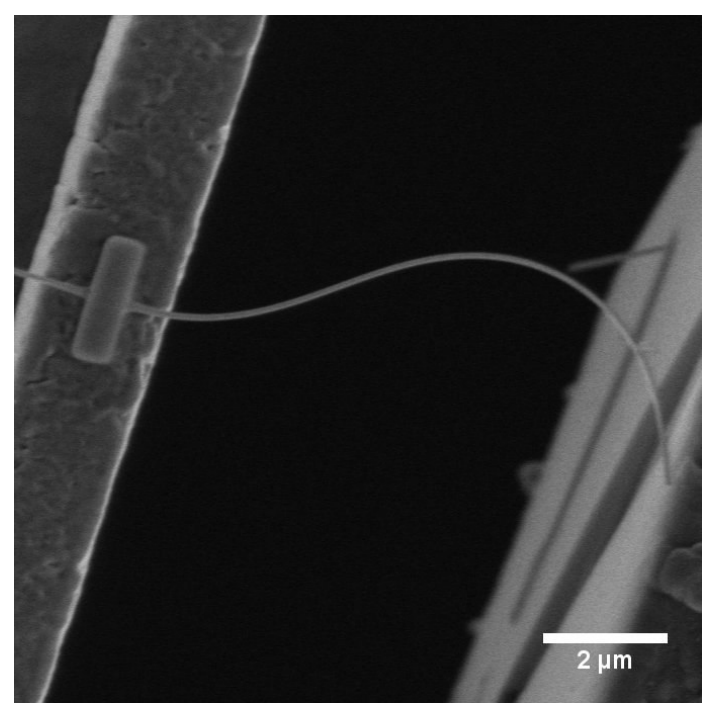

(a)

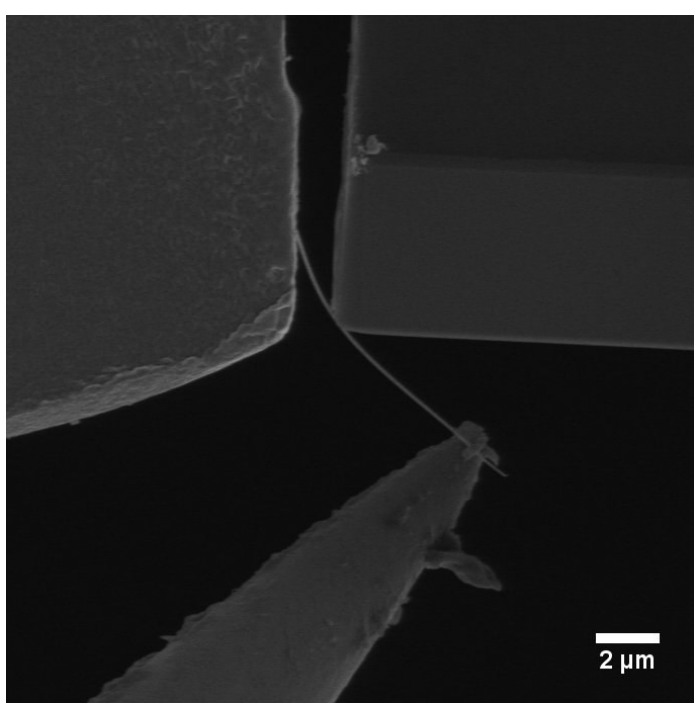

(b)

Abbildung 3.8.: Biegen und Quetschen im REM

Zum Biegen und Quetschen findet der Zugaufbau für das REM Verwendung. Es wird zunächst wie gewöhnlich ein Draht mit Hilfe eines Mikromanipulators vom Substrat abgeholt. Im Falle des Biegens wird dieser anschließend an ein vorpräpariertes Halbmondgrid transferiert und befestigt. Der Draht muss hierbei zur Unterstützung am Ende auf einer Fläche aufliegen, da die Platindeposition in der Nähe des Manipulators ohne Unterstützung des Endes zu einer Verbiegung des Drahtes führen kann. Anschließend lässt sich der freistehende Draht mit Hilfe des x-Positionieres gegen eine Fläche bewegen, so dass es zur Biegung des Drahtes kommt (siehe Abbildung 3.8). Als Fläche eignet sich beispielsweise das Ende des Kraftsensors. Der Draht kann nun soweit und sooft gegen die Fläche bewegt werden, bis eine Verformung des Drahtes zu beobachten ist. Die Position bzw. der Ort der Verformung entlang des Drahtes ließ sich dabei steuern und trat nicht unbedingt an dem Punkt der maximalen Biegung auf. Anschließend konnte das Halbmondgrid ins TEM eingebaut werden, um so die entstandenen Defekte zu analysieren. Um abschließend einen Zugversuch mit dem Draht durchzuführen, wurde im Fall eines REM-Tests das freistehende Ende an der Spitze des Kraftsensors befestigt. Im Fall eines TEM-Tests ist ein zweiter Transfer nötig. Dazu 
ist es erforderlich, den Draht analog zum Abholen vom Substrat nahe der Klebestelle an dem Halbmondgrid an dem Manipulator mit Platin zu befestigen und anschließend in identischen Schritten wie bei den schon beschriebenen Transfers auf ein CuBe-Grid zu platzieren.

Zum Quetschen des Drahtes wird ebenfalls der Zugaufbau verwendet. Hierbei wird der Draht nach dem Abholen vom Substrat zunächst an dem Manipulator belassen und zwischen zwei Flächen verfahren. Hierzu können beispielsweise erneut das Ende eines Halbmondgrids und das Ende der Kraftsensorspitze dienen.

\subsubsection{3-Punkt-Biegung}

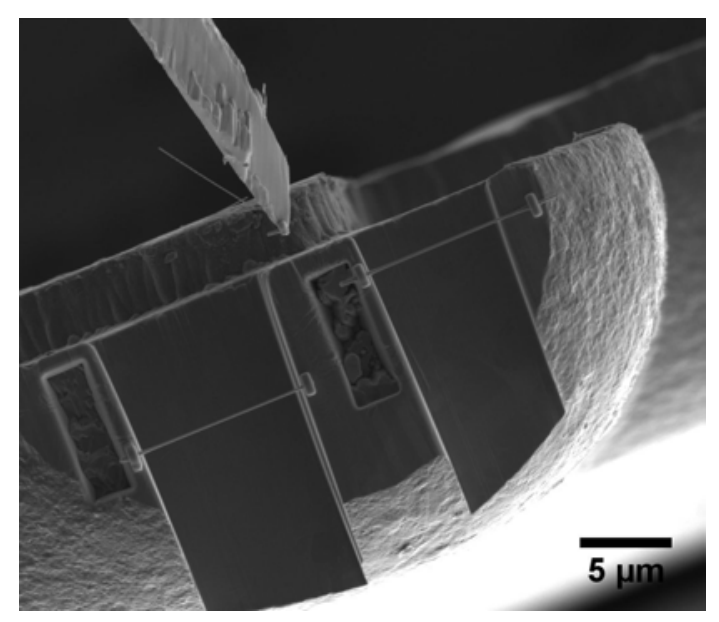

Abbildung 3.9.: 3-Punkt-Biegung Vorbereitung: Zwei Drähte wurden zur 3-PunktBiegung über Gräben auf ein Halbmondgrid transferiert.

Für die 3-Punkt-Biegung wird zunächst ein TEM-Halbmond-Grid vorstrukturiert und anschließend ein Draht auf das vorstrukturierte Grid transferiert. Abbildung 3.9 zeigt sowohl das präparierte Halbmondgrid als auch zwei transferierte Drähte. Hierzu wird zunächst der Steg eines Halbmondgrids senkrecht mit Hilfe des FIB's abgeschnitten, so dass eine flache Kante entsteht. Anschließend wird ein Graben mit einer Breite um $8-9 \mu m$ in die flache Kante geschnitten. Über diesen Graben können in einem typischen Transferprozess Drähte mit Hilfe eines Mikromanipulators und Platinabscheidung befestigt werden. Die Geometrie ist dabei so gewählt, dass das Grid nach der Biegung ins TEM eingebaut werden kann, um zu prüfen, welche Defekte durch die Biegung erzeugt wurden.

Die 3-Punkt-Biegung erfolgt anschließend mit Hilfe der Zugapparatur. Dazu wird 


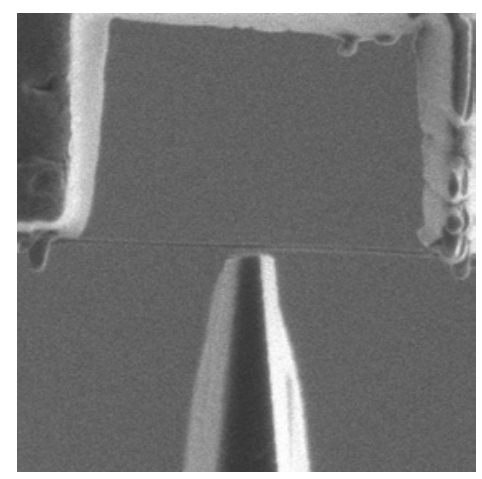

(a)

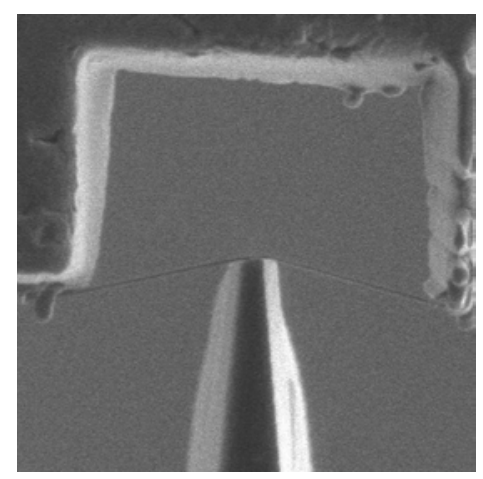

(b)

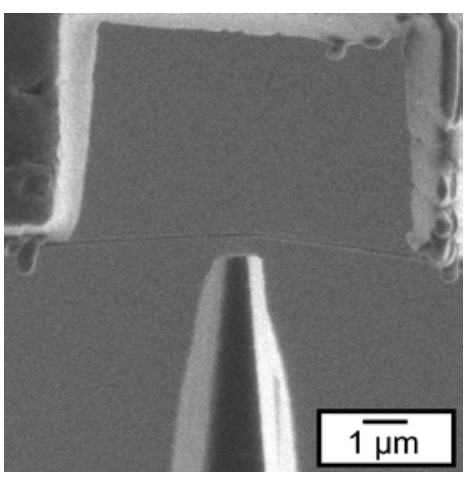

(c)

Abbildung 3.10.: 3-Punkt-Biegung

die Spitze eines Kraftsensors mit dem FIB dahingehend modifiziert, dass man eine Art Indenter-Keil erhält (siehe Abbildung 3.10 a). Das TEM-Grid mit dem Draht über dem Graben wird nun vorsichtig gegen den Indenter-Keil bewegt, so dass es zur Biegung des Drahtes kommt (siehe Abbildung 3.10 b). Während der Biegung kann die Kraft auf den Sensor aufgenommen werden. Die Biegung kann nun so weit durchgeführt werden bis eine kleine permanente Verformung des Drahtes sichtbar ist (siehe Abbildung 3.10 c). Anschließend lässt sich im TEM untersuchen, welche Defekte im Draht entstanden sind.

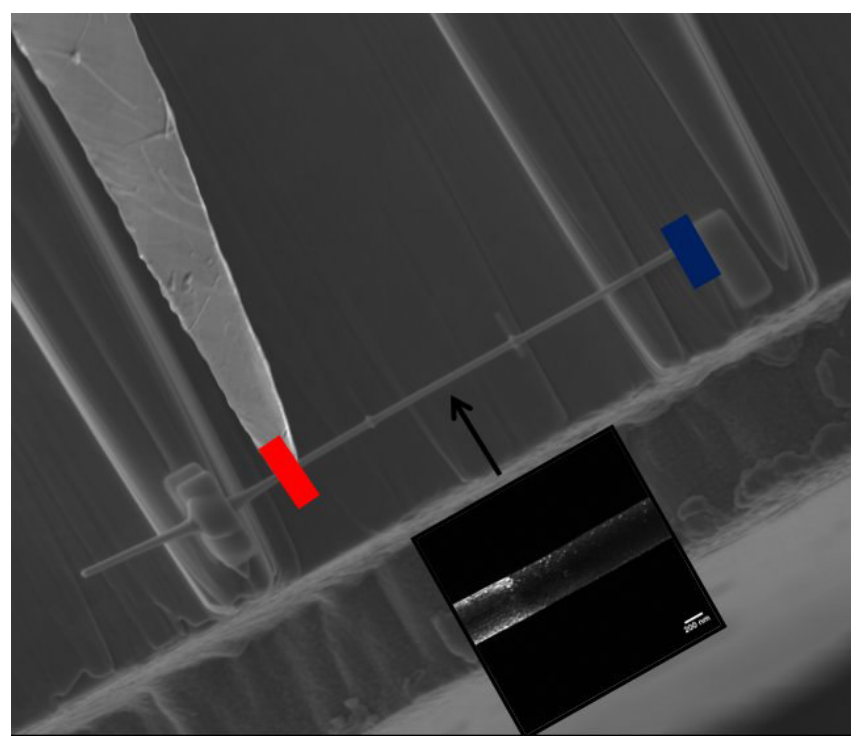

Abbildung 3.11.: Zweiter Transfer für einen vorverformten Draht

Um einen derart vorverformten Draht für einen Zugversuch verwenden zu können, ist ein zweiter Transferprozess erforderlich. Dazu wird der Mikromanipulator unterhalb 
des Drahtes in die Nähe eines der beiden Platinbefestigungen gebracht und der Draht erneut mit Platin an der Nadel befestigt (rot markiert in Abbildung 3.11). Anschließend wird der Draht von der gegenüberliegenden Platinbefestigung mit Hilfe des Ionenstrahls getrennt (blau markiert in Abbildung 3.11). Hierbei ist darauf zu achten, dass mit dem Ionenstrahl lediglich die Klebestelle bzw. ein minimaler Teil des Drahtes bestrahlt wird. Der nun an dem Manipulator freistehende Draht kann mit den üblichen Methoden auf ein $\mathrm{CuBe}-$ Grid oder auf die Zugapparatur transferiert werden. Um besser zuordnen zu können, wo genau die eingebrachten Defekte entlang des Drahtes liegen, lassen sich zur Orientierung auch Platin-Marker auf dem Draht deponieren. 


\section{Experimentelle Ergebnisse}

Dieses Kapitel widmet sich der Vorstellung der experimentellen Ergebnisse dieser Arbeit. Dazu gehört zunächst eine Charakterisierung der Proben, in der zwischen einkristallinen Proben und Proben mit longitudinaler Zwillingsgrenze unterschieden wird. Anschließend wird für beide Probentypen separat auf das Verformungsverhalten und die Entwicklung der Mikrostruktur eingegangen. Danach folgt die Präsentation der Spannungs-Dehnungs-Kurven. Mit Ausnahme bei der Probencharakterisierung werden die Kapitel mit einer Zusammenfassung der wichtigsten Aspekte des jeweiligen Ergebnisteils abgeschlossen.

\subsection{Charakterisierung der Proben}

\subsubsection{Einkristalline Proben}

Zur Charakterisierung der Proben konnten zum einen die Querschnittsbilder der Drähte herangezogen werden. Dazu zählen sowohl die Querschnittsbilder, erstellt mit dem FIB, als auch die Bilder der Querschnittslamellen aufgenommen im TEM. Bei den Lamellen lässt sich die Wachstumsrichtung der Drähte relativ problemlos aus dem Beugungsbild bestimmen. In Abbildungen 4.1 (a) ist die Hellfeldaufnahme einer Querschnittslamelle zu sehen. Abbildungen 4.1 (b) zeigt das zugehörige Beugungsbild. Man erkennt deutlich die $<110>$-Zonenachse, die folglich auch der Wachstumsrichtung des Drahtes entspricht. Dies war der Fall für alle getesteten Drähte.

Die Querschnittsform der Drähte ist immer stark facettiert. Üblicherweise dominieren vier Facetten. Aus den Winkeln und der bekannten Drahtachse entlang der $<110>$-Richtung kann man auf \{111\}-Facetten schließen. Auf den Querschnittsbildern ist weiterhin erkennbar, dass die Facetten nicht perfekt ausgebildet sind, das heißt an den Übergängen zwischen den Facetten kommt es zur Abrundung. Der Radius der Abrundung liegt etwa zwischen $5 \mathrm{~nm}$ und 10nm. Teilweise werden die spitzen Ecken in der 


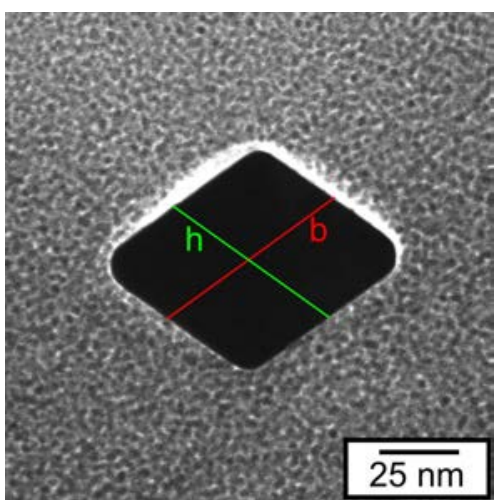

(a)

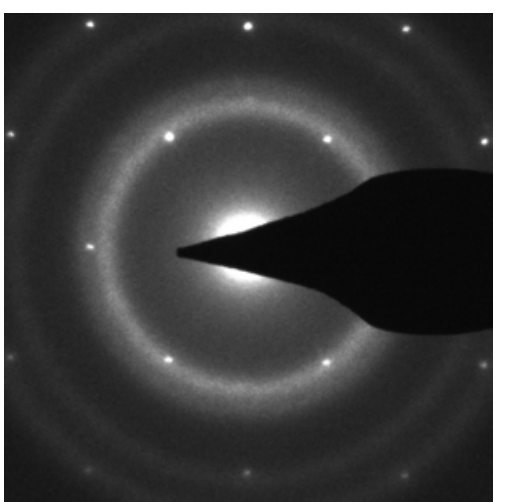

(b)

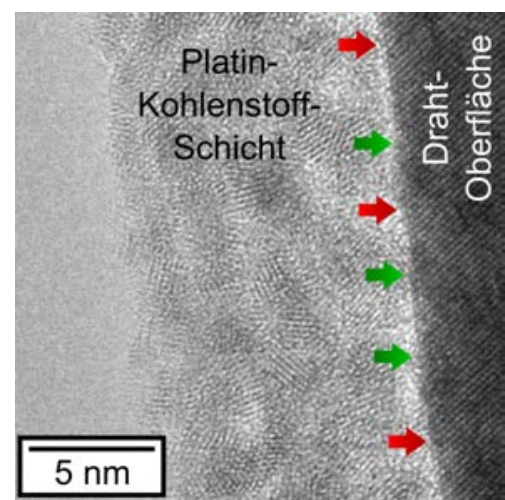

(c)

Abbildung 4.1.: Charakterisierung von Drähten: (a) zeigt die TEM-Hellfeldaufnahme einer Querschnittslamelle, (b) zeigt das dazugehörige Beugungsbild und (c) zeigt die Hochaufösungsaufnahme einer Drahtoberfläche mit Platin-Kohlenstoff-Schicht.

Weise abgerundet, dass weitere Facetten entstehen, bei denen es sich um $\{100\}$-Facetten handelt. Eine solche Querschnittsform, also ein Rhomboid aus $\{111\}$-Facetten mit abgestutzten Ecken und kleinen $\{100\}$-Facetten, wird auch aus einer Wulf-Konstruktion für einen Gold-Kristall mit <110>-Achse erwartet. Dabei kann die Ausprägung der beiden $\{100\}$-Facetten unterschiedlich stark sein und so zu einer unsymmetrischen Querschnittsform des Drahtes führen.

Der Durchmesser der Drähte variiert zwischen 60 und 150nm. Hierbei wurde die ausgemessene Querschnittsfläche der Drähte als Kreis angenommen, so dass man richtigerweise von einem äquivalenten Durchmesser sprechen sollte. Durch die Abrundung der Ecken war es nicht möglich, eine genaue Facettenlänge zu bestimmen. Stattdessen wurde jeweils die Breite $b$ und Höhe $h$ der Drähte, wie sie in Abbildung 4.1 (a) eingezeichnet sind, ausgemessen. Diese liegen zwischen 20 und 330nm. Das Aspektverhältnis von Höhe zu Breite variiert zwischen 1 und circa 7. Der Großteil der einkristallinen Drähte liegt unterhalb eines Aspektverhältnisses von 3.

Neben den Querschnittsbildern können die TEM-Aufnahmen, die vor dem Testen gemacht wurden, zur Charakterisierung der Nanodrähte herangezogen werden. Die Oberfläche entlang der Drähte ist nahezu perfekt glatt. Im Zuge der Arbeit von Erichsen [56] konnten allerdings in Hochauflösungsbildern atomare Stufen entlang der Drähte entdeckt werden, wie es in Abbildung 4.1 (c) zu sehen ist. Die roten und grünen Pfeile symbolisieren hierauf eine Stufe nach oben bzw. nach unten. Neben den Stufen ist in 
Abbildung 4.1 (c) auch eine amorphe Kohlenstoff-Platin-Schicht mit einzelnen PlatinKristalliten zu erkennen. Diese Schicht umgibt den gesamten Draht und variiert in der Schichtdicke im Bereich von 5 bis $15 \mathrm{~nm}$, wobei die Dicke der Schicht zu den beiden Klebestellen hin zunimmt.

Die Drähte sind nach dem Transfer größtenteils defektfrei. Entlang der Drähte kann man aber vereinzelt kleine punktartige Kontraste ausmachen. Diese Kontraste sind in der Regel so klein, dass eine Analyse mit konventionellem TEM nicht möglich war, zumal die Kontraste erst bei der Bildanalyse entdeckt worden sind. Abbildung 4.2 (a) zeigt einen Draht nach dem Transfer mit drei dieser Kontraste, markiert durch die gestrichelten roten Kreise. (Man beachte, dass die Platinverdickung bewusst als Marker aufgebracht wurde.) In einem Fall konnten diese Punkte als kleine Versetzungsringe identifiziert werden (siehe (1) und (2) in Abbildung 4.2 (b)), so dass es sich möglicherweise bei den anderen Kontrasten ebenfalls um solche Defekte handelt.

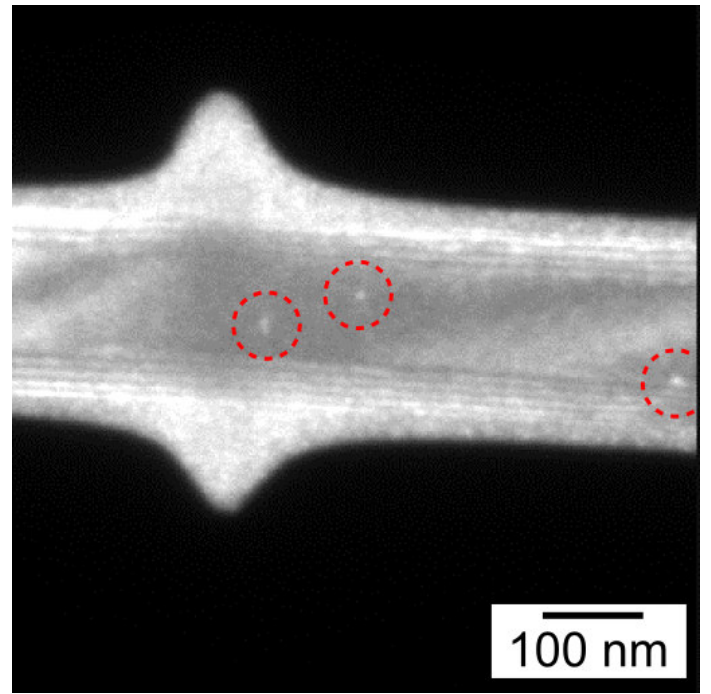

(a)

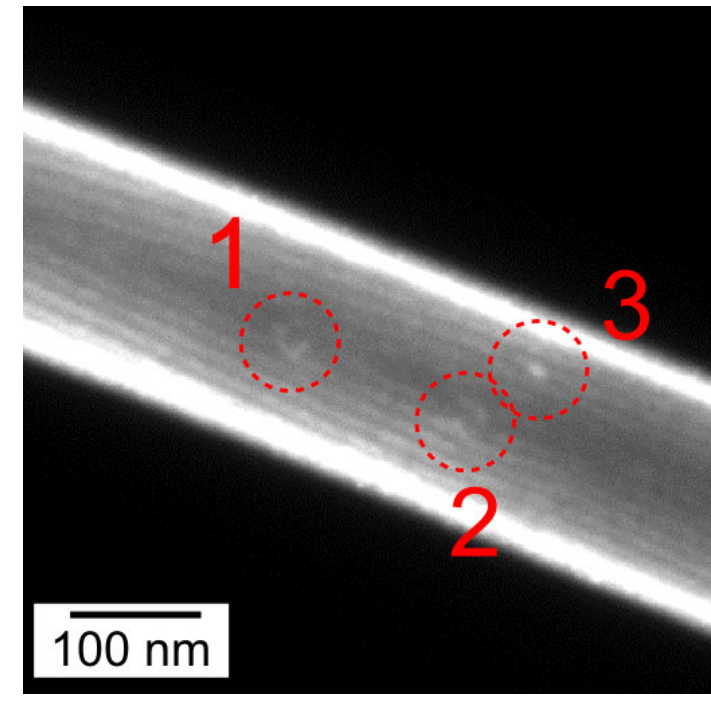

(b)

Abbildung 4.2.: Draht-Defekte vor dem Zugversuch in einkristallinen Drähten: (a) zeigt einen Draht nach dem Transfer mit drei punktartigen Kontrasten; (b) zeigt einen weiteren Draht mit punktartigen Defekten, wobei es sich bei (1) und (2) vermutlich um kleine Versetzungsringe handelt.

\subsubsection{Proben mit longitudinaler Zwillingsgrenze}

Die Proben mit longitudinaler Zwillingsgrenze stammen von dem selben Substrat wie die einkristallinen Proben. Da es sich bei den Zwillingsgrenzen um intrinsische Defekte 
handelt, ist eine Unterscheidung von einkristallinen Proben und verzwillingten Proben im REM nicht möglich. Eine eindeutige Identifizierung der Proben mit Zwillingsgrenze ist nur im TEM mit Hilfe einer Querschnittslamelle durchführbar.

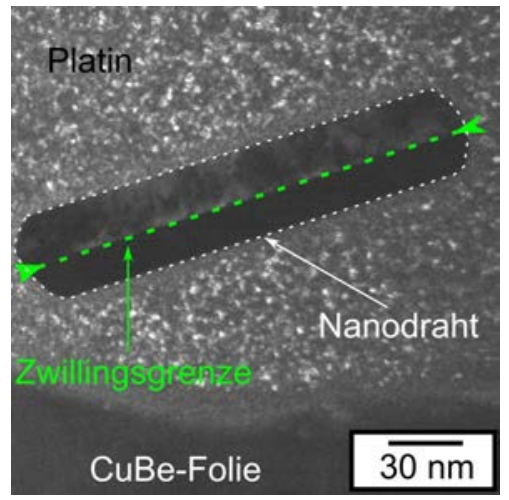

(a)

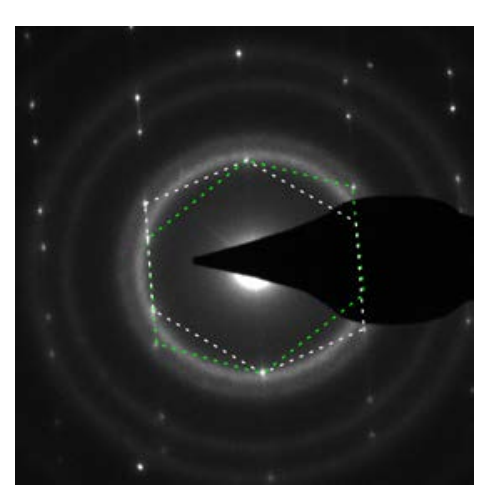

(b)

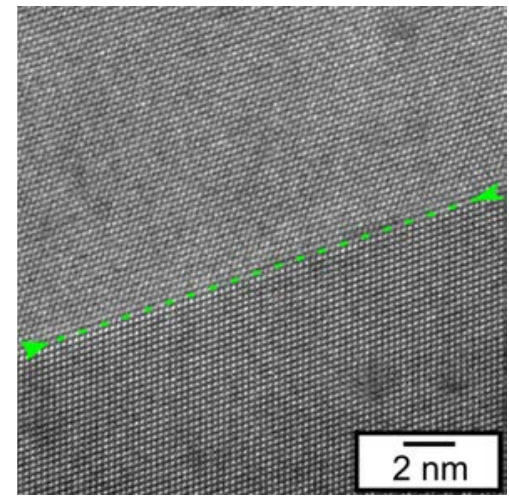

(c)

Abbildung 4.3.: Identifizierung von Zwillingsgrenzen im Draht: (a) zeigt die Dunkelfeldaufnahme einer Querschnittslamelle, die Zwillingsgrenze ist durch die gestrichelte grüne Linie markiert; (b) zeigt das Beugungsbild einer Querschnittslamelle, die Gitter der unterschiedlichen Zwillinge sind farblich markiert (grün und weiß); (c) zeigt das Hochauflösungsbild einer Zwillingsgrenze, die Zwillingsgrenze ist wieder durch die gestrichelte grüne Linie markiert.

In den Querschnittslamellen kann man die Zwillingsgrenzen in Dunkelfeldaufnahmen erkennen. Abbildung 4.3 zeigt eine solche Dunkelfeldaufnahme. Der Umriss des Drahtes ist mit einer gestrichelten weißen Linie markiert. Je nach ausgewähltem Beugungsreflex erscheint ein Teil des Drahtes als hell und der andere Teil als dunkel, getrennt durch die Zwillingsgrenze, die grün gestrichelt markiert ist. In Abbildung 4.3 (b) ist das Beugungsbild einer Lamelle von einem verzwillingten Draht zu sehen. Man kann deutlich die $\langle 110\rangle$-Orientierung der beiden Zwillingsparteien erkennen, die nochmal zusätzlich in grün und weiß gestrichelt hervorgehoben sind. In Abbildung 4.3 (c) ist eine Zwillingsgrenze in Hochauflösung zu sehen. Die Zwillingsgrenze ist wiederherum durch eine gestrichelte grüne Linie markiert. Sie läuft entlang einer $\{111\}$-Ebene. Weiterhin sind keine Stufen zu erkennen, so dass es sich um eine atomar glatte $\Sigma 3\{111\}$ Zwillingsgrenze handelt.

Insgesamt konnten 16 Proben auf diese Weise eindeutig als Drähte mit einer Zwillingsgrenze nachgewiesen werden. Neben Drähten mit einer einzelnen Zwillingsgrenze wie im Abbildung 4.3 ließen sich auch Drähte mit mehreren Zwillingsgrenzen iden- 
tifizieren, wobei die maximale Anzahl an Zwillingsgrenzen bei fünf lag. Der Großteil (12 von 16 Drähten) zeigt nur eine oder zwei Zwillingsgrenzen. In zwei Fällen konnten Zwillingsgrenzen auf zwei unterschiedlichen Scharen von $\{111\}$-Ebenen ausgemacht werden.

Weiterhin wurden 9 Proben ebenfalls als Drähte mit einer Zwillingsgrenze eingestuft, ohne für diese Drähte eine Querschnittslamelle herzustellen. (Die Gründe hierfür liegen zu einem darin, dass keine Teile des Drahtes mehr für eine Lamelle zur Verfügung standen, zum anderen im experimentellen Aufwand für die Präparation einer Lamelle). In diesen Fällen erfolgte die Einstufung durch das Auftreten einer Kontrastlinie in den TEM-Aufnahmen entlang der gesamten Drahtachse, wie sie auch bei Drähten beobachtet wurde, die später durch ein Lamelle eindeutig als Drähte mit einer Zwillingslamelle identifiziert wurden. Ein derartiger Kontrast ist beispielsweise in Abbildung $4.4 \mathrm{zu}$ erkennen.

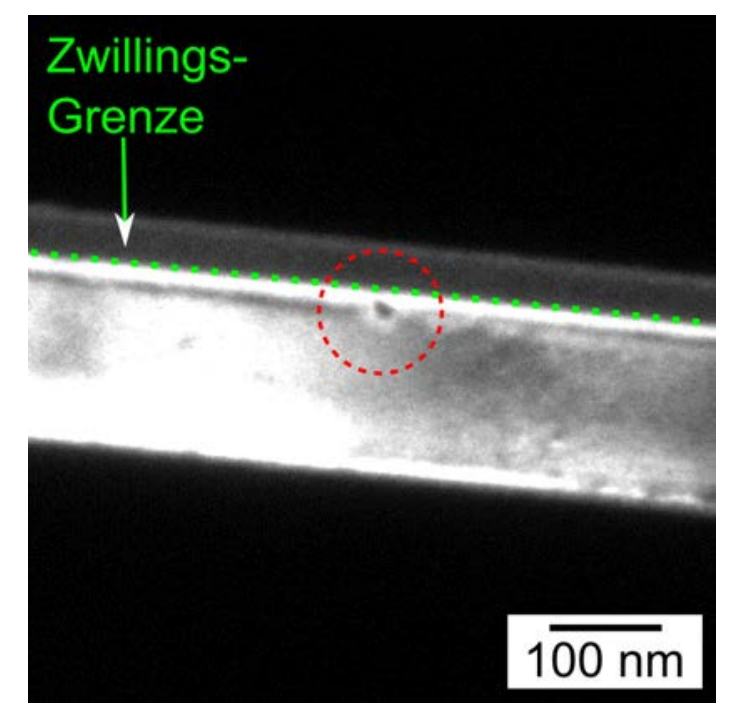

(a)

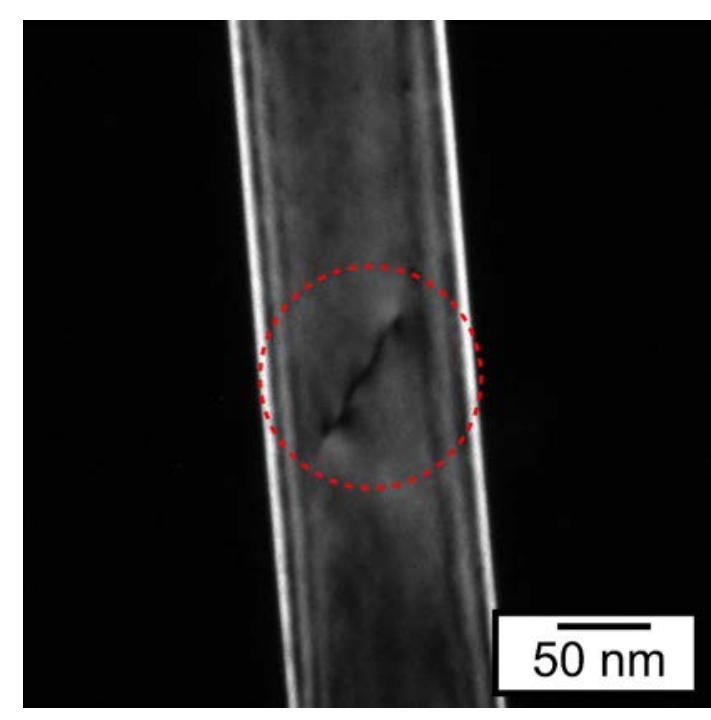

(b)

Abbildung 4.4.: Draht-Defekte vor dem Zugversuch in verzwillingten Drähten: (a) zeigt einen punktförmigen Defekt; (b) eine Versetzung

Die äquivalenten Durchmesser der Drähte variieren von $60 \mathrm{~nm}$ bis zu $180 \mathrm{~nm}$ und befinden sich damit im gleichen Größenbereich der einkristallinen Drähte. Die Höhen und Breiten der Drähte liegen zwischen 30nm und 360nm, wobei das Aspektverhältnis von Höhe zu Breite zwischen 1 und 8 liegt. Hierbei ist auffallend, dass ein großer Teil der verzwillingten Drähte (10 von 18 Drähten) ein Aspektverhältnis von größer als 3 aufweist und Drähte mit Zwillingsgrenzen somit dazu tendieren, eher bandartige 
Strukturen aufzuweisen. Die Zwillingsgrenzen liegen dabei üblicherweise parallel zur langen Kante des Drahtes.

Die Drähte mit Zwillingsstrukturen sind ebenso wie die einkristallinen Drähte größtenteils defektfrei. Allerdings lassen sich ebenso wie in den einkristallinen Drähten punktartige Kontraste entlang der Drähte finden wie in Abbildung 4.4 (a) zu sehen. Neben diesen punktartigen Kontrasten findet man gelegentlich eine Versetzung wie in Abbildung 4.4 (b) dargestellt. Diese Versetzungen sind entweder beim Wachstum entstanden oder im Zuge des Transferprozesses, da die Drähte nach der Identifizierung der Zwillingsgrenze mitunter ein zweites Mal transferiert worden sind und daher eine höhere Beanspruchung erfahren haben als die einkristallinen Drähte.

\subsection{Verformungsverhalten einkristalliner Drähte}

In diesem Abschnitt wird das Verformungsverhalten der einkristallinen Drähte erörtert. Hierzu konnten die Ergebnisse von 13 in-situ Zugversuchen im TEM und zusätzlich die Ergebnisse von 31 post-mortem Analysen von im REM getesteten Drähten herangezogen werden.

\subsubsection{Planare Defekte}

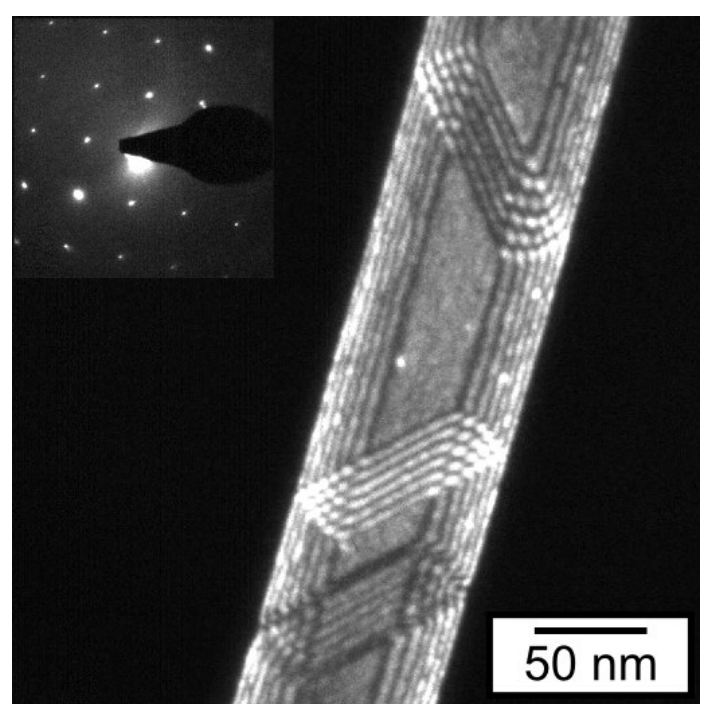

(a)

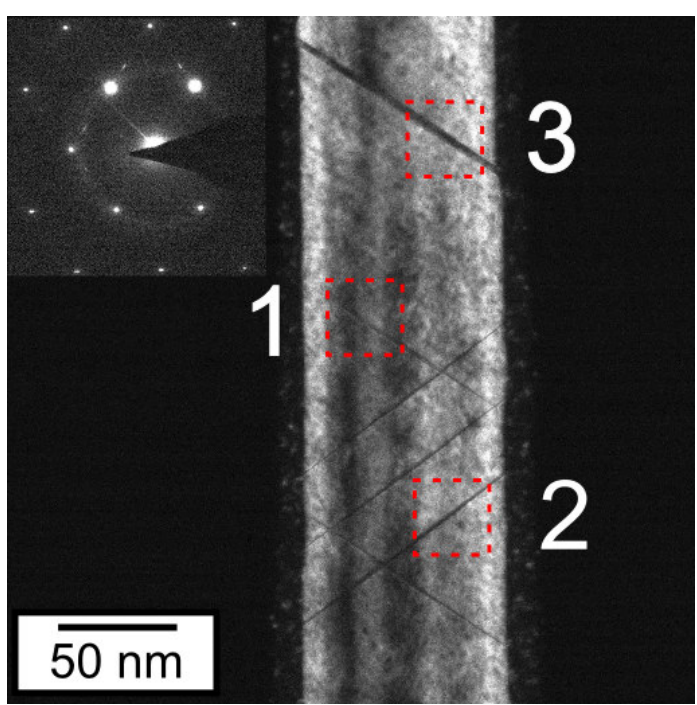

(b)

Abbildung 4.5.: TEM-Dunkelfeldaufnahmen von planaren Defekten: (a) (100)Zonenachse und (b) (110)-Zonenachse 
Planare Defekte sind die am häufigsten beobachteten Defekte in den einkristallinen Drähten, die im Zuge der Verformung entstanden sind. Sie konnten in allen 13 TEM-Versuchen während der Verformung sowie in 42 von 44 Proben nach der Verformung beobachtet werden. Abbildung 4.5 (a) zeigt eine Dunkelfeldaufnahme von drei planaren Defekten in der (100)-Zonenachse mit dem für planare Defekte typischen oszillierenden Kontrast. Man kann erkennen, dass die Defekte den gesamten Querschnitt des Drahtes durchkreuzen. In Abbildung 4.5 (b) ist ein weiteres Beispiel von planaren Defekten in der (110)-Zonenachse parallel zu den planaren Defekten erkennbar, die nun als dunkle Linien in genau zwei Orientierungen zur Drahtachse erscheinen. Ein Ausmessen der Winkel zwischen Kontrastlinie des planaren Defektes und Drahtachse ergibt $54^{\circ} \pm 1^{\circ}$ bzw. $54,5^{\circ} \pm 1^{\circ}$. und stimmt sehr gut mit dem theoretischen Winkel von 54,7 zwischen der (110)-Drahtachse und den beiden $\{111\}$-Ebenen überein, auf die die höchste Scherspannung wirkt.

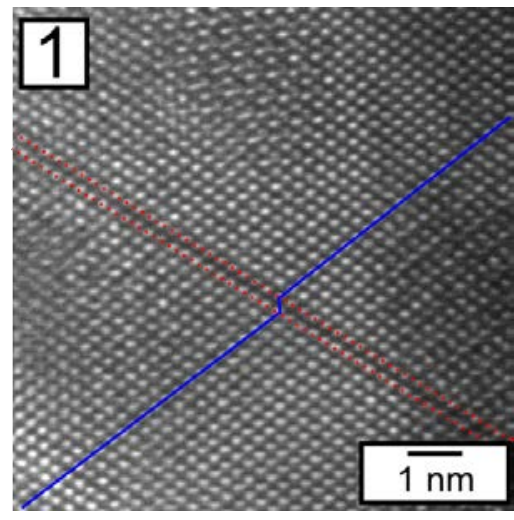

(a)

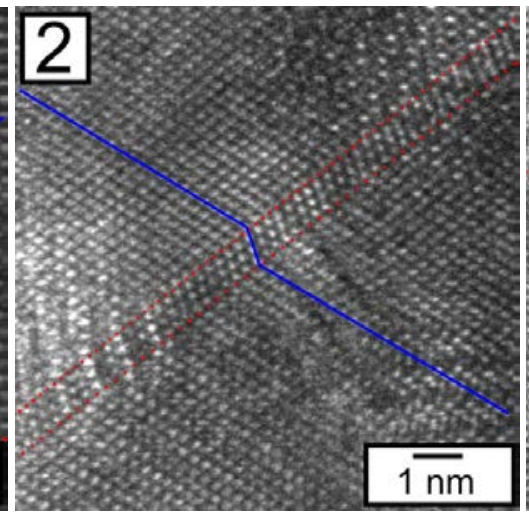

(b)

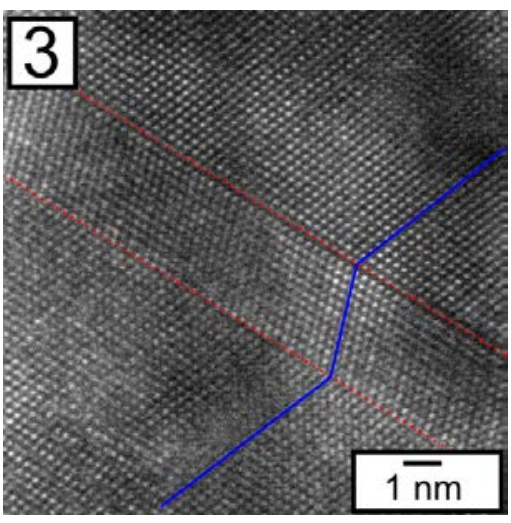

(c)

Abbildung 4.6.: Hochaufgelöste TEM-Bilder von planaren Defekten zeigen einen intrinsischen Stapelfehler (eine Atomlage) in (a), einen Zwilling mit drei Atomlagen in (b) und einen Zwilling mit 12 Atomlagen in (c)

Hochaufösung an diesen Defekten hat gezeigt, dass es sich bei den planaren Defekten entweder um Stapelfehler oder um kleine Nanozwillinge mit einer Breite von einigen Atomlagen handelt. In Abbildung 4.6 sind die drei markierten Bereiche aus Abbildung 4.5 (b) in Hochauflösung dargestellt. Die Grenzen der planaren Defekte sind jeweils mit einer rot gestrichelten Linie entlang der (111)-Ebene markiert, die blaue Linie entlang der zweiten (111)-Ebene dient zur Verdeutlichung der Verschiebung innerhalb des planares Defektes. In Abbildung 4.6 (1) ist ein planarer Defekt mit der Breite einer Atomlage zu sehen, so dass es sich um einen intrinsischen Stapelfehler handelt. In Ab- 
bildung 4.6 (2) ist ein planarer Defekt mit der Breite von 3 Atomlagen zu sehen, was der kleinsten Breite entspricht, ab der man von einem Nanozwilling sprechen kann. In Abbildung 4.6 (3) ist schließlich ein Zwilling mit einer Breite von 12 Atomlagen zu sehen. Man beachte in den Bildern, dass die Platin-Kohlenstoff-Schicht um die Drähte herum stellenweise zu einer Verzerrung der Bilder führen kann.

Neben den Hochaufösungsbildern bestätigt ebenfalls das Beugungsbild im Inset von Abbildung 4.5 (b) das Auftreten von Stapelfehlern und Zwillingen. Dort lässt sich zum einen ein schwaches Aufweiten der Beugungsreflexe entlang der (111)-Richtungen, ein sogenanntes 'Streaking', beobachten, was durch die Präsenz von Stapelfehlern hervorgerufen wird. Zum anderen kann man zusätzliche Beugungsreflexe durch die Nanozwillinge ausmachen.

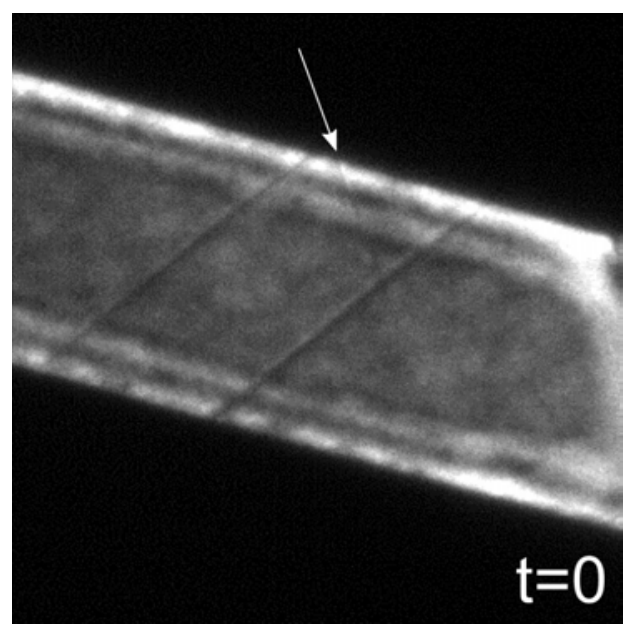

(a)

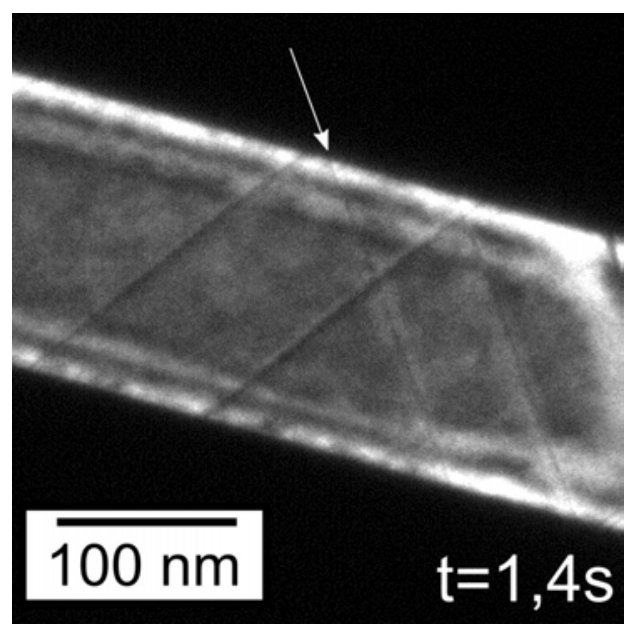

(b)

Abbildung 4.7.: Hindernis für Defekte: (a) Der weiße Pfeil markiert einen planaren Defekt, der zunächst nur bis zu einem planaren Defekt auf dem anderen (111)-System verläuft; (b) zeigt den planaren Defekt nach dem kompletten Durchlaufen des Drahtes 1,4s später

Während der Verformung ist zu erkennen, wie planare Defekte nach und nach entlang des Drahtes auftauchen. Typischerweise erscheinen die planaren Defekte innerhalb eines Kameraframes, was einer Zeitspanne von 100ms entspricht. Wie in Abbildung 4.5 ersichtlich kann es dabei auch zum Kreuzen von zwei planaren Defekten der zwei möglichen $\{111\}$-Ebenen kommen. Üblicherweise ist beim Entstehen des zweiten Defektes keine Verzögerung feststellbar, so dass ein planarer Defekt in den meisten Fällen scheinbar keinen Einfluss auf die Bewegung eines Defektes auf der konjugierten Ebene hat. 
In einigen Fällen konnte weiterhin beobachtet werden, dass ein planarer Defekt zunächst nur bis zu einem planaren Defekt auf der zweiten $\{111\}$-Ebene verläuft und erst nach einer gewissen Zeit den gesamten Draht durchquert. Ein solches Beispiel ist in Abbildung 4.7 dargestellt, wobei zwischen Abbildung 4.7 (a) und (b) 1,4s liegen. Ein planarer Defekt kann sich in einigen Fällen doch als ein Hindernis für die Bewegung von Defekten herausstellen. Im späteren Verlauf der Verformung kann es dazu kommen, dass mehrere planare Defekte von einem planaren Defekt auf der konjugierten Ebene aufgehalten werden.

\section{Wachsen von planaren Defekten}

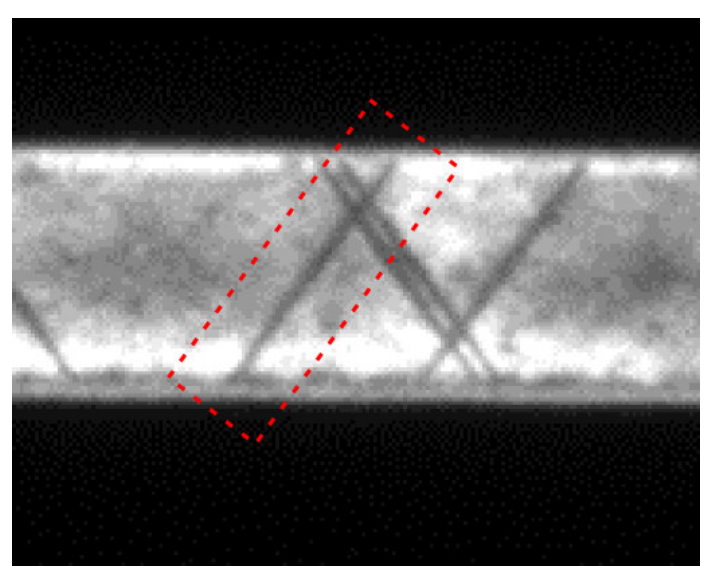

(a)

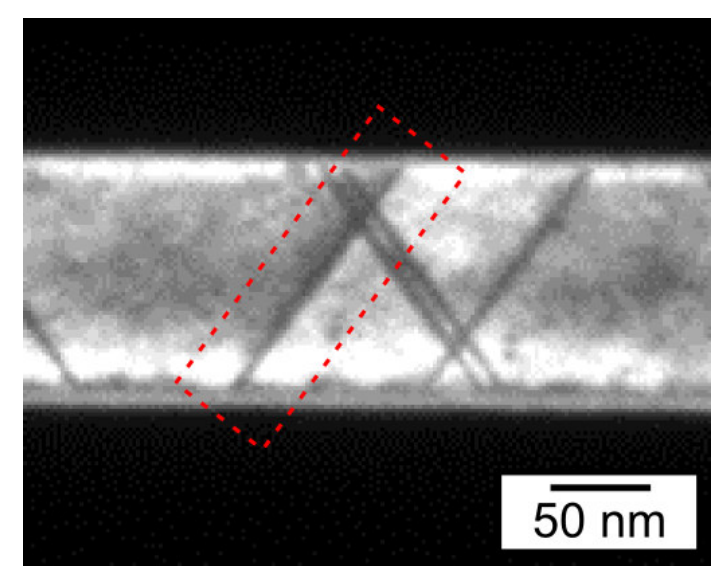

(b)

Abbildung 4.8.: Kontrastwechsel an einem planaren Defekt (durch rot gestrichelte Box markiert): a) vor dem Kontrastwechsel; b) nach dem Kontrastwechsel

Neben dem Auftauchen von neuen planaren Defekten konnte ebenfalls eine Verbreiterung von bereits entstandenen planaren Defekten und damit das Wachsen von Zwillingen beobachtet werden. Das wurde dadurch deutlich, dass sich der Kontrast bei bereits vorhandenen planaren Defekten schlagartig änderte. Abbildung 4.8 zeigt einen planaren Defekt vor (a) und nach (b) einem Kontrastwechsel. Der Kontrastwechsel erfolgt innerhalb eines Kameraframes, was auf eine Nukleation ähnlich dem Auftauchen von neuen planaren Defekten schließen lässt. Die einfachste Erklärung für einen solchen Kontrastwechsel ist die Verbreiterung des planaren Defektes um eine oder mehrere Ebenen. Ein solches Verhalten ließ sich in insgesamt neun Proben während der Verformung feststellen. 


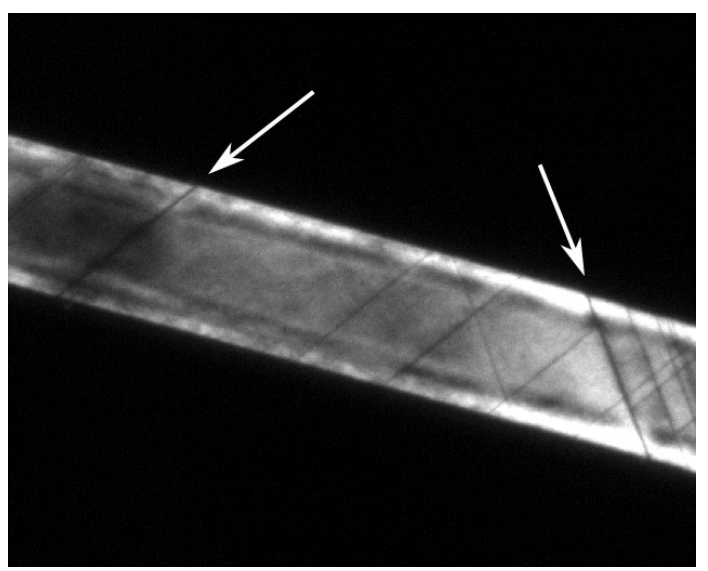

(a)

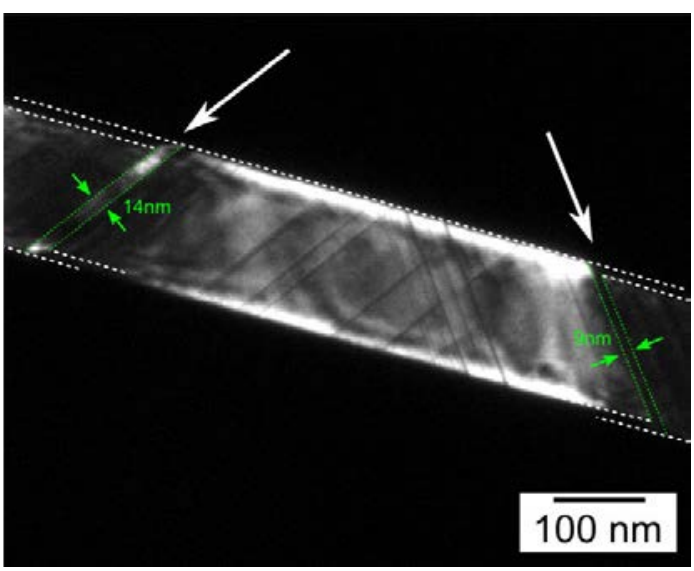

(b)

Abbildung 4.9.: Wachstum von planaren Defekten: (a) zeigt zwei planare Defekte jeweils markiert durch einen weißen Pfeil gegen Anfang der Verformung, (b) zeigt die beiden gleichen planaren Defekte gegen Ende der Verformung mit einer Breite von 14nm bzw. 9nm

In zwei Drähten war zudem direkt eine Verbreiterung der planaren Defekte auf eine Breite von mehr als 5nm im Zuge der Verformung erkennbar. Hierbei kam es nach dem Auftauchen des planaren Defektes nach und nach zu einer Verbreiterung wie in Abbildung $4.9 \mathrm{zu}$ sehen ist. In Abbildung 4.9 (a) sind mit einem weißen Pfeil zwei planare Defekte gegen Anfang der Verformung zu sehen. Abbildung 4.9 (b) zeigt die beiden gleichen Defekte gegen Ende der Verformung. Die beiden Defekte weisen nun eine Breite von 14nm bzw. 9nm auf. Einhergehend mit Zwillingen einer solchen Breite ist ebenfalls eine Formänderung des Drahtes, wie durch die gestrichelte weiße Linie angedeutet.

Wie in Abbildung 4.9 deutlich erkennbar ist, können Zwillinge auf beiden $\{111\}$ Ebenen vorkommen, so dass das Wachsen von planaren Defekten nicht nur auf eine der beiden $\{111\}$-Ebenen beschränkt ist. Weiterhin kann man in Abbildung 4.9 erkennen, dass es neben der Verbreiterung der beiden Zwillinge ebenfalls zum Auftauchen von neuen planaren Defekten sowohl in unmittelbarer Nähe als auch entlang des Drahtes kommt. Das Wachsen eines Zwillings scheint folglich nicht präferiert, sondern gleichberechtigt gegenüber der Nukleation von neuen Defekten zu sein. 


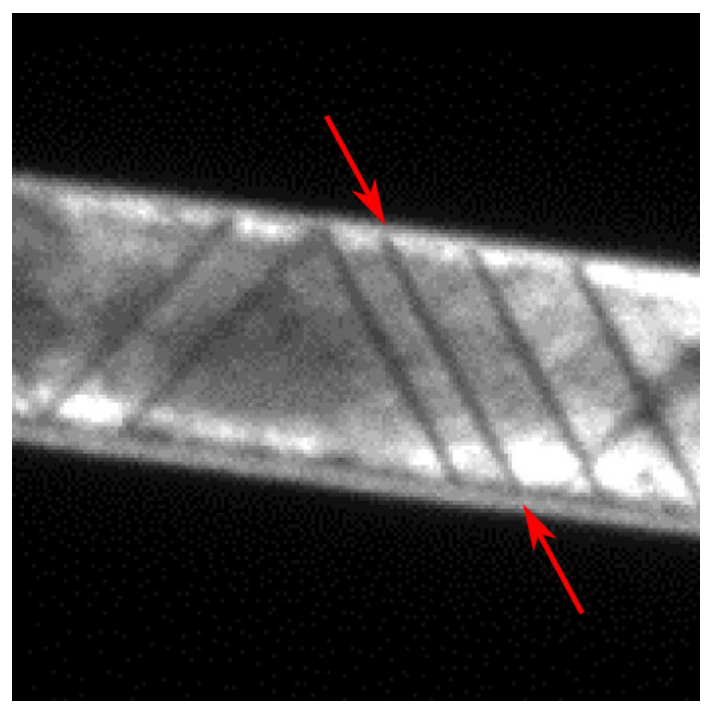

(a)

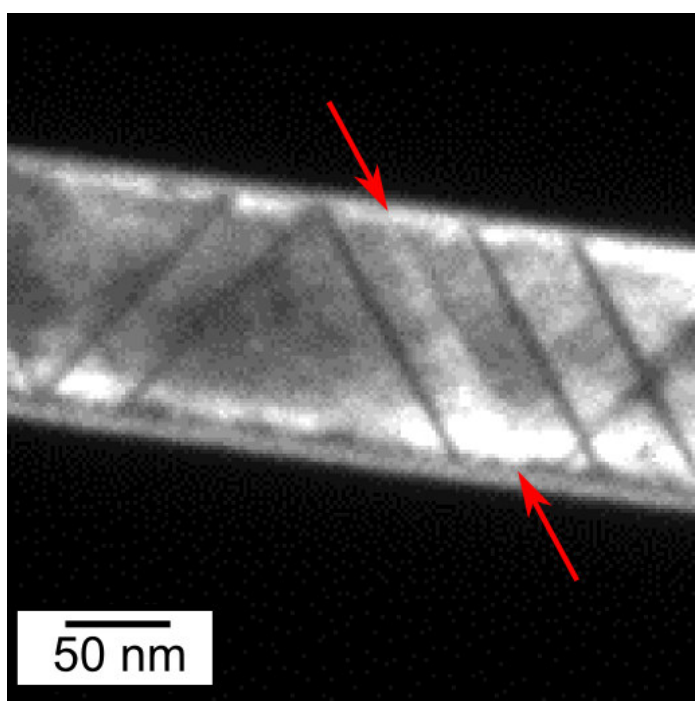

(b)

Abbildung 4.10.: Verschwinden von einzelnen planaren Defekten: Einzelbilder aus dem Video bei einem Zugversuch in der (110)-Zonenachse vor (a) und nach (b) dem Verschwinden eines einzelnen planaren Defekts (markiert durch rote Pfeile)

\section{Verschwinden von planaren Defekten}

Neben der Verbreiterung können planare Defekte mit zunehmender Verformung auch wieder verschwinden. Dabei kann es vorkommen, dass planare Defekte einzeln oder kollektiv verschwinden. Abbildung 4.10 zeigt zwei Einzelbilder aus einem Zugversuch vor (a) und nach (b) dem Verschwinden eines einzelnen planaren Defektes, jeweils markiert durch zwei rote Pfeile. Nach dem Verschwinden des planaren Defektes in Abbildung 4.10 (b) kann man entlang der \{111\}-Ebene, auf der der planare Defekt lag, weiterhin einen schwachen Kontrast erkennen. Hierbei kann es sich möglicherweise um eine Oberflächenstufe handeln, die auf das Durchlaufen einer vollen Versetzung hindeuten würde.

In Abbildung 4.11 ist die Sequenz aus drei TEM-Dunkelfeldaufnahmen zusammen mit einer Skizze zur Veranschaulichung der Anordnung der planaren Defekte dargestellt. Abbildung 4.11 (a) zeigt die Ausgangssituation mit einer Reihe von planaren Defekten auf beiden $\{111\}$-Ebenen. Man beachte, dass einige planare Defekte nicht den gesamten Draht durchlaufen, sondern an einem planaren Defekte des zweiten $\{111\}$ Systems enden, wie auch schon in Abbildung $4.7 \mathrm{zu}$ sehen ist, so dass es auch hier $\mathrm{zu}$ einer Wechselwirkung zwischen Defekten der beiden $\{111\}$-Systeme gekommen ist. 

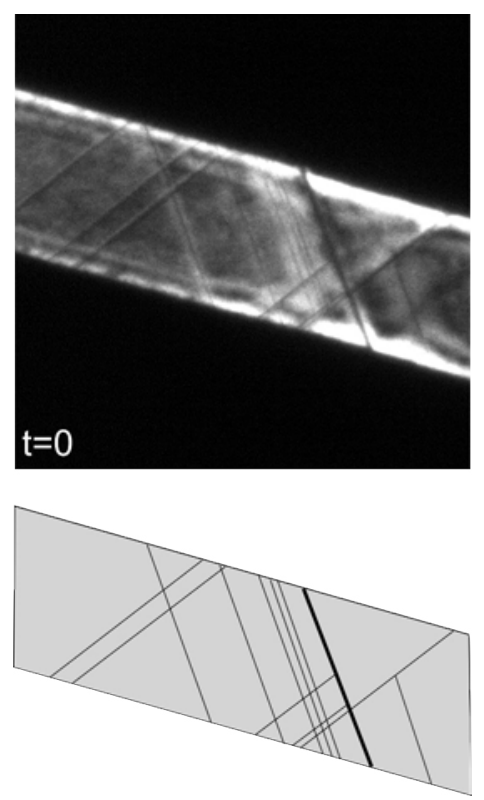

(a)
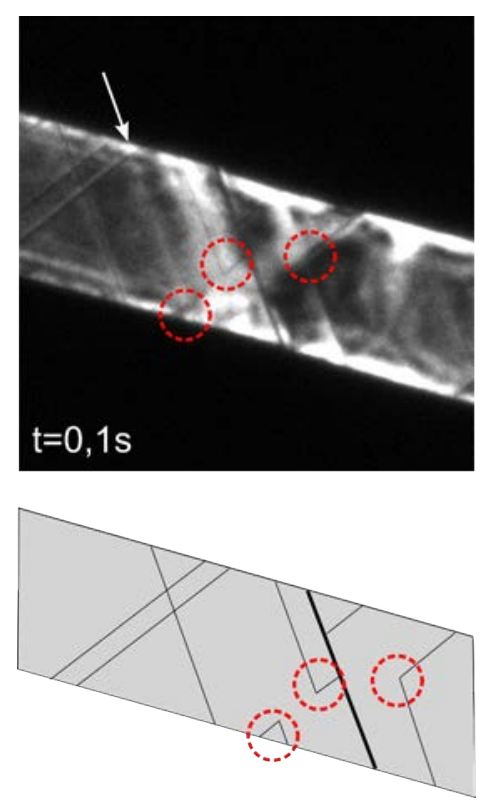

(b)
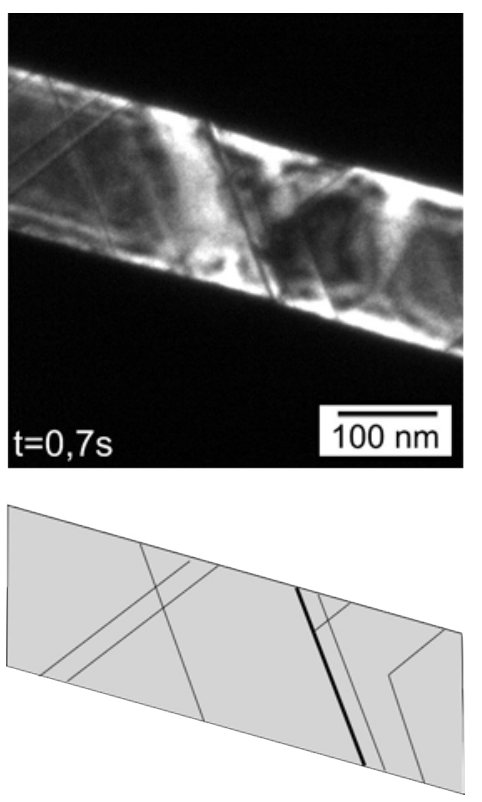

(c)

Abbildung 4.11.: Verschwinden planarer Defekte: $\mathrm{Zu}$ sehen sind jeweils TEMDunkelfeldaufnahmen und ein Skizze zur Verdeutlichung der Anordnung der planaren Defekte.(a) zeigt die Ausgangslage vor dem Verschwinden der planaren Defekte; (b) zeigt den Draht 100ms später nach dem Verschwinden von planaren Defekten, wobei in einigen Fällen die Kombination planarer Defekte auf beiden \{111\}-Ebenen zurückbleiben, die schließlich in (c) ebenfalls verschwinden. 
Abbildung 4.11 (b) zeigt den Draht 100ms später. Einige planare Defekte sind nicht mehr vorhanden, wohingegen in 3 Fällen die planaren Defekte nur partiell verschwunden sind. Dabei entsteht ein Konstrukt aus planaren Defekten beider unterschiedlichen $\{111\}$-Ebenen, so dass beide planaren Defekte scheinbar an dem planaren Defekt des zweiten $\{111\}$-Systems enden. Diese Stellen sind in Abbildung 4.11 (b) mit einem rot gestrichelten Kreis markiert. Mit einem weißen Pfeil markiert ist wiederum eine $\{111\}$-Ebene, auf der zuvor ein planarer Defekte beobachtet wurde und nun nur ein schwacher Kontrast verbleibt, wie bereits in Abbildung 4.10 beobachtet wurde. In Abbildung 4.11 (c) verschwinden schließlich zwei der Konstrukte aus planaren Defekten der beiden $\{111\}$-Ebenen.

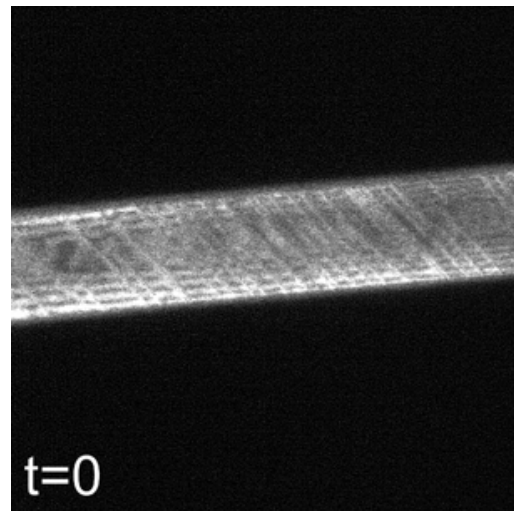

(a)

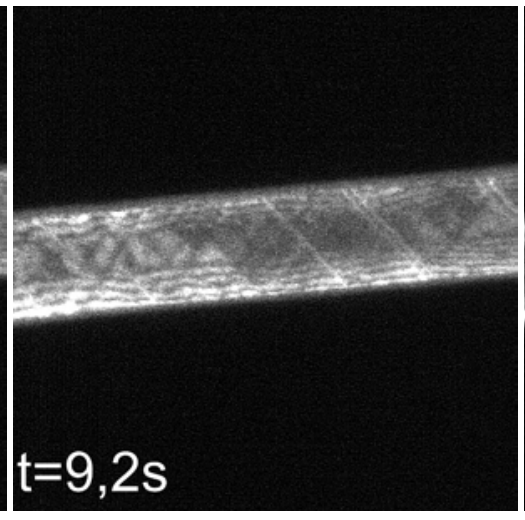

(b)

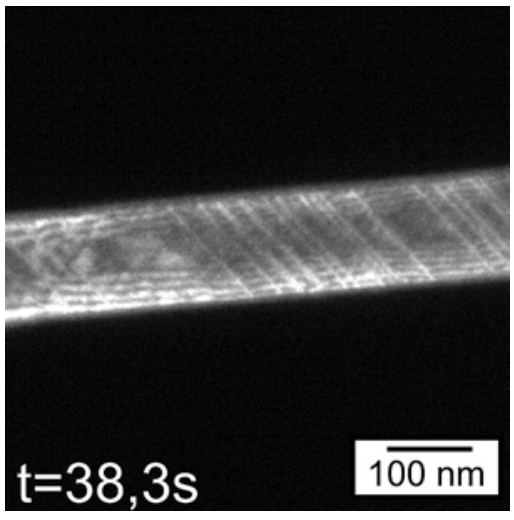

(c)

Abbildung 4.12.: Kollektives Verschwinden planarer Defekte: Im Zuge der Verformung verringert sich die Anzahl planarer Defekte auf einer der beiden (111)Ebenen von 23 in (a) durch kollektives Verschwinden auf 6 planare Defekte in (b). Mit zunehmender Verformung erscheinen neue planare Defekte im gleichen Bereich und die Anzahl steigt auf 17.

Ein solch kollektives Verschwinden konnte auch in ganzen Bereichen des Drahtes nachgewiesen werden. Üblicherweise ließ sich ein solch kollektives Verschwinden in Bereichen mit einer hohen Anzahl an planaren Defekten auf beiden \{111\}-Systemen erkennen. Nach dem Verschwinden können in dem gleichen Bereich des Drahtes neue planare Defekte erscheinen. Abbildung 4.12 zeigt ein solches Beispiel. Man beachte, dass durch die Abbildebedingungen lediglich planare Defekte auf einem der beiden \{111\}-Systeme deutlich als helle Linien erkennbar sind. Schwache dunkle Kontraste deuten aber auf die Präsenz planarer Defekte auf dem zweiten $\{111\}$-System hin. Im Zuge der Verformung verringert sich die Anzahl von sichtbaren planaren Defekten von 
23 in Abbildung 4.12 (a) auf 6 planare Defekte in (b). Mit zunehmender Verformung erscheinen neue planare Defekte im gleichen Bereich und die Anzahl steigt auf 17 planare Defekte in Abbildung 4.12 (c).

\begin{tabular}{c|c} 
Drahtbezeichnung & $\begin{array}{c}\text { Anzahl planare Defekte } \\
\text { pro Mikrometer }\end{array}$ \\
\hline TEMDis6 & $>100$ \\
Normal2 & $\approx 15$ \\
Normal3 & $\approx 45$ \\
Normal6 & $>70$ \\
\hline
\end{tabular}

Tabelle 4.1.: Verschwinden planarer Defekte während Verformung

Das Verschwinden planarer Defekte war in 10 Drähten während der Verformung beobachtbar. Um dieses Verhalten quantifizieren zu können, wurde jedes Verschwinden eines planaren Defektes im Verlauf eines Videos gezählt und mit der im Bildausschnitt befindlichen Länge des Drahtes eine Dichte (Anzahl planarer Defekte pro Mikrometer) bestimmt. Für 6 Drähte war es möglich, auf diese Weise deren Dichte zu bestimmen. Für die übrigen Drähte war eine Quantifizierung nicht möglich. Durch Überlagerung von Kontrasten und sehr eng beieinander liegender planaren Defekten stellt dieser Wert jeweils eine untere Abschätzung dar. Die Werte sind in Tabelle 4.1 zu sehen und reichen von circa 15 planaren Defekten pro Mikrometer bis über 100 pro Mikrometer.

\subsubsection{Einfluss von anfänglichen Defekten}

Wie in Abschnitt 4.1 beschrieben findet man entlang der Drähte punktartige Kontraste. Während der Verformung ist zu erkennen, wie die ersten planaren Defekte scheinbar bevorzugt an den punktförmigen Kontrasten entstehen. Ein solches Beispiel ist in Abbildung 4.13 gezeigt. Abbildung 4.13 (a) zeigt das Einzelbild aus einem Video während eines Zugversuchs direkt vor dem ersten Nukleationsevent in der (111)-Zonenachse. Der vorhandene Defekt ist durch eine rot gestrichelten Kreis markiert. Abbildung 4.13 (b) zeigt den Draht 100ms später. Man kann einen planaren Defekt erkennen, der sich durch den gesamten Drahtquerschnitt erstreckt. Hierbei ist zu beachten, dass für den gezeigten Draht der Querschnitt ein sehr hohes Aspektverhältnis hat und der planare Defekt dadurch nahezu als Linie in der (111)-Zonenachse erscheint. Der Ort des vorher vorhandenen Defekts ist weiterhin durch den rot gestrichelten Kreis markiert. Es lässt sich allerdings nicht feststellen, ob der punktartige Defekt noch vorhanden ist. 


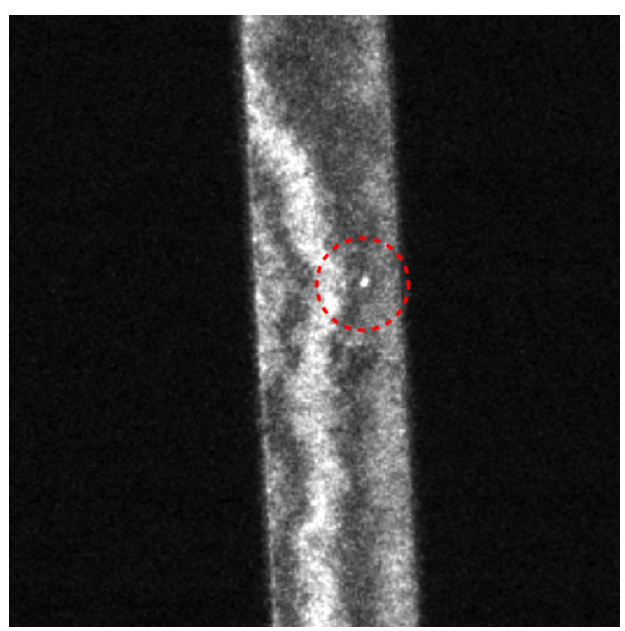

(a)

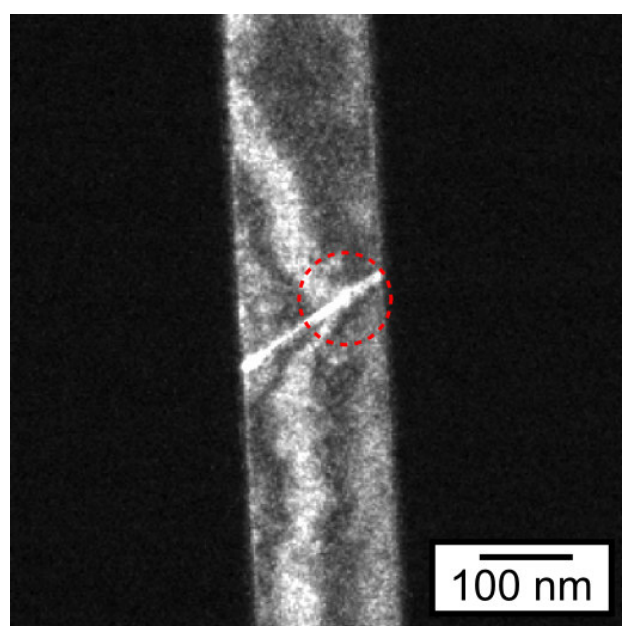

(b)

Abbildung 4.13.: Beginn der plastischen Verformung: (a) zeigt den Draht vor dem ersten Nukleationsevent; rot markiert ist ein Defekt im Draht, (b) zeigt den ersten planaren Defekt, wobei in rot weiterhin die Stelle des Defektes markiert ist

Das Auftauchen der ersten planaren Defekte über bereits vorhandenen Defekten ließ sich in drei Drähten direkt im Video beobachten. In einem weiteren Draht lagen starke Hinweise auf ein solches Verhalten vor. Die vorhandenen Defekte scheinen folglich die Nukleation von neuen Defekten zu begünstigen und erst im Anschluss kommt es zur Nukleation von weiteren Defekten entlang der gesamten Drahtlänge. Da innerhalb der TEM-Versuchsreihe keine Dehnungs- oder Spannungsinformationen vorhanden sind, lässt sich nur schwer abschätzen, wie viel früher die Nukleation an den Defekten abläuft. Das Auftauchen weiterer planarer Defekte entlang des Drahtes relativ zeitnah lässt jedoch auf kleine Unterschiede in der Spannung bzw. Dehnung zwischen der Nukleation an einem Defekt und der Nukleation ohne einen solchen Defekt schließen. Gleichzeitig lässt sich folgern, dass der Defekt nach dem Auftauchen des ersten planaren Defektes scheinbar kein bevorzugter Punkt für weitere Nukleationen von Defekten ist. Darüber hinaus bleibt festzustellen, dass nicht jeder vorhandene punktartige Defekt zu einem Nukleationsevent führt.

Um den Einfluss von Defekten auf das Verformungsverhalten weitergehend zu untersuchen, wurden in 5 einkristalline Proben Defekte eingebracht, wie in Abschnitt 3.4 beschrieben. Diese Beanspruchung vor einem Zugversuch führt üblicherweise zum Einbringen sowohl von Versetzungen an dem Punkt der maximalen Beanspruchung 


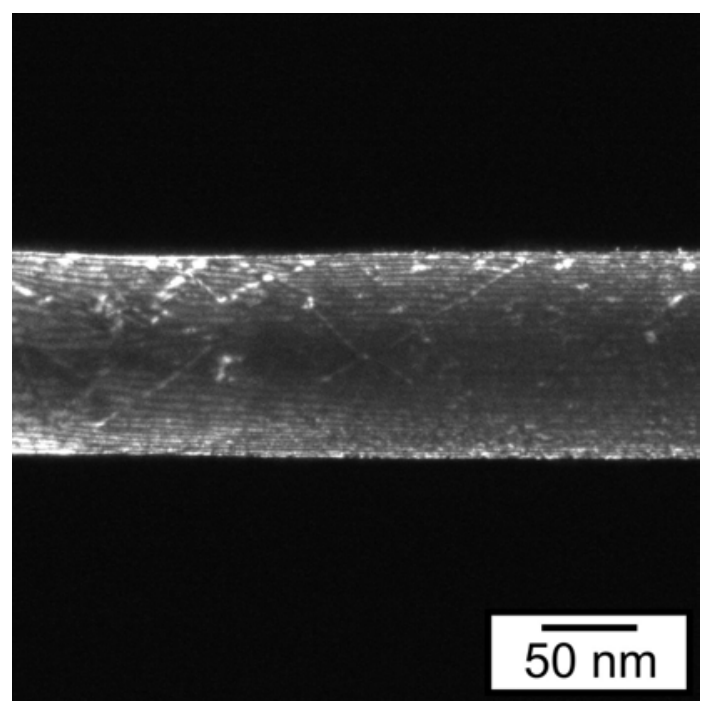

(a)

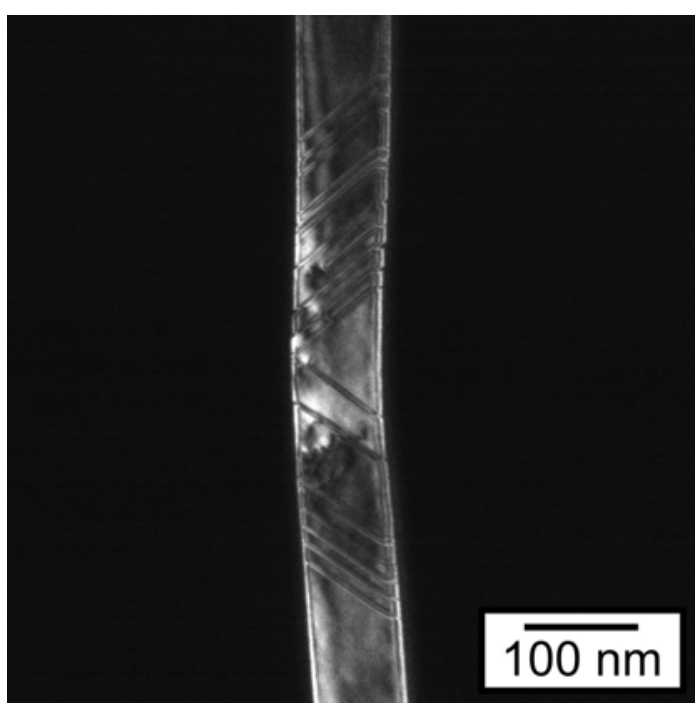

(b)

Abbildung 4.14.: Einbringen von Defekten vor dem Versuch: Typischerweise findet man an dem Punkt der maximalen Beanspruchung volle Versetzung (a). Neben den Versetzungen treten häufig auch planare Defekte entlang des Drahtes auf (b). Weiterhin kann man üblicherweise eine kleine Delle bzw. Verjüngung in der Drahtoberfläche erkennen.

als auch zu einigen planaren Defekten entlang des Drahtes wie in Abbildung $4.14 \mathrm{zu}$ sehen ist. Weiterhin ist üblicherweise eine kleine Delle bzw. Verjüngung an der Stelle zu erkennen, an der der 'Indenter' den Draht getroffen hat.

Abbildung 4.15 (a) zeigt einen beanspruchten Draht zu Beginn eines Zugversuchs. Man erkennt in der Mitte des Bildes eine Mischung aus hellen und dunklen Kontrasten, die durch die Versetzungen hervorgerufen werden. Ebenfalls ist eine Verjüngung des Drahtes erkennbar. Im rechten Teil der Abbildung kann man weiterhin drei planare Defekte ausmachen. Diese drei Defekte verbleiben während der Anfangsdeformation an Ort und Stelle und können so zur Orientierung in der Abfolge von Abbildung 4.15 verwendet werden.

Die ersten Kontrastwechsel während des Zugversuchs können bei den vorher eingebrachten Versetzungen beobachtet werden. Dieses war in vier der fünf vorher beanspruchten Drähte der Fall. Die Kontrastwechsel bei den Versetzungen können zum einen sehr ruckartig vonstatten gehen, aber auch teilweise eher kontinuierlich ablaufen. In drei Fällen ließ sich bei den Kontrastwechseln eine Art Bewegung oder Umorientierung der Versetzungen beobachten. Dabei scheinen sich die Kontraste zunächst auf bestimmte kristallographische Ebenen zur Oberfläche der Drähte zu orientieren, wie es 


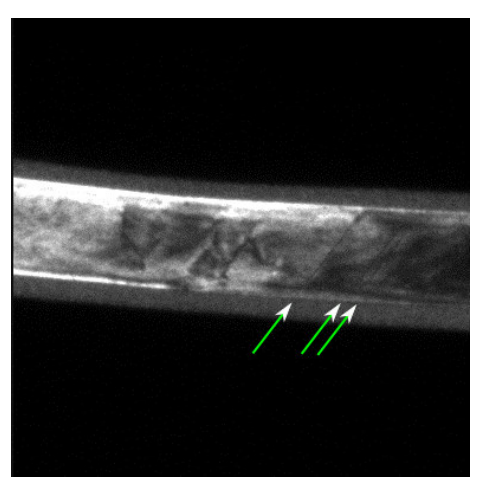

(a)

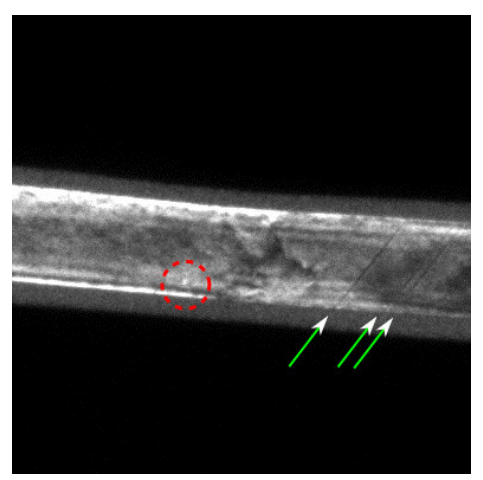

(d)

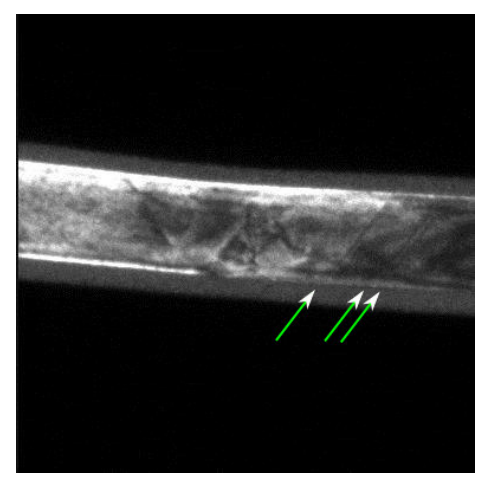

(b)

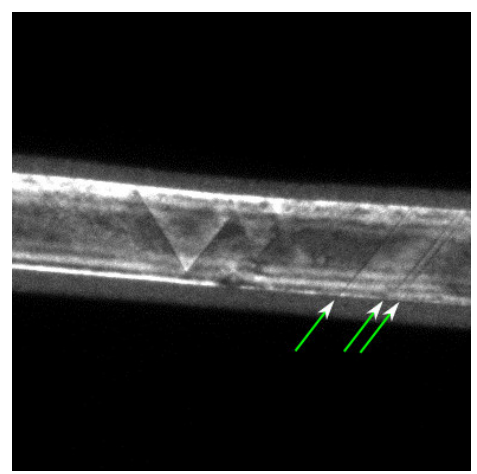

(e)

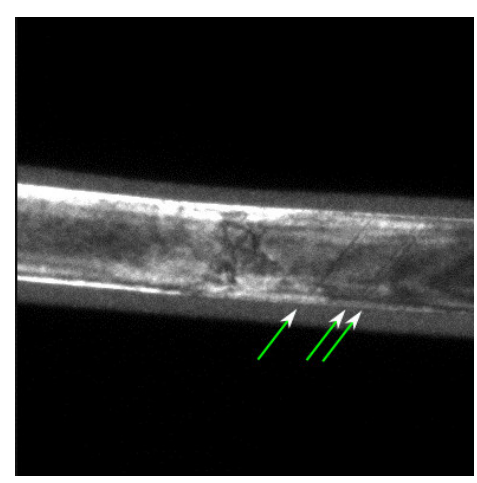

(c)

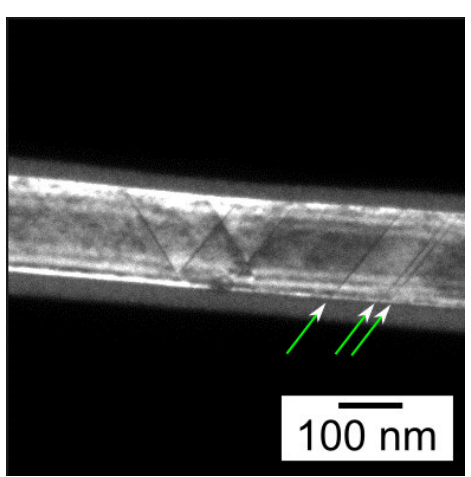

(f)

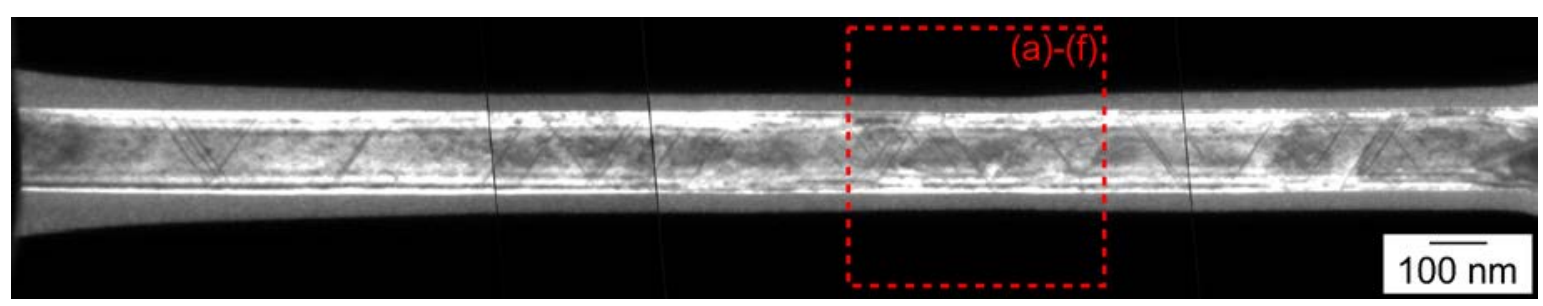

(g)

Abbildung 4.15.: Verformung in einem Draht mit eingebrachten Versetzungen: (a)-(f) zeigt die Entwicklung zu Beginn der plastischen Verformung. Die Versetzungen verlassen nach und nach den Draht. (g) zeigt eine Übersicht im späteren Verlauf der Verformung mit einer Verteilung von planaren Defekten entlang der gesamten Drahtlänge. 
z.B. in Abbildung 4.15 (b) der Fall ist. Anschließend können die Versetzungen den Draht scheinbar durch die Oberfläche verlassen. Es können hierbei sehr schwache Kontraste im Draht oder an der Oberfläche zurückbleiben. Mit der Zeit nehmen die ursprünglichen Kontraste immer weiter ab, bis schließlich in Abbildung 4.15 (e) kaum Kontraste der Versetzungen zurückbleiben.

Im Verlauf der Dehnung entstehen die ersten planaren Defekte direkt an dem Ort, an dem vorher die Kontraste der Versetzungen zu beobachten waren. Dabei können die planaren Defekte zum einen aus hellen Punktdefekten (markiert durch einen rot gestrichelten Kreis) entstehen. So kann man in Abbildung 4.15 von (d) nach (e) sehen, wie sich insgesamt 2 scharfe Kontrastlinien aus einem Punktdefekt gebildet haben. Hierbei entwickelt sich zunächst einer der beiden Kontraste aus dem Punktdefekt heraus, anschließend der zweite. Der Punktdefekt liegt in dieser Projektion nicht an der Oberfläche des Drahtes und dementsprechend durchlaufen die Kontraste auch nicht den gesamten Draht. Weiterhin können sich direkt an den alten Versetzungen planare Defekte bilden. Das Entstehen von planaren Defekten aus bzw. an Kontrasten der ursprünglichen Defekte war in 4 Fällen direkt beobachtbar. Die weitere Verformung erfolgte dann analog zu den Proben ohne vorherige Beanspruchung. So zeigt 4.15 (g) den Draht im späteren Verlauf der Verformung mit einer Verteilung von planaren Defekten entlang des gesamten Drahtes. Mit dem gestrichelten roten Quadrat ist der Bereich aus den Bilden (a)-(f) markiert. Versetzungen sind nicht mehr zu erkennen. Damit scheinen die vorher eingebrachten Versetzungen nicht zu einem Wechsel im Verformungsmechanimus geführt zu haben. Weiterhin ist auffällig, dass die durch die Vorbehandlung vorhandenen planaren Defekte nicht als besonderer Nukleationspunkt für weitere Defekte dienen. Stattdessen können auch diese planaren Defekte im Zuge der Verformung wieder verschwinden. So sind die drei planaren Defekte in Abbildung 4.15 (g) nicht mehr vorhanden. Ein derartiges Verhalten konnte in sämtlichen Proben beobachtet werden, in denen durch die Beanspruchung planare Defekte eingebracht worden sind.

\subsubsection{Verhalten bei Entlastung}

Um zu sehen, wie sich die entstandenen Defekte beim Entlasten verhalten, wurde bei zwei Drähten (AuNormal3 und AuH4) nach dem Erscheinen von einigen Defekten die Bewegungsrichtung des Zughalters umgekehrt und die beiden Aufnahmepunkte wurden wieder zusammengefahren. 
Im Fall von AuNormal3 wurde die Bewegung analog zu Belastung schrittweise per Hand zurückgenommen. Hierbei befand sich der gleiche Drahtabschnitt während der gesamten Entlastung im sichtbaren Bereich der Kamera. Abbildung 4.16 (a) zeigt den Draht zu Beginn der Entlastung und (b) den Draht am Ende der Entlastung.

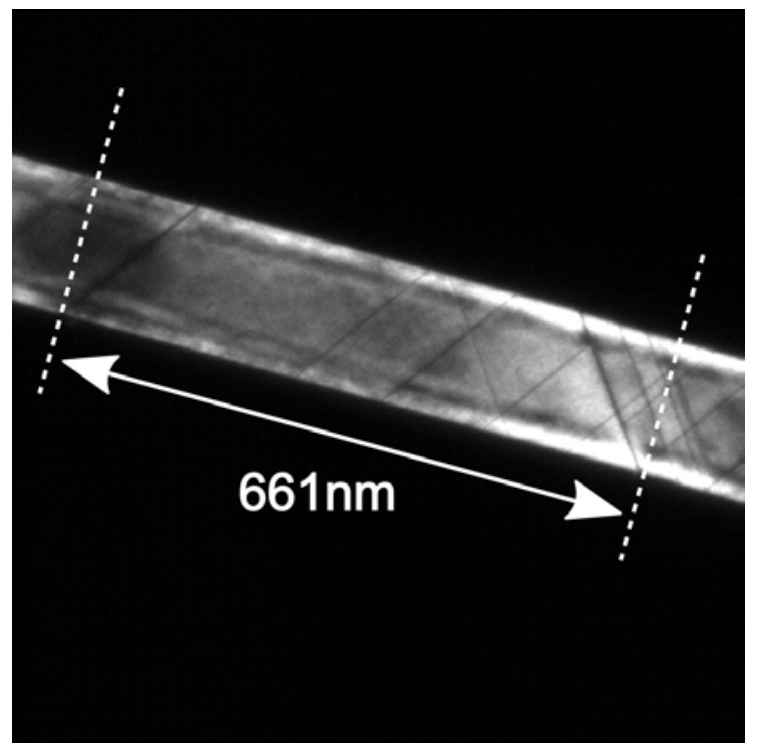

(a)

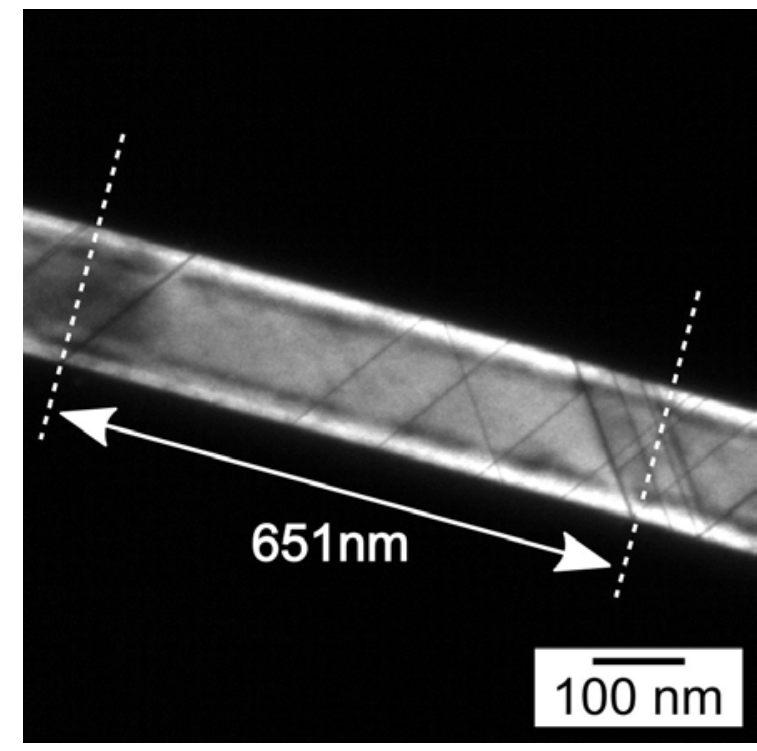

(b)

Abbildung 4.16.: Entlastung bei einem einkristallinen Draht: (a) zeigt den Draht zu Beginn der Entlastung, (b) zeigt den Draht am Ende der Entlastung.

Zu Beginn der Entlastung in Abbildung 4.16 (a) sind insgesamt 16 planare Defekte erkennbar. Zum Abschätzen der Dehnung während der Entlastung wurden der Abstand zwischen den beiden breiteren Defekten verwendet, der in Abbildung 4.16 jeweils durch die gestrichelten weißen Linien markiert ist. Zu Beginn der Entlastung beträgt der Abstand 661nm.

Während der Entlastung lassen sich keine Veränderungen an den Kontrasten der Defekte feststellen. Insbesondere ist kein Verschwinden von Kontrasten zu beobachten, so dass die Anzahl an Defekten am Ende der Entlastung in Abbildung 4.16 (b) weiterhin 16 beträgt. Der Abstand zwischen beiden gewählten Markern beträgt nun $651 \mathrm{~nm}$, entsprechend einer Entlastung von 10nm. Der Draht wurde also insgesamt um $\Delta \epsilon=10 \mathrm{~nm} / 661 \mathrm{~nm} \approx 1,5 \%$ entlastet. Inwieweit der Draht sich am Ende der Entlastung noch unter Zugspannung befindet, lässt sich jedoch nicht erfassen. Ein Biegen des Drahtes, wie es beim Überschreiten des Übergangs von Zug- auf Druckspannung zu erwarten wäre, ließ sich jedoch nicht wahrnehmen. 
Bei der Entlastung des zweiten Drahtes wurde die 'auto-zero'-Funktion des Halters verwendet. Dabei kam es zu Beginn der Entlastung zu einer starken lateralen Bewegung, wodurch der Draht während der Entlastung nicht zu beobachten war. Deshalb konnte eine Quantifizierung über die entlastete Dehnung nicht erfolgen. Da der Halter jedoch komplett zusammengefahren wurde und damit weiter als im Fall von AuNormal3, ist anzunehmen, dass eine vergleichbare oder größere Entlastung vorgelegen hat. Ein Vergleich aus Bildern vor und nach der Entlastung zeigt keine Veränderung in der Defektstruktur, wobei hier jedoch nur ein kleiner Bereich von 600nm Breite verwendet werden konnte.

\subsubsection{Bruchmorphologie und Bruchverhalten}

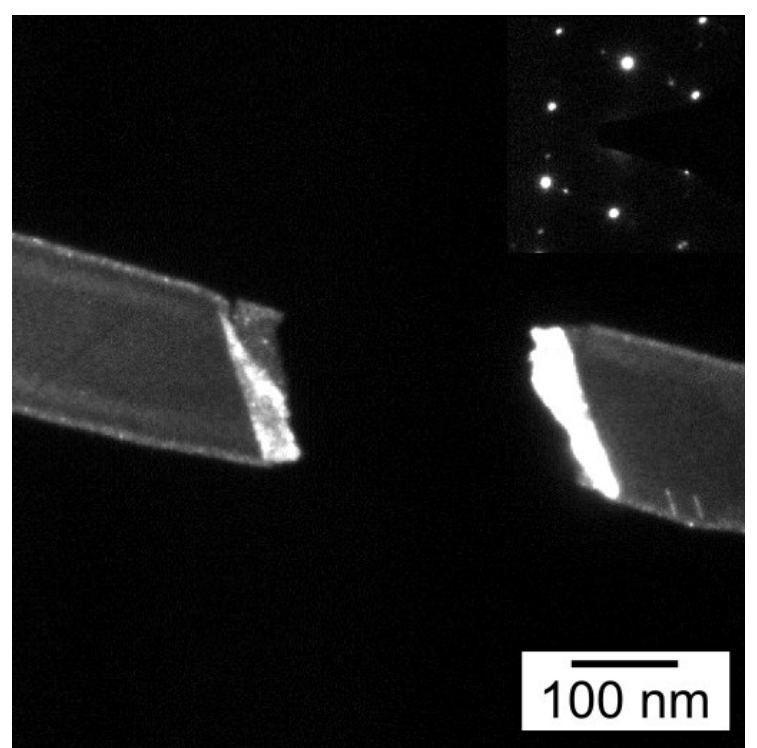

(a)

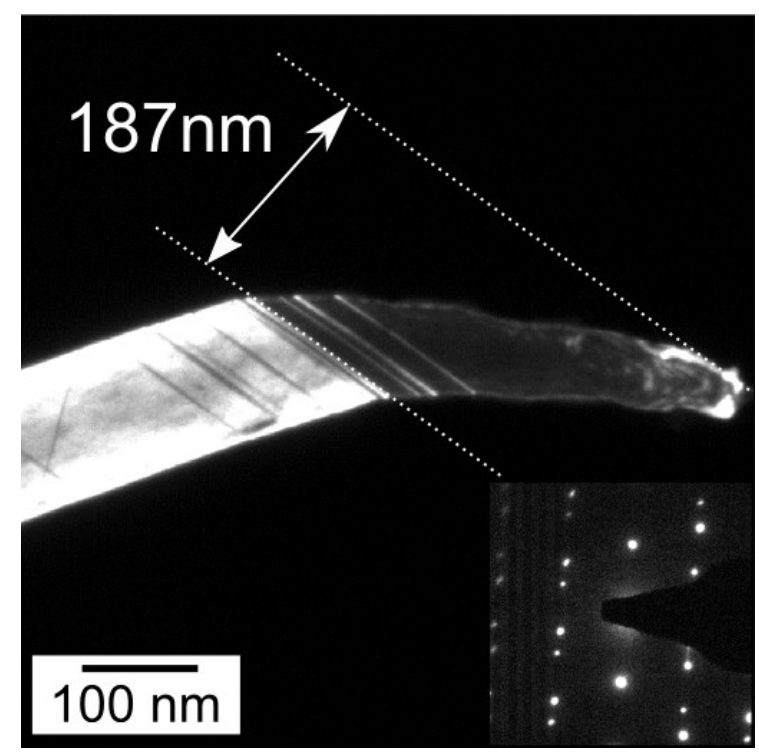

(b)

Abbildung 4.17.: Zwillinge an der Bruchkante: a) Zwillingsbereiche an beiden Seiten der Bruchkante; b) Bruchkante mit einem 187nm breiten Zwilling

An der Bruchkante weisen die meisten Drähte (32/36) einen verzwillingten Bereich auf. Dabei findet man gewöhnlich auf beiden Seiten der Bruchkante einen verzwillingten Bereich, wie in Abbildung 4.17 a) zu sehen ist. Ein Versagen der Drähte findet also typischerweise innerhalb des verzwillingten Bereichs statt.

Die Breite der Zwillinge an der Bruchkante variiert von von $28 \mathrm{~nm}$ bis zu $187 \mathrm{~nm}$. Letzteres ist in Abbildung 4.17 b) zu sehen. Das Beugungsbild im Inset zeigt eindeutig die Verzwilligung durch das Auftreten der zusätzlichen Beugungsreflexe. Zur Bildge- 
bung wurde jedoch ein Reflex der ursprünglichen Orientierung verwendet, so dass der Zwilling dunkel im Vergleich zum hellen Draht erscheint. Man beachte, dass innerhalb des Zwillings einige Ebenen hell erscheinen und damit zur ursprünglichen Orientierung des Drahtes gehören. Damit scheint ein Wachsen des Zwillings nicht ausschließlich auf benachbarten Ebenen stattzufinden. Weitere große Zwillinge entlang des Drahtes scheinen eher die Ausnahme zu sein. So konnten Zwillinge mit einer Breite von mehr als 5nm lediglich in 10 von 42 Proben erkannt werden. Zusätzlich ist der Zwilling an der Bruchkante typischerweise deutlich größer als sämtliche Zwillinge entlang des Drahtes. Lediglich zwei Drähte zeigen auch entlang ihrer Länge eine ausgeprägte Verzwilligung. So scheint zum einen in einem Großteil der Drähte ein Zwilling ab einer bestimmten Größe zum Versagen des Drahtes zu führen. Zum anderen scheint es im Zuge des Reißens zu einem schnellen Wachsen dieses Zwillings zu kommen.

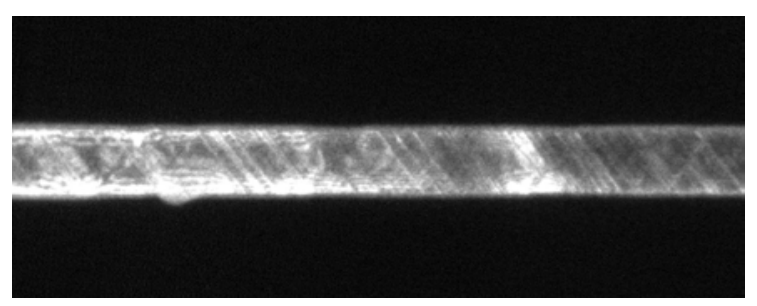

(a)

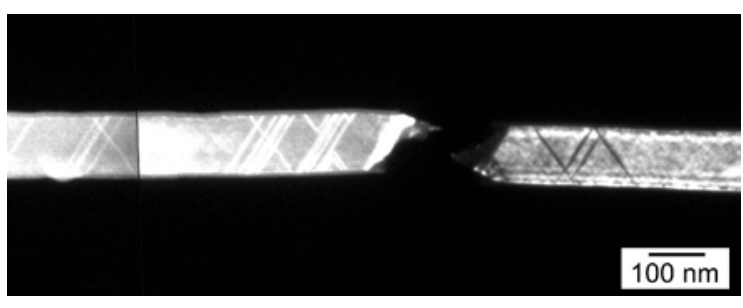

(b)

Abbildung 4.18.: Abruptes Bruchverhalten: (a) zeigt einen Draht ein Frame vor dem Versagen, (b) zeigt den Draht aus drei Einzelbildern zusammengesetzt nach dem Bruch

Dies wird ebenfalls durch das beobachtete Bruchverhalten in den in-situ TEMVersuchen bestätigt. In 7 Fällen konnte nach vorheriger homogener Verformung entlang des Drahtes ein abruptes Ausbilden eines größeren Zwillings festgestellt werden, wobei es in 4 Fällen direkt zum Versagen des Drahtes innerhalb des Zwillings kam. In den übrigen drei Fällen bildet sich zunächst ein stark verjüngter Bereich innerhalb des Zwillings, wonach durch Fortsetzung der Dehnung der Draht ebenfalls versagt. Im Fall von AuDisTEM6 lag der Ort des Versagens direkt im Kameraausschnitt. So zeigt Abbildung 4.18 den Draht direkt vor dem Reißen (a) sowie aus drei Einzelbildern zusammengesetzt nach dem Reißen (b). Man beachte, dass die Abbildebedingungen zwischen den beiden Bruchkanten unterschiedlich sind, so dass die Defekte im linken Teil hell erscheinen und im rechten Teil dunkel. An der Bruchkante ist ein Zwilling mit einer Breite von insgesamt 55nm zu erkennen, der sich im Zuge des Reißens gebildet 
haben muss, da vorher kein großer Zwilling im Draht vorhanden war. In einem anderen Draht kann man vorher schon einen Zwilling mit einer Breite von wenigen Nanometern erkennen.

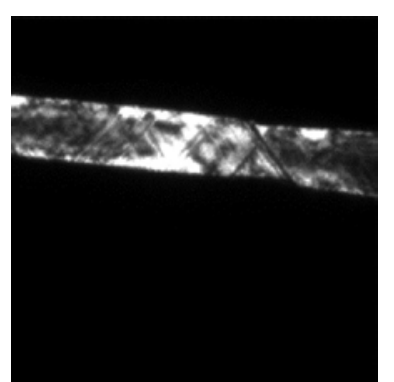

(a)

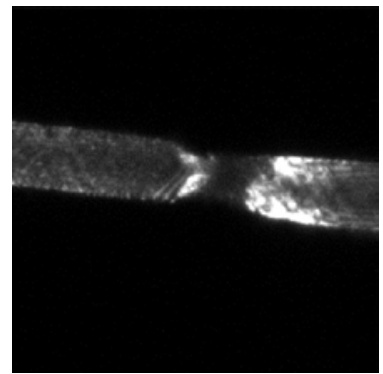

(b)

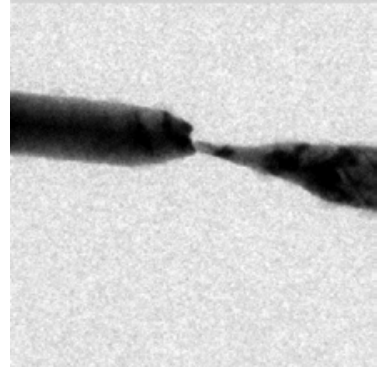

(c)

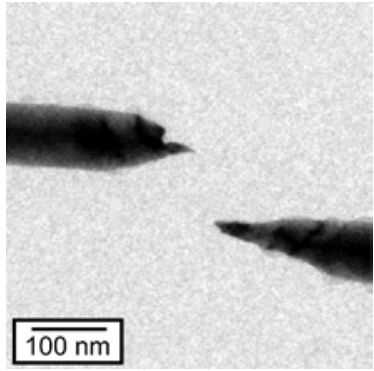

(d)

Abbildung 4.19.: Kontinuierliches Bruchverhalten: a)- d) Sequenz einer kontinuierlichen Verjüngung unter der Ausbildung einer filementartigen Stuktur

In drei Drähten ließ sich dagegen ein anderes Bruchverhalten feststellen. Hier kam es nicht zu einem abrupten Ausbilden eines Zwillings, sondern stattdessen über einen längeren Zeitraum zu einer kontinuierlichen Verjüngung in der Weise, dass in einer Art fließender Verformung die Ausbildung einer sehr dünnen filamentartigen Struktur vor dem endgültigen Versagen stattfand. Die Sequenz eines solchen Verhaltens ist in Abbildung $4.19 \mathrm{zu}$ sehen, wobei es sich in (a) und (b) um Dunkelfeld- und in (c) und (d) um Hellfeld-Aufnahmen handelt. Ausgangspunkt kann dabei ein Zwilling mit einer Breite von wenigen Nanometern gewesen sein, wie in (a) zu sehen.

Neben dem schnellen Wachstums eines Zwillings kommt es im Zuge des Reißens auch zum Verschwinden von planaren Defekten entlang des Drahtes. So zeigt Abbildung 4.20 einen Draht einen Kameraframe vor dem Reißen (oben) und die gleiche Stelle nach dem Reißen (unten). Vor dem Versagen konnten 52 planare Defekte in dem Bildausschnitt gezählt werden. Nach dem Versagen befinden sich dagegen nur noch 8 planare Defekte im gleichen Bereich, so dass circa 85\% der planaren Defekte im Zuge des Reißens verschwunden sind.

Das Verschwinden von planaren Defekten ließ sich insgesamt bei 6 Drähten feststellen. In vier Fällen war es möglich, die Anzahl der verschwundenen Defekte zu quantifizieren. So lag der Anteil an verschwundenen planaren Defekten zwischen $40 \%$ und dem Beispiel in Abbildung 4.20 von 85\%. Dabei scheinen besonders Defekte zu verschwinden, die vorher durch einen planaren Defekt auf der konjugierten Ebene am Durchlaufen gehindert wurden oder im Bereich des Drahtes mit einer hohen Dichte 


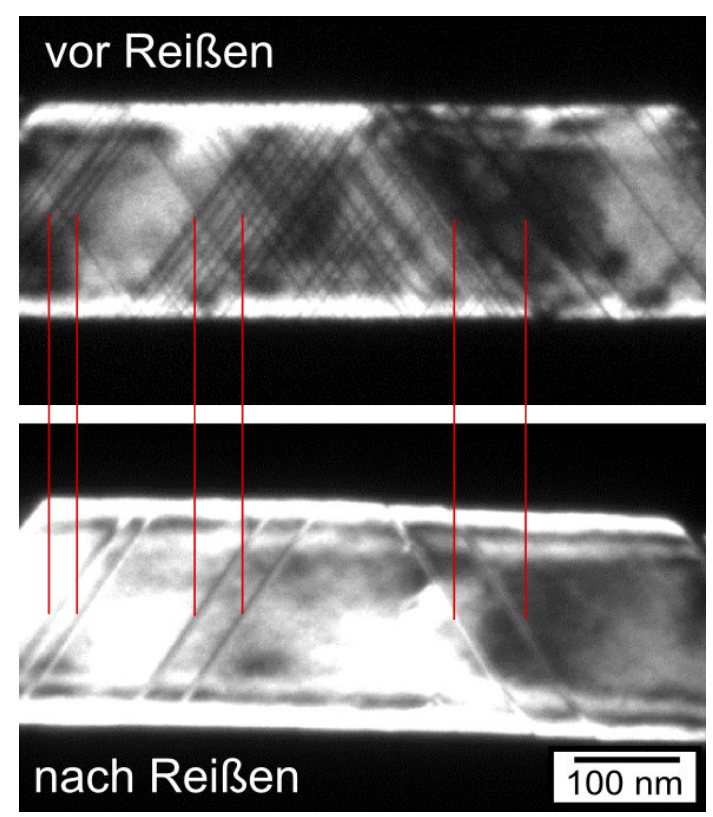

Abbildung 4.20.: Verschwinden von planaren Defekten beim Versagen des Drahtes

von planaren Defekten auf beiden Ebenen zu finden waren. In einem Draht konnte beobachtet werden, dass nur diejenigen planaren Defekte verschwinden, die vor dem Versagen nicht komplett durch den Draht verliefen. Es scheint also eine Reaktion der Defekte auf das Wegfallen der äußeren Spannung beim Versagen der Drähte in der Weise zu geben, dass die Defekte dabei wieder aus dem Draht herausgetrieben werden.

\section{Speicherung der Defekte}

Trotz der Tatsache, dass planare Defekte im Zuge des Reißens wieder verschwinden können, kann die gespeicherte Anzahl an planaren Defekten nach dem Reißen Aufschluss über das Verformungsverhalten geben. Um dies genauer zu bestimmen wurden sämtliche planaren Defekte entlang der Drähte gezählt bzw. vermessen. Für jeden planaren Defekt, der im konventionellen TEM mit einer Breite von unter 5nm erscheint, wurde eine Breite von 4,7 Atomlagen angenommen, was einem Mittelwert von 11 hochaufgelösten planaren Defekten aus diesem Größenbereich entspricht. Da diese Aufnahmen bei dem Draht aufgenommen wurden, der die größte plastische Dehnung erfahren hat, stellt der Wert von 4,7 eine Obergrenze dar. Für alle großen Zwillinge erfolgte eine Ausmessung einzeln in den TEM-Aufnahmen, wobei ein Fehler von 2 Pixeln auf beiden Seite des Zwillings angenommen wurde, was etwa 5nm entspricht. Mit dem Ebenenabstand von Gold zwischen den $\{111\}$-Ebenen von 0,235nm lässt sich dann die Anzahl 
der Ebenen pro Zwilling bestimmen.

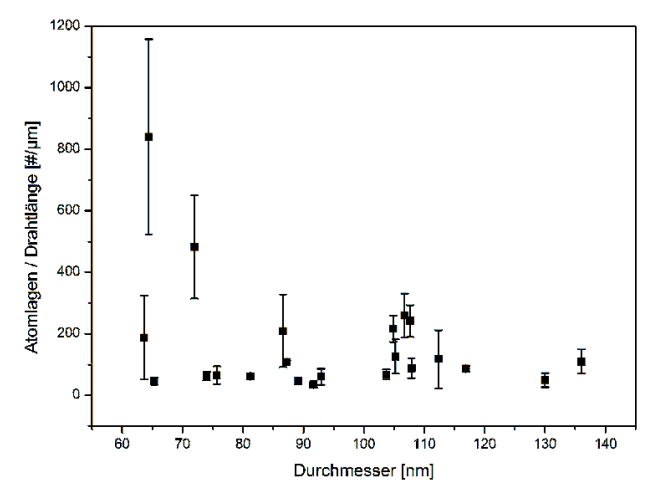

(a)

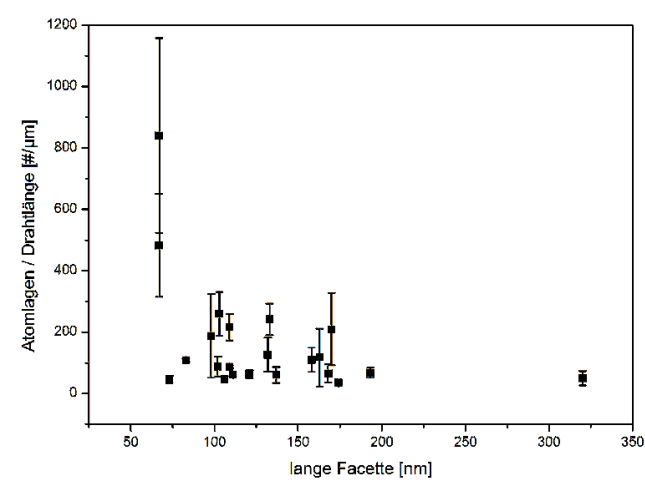

(c)

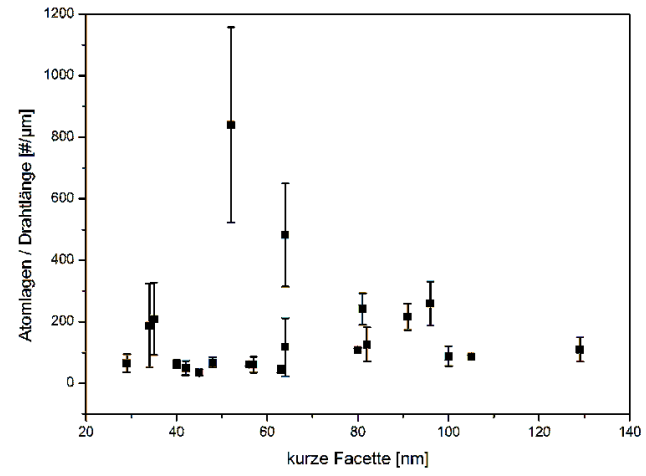

(b)

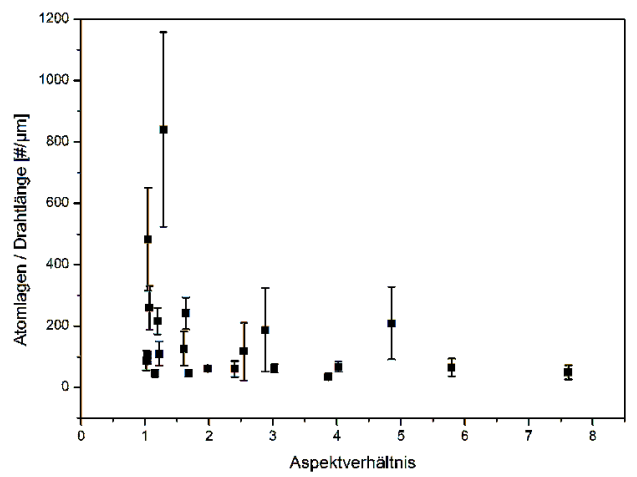

(d)

Abbildung 4.21.: Gespeicherte Anzahl von Atomlagen normiert auf die jeweilige Drahtlänge gegen den äquivalenten Durchmesser (a), die kurze (b) und lange (c) Facette des Drahtes sowie gegen das Aspektverhältnis (d)

Abbildung 4.21 zeigt die in den Defekten gespeicherte Anzahl von Atomlagen normiert auf die jeweilige Drahtlänge gegen den äquivalenten Durchmesser (a), die kurze (b) und lange (c) Facette sowie gegen das Aspektverhältnis (d) des Drahtquerschnitts. Klare Zusammenhänge sind mit keiner der Größen auszumachen. Allerdings scheint eine große Speicherung lediglich bei kleinen Drähten und Drähten mit einem homogenen Querschnitt, also kleinem Aspektverhältnis, zu erfolgen. Dies ist möglicherweise als Hinweis darauf zu interpretieren, dass die Querschnittsform Einfluss auf das Verformungsverhalten haben kann.

\section{Weitere Defekte}

Zusätzlich zu den planaren Defekten ließ sich in 6 Drähten eine Abgleitung entlang einer kristallographischen Ebene erkennen. In vier Fällen handelt es sich dabei um 


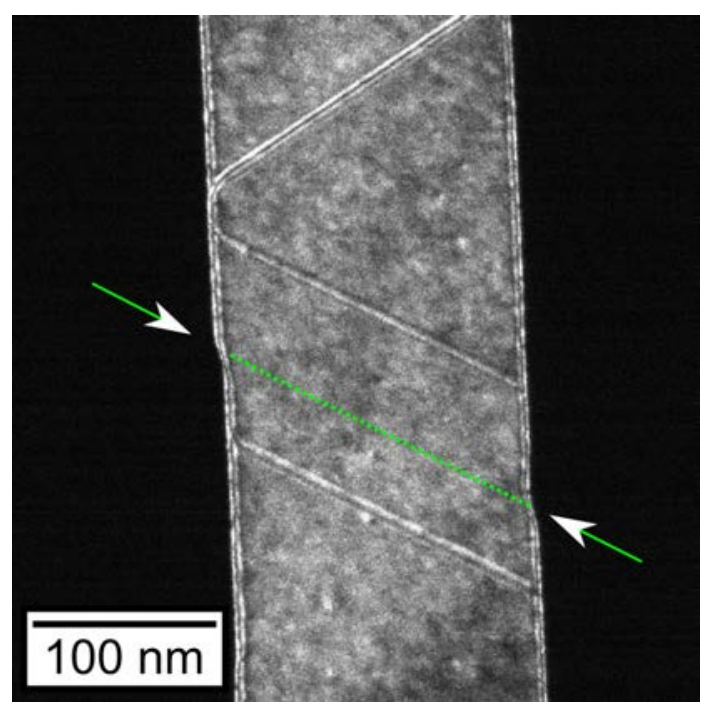

(a)

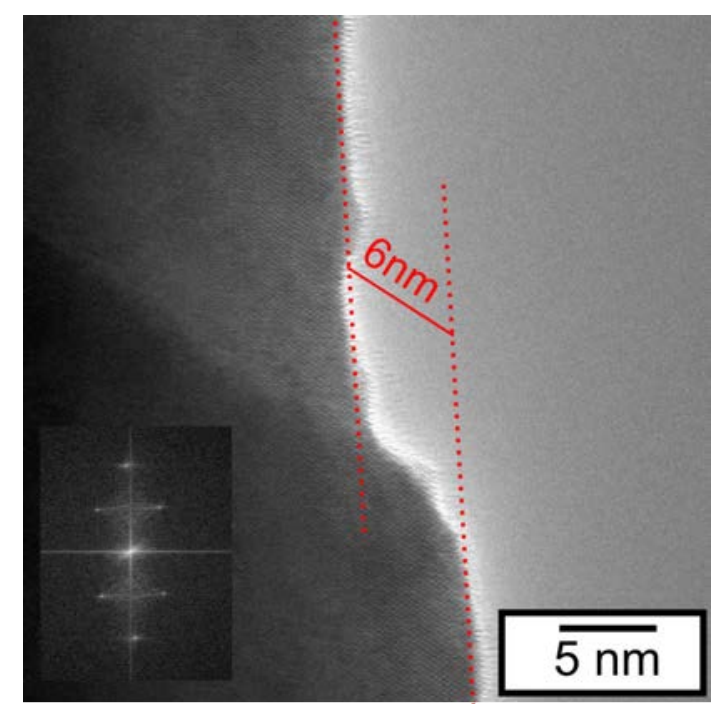

(b)

Abbildung 4.22.: Abgleitung: a) Abgleitung entlang einer kristallographischen Ebene in der (111)-Zonenachse; b) Hochauflösungsaufnahme an der Stufe bei der Oberfläche der Abgleitung in der (110)-Zonenachse; das Inset zeigt die Fouriertransformation

Drähte mit einem hohen Aspektverhältnis im Drahtquerschnitt, was auf den Einfluss der Querschnittsform auf das Verformungsverhalten hindeutet. In Abbildung 4.22 (a) ist die Abgleitung durch die gestrichelte grüne Linie markiert. Auf beiden Seiten des Drahtes kann man eine deutliche Stufe in der Oberfläche erkennen. Im Gegensatz zu den planaren Defekten ist kein Kontrast entlang der Abgleitung feststellbar, was vermuten lässt, dass kein Defekt in dem Draht gespeichert ist. Von einer Abgleitung konnte die Stufe an der Oberfläche in Hochauflösung untersucht werden (siehe Abbildung 4.22 (b)). Die Fouriertransformation (Inset in Abbildung 4.22 (b)) bestätigt, dass der Bereich einkristallin ist. Der Winkel zwischen Abgleitung und Drahtachse konnte hier zu $53^{\circ}$ ausgemessen werden und stimmt damit sehr gut mit dem theoretischen Winkel zu einer $\{111\}$-Ebene mit dem höchsten Schmidfaktor überein.

Weiterhin tauchten bei 11 Drähten Kontraste auf, die auf Versetzungen hindeuten. Abbildung 4.23 zeigt ein Beispiel dieser Kontraste. Diese waren ausschließlich nahe von Zwillingen feststellbar, wobei es sich dabei in den meisten Fällen um den Zwilling an der Bruchkante handelte. 


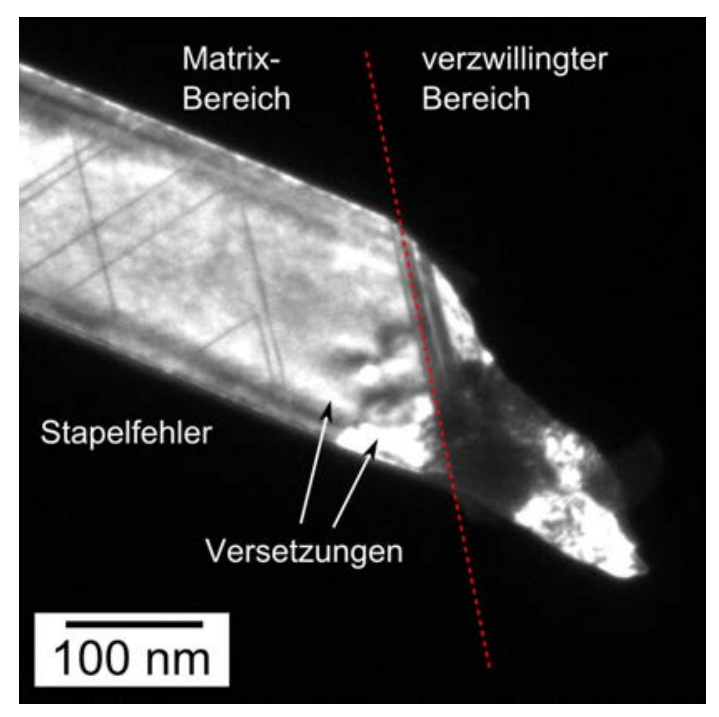

Abbildung 4.23.: Versetzungen nahe dem Zwilling an der Bruchkante

\subsubsection{Abschätzung der Dehnung}

Um die Anzahl von planaren Defekten mit dem zeitlichen Verlauf der Dehnung korrelieren zu können, wurden auf einem Draht nach dem Transfer mit elektronenstrahldeponiertem Platin insgesamt vier Markerpunkte aufgebracht. Während des Versuchs erfolgten zunächst in regelmäßigen Abständen Hellfeldaufnahmen von der gesamten Drahtlänge. Abbildung 4.24 stellt drei dieser Hellfeldaufnahmen dar: (a) zeigt den Draht, nachdem so gut wie jegliche Ausbeulung aus dem Draht verschwunden ist, also ungefähr am Anfang der elastischen Verformung; (b) zeigt den Draht direkt nach dem Erscheinen der ersten Defekte und (c) zeigt den Draht nach dem Reißen. Die Rissposition liegt nicht exakt mittig im Draht, jedoch zwischen den beiden äußeren Platinmarkern. Außerdem erkennt man, dass offensichtlich eine Fehlorientierung der Drahtachse zur Zugachse von $2^{\circ}$ vorlag. Weiterhin wird deutlich, dass der Draht nach dem Reißen eine Biegung aufweist.

Zur Abschätzung der elastischen Dehnung wurde in den ersten beiden Bildern jeweils der Abstand zwischen den äußeren Markern (in Abbildung 4.24 jeweils mit A bezeichnet) und den inneren Markern (mit Z markiert) bestimmt. Als ungefähres Maß für die elastische Dehnung ergibt sich $\epsilon_{\text {elastisch }_{A}}=(A 2-A 1) / A 1=1,7 \%$ bzw. $\epsilon_{\text {elastisch }_{Z}}=(Z 2-Z 1) / Z 1=1,6 \%$.

Nach dem Erscheinen der ersten Defekte wurde bis zum Reißen des Drahtes der Bereich zwischen den beiden inneren Markern verfolgt. So lässt lässt sich mit Hilfe von Digital Image Correlation sowohl die Verlängerung als auch die Dehnung von Bild 


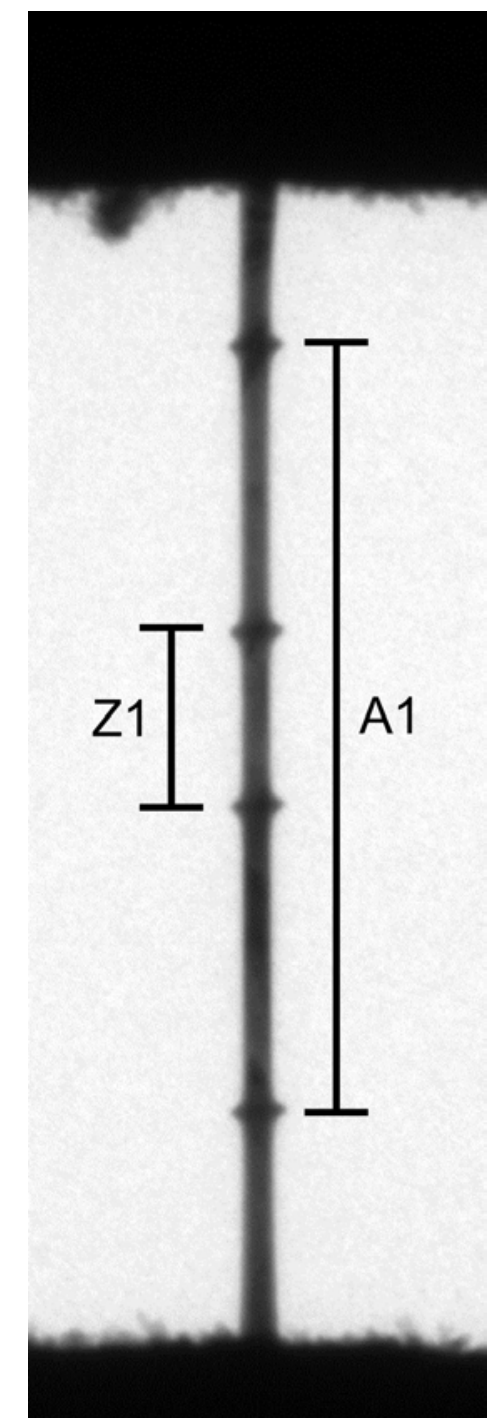

(a)

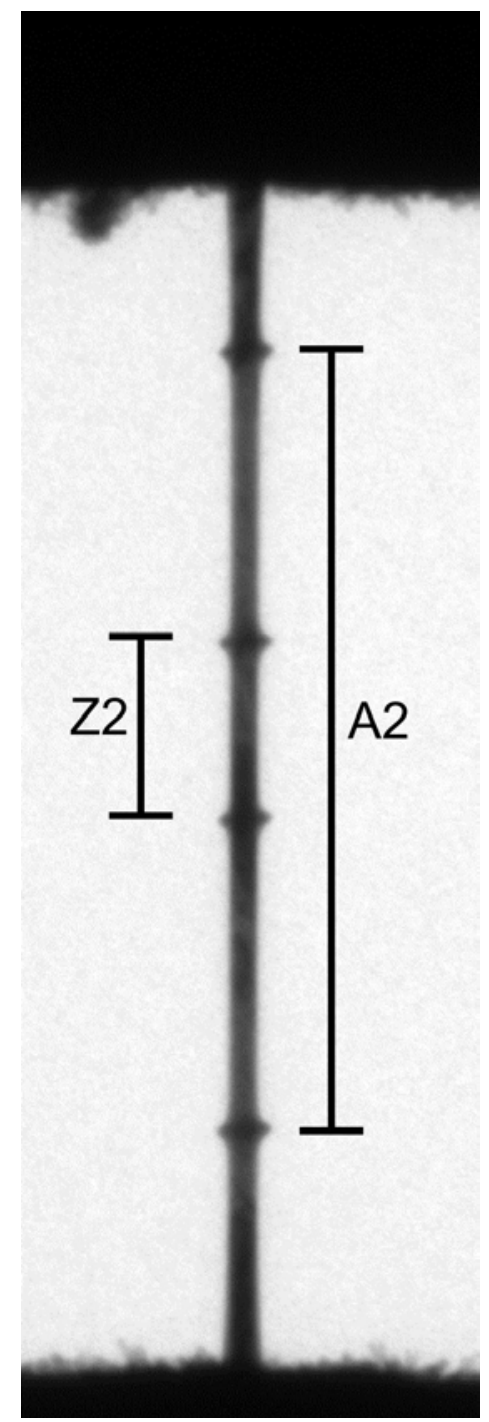

(b)

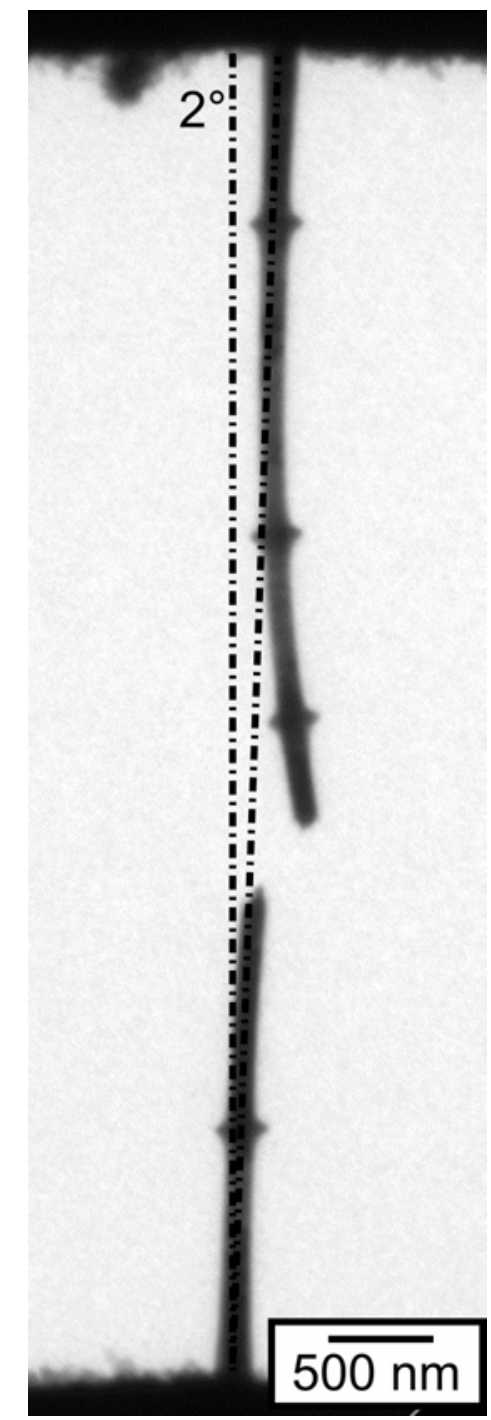

(c)

Abbildung 4.24.: Übersichts-Hellfeldaufnahmen von einem Draht mit Platin-Markern im Verlauf der Dehnung: a) zu Beginn der elastischen Verformung, b) nach dem Erscheinen der ersten Defekte, c) nach dem Reißen 
zu Bild zwischen den beiden inneren Markerpositionen verfolgen. Abbildung 4.25 (a) zeigt die Verlängerung bzw. eine Delta-Dehnung gegen die Zeit (Delta-Dehnung, da das Erscheinen der ersten Defekte als Nullpunkt gewählt wurde). (b) bis (d) zeigen jeweils eine TEM-Dunkelfeldaufnahme aus der Videoaufnahme. (e) zeigt den gleichen Bildausschnitt zusammengesetzt aus zwei Einzelbildern nach dem Reißen des Drahtes sowie ein zugehöriges Beugungsbild im Inset. Der jeweilige Zeitpunkt der TEM-Bilder ist in dem Plot in (a) markiert.

Zu Beginn von Video 1 befinden sich gerade 6 planare Defekte zwischen den beiden Pt-Markern, die einen Abstand von 903nm aufweisen (siehe Abbildung 4.25 (b)). Abbildung 4.25 (c) zeigt den Draht nach 16nm Verlängerung. Bis zu diesem Zeitpunkt entstehen nach und nach neue planare Defekte, bis in Abbildung 4.25 (c) 62 planare Defekte zwischen den beiden Pt-Markern gezählt werden können. Im weiteren Verlauf der Verformung kommt es ähnlich wie in Abbildung 4.12 immer wieder zum kollektiven Verschwinden von Gruppen planarer Defekte und anschließend wieder nach und nach zum Erscheinen neuer planarer Defekte. Eine Quantifizierung, wie viele planare Defekte verschwinden und neu entstehen, lassen die Abbildebedingungen allerdings nicht $\mathrm{zu}$.

Dieses Verhalten setzt sich fort bis zum Versagen des Drahtes. Abbildung 4.25 (d) zeigt den Draht in Video 4 kurz vor dem Reißen. Der Abstand der beiden Marker beträgt 1014nm, was einer Verlängerung von 109nm bzw. einer Delta Dehnung von $12 \%$ entspricht. Es lassen sich weiterhin einige planare Defekte zwischen den Markern ausmachen. Die Ausbildung eines ausgeprägten Zwillings ist jedoch auszuschließen, da die Oberfläche zwar scheinbar rauer geworden ist, eine deutliche Formänderung, wie sie typischerweise bei Zwillingen von mehr als $5 \mathrm{~nm}$ sichtbar ist (man vergleiche dazu Abbildung 4.9), ließ sich entlang des Drahtes nicht erkennen. Dies fand eine zusätzliche Bestätigung durch das Beugungsbild nach dem Versuch, welches keine Anzeichen für eine Verzwilligung liefert.

Abbildung 4.25 (e) zeigt den Draht schließlich nach dem Reißen. Der Abstand zwischen den beiden Markern beträgt 976nm, was einer Delta Dehnung von 7,8\% entspricht. Damit ist die Dehnung im Vergleich zum Zustand vor dem Reißen um mehr als $4 \%$ zurückgegangen, was deutlich über der abgeschätzten elastischen Dehnung von circa 1,7\% liegt. Die Diskrepanz könnte zum einen durch nichtlineare Elastizität erklärt werden, wie sie auch tatsächlich in den Spannungs-Dehnungs-Kurven festzustellen ist 


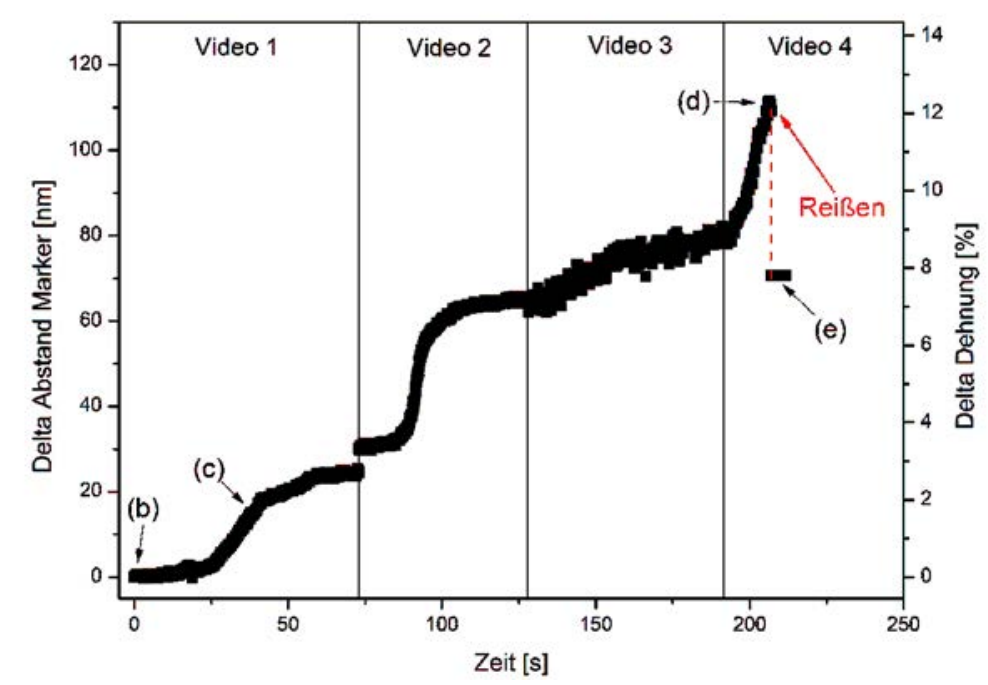

(a)

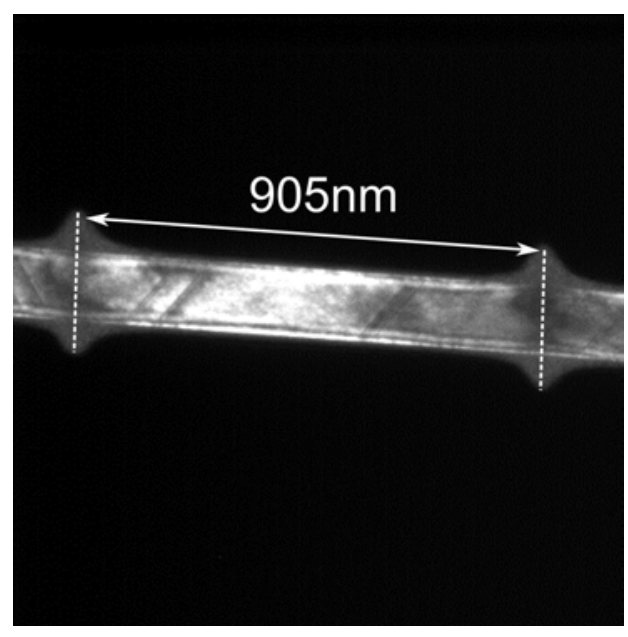

(b)

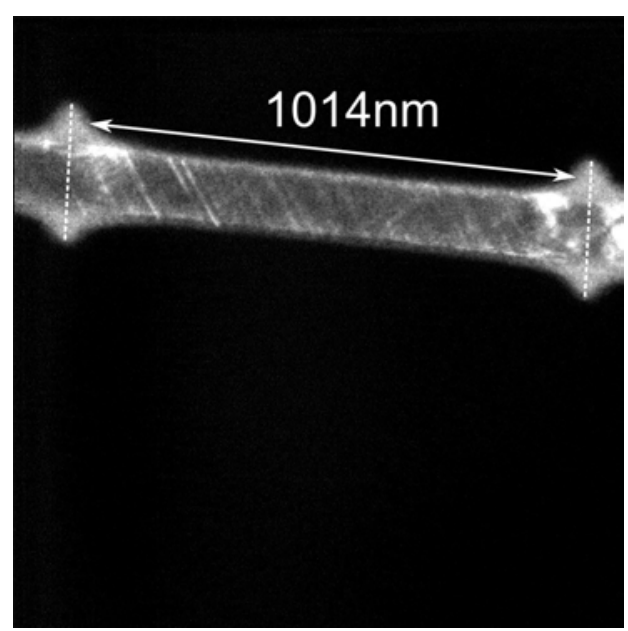

(d)

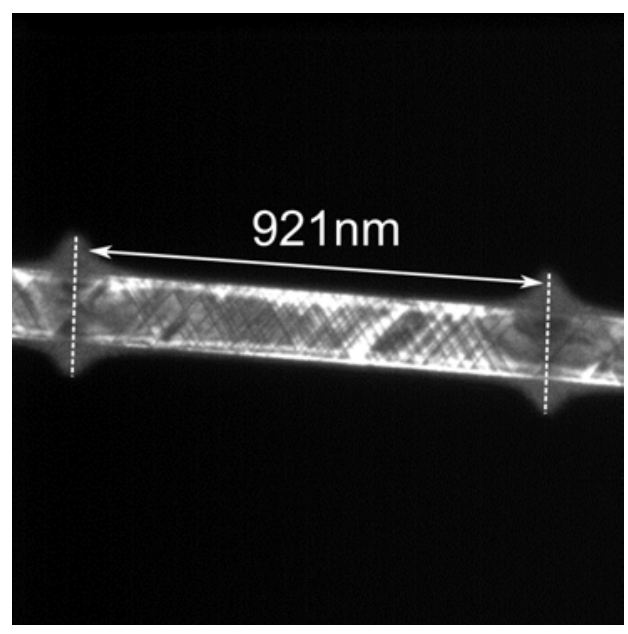

(c)

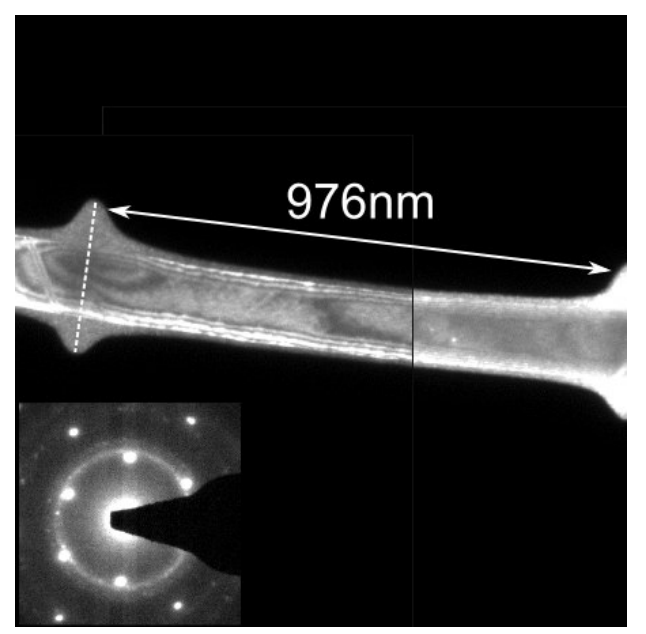

(e)

Abbildung 4.25.: Dehnung während eines TEM Versuchs: (a) Dehnung gegen die Zeit zwischen den beiden inneren Pt-Markern, (b) TEM-Dunkelfeldaufnahme zu Beginn von Video 1, (c) TEM-Dunkelfeldaufnahme in der Mitte von Video 1, (d) TEM-Dunkelfeldaufnahme am Ende von Video 4, (e) TEMDunkelfeldaufnahme nach dem Reißen des Drahtes 
(siehe Abschnitt 4.4.3). Zum anderen könnte es sich um eine Art reversibler Plastizität handeln. Diese Möglichkeit erfährt dadurch Bestärkung, dass ebenso wie in Abbildung 4.20 erkennbar das Verschwinden von planaren Defekten im Zuge des Reißens zu beobachten ist. 


\section{Zusammenfasssung: Verformung von einkristallinen Proben}

- In nahezu allen einkristallinen Proben (42/44) kommt es zu Speicherung von planaren Defekten auf zwei Sets von (111)-Ebenen aufgrund der Verformung. Bei diesen planaren Defekten handelt es sich entweder um Stapelfehler (1 oder 2 Ebenen) oder um Zwillinge ( $\geq 3$ Ebenen).

- In den in-situ TEM Tests lässt sich erkennen, dass die ersten planaren Defekte scheinbar bevorzugt an vor dem Versuch bereits vorhandenen Defekten entstehen, so dass die Defekt-Nukleation an diesen Stellen scheinbar begünstigt ist im Vergleich zu ungestörten Stellen. Während der gesamten weiteren Verformung ist das Erscheinen von weiteren planaren Defekten entlang des gesamten Drahtes festzustellen, so dass es scheinbar durchgehend zur Nukleation von identischen Defekten entlang des Drahtes kommt, räumlich unabhängig von bereits vorhandenen planaren Defekten.

- Neben dem Erscheinen von neuen planaren Defekten ist ebenfalls eine Verbreiterung von bereits vorhandenen planaren Defekten zu beobachten (9/10). Zwillinge mit einer Breite von $>5 \mathrm{~nm}$ waren jedoch nur in 2 Proben zweifelsfrei auszumachen. Nach dem Reißen lässt sich jedoch in dem Großteil der Drähte (32/36) ein Zwilling mit einer Breite zwischen 20nm und 200nm an der Bruchkante wahrnehmen. Weitere große Zwillinge $(>5 \mathrm{~nm})$ entlang der Drähte sind eher in der Minderheit (10/42). Damit führt bei einem Großteil der Drähte ein Zwilling ab einer bestimmten Größe zum Versagen des Drahtes oder umgekehrt.

- In nahezu allen Proben (10/11) war während der Verformung auch das Verschwinden von vorher entstandenen planaren Defekten zu registrieren. Dabei konnten planare Defekte sowohl einzeln als auch kollektiv verschwinden. Letzteres war nur in Bereichen mit einer hohen Dichte von planaren Defekten auf beiden $\{111\}$ Ebenen der Fall.

- Das Versagen der Drähte erfolgt einerseits abrupt, wobei sich innerhalb eines Kameraframes ein großer Zwilling bilden bzw. ein vorhandener stark vergrößern kann. Andererseits kam es zu einer kontinuierlichen Verjüngung über einen längeren Zeitraum in einem bestimmten Bereich des Drahtes. Hier erfolgte das Versagen schließlich unter Ausbildung eines dünnen Filaments in einer Art fließender 
Verformung.

- In sechs Proben war zu erkennen, dass planare Defekte im Zuge des Reißens wieder verschwinden. Dagegen war bei den beiden Drähten, bei denen eine Entlastung durchgeführt wurde, kein Hinweis auf ein Verschwinden nachzuweisen.

- Weitere Defektarten bilden im Vergleich zu planaren Defekten die Ausnahme: In 6 Proben konnte eine Abgleitung entlang einer $\{111\}$-Ebene gefunden werden, was auf das Gleiten von vollen Versetzungen hindeutet. Zudem ließen sich bei 11 Drähten Versetzungen beobachten. Diese lagen ausschließlich in der Nähe von Zwillingen, hauptsächlich in der Nähe des Zwillings an der Bruchkante.

- Vor dem Versuch erzeugte Versetzungen scheinen nach und nach aus dem Draht herauszulaufen und nicht zu einem Wechsel im Verformungsmechanismus zu führen. Anschließend kann man das Entstehen planarer Defekte entlang des gesamten Drahtes beobachten, wobei die ersten neuen planaren Defekte typischerweise in dem Bereich entstehen, in dem vorher die Versetzungen lagen. Eine Verjüngung als auch planare Defekte scheinen dagegen nicht als ausgezeichnete Orte für das Entstehen weiterer Defekte zu fungieren. 


\subsection{Verformungsverhalten von Drähten mit longitudinalen Zwillingsgrenzen}

In diesem Abschnitt wird das Verformungsverhalten von verzwillingten Drähten erörtert. Hierzu konnten die Ergebnisse von 15 in-situ Zugversuchen im TEM sowie die Ergebnisse von 7 post-mortem Analysen im REM getesteter Drähte herangezogen werden.

\subsubsection{Planare Defekte und Versetzungen}

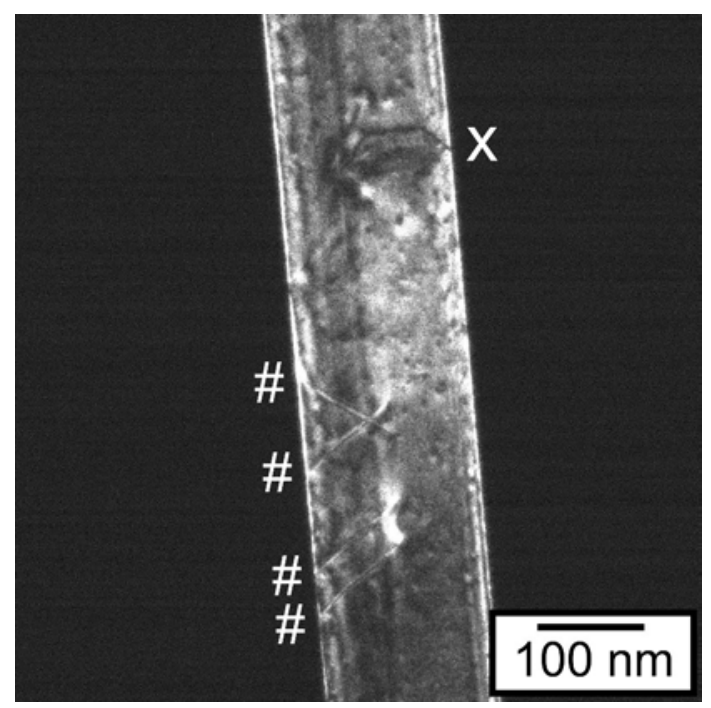

Abbildung 4.26.: Planare Defekte tauchen während der Verformung in beiden Zwillings-Orientierungen auf

Ebenso wie in einkristallinen Drähten ist während der Verformung in allen verzwillingten Drähten (15/15) ein sukzessives Auftauchen von planaren Defekten zu beobachten. Wie in Abbildung 4.26 ersichtlich, entstehen die planaren Defekte in beiden Zwillingsorientierungen (markiert durch ' $\mathrm{X}$ ' und '\#'). Ebenso wie bei den einkristallinen Drähten entstehen neue planare Defekte während der gesamten plastischen Verformung. Dagegen war ein Wachstum bzw. Verbreiterung der planaren Defekte während der Verformung nicht feststellbar, sondern nur im Zuge des Versagens (siehe Kapitel 4.3.4). Das Verschwinden von planaren Defekten ließ sich wie in einkristallinen Drähten beobachten, sowohl vereinzelt als auch mehrere planare Defekte kollektiv innerhalb eines Kameraframes. Wie bei den einkristallinen Drähten scheint auch hier eine Wech- 
selwirkung von planaren Defekten der beiden konjugierten Gleitebenen aufzutreten, wie in Abbildung 4.27 ersichtlich.

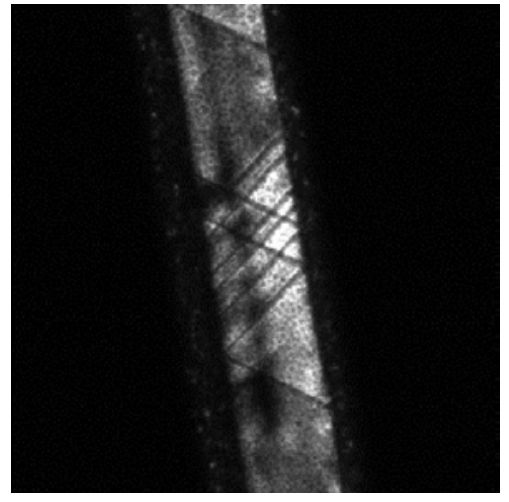

(a)

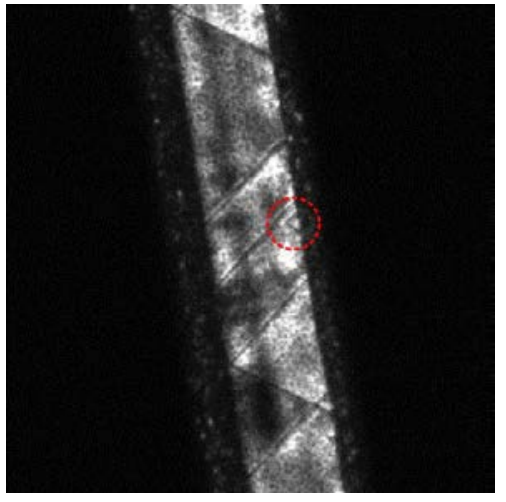

(b)

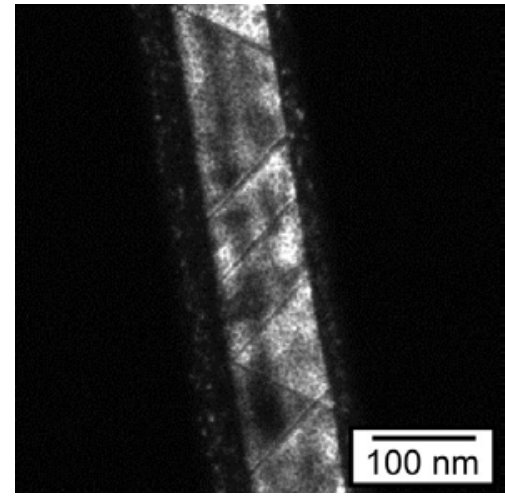

(c)

Abbildung 4.27.: Sequenz des Verschwindens von planaren Defekten in einem verzwillingten Draht über den Zwischenschritt einer Kombination von planaren Defekten der unterschiedlichen Gleitsysteme

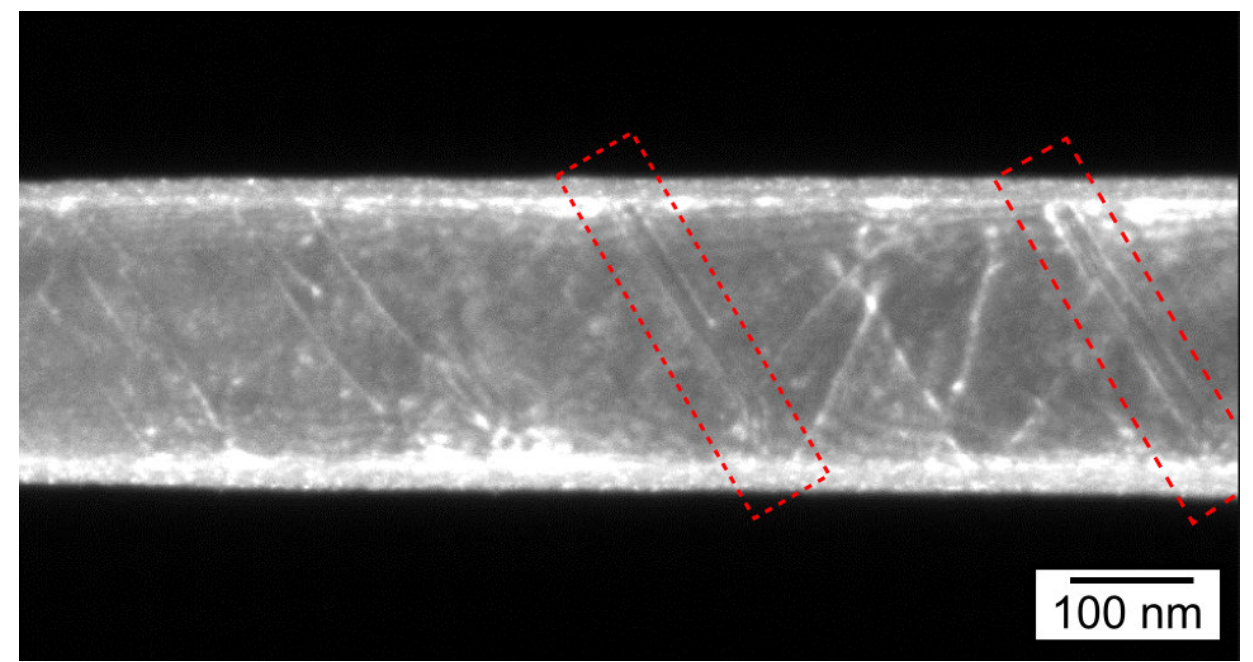

Abbildung 4.28.: Versetzungen und planare Defekte (markiert durch rot gestrichelte Boxen) während der Verformung in einem verzwillingten Drähten

Neben den planaren Defekten entstanden in 11 Proben während der Verformung auch Versetzungen in den Drähten. So kann man in Abbildung 4.28 sowohl zwei planare Defekte (markiert durch rot gestrichelte Boxen), die durch ihren oszillierenden Kontrast gut zu identifizieren sind, als auch eine ganze Reihe von feinen weißen Linien, bei denen es sich um Versetzungen handelt, erkennen.

Typischerweise scheinen planare Defekte zuerst zu entstehen, gefolgt von Verset- 


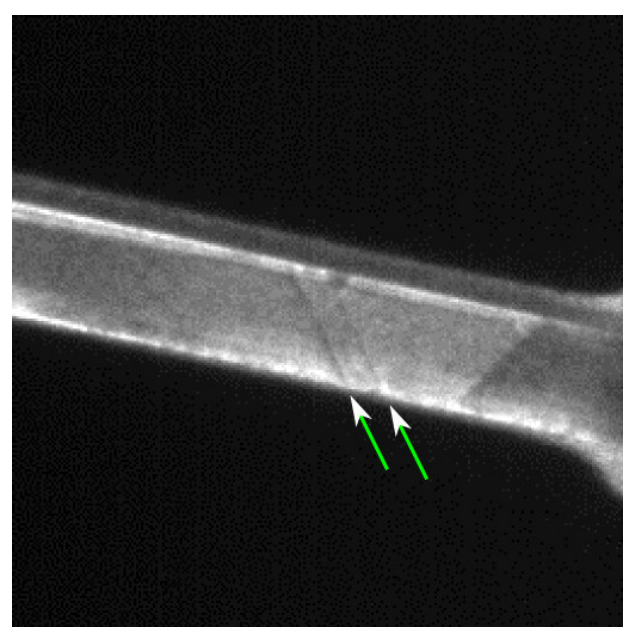

(a)

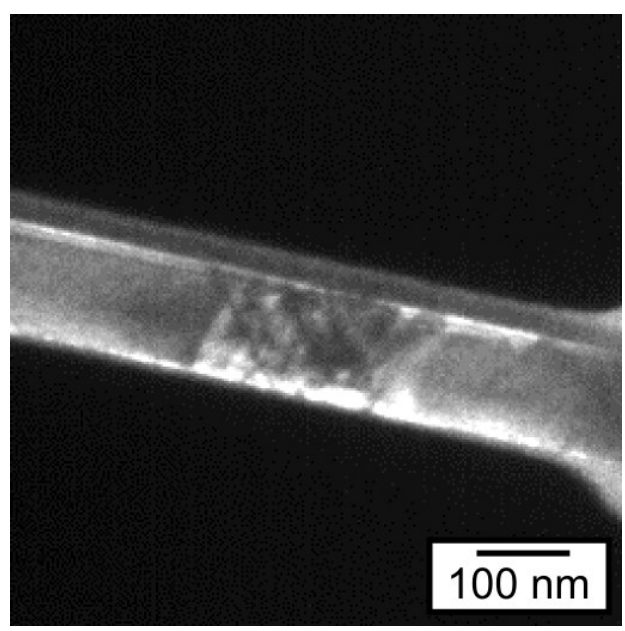

(b)

Abbildung 4.29.: Entstehen von Versetzungen an planaren Defekten: (a) zeigt zwei planare Defekte markiert durch Pfeile; (b) zeigt die Stelle 100ms später mit vielen neuen Kontrasten, die auf die Präsenz von vollen Versetzungen schließen lassen.

zungen. In einem Fall konnte beobachtet werden, wie innerhalb eines Kameraframes an zwei planaren Defekten viele neue Kontraste entstehen, die auf die Präsenz von Versetzungen schließen lassen, so dass vermutlich planare Defekte als Ausgangspunkte für Versetzungen dienen oder beim Verschwinden Versetzungen zurücklassen.

\subsubsection{Einfluss anfänglicher Defekte}

Wie in Abschnitt 4.1 beschrieben, ließen sich auch in den verzwillingten Drähten punktartige Kontraste nachweisen. Wie bei den einkristallinen Drähten scheinen auch hier planaren Defekte zuerst an diesen Stellen zu entstehen. So zeigt Abbildung 4.30 in (a) den Draht mit einem punktförmigen Defekt und in (b) den Draht nach dem Erscheinen des planaren Defektes. Somit scheint die Nukleation von Defekten ebenfalls in den verzwillingten Drähten durch die Anwesenheit von vorherigen Defekten begünstigt zu sein.

Neben punktartigen Kontrasten waren in den verzwillingten Proben auch einige Versetzungen vorhanden. So sind in Abbildung 4.31 zwei Versetzungen 'D1' und 'D2' zu sehen. Während der Verformung ist zunächst das gleichzeitige Verschwinden von der Versetzung 'D1' und das Erscheinen des planaren Defekts 'SF1' zu erkennen, während die Versetzung 'D2' unverändert bleibt. Mit weiterer Verformung verschwindet 'SF1' 


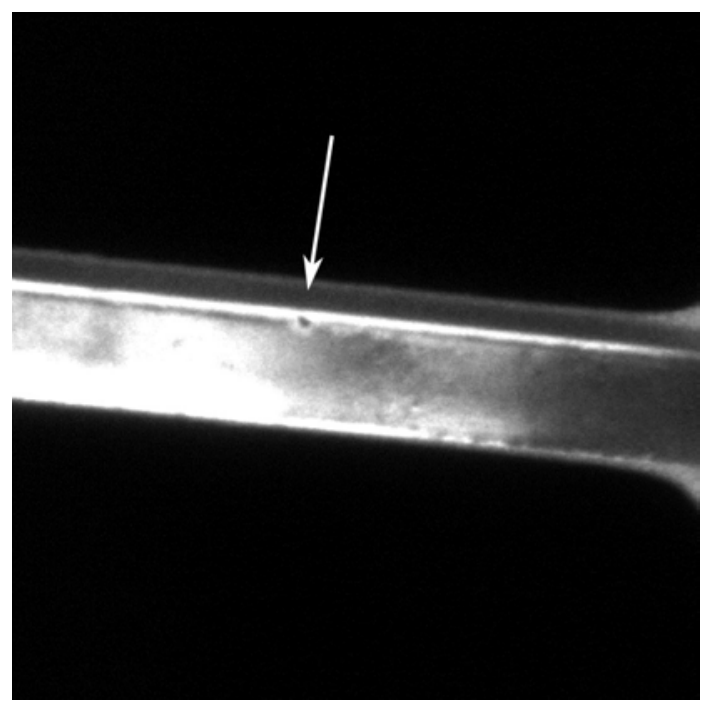

(a)

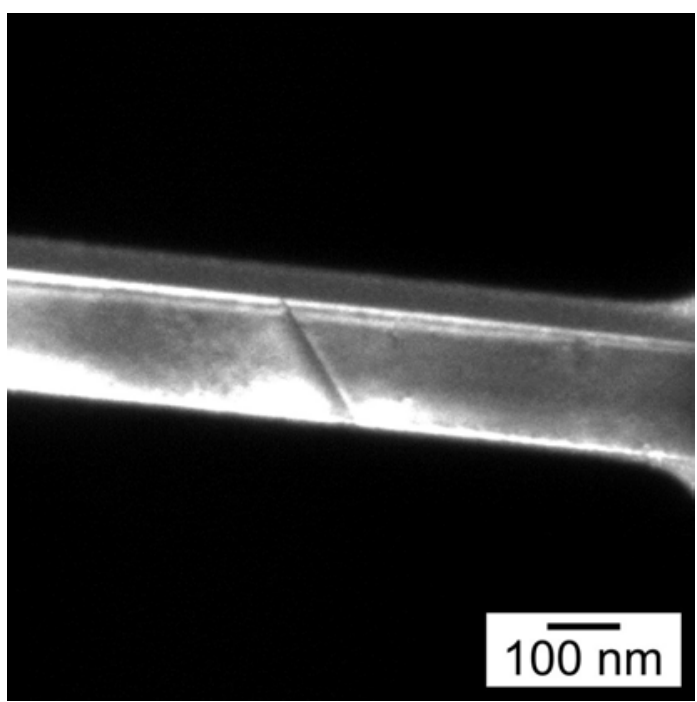

(b)

Abbildung 4.30.: Auftauchen eines planaren Defektes an einem punktförmigen Kontrast

wieder, wobei keine Kontraste auszumachen sind, die auf den Verbleib eines Defektes hindeuten. Anschließend entsteht am Endpunkt der Versetzung 'D2' ein weiterer planarer Defekt 'SF2', ohne dass die Versetzung 'D2' verschwindet. So scheinen Versetzungen ebenfalls ausgezeichnete Nukleationspunkte für das Auftauchen neuer Defekte zu sein. Was dabei mit den Versetzungen geschieht, kann dabei dem Anschein nach von Fall zu Fall variieren. In einem Fall hat die Versetzung den Draht offenbar verlassen, während in einem anderen Fall die Versetzung weiterhin im Draht gespeichert ist. In anderen Drähten waren Fälle zu beobachten, in denen Versetzungen während der Verformung komplett immobil geblieben sind.

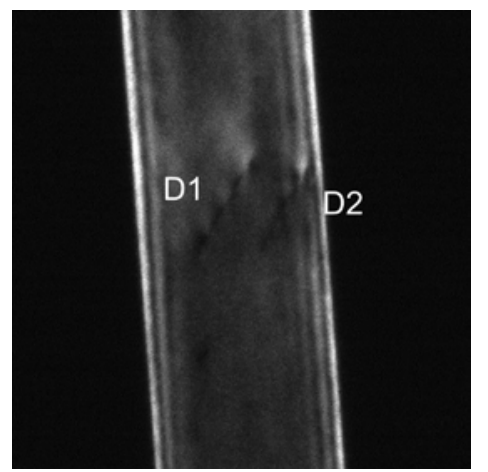

(a)

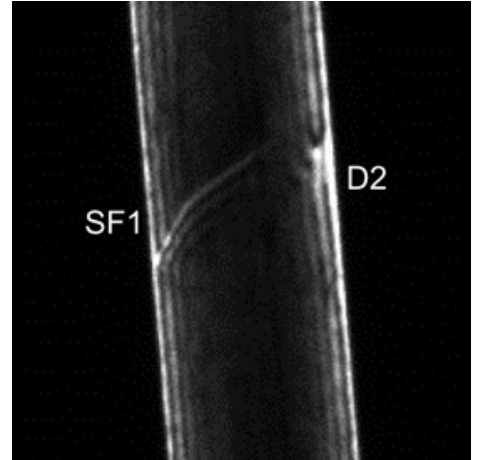

(b)

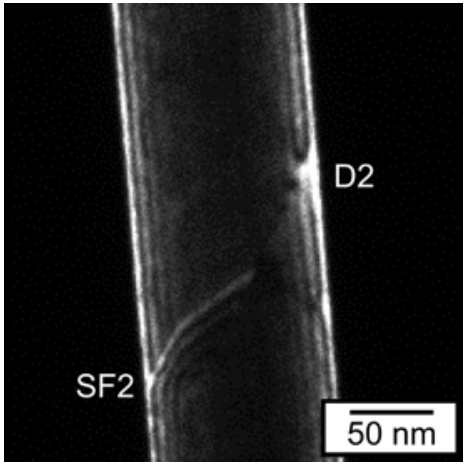

(c)

Abbildung 4.31.: Einleiten von neuen Defekten 
Um auch hier den Einfluss von Defekten auf das Verformungsverhalten weiter zu untersuchen, wurden in drei Proben vor dem Zugversuch Defekte, wie in Abschnitt 3.4 beschrieben, eingebracht. Dabei entsteht am Ort der maximalen Beanspruchung meist ein Netzwerk aus vielen Versetzungen, wie die vielen Kontraste in der Mitte von Abbildung 4.32 verdeutlichen. Dabei scheinen gewöhnlich mehr Versetzungen zu entstehen als im Fall einkristalliner Drähte, so dass es anscheinend einfacher ist Versetzungen in verzwillingte Drähte einzubringen oder zu speichern. Neben dem Netzwerk in der Mitte des Drahtes konnten auch vereinzelte Versetzungen entlang des Drahtes nachgewiesen werden. Da auch in den Drähten ohne Beanspruchung teilweise Versetzungen vorkommen, ist in diesen Fällen nicht klar, ob diese durch die Beanspruchung entstanden sind. Neben den Versetzungen ist auch immer eine kleine Verjüngung des Drahtes zu erkennen, die vom Kontakt mit dem 'Indenter' herrührt.

Im Zuge der Verformung kann man gewöhnlich zunächst Kontraständerungen im Bereich der Defekte beobachten. Diese Kontrastwechsel sind dabei eher ruckartig und deuten auf die Aktivität von Versetzungen hin. Im weiteren Verlauf der Verformung kann man entlang des Drahtes ebenfalls neue Defekte erkennen, bei denen es sich sowohl um planare Defekte als auch um Versetzungen handelt (siehe Abbildung 4.32 (b)). Ob sich auch planare Defekte in der Mitte des Drahtes bilden, lässt sich aufgrund vieler Überlagerungen von Kontrasten nicht feststellen. Die eingebrachten Versetzungen scheinen also auch im Fall von verzwillingten Drähten keine Änderung im Verformungsmechanismus zu verursachen. Weiterhin führt die Verjüngung des Drahtquerschnitts augenscheinlich nicht zu einer derartigen Spannungskonzentration, dass es ausschließlich zu einer Verformung im verjüngten Bereich kommt.

\subsubsection{Verhalten bei Entlastung}

Bei insgesamt 7 Drähten erfolgte nach vorangegangener Zugdehnung jeweils eine Entlastung. Dazu wurden die beiden Aufnahmepunkte des Zughalters wieder zusammengefahren. Der Verfahrweg und damit die Entlastung der Drähte war dabei jeweils so bemessen, dass es nicht zu einer Kompression bzw. Biegung des Drahtes kam, der Draht also weiterhin unter einer Zugspannung stand. In allen Fällen konnte beobachtet werden, dass im Zuge der Entlastung nach und nach planare Defekte, die sich während der Verformung gebildet hatten, wieder verschwanden. Das Verschwinden der planaren Defekte muss dabei nicht innerhalb eines Kameraframes geschehen, sondern kann sich 


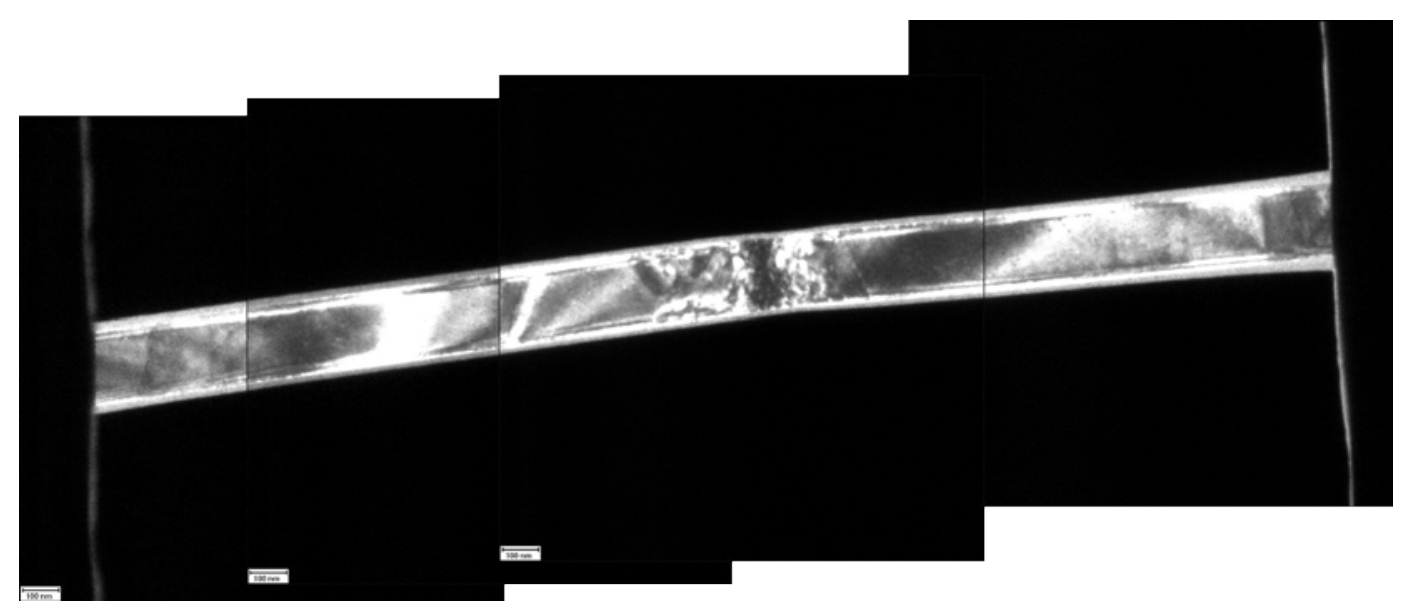

(a)

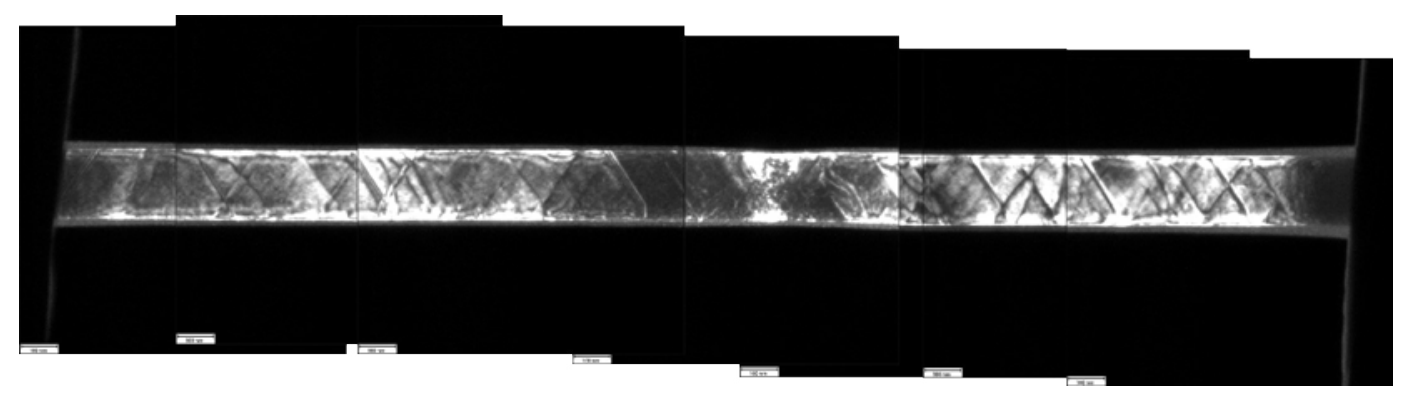

(b)

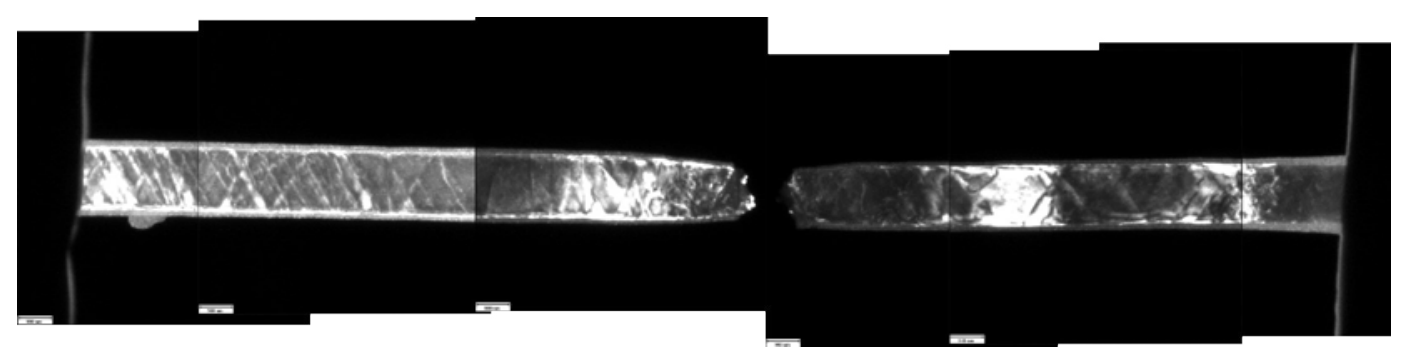

(c)

Abbildung 4.32.: Zugversuch an Draht mit Zwillingsgrenze und Vorverformung 
über mehrere Frames erstrecken (aber $<5$ Frames). Für Versetzungen ließ sich ein solches Verhalten nicht erkennen.

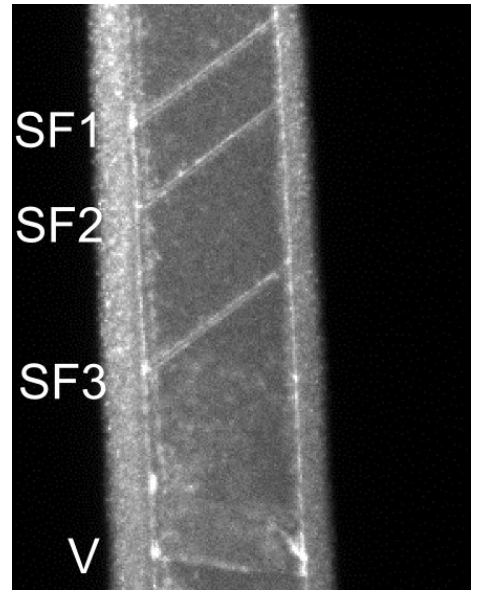

(a)

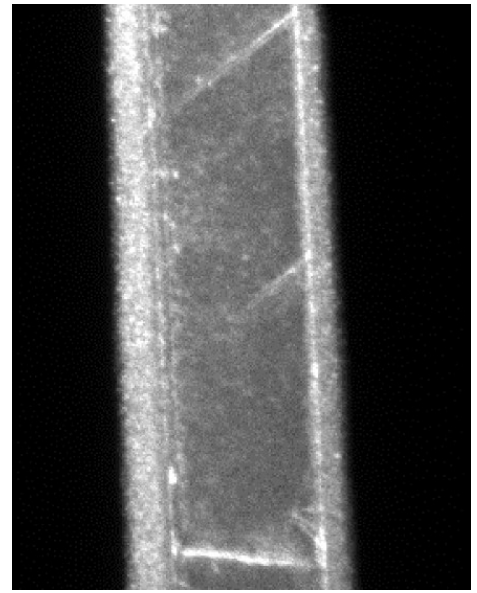

(b)

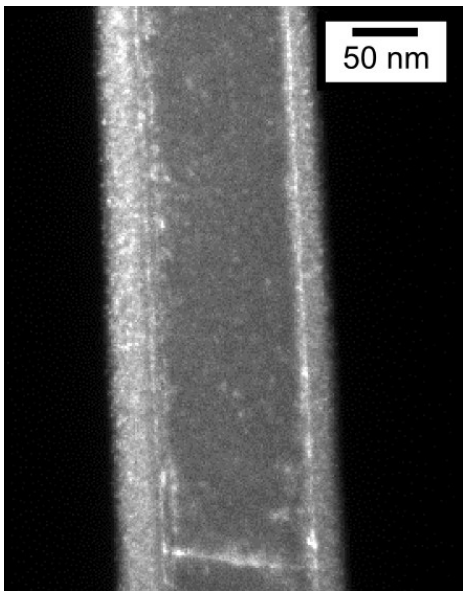

(c)

Abbildung 4.33.: Sequenz zur Entlastung bei einem Draht mit Zwillingsgrenze

Abbildung 4.33 zeigt die Sequenz einer solchen Entlastung, bei der insgesamt 3 planare Defekte verschwinden. In Abbildung (a) sind sowohl die drei planaren Defekte 'SF1', 'SF2' und 'SF3' sowie eine Versetzung 'V' zu sehen. In Abbildung (b) ist zunächst der planare Defekt 'SF2' sowie der planare Defekt 'SF3' zur Hälfte verschwunden. Im Abbildung (c) sind letztlich alle drei planare Defekte nicht mehr vorhanden. Die Versetzung 'V' bleibt während der Entlastung ortsfest.

\subsubsection{Versagen und Bruchmorphologie}

Nach dem Versagen scheint eine Speicherung von planaren Defekten nur in Ausnahmen zu erfolgen. Diese ließen sich nur in 4 Proben beobachten. Zudem wurden in drei dieser Proben maximal 2 planare Defekte entlang des Drahtes gefunden. Eine Ausnahme bildet ein Draht, in dem eine Speicherung von mehreren planaren Defekten entlang der Drahtlänge ähnlich wie im Fall von einkristallinen Drähten auftrat. Auffällig ist in diesem Fall, dass der Querschnitt dieses Drahtes im Gegensatz zu den meisten anderen verzwillingten Drähten sehr homogen mit einem Aspektverhältnis von 1,2 ist, so dass sich auch hier eventuell einen Hinweis auf den Einfluss der Querschnittsform der Drähte ergibt.

Im Gegensatz zu planaren Defekten bleiben Versetzungen im Draht gespeichert. Sie konnten in 21 Proben nach dem Versagen nachgewiesen werden entsprechend Ab- 
\begin{tabular}{l|l} 
Planare Defekte während Verformung & Planare Defekte nach dem Reißen \\
\hline
\end{tabular}

$15 / 15$

$4 / 22$

Tabelle 4.2.: Planare Defekte in Drähten mit Zwillingsgrenze

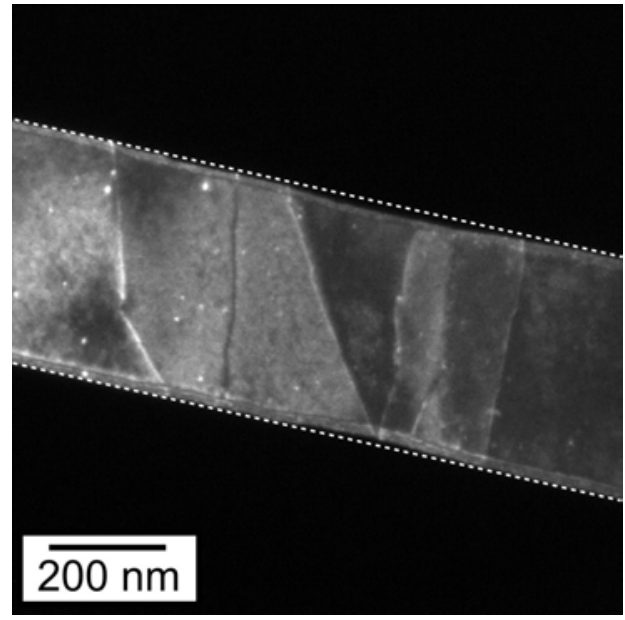

(a)

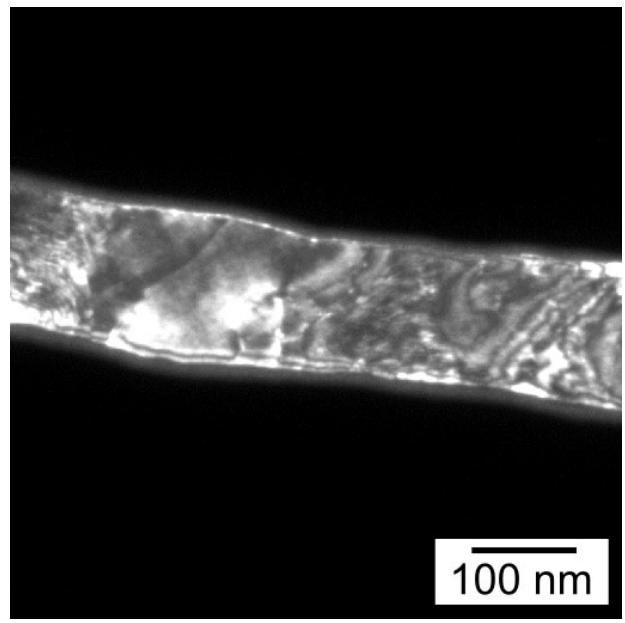

(b)

Abbildung 4.34.: Versetzungen nach dem Reißen

bildung 4.34. Neben der Speicherung von Versetzungen kommt es zu kleinen Dellen in der Oberfläche. Diese sind insbesondere an den Stellen zu finden, an denen die Versetzungen im Draht gespeichert sind, wie in Abbildung 4.34 (a) zu sehen ist. Im Fall einer starken Verformung kann es auf diese Weise zu einer Art Aufrauung der gesamten Drahtoberfläche kommen (siehe Abbildung 4.34 (b)).

\begin{tabular}{c|c|} 
Versetzungen während der Verformung & Versetzungen nach dem Reißen \\
\hline $11 / 12$ & $21 / 21$
\end{tabular}

Tabelle 4.3.: Versetzungen während der Verformung in Drähten mit Zwillingsgrenze

Versetzungen können einerseits sehr komplexe Formen und Strukturen annehmen und scheinbar willkürlich im Draht liegen (siehe Abbildung 4.34 (b) und 4.28). Andererseits konnten aber in all den Fällen Versetzungen beobachtet werden, bei denen das Linienelement nahezu gerade und senkrecht zur Drahtachse liegt, wie in Abbildung 4.34 (a) und Abbildung 4.35 zu sehen ist.

Um den Charakter dieser Versetzungen weiter zu untersuchen, wurde eine Versetzung in zwei verschiedenen Zonenachsen (Abbildung 4.35 obere Reihe (110)-Zoneneachse, untere Reihe (111)-Zonenachse) unter jeweils drei verschiede- 

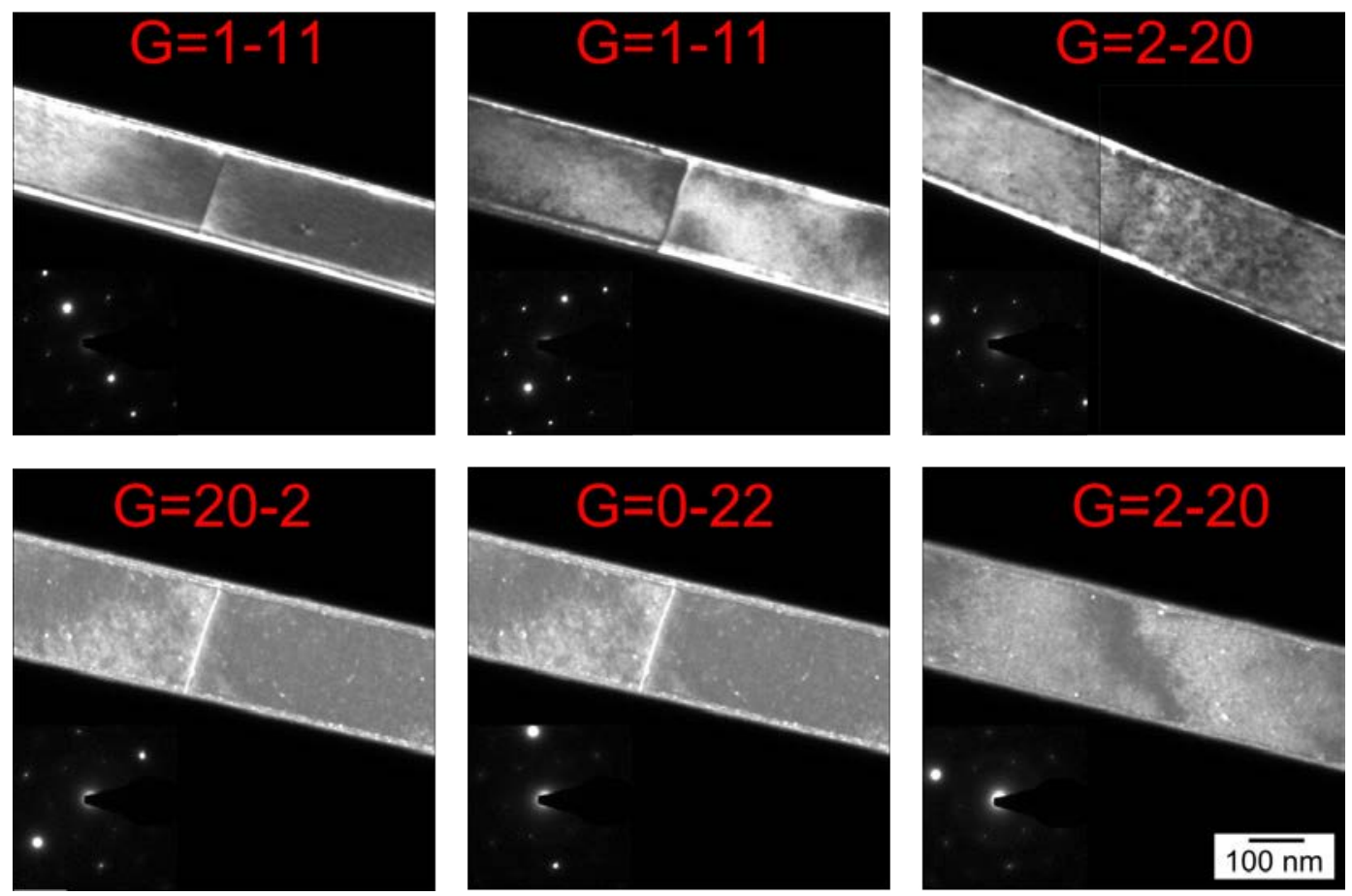

Abbildung 4.35.: Senkrecht zur Drahtachse liegende Versetzung: obere Reihe (110)Zonenachse, untere Reihe (111)-Zonenachse. g x b Auslöschungkriterium ergibt, dass der Burgersvektor der Versetzung ebenfalls senkrecht zur Drahtachse liegt. 
nen Anregungsvektoren für den Zweistrahlfall abgebildet. Mit Hilfe des (g x b)Sichtbarkeitskriteriums [57] konnte so bestimmt werden, dass der Burgersvektor senkrecht zur Drahtachse liegt und somit keine Scherspannung auf die Versetzung wirkt. In fcc-Metallen sind die mobilen Versetzungen entweder vom Typ einer vollen $\langle 110\rangle$ Versetzung oder $\langle 112\rangle$-Partialversetzung. Die beiden potentiellen $\langle 110\rangle$-Versetzungen mit einem Burgersvektor senkrecht zur Drahtachse existieren nur auf geneigten Ebenen zur Drahtachse und kommen somit aufgrund der Lage des Linienelementes nicht in Frage. Es bleibt somit die Möglichkeit, dass es sich um eine Partialversetzung handelt, die genau in der Ebene der Zwillingsgrenze liegt oder die Möglichkeit, dass es sich um eine immobile Versetzung handelt.

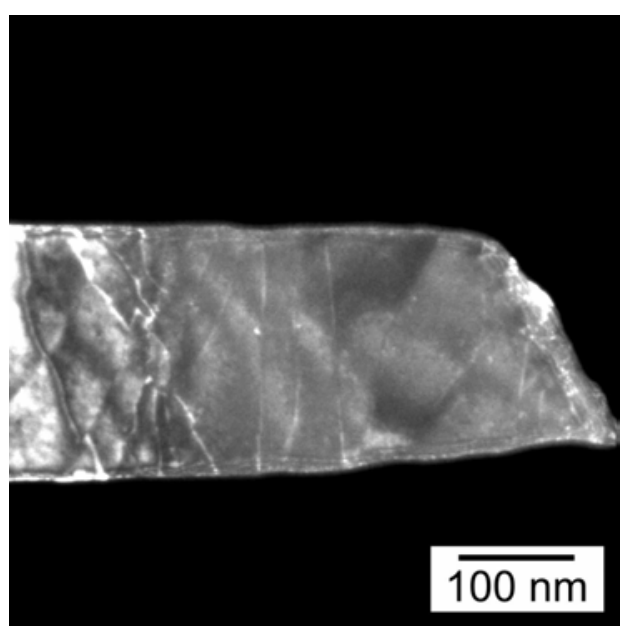

(a)

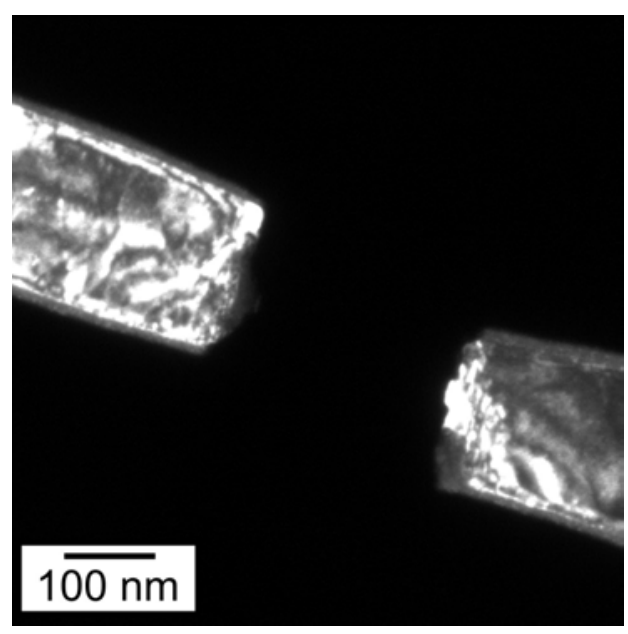

(b)

Abbildung 4.36.: Bruchmorphologie: (a) Scherbruch entlang einer kristallographischen Ebene, (b) Bruchkante senkrecht zur Drahtachse

Bei der Bruchmorphologie handelt es sich größtenteils um einen Scherbruch entlang einer kristallographischen Ebene, wie Abbildung 4.36 (a) zeigt. Bei den Drähten, in denen schon vorher Versetzungen eingebracht worden sind, kann es außerdem zu einem Bruch senkrecht zur Drahtachse kommen, wie Abbildung 4.36 (b) verdeutlicht.

In 6 Fällen konnte ein Zwilling an der Bruchkante ausgemacht werden. Weitere Zwillinge entlang der Drähte waren in keinem Fall nachweisbar. Der Zwilling an der Bruchkante scheint sich somit nur im Zuge des Versagens auszubilden, nachdem es im Draht an dieser Stelle bereits zu einer Verjüngung bzw. Abscherung gekommen ist. In einem Fall ließ sich diese Zwillingsbildung direkt beobachten. Abbildung 4.37 (a) zeigt diesen Draht kurz vor der größeren Abscherung. Der Draht ist an dieser Stelle durch 


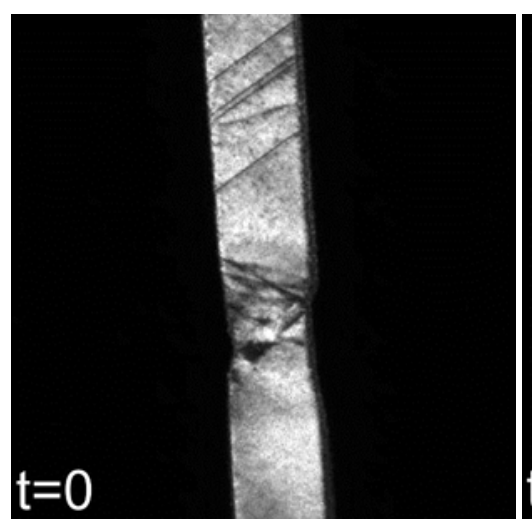

(a)

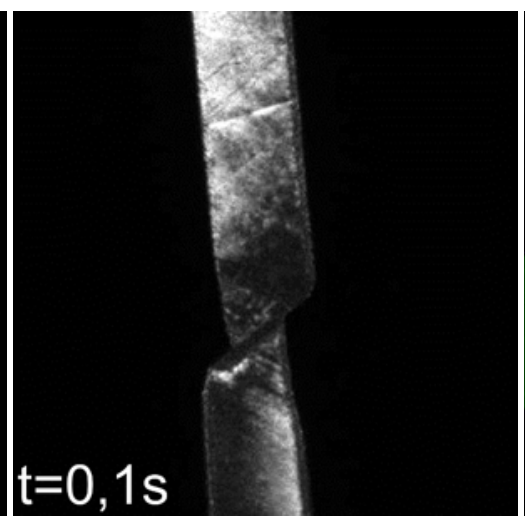

(b)

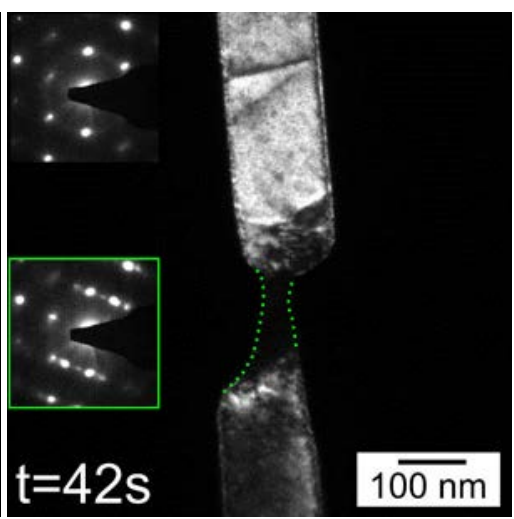

(c)

Abbildung 4.37.: Scherbruch mit Zwilling: (a) Zeigt den Draht vor dem Scherevent, (b) zeigt den Draht nach dem Scherevent mit einem kleinen Zwillingsbereich zwischen den abgescherten Segmenten, (c) zeigt den Draht kurz vor dem Versagen

die Verformung bereits leicht verjüngt und die Kontraste lassen auf Versetzungen in dem verjüngten Bereich schließen. Innerhalb eines Frames (100ms) schert der Draht in dem verjüngten Bereich entlang einer Ebene ab (siehe 4.37 (b)). Ein Ausmessen des Winkel ergibt $56^{\circ}$, was für eine $\{111\}$-Ebene spricht (theoretischer Winkel $54,7^{\circ}$ ). Ein schmales dunkles Band hat sich zwischen den beiden abgescherten Bereichen gebildet. Bei weiteren Verfahrschritten am Halter kommt es zu einer Art fließendem Wachstum des dunklen Bandes zwischen den beiden Segmenten (siehe 4.37 (c)). Beugungsbilder zeigen, dass es sich bei diesem dunklen Bereich um einen verzwillingten Bereich handelt (siehe Insets von 4.37 (c)). Finales Versagen erfolgt letztlich in der Mitte des dunklen Bereiches. Es ist zu erkennen, dass die vier planaren Defekte in Abbildung 4.37 (a) nach dem Scherevent zumindest noch teilweise sichtbar sind (b), in (c) jedoch verschwunden und auch nach dem Versagen nicht mehr zu beobachten sind. Im Gegensatz dazu verbleiben die beiden Versetzungen während des Scherevents und auch beim finalen Versagen fest an ihrem Ort. Hierbei ist zu beachten, dass das schräge Versetzungssegment, das an das senkrecht zur Drahtachse liegende Versetzungssegment anknüpft, bereits in (a) vorhanden und nur durch die Abbildebedigungen nicht sichtbar ist. 


\section{Zusammenfassung: Verformung von Proben mit Zwillingsgrenze}

- Während der Verformung erscheinen in beiden Zwillingsorientierungen planare Defekte (15/15). Die ersten planaren Defekte entstehen auch hier vorzugsweise an vorhandenen punktartigen Defekten.

- Während der Verformung lässt sich beobachten, dass planare Defekte wieder verschwinden (15/15). Nach dem Versagen konnten in den Drähten nur in Ausnahmefällen planare Defekte festgestellt werden und auch hier nur in sehr geringer Anzahl (4/22). Gleichzeitig war in Entlastungsversuchen ebenfalls eine Rückbildung von planaren Defekten zu erkennen (8/9), so dass im Fall der verzwillingten Drähte das Abnehmen der Spannung sowohl beim Reißen als auch beim Entlasten zu einem ähnlichen Verhalten führt.

- Verbreiterte planare Defekte $(>5 \mathrm{~nm})$ konnten während der Verformung nicht nachgewiesen werden. Nach dem Reißen war eher in Ausnahmen an der Bruchkante ein breiter planarer Defekt (6/20) auszumachen. Weitere breite planare Defekte entlang des Drahtes konnten in keinem Fall gefunden werden, was darauf hindeutet, dass der Mechanismus zur Verbreiterung erschwert ist und nur in Verbindung mit dem Versagen auftreten kann.

- Versetzungen ließen sich während der Verformung in nahezu allen Proben beobachten (11/12). Nach dem Reißen ergab sich eine Speicherung der Versetzungen in allen Proben (21/21). Diese Versetzungen können auf der einen Seite sehr komplexe Formen aufweisen und offenbar willkürlich im Draht vorliegen. Auf der anderen Seite können Versetzungen augenscheinlich in einer senkrechten Anordnung zur Drahtachse vorliegen. Der Burgersvektor dieser Versetzungen liegt senkrecht zur Drahtachse. 


\subsection{In-situ REM Versuche}

In diesem Abschnitt erfolgt die Darstellung der Ergebnisse der in-situ REM Versuche. Hierbei stehen zunächst die beiden Größen Dehnung und Spannung jeweils separat im Fokus. Anschließend folgt die Darstellung der Spannungs-Dehnungs-Kurven und der daraus gewonnenen Größen wie Elastizitätsmodul, Fließspannung und plastische Dehnung. Insgesamt wurde das Spannungs-Dehnungs-Verhalten von 39 Drähten (31 einkristalline, 8 verzwillingte Drähte) ausgewertet.

\subsubsection{Dehnung}

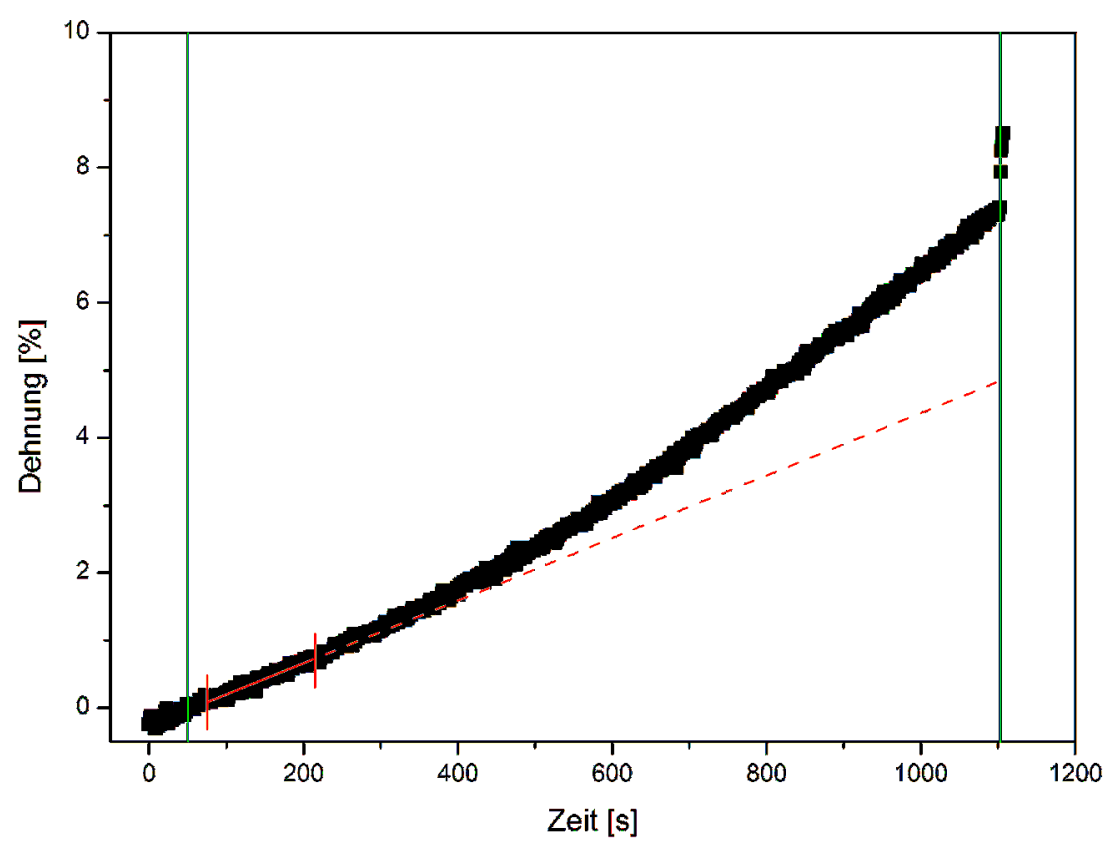

Abbildung 4.38.: Dehnung gegen Zeit exemplarisch für AuNw25: Die beiden grünen Linien markieren das Erreichen der Messlänge, nachdem der Draht gerade gezogen ist, sowie das Reißen des Drahtes. Die durchgezogene rote Linie zeigt den linearen Fit zur Bestimmung der Dehnrate mit der gestrichelten roten Linie als Verlängerung.

Die Dehnung wird mittels Digital Image Correlation aus den Einzelbildern, wie in Abschnitt 3.3.2 beschrieben, gewonnen. Mit Hilfe der bekannten Aufnahmerate der Bilder während des Versuchs ergibt sich damit die Dehnung als Funktion der Zeit. Üblicherweise lag die Aufnahmerate der Bilder bei ein bis zwei Bildern pro Sekunde, so dass auch die Dehnungswerte mit einer Rate von $1 \mathrm{~Hz}$ bis $2 \mathrm{~Hz}$ vorliegen. Abbildung 
4.38 zeigt exemplarisch die Dehnung gegenüber der Zeit von AuNw25. Als Nullpunkt der Dehnung wurde dabei der Zeitpunkt gewählt, an dem der Draht gerade gezogen und jegliche Biegung aus dem Draht verschwunden war. Dieser Punkt ist durch die erste grüne Linie gekennzeichnet und das zugehörige REM-Bild dient der Bestimmung

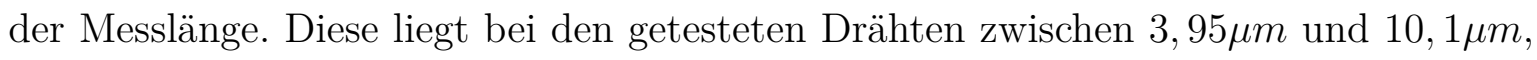
wobei der Großteil der Drähte eine Messlänge zwischen $8 \mu m$ und $10 \mu m$ aufweist. Das Reißen des Drahtes ist erneut durch eine grüne Linie gekennzeichnet.

Zu Beginn des Tests erkennt man eine kleine negative Dehnung. Dies liegt an der Markerposition auf den Pt-Pflastern bei der DIC: Der Draht weist zu diesem Zeitpunkt noch eine leichte Biegung auf, steht jedoch nicht unter Kompression. Nach dem Geradeziehen des Drahtes steigt die Dehnung zunächst linear an, wie es auch durch die lineare Aktuation des Positionieres zu erwarten ist. In diesem linearen Bereich, genauer zwischen einer Dehnung von 0,1\% und 0,75\%, wurde für alle Drähte die Dehnrate mit Hilfe einer linearen Regression ermittelt. Diese ist in Abbildung 4.38 durch eine rote Linie gekennzeichnet. In Fall von AuNw25 liegt die Dehnrate zum Beispiel bei $4,6 \cdot 10^{-5} / \mathrm{s}$. Im Zuge der Untersuchungen wurde die Geschwindigkeit des Aktuators in der Weise variiert, dass die Dehnraten der getesteten Drähte zwischen $1 \cdot 10^{-5} / \mathrm{s}$ und $4 \cdot 10^{-4} / s$ lagen und damit etwas mehr als eine Größenordnung umfassen.

Nach anfänglichem linearen Anstieg nimmt die Steigung ab einer Dehnung von circa $1 \%$ stetig zu und weicht damit immer weiter von dem linearen Verlauf, angedeutet durch die gestrichelte rote Linie, ab, ohne dass die Geschwindigkeit des Aktuators während des gesamten Verlaufs verändert wurde. Die Abweichung, sofern man ein Rutschen unter den Platin-Pflastern ausschließt, kann also nur durch den Kraftsensor hervorgerufen werden. Eine mögliche Erklärung könnte in dem Beginn der plastischen Verformung oder in einer nicht-linearen Elastizität des Drahtes zu finden sein.

Ab circa 4\% Dehnung steigt diese erneut nur noch linear an, allerdings mit einer höheren Dehnrate als zu Beginn des Tests. Das Reißen des Drahtes erfolgt schließlich bei einer Dehnung von circa 7,4\%. Diese maximale Dehnung variiert dabei stark zwischen den einzelnen Versuchen und reicht von 2,9\% bis zu 17,9\%. Nach dem Reißen des Drahtes springt der Kraftsensor wieder zurück in seine Ursprungsposition, so dass auch ein Sprung in der Dehnung zu sehen ist. Die Größe des Sprungs hängt dabei von der Kraft beim Reißen und damit vom Durchmesser des Drahtes ab, wobei dünne Drähte der Federsteifigkeit des Sensors entsprechend kleinere Sprünge aufweisen. 


\subsubsection{Spannung}

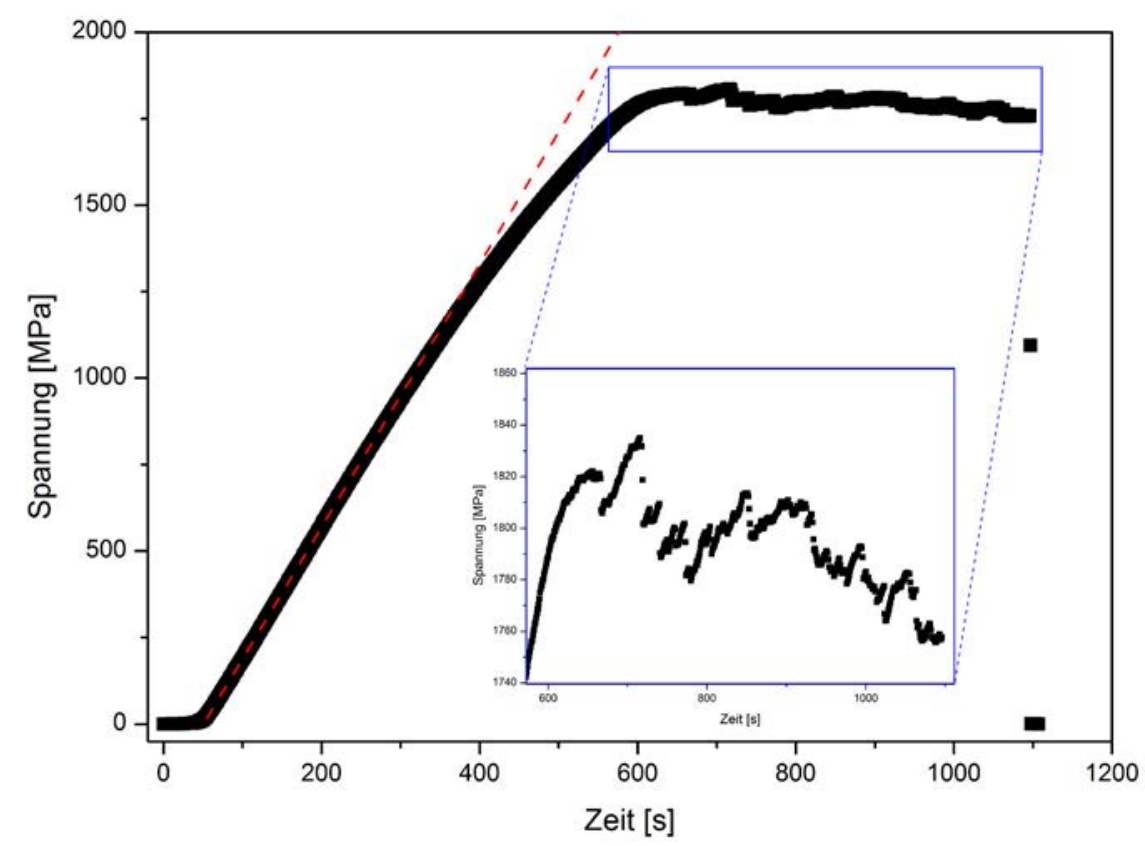

Abbildung 4.39.: Spannung gegen Zeit exemplarisch für AuNw25

Die Spannung lässt sich mit den Kraftdaten sowie der Querschnittsfläche über $\sigma=$ $F / A$ berechnen. Die Abtastrate der Kraft lag zwischen $1 \mathrm{~Hz}$ und $10 \mathrm{~Hz}$. In Abbildung 4.39 ist exemplarisch die Spannung gegen die Zeit von AuNw25 zu sehen. Hierbei wurde die Kraft mit einer Abtastrate von $1 \mathrm{~Hz}$ aufgenommen.

$\mathrm{Zu}$ Beginn des Tests verbleibt die Spannung zunächst auf dem Ausgangswert von $0 \mathrm{MPa}$, da hier der Draht erst gerade gezogen wird und somit noch nicht unter Zugspannung steht. Danach steigt die Spannung linear mit der Zeit an (siehe gestrichelte rote Linie in Abbildung 4.39). Dabei hat man es mit Steigungen zwischen $2 \mathrm{MPa} / \mathrm{s}$ und $8 \mathrm{MPa} / \mathrm{s}$ zu tun, je nach Aktuatorgeschwindigkeit. In den Tests, in denen die AktuatorSteuerung noch per Hand mit einer Schrittweite von 0,1V durchgeführt wurde, lassen sich im linearen Bereich die Sprünge in der Spannung für die einzelnen Schritte erkennen. Die Sprungweite liegt dabei bei circa 20MPa.

Danach weicht die Spannung nach und nach vom linearen Verhalten ab und die Steigung wird sukzessiv kleiner, wie sich an der Abweichung von der gestrichelten roten Linie in Abbildung 4.39 erkennen lässt. Typischerweise wie im Fall von AuNw25 wird eine solche Abweichung vom linearen Verhalten bereits ab einer Spannung von circa 900MPa erkennbar. Die Steigung knickt bei AuNw25 schließlich bei einer Span- 
nung von circa 1800MPa in einen horizontalen Verlauf ab. Dieses Abknicken verläuft bei AuNw25 eher kontinuierlich. Vor diesem Abknicken lassen sich noch keine Diskontinuitäten erkennen. Bei anderen Drähten tauchen bereits bei kleineren Spannungen kleine Sprünge auf, in denen die Spannung kurzzeitig nicht weiter ansteigt oder abnimmt. Desweiteren kann es bei anderen Drähten vor einem kompletten Abknicken in einen horizontalen Verlauf bereits zum Versagen des Drahtes kommen. Neben dem eher kontinuierlichem Abknicken wie bei AuNw25, kann der Übergang in einen horizontalen Verlauf eher abrupt vonstatten gehen. Typischerweise zeigen Drähte mit Zwillingsgrenze ein solches Verhalten, wobei das Abknicken hier mit einem mehr oder weniger großen Spannungsabfall einher geht.

Nach dem Übergang zu einem horizontalen Verlauf zeigt die Spannung mehrere diskrete Spannungsabfälle wie im Inset von Abbildung 4.39 zu sehen ist. Diese Spannungsabfälle betragen im Fall von AuNw25 bis zu 20MPa. Sie fallen im Allgemeinen bei Drähten mit Zwillingsgrenze größer aus und können hier bis zu 150MPa betragen. Nach einem Abfall steigt die Spannung in der Regel zunächst wieder an. Die Steigung hierbei ähnelt der Steigung im Anfangsbereich. Das allgemeine Spannungsniveau verläuft im Bereich zwischen 1750MPa und 1850MPa nahezu horizontal, wobei ein kleiner Abfall vom Übergang in den horizontalen Bereich bei 1820MPa bis hin zum Reißen bei $1755 \mathrm{MPa} z u$ beobachten ist.

\subsubsection{Spannungs-Dehnungs-Kurven}

Die Dehnungs- und Spannungswerte können nun zur Gewinnung der SpannungsDehnungs-Kurven verwendet werden. Die Korrelation zwischen Spannung und Dehnung erfolgt dabei üblicherweise über den Zeitpunkt des Reißens als Korrelationspunkt, da dieser Punkt in beiden Kurven eindeutig bestimmbar ist. Im Folgenden wird nun zunächst exemplarisch die Spannungs-Dehnungs-Kurve von AuNw25 vorgestellt und anhand dieser dann gezeigt, wie sich die verschiedenen Größen gewinnen lassen. Anschließend erfolgt die Vorstellung der Spannungs-Dehnungs-Kurven für die unterschiedlichen Proben- bzw. Versuchstypen.

Abbildung 4.40 zeigt exemplarisch die Spannungs-Dehnungs-Kurve für AuNw25. Nach dem Anfangsbereich, in dem der Draht gerade gezogen wird, steigt die Spannung zunächst linear mit der Dehnung an. In diesem Bereich, genauer zwischen 0,1\% und $0,75 \%$, wurde mit Hilfe einer linearen Regression das Elastizitätsmodul für den 


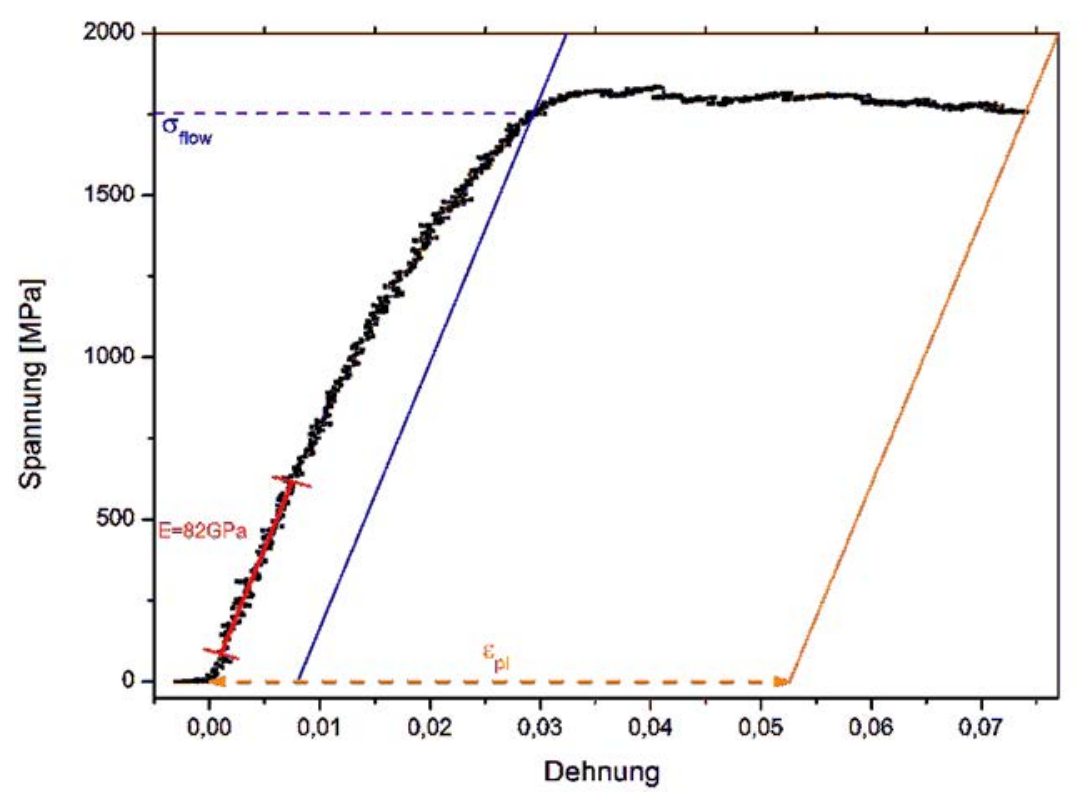

Abbildung 4.40.: Spannungs-Dehnungs-Kurve für AuNw25: Die rote Linie markiert den Bereich für den linearen Fit zur Bestimmung des E-Moduls; die blaue Linie dient der Bestimmung der Fließspannung; die orangefarbene Linie dient der Bestimmung der plastischen Dehnung beim Reißen.

Draht bestimmt. Die Festlegung der Grenzen erfolgte dabei so, dass zum einen keine Anfangseffekte wie zum Beispiel das Rutschen auf dem Untergrund nahe der Klebestellen im Zuge des Geradeziehens oder nichtlineare Effekte Berücksichtigung finden, aber zum anderen trotzdem ein ausreichend großer Bereich für die lineare Regression zur Verfügung steht. Für AuNw25 ergibt sich ein E-Modul von 82GPa.

Nach dem linearen Bereich nimmt die Steigung der Kurve sukzessiv ab und knickt schließlich bei einer Dehnung von circa 3\% und einer Spannung um 1800MPa in einen nahezu horizontalen Verlauf ab. Innerhalb dieses horizontalen Bereichs sind kleine Abfälle zu erkennen, allerdings sind diese durch die Korrelation von Spannung und Dehnung nicht mehr so deutlich auszumachen wie in der Spannung-Zeit-Kurve. Das Versagen des Drahtes ereignet sich schließlich bei einer Spannung von 1750MPa und einer Dehnung von $7,4 \%$.

Zur Bestimmung der Fließspannung wird eine Ausgleichsgerade mit dem E-Modul als Steigung und einer Offset-Dehnung von 0,8\% verwendet. Der Schnittpunkt mit der Spannungs-Dehnungs-Kurve stellt dann die jeweilige Fließspannung dar. Üblicherweise wird eine Offset-Dehnung von 0,2\% verwendet. Diese würde die Kurve aber in dem Bereich schneiden, in dem sich die Steigung augenscheinlich nur leicht vom linearen An- 
fangsbereich verringert hat und man somit nur eine nicht-lineare Elastizität evaluieren würde. Dieser Schnittpunkt trifft die Kurve gerade in dem Bereich, an dem die Kurve beginnt horizontal zu verlaufen. Der Wert für die Fließspannung liegt für AuNw25 bei $\sigma_{\text {flow }}=1753 \mathrm{MPa}$.

Weiterhin wird zur Bestimmung der plastischen Dehnung beim Versagen erneut eine Ausgleichsgerade mit dem E-Modul als Steigung an den letzten Punkt der Kurve angelegt. Der Schnittpunkt mit der Abszisse ergibt dann die plastische Dehnung. Diese wurde für AuNw25 mit $\epsilon_{p l}=5,26 \%$ bestimmt.

\section{Einkristalline Drähte}

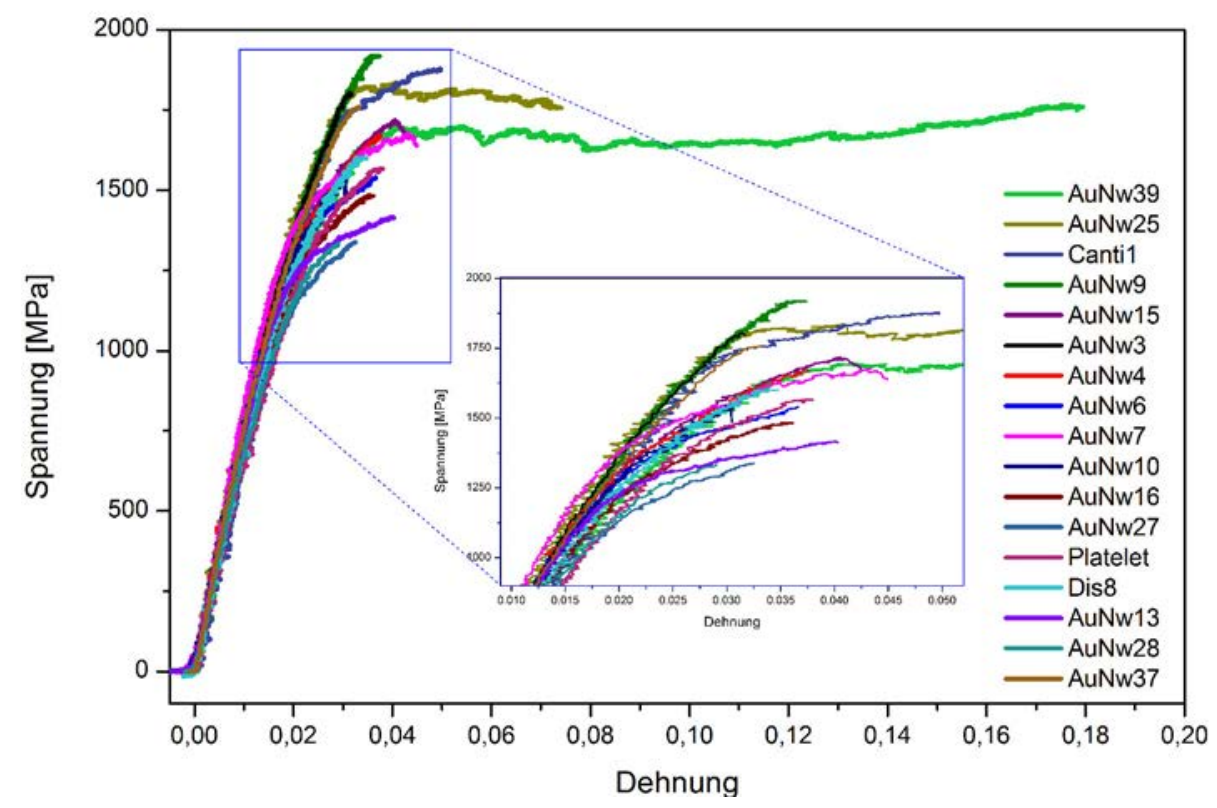

Abbildung 4.41.: Spannungs-Dehnungs-Kurven der einkristallinen Drähte. Das Inset zeigt den markierten Bereich in einer vergrößerten Darstellung.

Abbildung 4.41 zeigt die Spannungs-Dehnungs-Kurven für einkristalline Drähte. Alle Drähte zeigen zunächst einen ziemlich linearen Anstieg der Spannung bis zu einer Dehnung von circa 1\%. Die Steigung und damit das E-Modul variiert zwischen den einzelnen Drähten vergleichsweise stark. So wurden Werte für das E-Modul zwischen 66,5GPa und 82GPa ermittelt. Nach dem linearen Anfangsbereich nimmt die Steigung der Kurven sukzessiv ab. Das Versagen der Drähte erfolgt für die meisten Drähte nach einer plastischen Dehnung im Bereich zwischen 0,9\% bis 2,7\%. Einen ausgeprägteren 
plastischen Bereich zeigen nur zwei Drähte: AuNw25 und AuNw39. AuNw25 weist beim Versagen eine plastische Dehnung von 5,3\% auf und AuNw39 sogar eine plastische Dehnung von $15,4 \%$.

\section{Blindversuche}

Um den Einfluss des Elektronenstrahls im REM zu untersuchen, wurden 4 Drähte getestet, ohne sie dabei direkt mit dem Elektronenstrahl abzulichten. Um dennoch eine Dehnungsanalyse zu erhalten, wurde zunächst die Messlänge der Drähte bestimmt und der Elektronenstrahl danach zu Seite gelenkt, so dass sich nicht mehr der Draht, jedoch der Kraftsensor und das TEM-Grid im Blickfenster befanden. Der Test verlief danach wie üblich. Für die Dehnungsanalyse mittels DIC wurde schließlich jeweils ein markanter Punkt auf dem Sensor und dem Grid verfolgt. Abbildung 4.42 zeigt die 4 Spannungs-Dehnungs-Kurven dieser so getesteten Drähte. Die Kurven zeigen zunächst

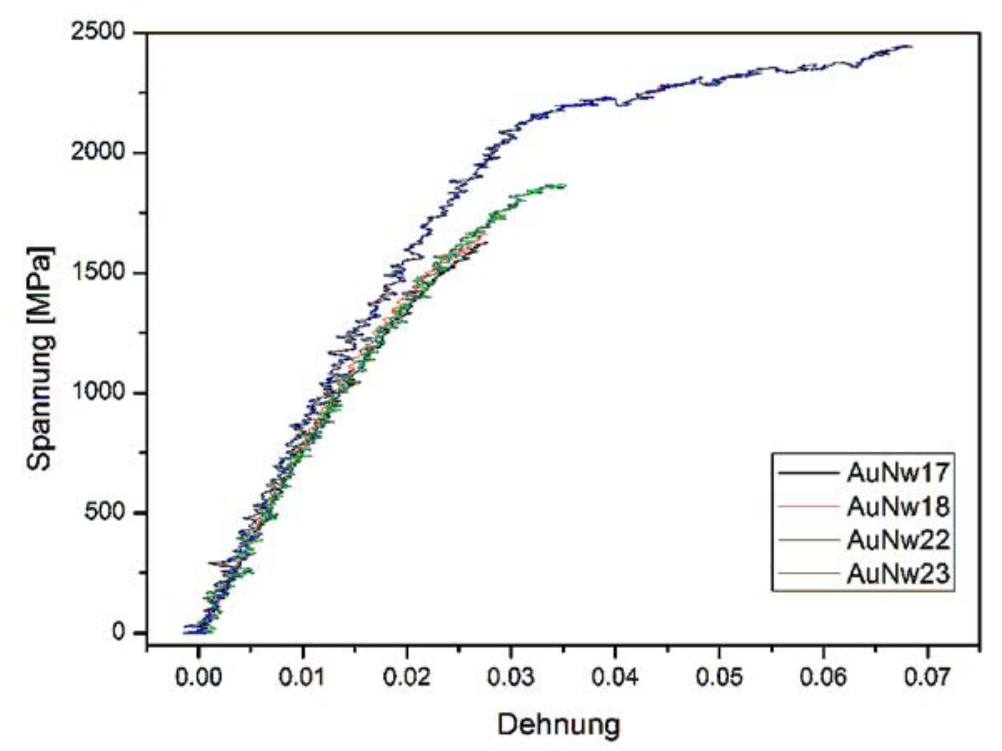

Abbildung 4.42.: Spannungs-Dehnungs-Kurven der Drähte, die ohne direkte Belichtung durch den Elektronenstrahl gezogen wurden

erneut einen linearen Anstieg. Die Steigungen und damit das E-Modul liegen zwischen 74GPa und 85GPa. Der Mittelwert liegt bei 79,7GPa und fällt damit im Vergleich zu den herkömmlichen Tests mit Elektronenstrahl etwas höher aus. Nach dem linearen Anstieg nimmt die Steigung der Kurven erneut sukzessiv ab. Dabei unterscheidet 
sich das Verhalten von AuNw23 von den drei übrigen Drähten. So verringert sich bei AuNw23 die Steigung nur sehr langsam und erst bei einer hohen Spannung von über 2000MPa ist ein deutlicher Knick zu erkennen mit anschließender plastischer Verformung bis zum Reißen bei 3,9\%. Bei den drei anderen Drähten nimmt die Steigung kontinuierlicher ab. Ein ausgeprägter Knick sowie eine ausgeprägte plastische Dehnung sind nicht feststellbar.

\section{Drähte mit Versetzungen}

Insgesamt wurden 3 Drähte im REM getestet, bei denen vorher eine Beanspruchung wie in Kapitel 3.4 beschrieben erfolgte und Versetzungen in den Draht eingebracht wurden - jeweils bei allen drei durch Biegen bzw. Quetschen. AuDis5 (siehe Abbildung 4.43 (a)) zeigt einen stark gequetschten Bereich. In diesem Bereich befinden sich viele Kontraste von Versetzungen. AuDis6 (Abbildung 4.43 (b)) zeigt nur einen linienhaften Versetzungskontrast in der Mitte der Drahtlänge. AuDis7 (Abbildung 4.43 (c)) weist eine verjüngte Stelle mit Versetzungen auf.

Abbildung 4.44 zeigt die Spannungs-Dehnungs-Kurven dieser Drähte. Die Kurven liegen bis zum jeweiligen Reißen der Drähte sehr dicht übereinander. Dabei lassen die Kurven zunächst wieder einen linearen Anstieg erkennen. Das E-Modul im Bereich zwischen 0,1\% Dehnung und 0,75\% Dehnung liegt zwischen 82,0GPa und 85,5GPa und damit am oberen Ende bzw. über den Werten herkömmlicher Proben und auch über dem theoretischen Wert. Nach dem linearen Anstieg nimmt die Steigung der Kurven sukzessiv bis zum Reißen ab. Dabei kommt es aber in keinem Fall zu einem horizontalen Verlauf. Die Spannungen und Dehnungen beim Reißen der Drähte unterscheiden sich ziemlich stark voneinander. So versagt AuDis5 bereits bei einer plastischen Dehnung von 0,23\% und einer Spannung von 1272MPa, was aufgrund des stark deformierten Bereichs durch die Beanspruchung vor dem Versuch nicht weiter verwunderlich ist, so dass für die weitere Auswertung dieser Draht herausgenommen wird. AuDis6 versagt bei einer plastischen Dehnung von 1,32\% und einer Spannung von 1763MPa, AuDis7 bei einer plastischen Dehnung von 3,03\% und einer Spannung von 2083MPa.

Nach dem Versuch war in der Mikrostruktur von AuDis6 und AuDis7 ebenfalls die Speicherung von planaren Defekten entlang des Drahtes erkennbar und damit ergab sich kein Unterschied zu Drähten ohne gespeicherte Versetzungen vor dem Versuche, so dass es auch hier keine Hinweise auf einen unterschiedlichen Verformungsmechanismus 


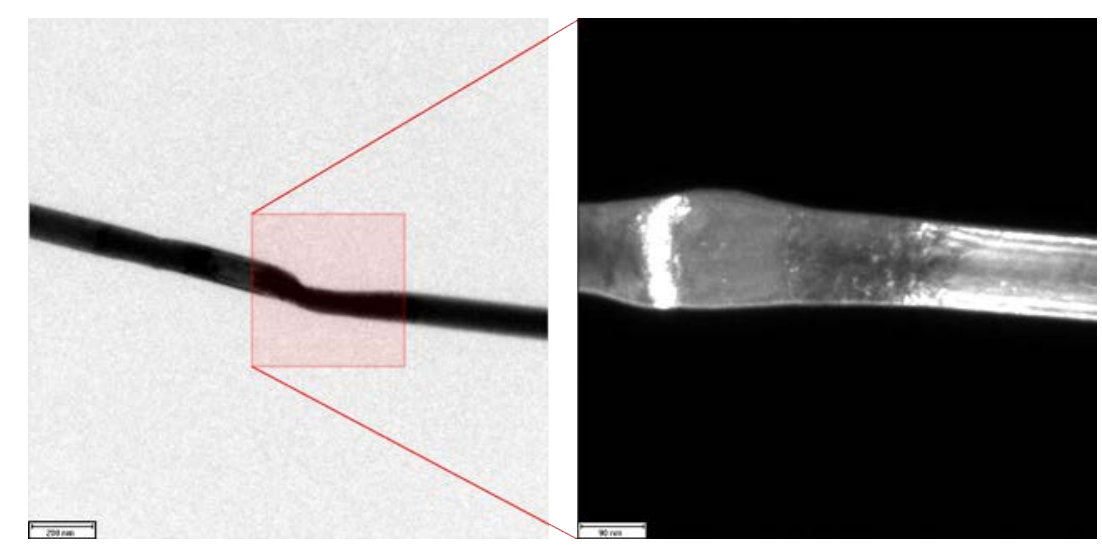

(a)

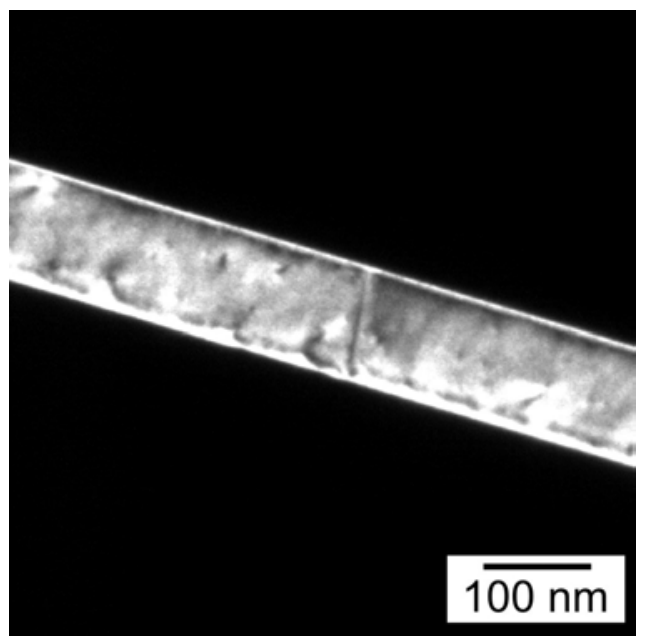

(b)

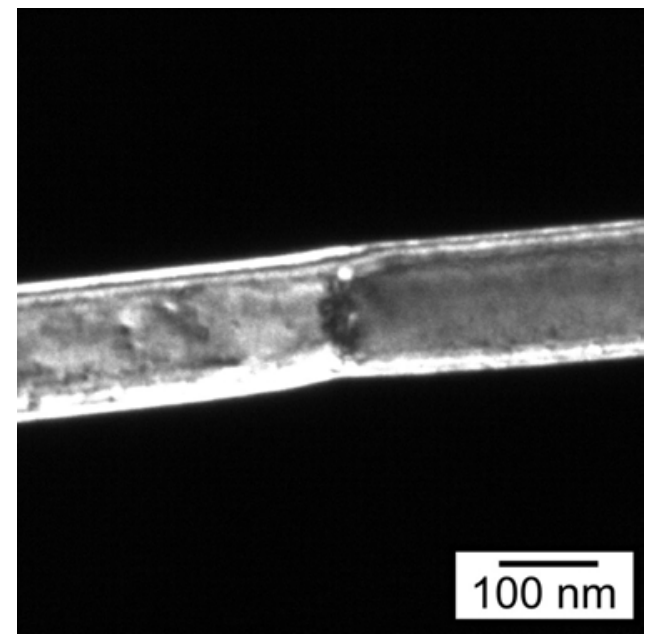

(c)

Abbildung 4.43.: TEM-Aufnahmen der Drähte nach der Vorverformung und vor dem Test: a) AuDis5, b) AuDis6 und c) AuDis7 


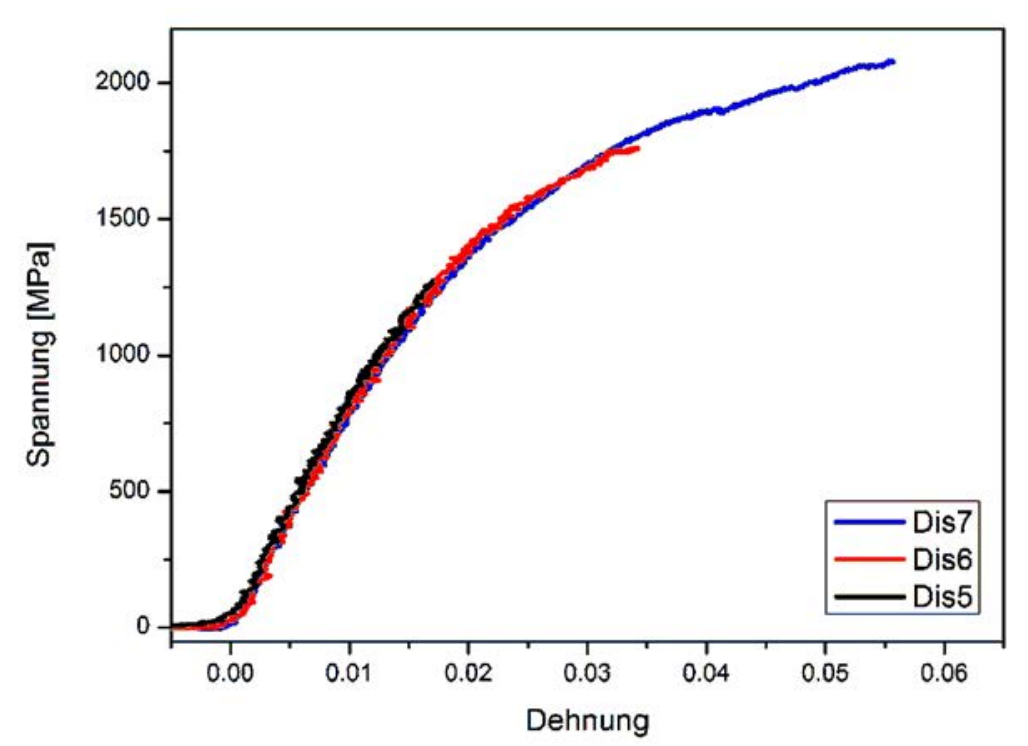

Abbildung 4.44.: Spannungs-Dehnungs-Kurven der Drähte, in die durch Vorverformung Versetzungen eingebracht wurden

gibt, wie bei den beanspruchten Drähten, die im TEM getestet wurden. Überraschenderweise konnte in AuDis5 trotz der starken Querschnittsveränderung zumindest ein planarer Defekt entlang des Drahtes ausgemacht werden, so dass es selbst hier zu einem Nukleationsevent an einer Drahtstelle gekommen ist, die entfernt von dem stark deformierten Bereich lag.

\section{Drähte getestet in H2O-Atmosphäre}

Zwei Drähte wurden im REM durch das Einlassen von Wasserdampf im Niedrigvakuum mit einem Kammerdruck von 0,5mbar getestet. Durch das Einlassen des Wasserdampfes war es erforderlich, eine zusätzliche Blende unterhalb des Polschuhs sowie einen anderen Detektor zu verwenden, so dass die Bildqualität während der Versuche stark reduziert war. So ist das Rauschen in den Dehnungswerten entsprechend größer als unter normalen Bedingungen. Die Spannungs-Dehnungs-Kurven sind in Abbildung 4.45 zu sehen. Die Kurven steigen zunächst wieder ziemlich linear an. Das E-Modul liegt hier mit 72,5GPa (H2O 1) und 62,9GPa (H2O 4) am unteren Ende im Vergleich zu den anderen getesteten Drähten. Nach dem linearen Anstieg nimmt die Steigung sukzessiv ab. Dabei findet der Übergang vom linearen Anfangsbereich zu einem fast horizontalen Verlauf der Kurven in einem relativ kleinen Dehnungsintervall statt. Dieser plastische 
Bereich ist jedoch für beide Drähte nicht besonders stark ausgeprägt. H2O 1 reißt bei einer plastischen Dehnung von 1,72\% und einer Spannung von 1338MPa, H2O 4 bei einer plastischen Dehnung von 0,91\% und einer Spannung von 1315MPa.

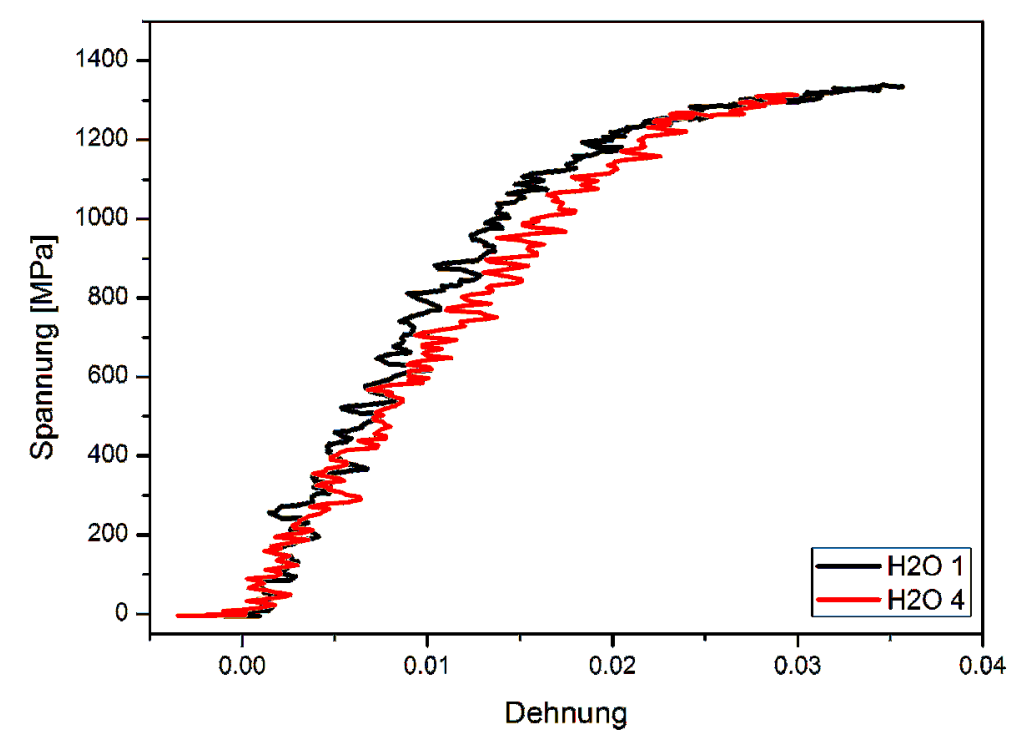

Abbildung 4.45.: Spannungs-Dehnungs-Kurven der Drähte, die bei 0,5mbar getestet wurden

\section{Abweichendes Spannungs-Dehnungs-Verhalten}

Für einen der getesteten Drähte ergab sich ein stark abweichendes SpannungsDehnungs-Verhalten. Bei diesem Draht stellte sich nachträglich heraus, dass es im Zuge des Transferprozesses lediglich seitlich des Drahtes zu einer schwachen Verbindung zum Untergrund durch das Platin gekommen ist, wie Abbildung 4.46 (a) zeigt. Die Spannungs-Dehnungs-Kurve dieses Drahtes in Abbildung 4.46 (b) zeigt zunächst den üblichen Anstieg der Spannung mit zunehmender Dehnung. Dabei kommt es zu einer geringen Rotation der Platinbefestigung wie im 2ten REM-Bild von Abbildung 4.46 (c) durch den roten Pfeil verdeutlicht wird. Beachtlich ist, dass trotz dieser teilweise schwachen Befestigung das E-Modul zu 73,3GPa bestimmt wurde und somit nur leicht unterhalb des Mittelwerts der restlichen Drähte liegt. Bei einer Dehnung von 2, 2\% und einer Spannung von 1,3GPa kommt es dann schlagartig zu einem Spannungsabfall auf circa 200MPa zusammen mit einem Sprung in der Dehnung von 1,7\%. In den 


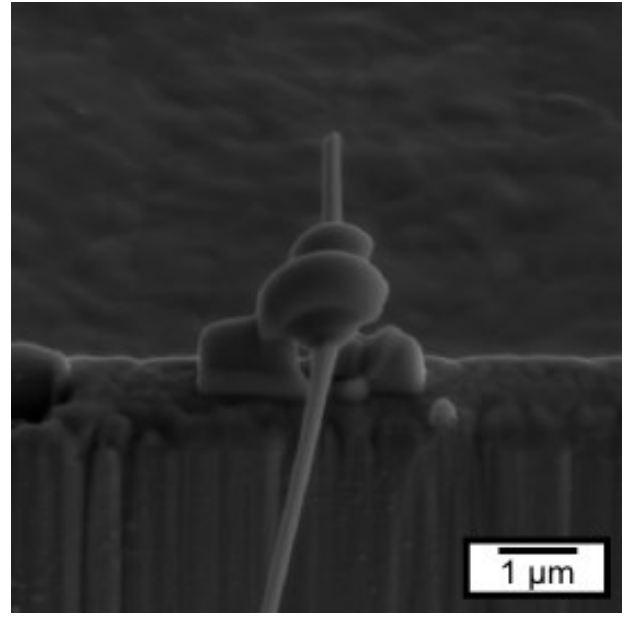

(a)
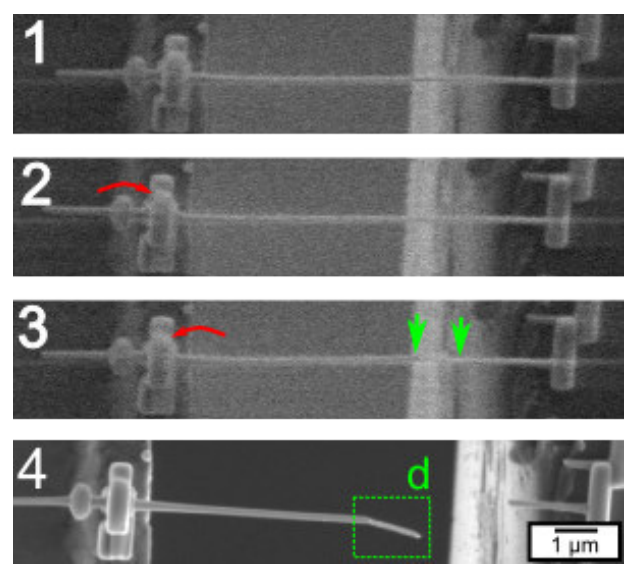

(c)

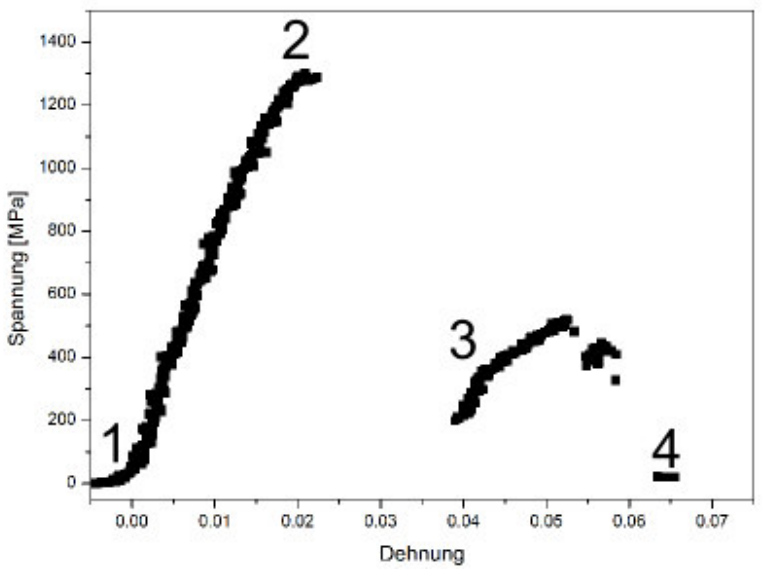

(b)

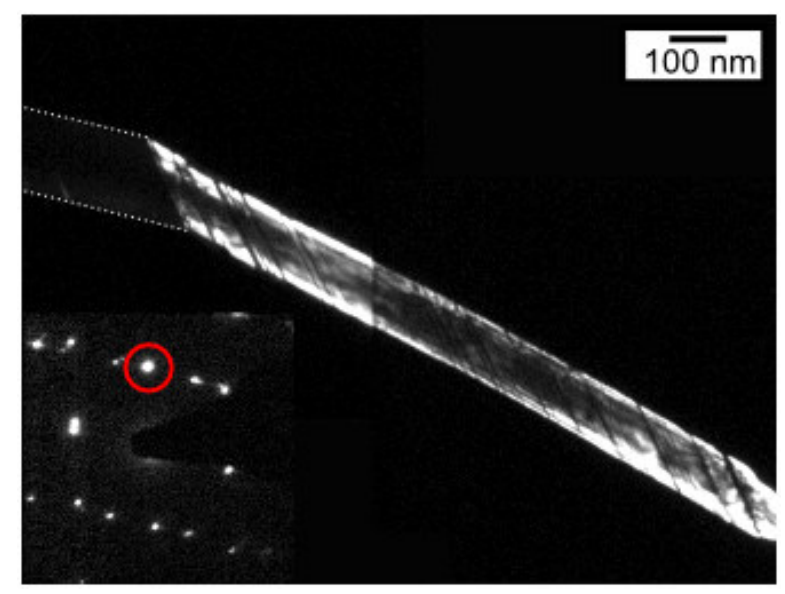

(d)

Abbildung 4.46.: Abweichendes Spannungs-Dehnungs-Verhalten: (a) zeigt die REMAufnahme einer unvollkommenen Klebestelle; ein Kontakt zum Untergrund ist nur auf einer Seite des Drahtes gegeben; (b) zeigt die zugehörige Spannungs-Dehnungs-Kurve; (c) zeigt insgesamt vier REMBilder während und nach dem Test; der Zeitpunkt ist jeweils (b) durch die jeweilige Nummer markiert; (d) TEM-Dunkelfeld-Aufnahme des markierten Bereiches von Bild 4 in (c); das Inset zeigt das zugehörigen Beugungsbild mit dem zur Abbildung verwendeten Reflex 
REM-Aufnahmen (Bild 3) ist erkennbar, dass zum einen die Platinbefestigung wieder in Richtung der Ausgangsposition rotiert ist. Zum anderen hat sich der Draht in dem circa 900nm breiten Bereich zwischen den grünen Pfeilen stark verformt.

Die weitere Verformung des Drahtes erfolgt bei Spannungen zwischen 200MPa und 520MPa, wobei die Spannung zunächst kontinuierlich ansteigt bis sie bei circa 5, 5\% Dehnung einen zweiten, kleinen Abfall zeigt. Das Versagen erfolgt schließlich bei einer Dehnung von 5,8\% an der rechten Grenze des stark verformten Bereiches. Bis zum Versagen wächst der Bereich zwischen den beiden grünen Pfeilen auf eine Breite

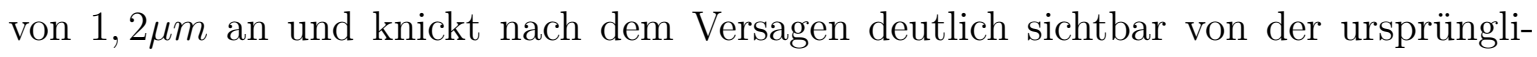
chen Orientierung des Drahtes ab, wie in REM-Bild 4 und ebenfalls in dem TEMDunkelfeldaufnahmen in 4.46 (d) zu sehen ist. Die Oberfläche der ursprüngliche Drahtorientierung ist zur Verdeutlichung gestrichelt nachgezogen. Die TEM-Aufnahmen zusammen mit dem Beugungsbild zeigen, dass es sich bei dem stark verformten Bereich um einen Zwilling handelt. Zu beachten ist, dass entlang des verzwillingten Bereiches dunkle Bereiche der ursprünglichen Drahtorientierung zu sehen sind. In keinem anderen Fall konnte ein derartiger Spannungsabfall oder auch das sprunghafte Ausbilden eines breiten verzwillingten Bereiches beobachtet werden. So scheint die Platinbefestigung und die Möglichkeit der Rotation dieser Befestigung diese Verformung zu verursachen.

\section{Drähte mit Zwillingsgrenze}

Insgesamt wurden 6 Drähte mit Zwillingsgrenze in normaler Abfolge im REM getestet. Die Spannungs-Dehnungs-Kurven dieser Drähte sind in Abbildung 4.47 dargestellt.

Die Drähte mit Zwillingsgrenze zeigen ebenfalls einen linearen Anfangsbereich, wobei die Werte für das Elastizitätsmodul hier zwischen 62,3GPa und 80,3GPa liegen. Nach linearem Anfangsbereich nimmt die Steigung der Kurven zunächst sehr langsam ab, gefolgt von einem deutlichen Abknicken. Das Abknicken geschieht bei unterschiedlichen Spannungen zwischen 1200MPa und 1800MPa. Die vier Kurven, bei denen die Spannung beim Abknicken am höchsten ist, weisen eine wiederkehrende Folge von kleinen Spannungsabfällen gefolgt von kleinen Spannungsanstiegen auf. Dabei sind die jeweiligen Spannungsabfälle direkt nach dem Abknicken scheinbar am größten. Diese werden mit zunehmender Dehnung kleiner. Das allgemeine Spannungsniveau scheint aber eher konstant zu verlaufen. Die beiden Kurven, die bereits bei kleineren Spannungen abknicken, zeigen ebenfalls Spannungsabfälle gefolgt von Spannungsanstiegen. 


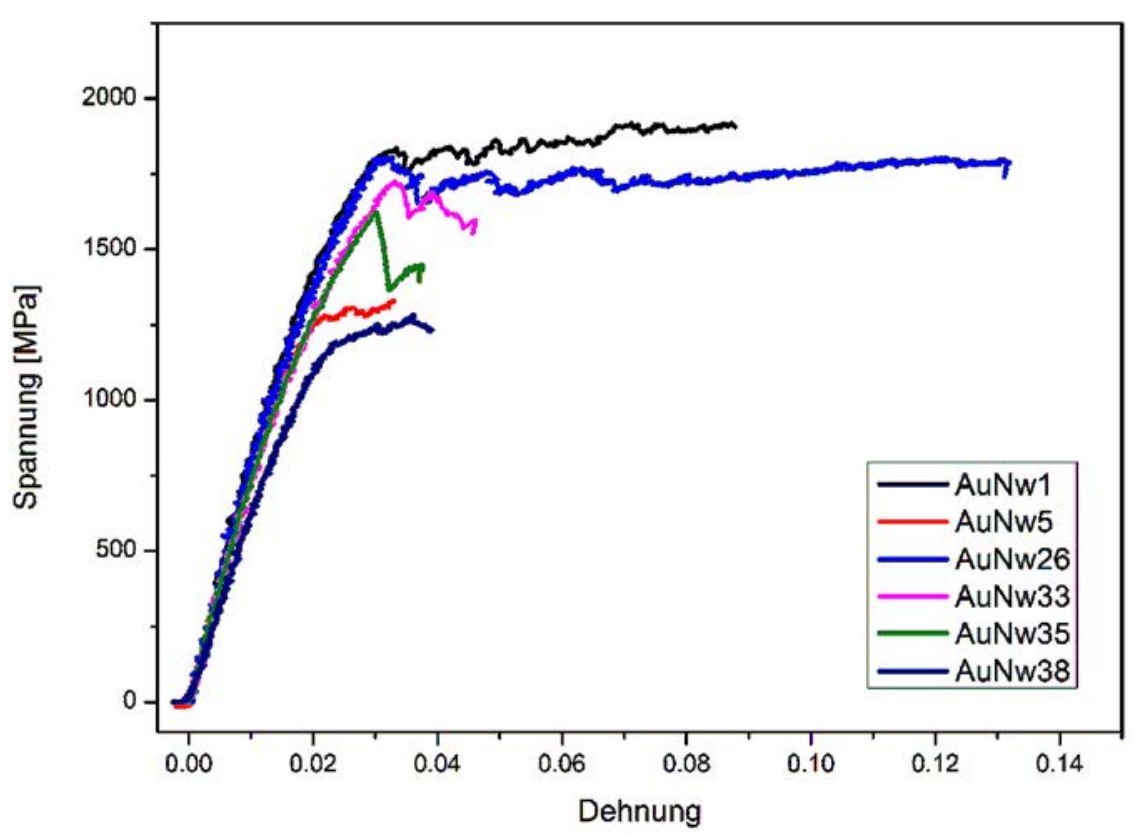

Abbildung 4.47.: Spannungs-Dehnungs-Kurven der Drähte mit Zwillingsgrenzen

Diese sind jedoch nicht so stark ausgeprägt wie bei den übrigen Drähten. Das Versagen der Drähte erfolgt schließlich nach einer Dehnung zwischen 3,2\% und und 13, $2 \%$.

\section{Entlastungsversuche}

Insgesamt wurden bei 5 einkristallinen Drähten und 2 Drähten mit Zwillingsgrenze Entlastungsversuche durchgeführt. Hierbei wird der Draht zunächst bis zu einem bestimmten Punkt gedehnt. Bei der Entladung wird die Ausdehnung des Aktuators mit der gleichen Geschwindigkeit wie beim Beladen zurückgefahren bis eine leichte Biegung des Drahtes im REM-Bild zu erkennen ist. Anschließend erfolgt eine erneute Dehnung des Drahtes, wobei mit jedem neuen Beladungsschritt eine Erhöhung der maximalen Dehnung stattfand. Die Zahl der Entlastungssegmente variiert zwischen eins (AuNw2, AuNw34) und zwölf (AuNw30). Da keine in-situ-Dehnungsmessung möglich ist, ist der Abstand zwischen den einzelnen Segmenten dabei nicht gleich.

Abbildung 4.48 (a) zeigt die Spannungs-Dehnungs-Kurven der Entlastungsversuche für die einkristallinen Drähte. Zur Veranschaulichung sind in Abbildung 4.48 (b) sämtliche Beladungsschritte und in Abbildung 4.48 (c) sämtliche Entladungsschritte von AuNw30 separat dargestellt.

Die ersten Beladungskurven bis zu einer Dehnung von circa 0,75\% zeigen einen 


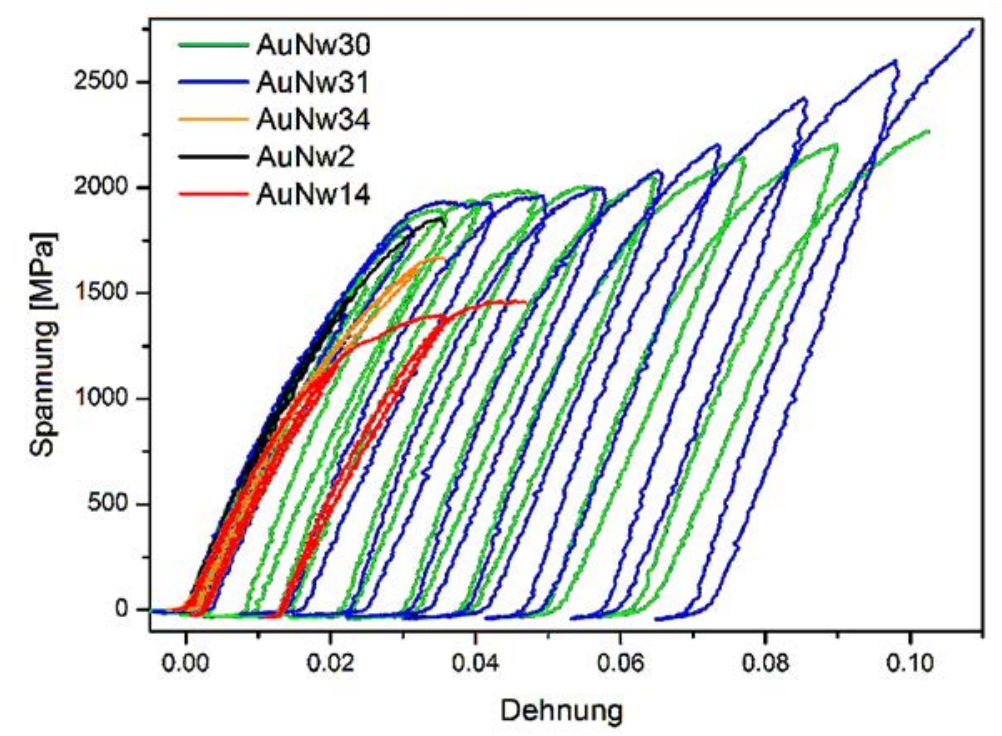

(a)

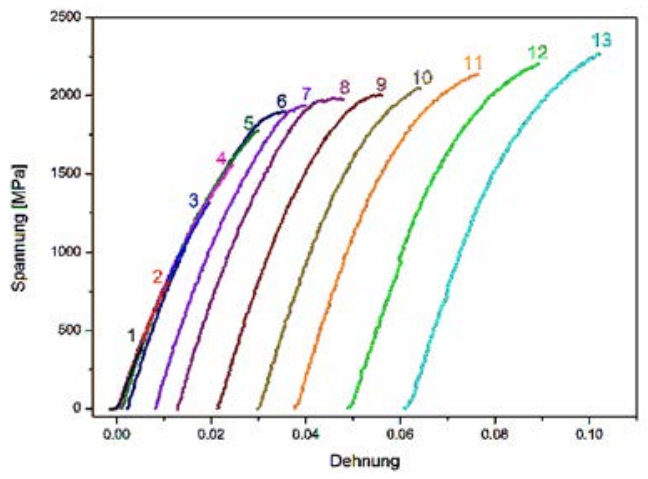

(b)

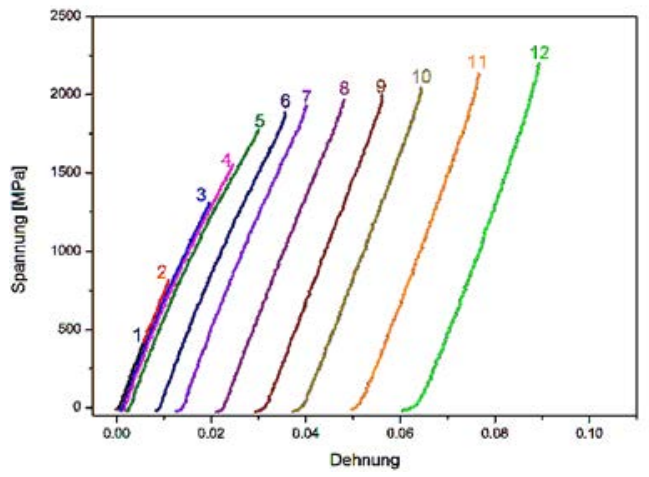

(c)

Abbildung 4.48.: Spannungs-Dehnungs-Kurven der Entlastungsversuche von einkristallinen Drähten: (a) Spannungs-Dehnungs-Kurven aller Entlastungsversuche, (b) Beladungskurven von AuNw30 und (c) Entlastungskurven von $\mathrm{AuNw} 30$ 
linearen Anstieg. Die Steigungen und die in dem Bereich von 0, 1\% bis 0,75\% ermittelten E-Moduln variieren hierbei zwischen 70GPa (AuNw14 und AuNw34) und 83,5GPa (AuNw31). Nach dem linearen Anstieg nimmt die Steigung der Beladungskurven ab einer Dehnung in einem Bereich von etwas unterhalb von 1\%-Dehnung ab, bevor es zum eigentlichen 'Abknicken' der Kurven kommt. Das Abknicken der Kurven erfolgt dabei analog zu den normalen Versuchen bei unterschiedlichen Spannungen. So knickt AuNw14 bereits bei einer Spannung von circa 1250MPa, AuNw34 bei einer Spannung von 1650MPa und die restlichen drei Drähte bei einer Spannung von über $1800 \mathrm{MPa}$ (Beladungskurve 6 für AuNw30 in Abbildung 4.48 (b)).

Entladesegmente aus dem Bereich vor dem Abknicken der Kurven führen zu einem Rückgang der Kurven in die Nähe des Ursprungs bei einer Spannung von 0MPa und einer bleibenden plastischen Dehnung von bis zu 0,3\%. Je weiter die vorhergehende Dehnung im Beladungssegment war, desto höher ist dabei die bleibende Dehnung. AuNw30 wurde bis zum Abknicken der Kurve insgesamt 5mal entlastet (siehe Abbildung 4.48 (c). Hier steigt die bleibende Dehnung von weniger als 0,1\% in den ersten beiden Entlastungskurven bis ungefähr 0,25\% im fünften Entlastungssegment.

Nach dem Abknicken der Kurven kommt es bei AuNw2 und und AuNw34 direkt zum Versagen des Drahtes, während die restlichen drei Drähte einen plastischen Bereich zeigen, der bei AuNw30 und AuNw31 recht stark ausgeprägt ist (max. Dehnung bei 10,3\% bzw. 10,9\%). Entladungssegmente aus diesem plastischen Bereich nach dem Abknicken der Kurven hinterlassen eine deutlich größere bleibende Dehnung beim Erreichen der Ausgangsspannung von 0MPa. So nimmt die bleibende Dehnung von AuNw30 von 0,9\% in Entlastungschritt 6 bis zu 6,2\% in Entlastungsschritt 12 mit jedem Zyklus zu.

Betrachtet man die Form der Entladekurven, so ist eine fortlaufende Veränderung mit jedem neuen Zyklus erkennbar. Die ersten Entladekurven zeigen ähnlich wie die Beladungskurven oberhalb von etwa $1 \%$ ein nichtlineares Verhalten, d.h. mit Beginn der Entlastung nimmt die Spannung zunächst weniger stark ab. Für Entladekurven nach dem 'Abknicken' (ab Entladekurve 6 für AuNw30) nimmt dieser Effekt immer mehr ab. Es ergibt sich stattdessen mit Beginn der Entlastung zunächst eine stärkere Abnahme der Spannung. Evaluiert man das E-Modul für jede Entladekurve zwischen $80 \mathrm{MPa}$ und 600MPa (entspricht dem Dehnungsintervall von 0,1\% bis 0,75\% zur Bestimmung des E-Moduls aus dem ersten Beladungsschritt), bemerkt man eine gewisse Streuung, 
jedoch keinen Trend mit zunehmender bleibender Dehnung.

Die Beladungskurven stimmen in der Regel nicht mit der vorherigen Entladungskurve überein, sondern zeigen eine Art Hysterese, wobei die Spannung zum einen bei etwas kleineren Dehnungen bereits wieder zu steigen beginnt und zum anderen die Steigung größer ist als bei der vorherigen Entladekurve. Diese Hysterese scheint mit wachsender Dehnung zuzunehmen, d.h. dass der Dehnungsunterschied zwischen Ende der Entlastung und Beginn der Belastung größer wird. Weiterhin ist die Hysterese bei den verschiedenen Drähten unterschiedlich stark ausgeprägt. So zeigen AuNw30 und AuNw31 eine starke Hysterese, während die übrigen drei Drähte eher eine kleine Hysterese aufweisen oder wie bei AuNw2, für den überhaupt keine Hysterese auszumachen ist, wobei in diesem Fall die Entlastung nicht bis zum leichten Biegen durchgeführt wurde, sondern bis zu einer Restspannung von etwas mehr als 200MPa.

Bei genauer Analyse des Verlaufs der Beladungskurven ist erkennbar, dass sie bis zum 'Abknicken' die Beladungskurven gut übereinanderliegen, wobei mit jedem Beladungsschritt die maximale Spannung wächst. Hierbei fällt jedoch auf, dass es trotz bleibender Dehnung bei der vorherigen Entlastung zu einem Überschneiden der Kurven kommt (siehe Beladungskurve 5 und 6 von AuNw30). Dies liegt daran, dass die Steigung der Beladungskurve mit jedem Zyklus zunimmt. Evaluiert man erneut die Steigung für jede Beladungskurve zwischen 80MPa und 600MPa, so erhält man im Fall von AuNw30 eine stetige Zunahme von 80,3GPa für den ersten Beladungsschritt bis zu 99,2GPa in Beladungsschritt 11. Die beiden letzten Beladungskurven zeigen dann wieder leicht kleinere Steigungen. Weiterhin scheint sich die Form der Beladungskurven nach dem 'Abknicken' erneut zu verändern. Während man in den Beladunskurven 6 bis 8 jeweils ein Abknicken erkennen kann und die Beladungskurven jeweils fast an den letzten Punkt der vorherigen Kurve zurückreichen, nimmt die Steigung ab Beladungskurve 9 homogener ab, ohne dass ein deutliches Abknicken und gleichzeitig ein deutlicher Versatz zur vorherigen Kurve auftreten, wobei die maximale Spannung mit jedem Zyklus wächst.

Abbildung 4.49 zeigt die beiden Entlastungskurven für die Drähte mit Zwillingsgrenze. Die beiden Kurven zeigen ebenfalls einen linearen Anstieg bis zu einer Dehnung von circa 0,75\%. Das E-Modul in diesem Bereich ausgewertet ergibt 76,8GPa für AuNw32 und 76,2GPa für AuNw36. Hinter dem linearen Bereich nimmt die Steigung in beiden Kurven langsam ab. Das 'Abknicken' verbunden mit einem kleinen Span- 


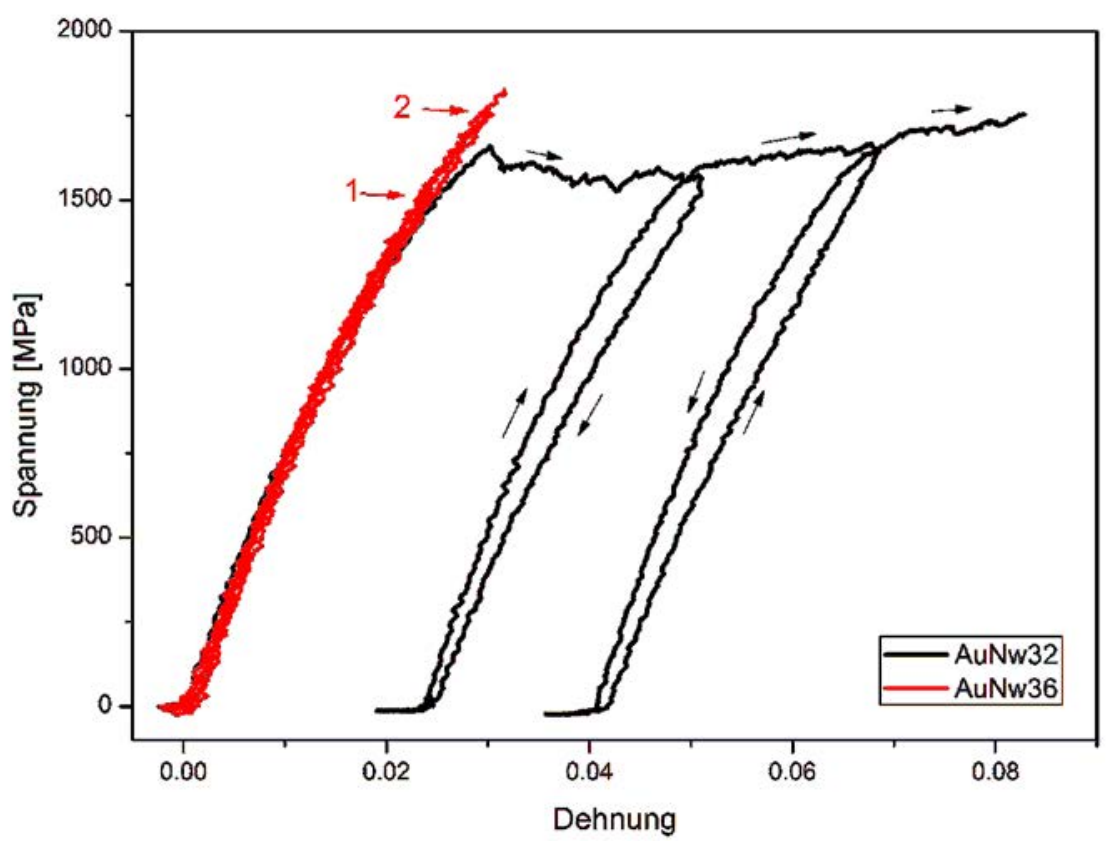

Abbildung 4.49.: Spannungs-Dehnungs-Kurven der Entlastungsversuche von Drähten mit Zwillingsgrenze

nungsabfall erfolgt bei AuNw32 bei einer Dehnung von 3, $0 \%$ und einer Spannung von 1660MPa. Bei AuNw36 stellt das 'Abknicken' gleichzeitig das Versagen des Drahtes dar und erfolgt bei einer Dehnung von 3,2\% und einer Spannung von 1820MPa.

Vor dem Abknicken bzw. Reißen wurden zwei Entladevorgänge für AuNw36 durchgeführt. Das erste Entladesegment beginnt bei einer Dehnung von 2,4\% und einer Spannung von 1520MPa, das zweite Entladesegment bei einer Dehnung von 3,0\% und einer Spannung von 1750MPa (in Abbildung 4.49 durch die roten Pfeile bei '1' und '2' markiert). Beide Entladekurven zeigen dabei einen ähnlichen nichtlinearen Verlauf wie die Beladungskurven zurück zum Ausgangspunkt, wobei Dehnungen im Bereich von 0,1\% zurückbleiben. Die folgenden Beladungskurven zeigen auch hier eine kleine Hysterese und können die vorhergehenden Beladungskurven schneiden.

Nach dem Abknicken verläuft die Kurve von AuNw32 überwiegend horizontal mit vielen kleinen Spannungsabfällen und -anstiegen. In diesem Bereich wurde AuNw32 zwei mal entlastet. Das erste Entladesegment fängt bei einer Dehnung von 5, $1 \%$ bei einer Spannung von 1560MPa an und führt zu einer bleibenden Dehnung von circa 2, $4 \%$ bei kompletter Entlastung. Das zweite Entlastungssegment beginnt bei einer Dehnung von 6,9\% und einer Spannung von 1650MPa und führt bei kompletter Entlastung zu einer bleibenden Dehnung von circa 4,2\%. Beide Entladekurven zeigen dabei ein leicht 
nichtlineares Verhalten, d.h. die Spannung fällt auch hier zu Beginn der Entladung schwächer ab. Die folgende Belastung beginnt erneut bei etwas kleineren Dehnungen als bei den bleibenden Dehnungen der vorhergehenden Entlastung. Weiterhin ist die Steigung der Beladungskurven erneut größer, so dass wieder eine Hysterese erkennbar ist. Die Beladungskurven schneiden schließlich die vorhergehende Beladungskurve und knicken bei etwa der gleichen Spannung ab, bei der die vorhergehende Entladung begonnen hat, wobei es hierbei nicht zu einem nennenswerten Spannungsabfall kommt.

\subsubsection{Ergebnisse der Spannungs-Dehnungs-Kurven}

Tabelle 4.4 fasst die wichtigsten Größen der Drähte zusammen. Hierzu gehören die Besonderheiten des jeweiligen Drahtes, der Durchmesser $d$, das E-Modul E, die Fließspannung bei 0,2\%-Offset-Dehnung $\sigma_{0,2 \%}$ und die plastische Dehnung $\epsilon_{p l}$.

\section{E-Modul}

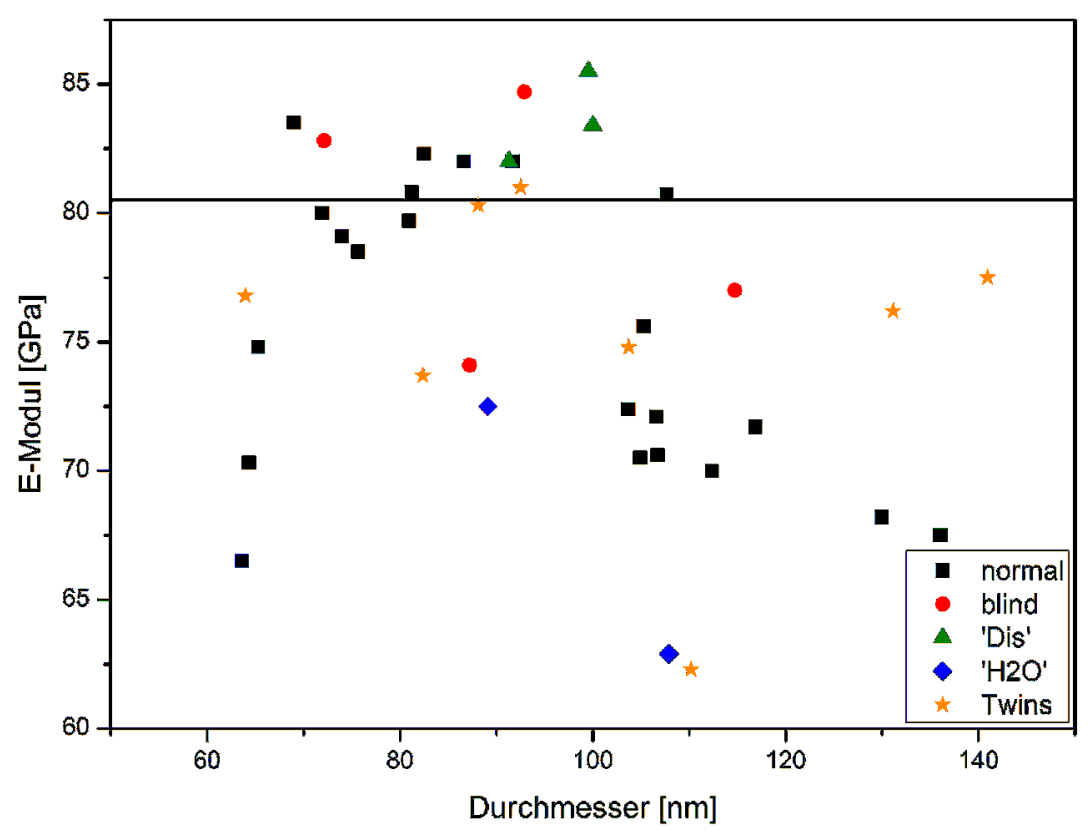

Abbildung 4.50.: E-Modul gegen den Durchmesser: Die horizontale Linie kennzeichnet den theoretischen Wert von $80,5 \mathrm{GPa}$ für Gold in $<110>$-Richtung.

Das E-Modul wurde für alle Drähte in dem Bereich zwischen 0,1\% und 0,75\% Dehnung ausgewertet. Dabei ergibt sich über alle Drähte gesehen eine große Streuung zwischen 62GPa und 86GPa. Der Mittelwert liegt bei 76GPa, was etwas unterhalb des 


\begin{tabular}{|c|c|c|c|c|c|}
\hline Draht & & $\mathrm{d}[\mathrm{nm}]$ & $\mathrm{E}[G P a]$ & $\sigma_{\text {flow }}[M P a]$ & $\epsilon_{p l}[\%]$ \\
\hline AuNw2 & Entl. & 81 & 80,8 & 1726 & 1,24 \\
\hline AuNw3 & & 81 & 79,7 & 1771 & 0,91 \\
\hline $\mathrm{AuNw} 4$ & & 74 & 79,1 & 1475 & 1,64 \\
\hline AuNw6 & & 108 & 80,7 & 1419 & 1,75 \\
\hline $\mathrm{AuNw} 7$ & & 83 & 82,3 & 1505 & 2,49 \\
\hline AuNw9 & & 65 & 74,8 & 1831 & 1,15 \\
\hline $\mathrm{AuNw} 10$ & & 107 & 72,1 & 1514 & 0,98 \\
\hline $\mathrm{AuNw} 13$ & & 105 & 75,6 & 1295 & 2,18 \\
\hline $\mathrm{AuNw} 14$ & Entl. & 107 & 70,6 & 1290 & 2,66 \\
\hline $\mathrm{AuNw} 15$ & & 76 & 78,5 & 1511 & 2,09 \\
\hline $\mathrm{AuNw} 16$ & & 104 & 72,4 & 1384 & 1,52 \\
\hline $\mathrm{AuNw} 25$ & & 87 & 82,0 & 1753 & 5,26 \\
\hline $\mathrm{AuNw} 27$ & & 136 & 67,5 & 1275 & 1,26 \\
\hline $\mathrm{AuNw} 28$ & & 105 & 70,5 & 1303 & 1,00 \\
\hline $\mathrm{AuNw} 30$ & Entl. & 72 & 80,0 & 1852 & 7,43 \\
\hline AuNw31 & Entl. & 69 & 83,5 & 1822 & 7,58 \\
\hline $\mathrm{AuNw} 34$ & Entl. & 112 & 70,0 & 1595 & 0,85 \\
\hline $\mathrm{AuNw} 37$ & & 92 & 82 & 1651 & 1,18 \\
\hline $\mathrm{AuNw} 39$ & & 64 & 70,3 & 1525 & 15,43 \\
\hline Plate2 & & 130 & 68,2 & 1470 & 1,47 \\
\hline Dis8 & & 117 & 71,7 & 1537 & 1,25 \\
\hline Canti1 & $\begin{array}{l}\text { Cantilever statt Kraft- } \\
\text { sensor }\end{array}$ & 64 & 66,5 & 1762 & 2,15 \\
\hline AuNw17 & 'blind' & 114 & 77,0 & 1625 & 0,62 \\
\hline $\mathrm{AuNw} 18$ & 'blind' & 72 & 82,8 & 1661 & 0,77 \\
\hline $\mathrm{AuNw} 22$ & 'blind' & 87 & 74,1 & 1837 & 1,0 \\
\hline $\mathrm{AuNw} 23$ & 'blind' & 92 & 84,7 & 2164 & 3,9 \\
\hline Dis5 & Vorverformung & 100 & 85,5 & - & 0,23 \\
\hline Dis6 & Vorverformung & 100 & 83,4 & 1647 & 1,32 \\
\hline Dis7 & Vorverformung & 91 & 82,0 & 1650 & 3,03 \\
\hline $\mathrm{H} 2 \mathrm{O} 1$ & LowVacuum & 89 & 72,5 & 1298 & 1,72 \\
\hline $\mathrm{H} 2 \mathrm{O} 4$ & LowVacuum & 108 & 62,9 & 1306 & 0,91 \\
\hline$\overline{\mathrm{AuNw} 1}$ & ZZwillingsgrenze & 88 & 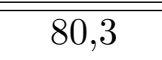 & 1785 & 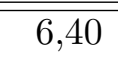 \\
\hline $\mathrm{AuNw} 5$ & Zwillingsgrenze & 141 & 77,5 & 1309 & 1,58 \\
\hline $\mathrm{AuNw} 26$ & Zwillingsgrenze & 92 & 81,0 & 1773 & 11,00 \\
\hline AuNw32 & Zwillingsgrenze + Entl. & 64 & 76,8 & 1618 & 6,05 \\
\hline $\mathrm{AuNw} 33$ & Zwillingsgrenze & 82 & 73,7 & 1669 & 2,45 \\
\hline $\mathrm{AuNw} 35$ & Zwillingsgrenze & 104 & 74,8 & 1611 & 1,83 \\
\hline $\mathrm{AuNw} 36$ & Zwillingsgrenze + Entl. & 131 & 76,2 & 1824 & 0,78 \\
\hline AuNw38 & Zwillingsgrenze & 110 & 62,3 & 1224 & 1,92 \\
\hline
\end{tabular}

Tabelle 4.4.: Zusammenfassung der Messergebnisse der Drähte aus dem REM 
theoretischen Wertes von 80,5GPa für Gold in <110>-Richtung liegt [58]. Abbildung 4.50 zeigt das E-Modul in Abhängigkeit vom Durchmesser der Drähte. Die verschiedenen Testvarianten bzw. Probentypen sind farblich kodiert. Ein Trend im Zusammenhang mit dem Durchmesser ist für das E-Modul nicht auszumachen. Ein Vergleich der verschiedenen Testvarianten zeigt ebenfalls keinen erkennbaren Trend.

\section{Fließspannung}

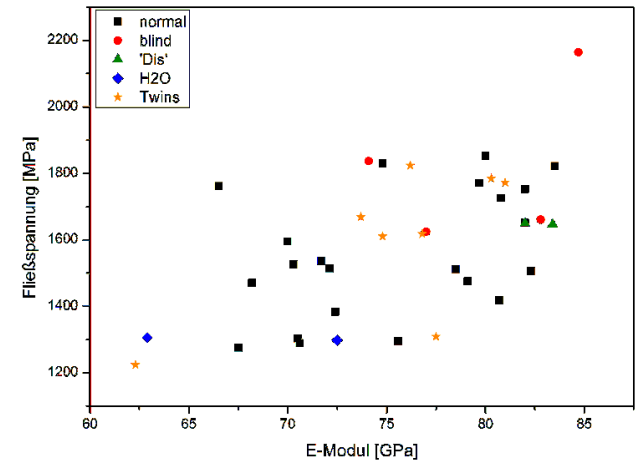

(a)

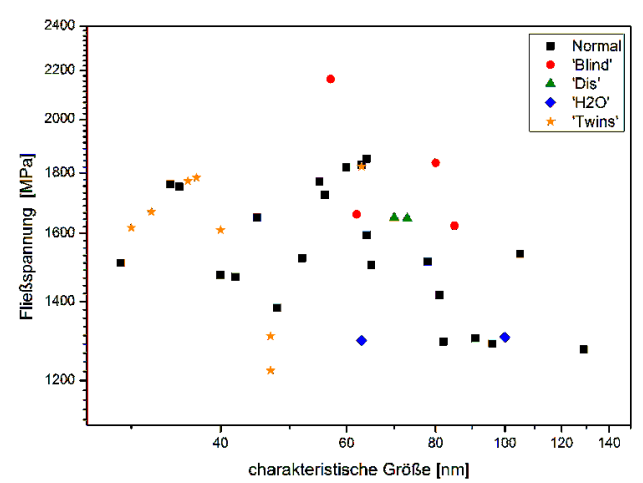

(b)

Abbildung 4.51.: (a) Fließspannung gegen das E-Modul; (b) Fließspannung gegen die charakteristische Größe

Die Fließspannung streut über einen großen Bereich zwischen 1200MPa und 2200Pa, wobei der Großteil der Drähte im Bereich bis 1900MPa liegt und nur ein Draht eine Fließspannung größer als 2000MPa aufweist. Abbildung 4.51 (a) zeigt die Fließspannung gegen das E-Modul. Der Draht mit der größten Fließspannung weist ebenfalls das größte E-Modul auf. Weiterhin weisen die beiden Drähte mit dem kleinsten E-Modul ebenfalls eine kleine Fließspannung auf. Für die restlichen Drähte ist jedoch kein Trend auszumachen, so dass man davon ausgehen kann, dass die beiden Größen unabhängig voneinander sind. Somit scheint ein systematischer Fehler in der Bestimmung der Querschnittfläche und der Kraft ausgeschlossen, da dies zu einem linearen Zusammenhang zwischen E-Modul und Fließspannung führen müsste.

In Abbildung 4.51 (b) ist die Fließspannung gegen die charakteristische Größe der Drähte in doppelt logarithmischer Auftragung zu sehen, wobei in Anlehnung an die Literatur die kürzere Dimension des Querschnitts als charakteristische Größe verwendet wurde [59][60]. Die verschiedenen Varianten sind wieder farblich kodiert. Es ist kein genereller Trend mit der Dimension der Drähte zu erkennen. Legt man beispielsweise 
eine Ausgleichsgerade durch alle Datenpunkte, so erhält man lediglich eine Steigung von $\sigma_{\text {flow }} \propto d^{-0,09}$. Es ist weiterhin kein Trend mit einer der Testbedingungen auszumachen. Hierbei fällt lediglich auf, dass alle Drähte die 'blind' getestet wurden, eine Fließspannung von mehr als 1600MPa aufweisen und damit alle im oberen Bereich der gemessenen Fließspannungen liegen.

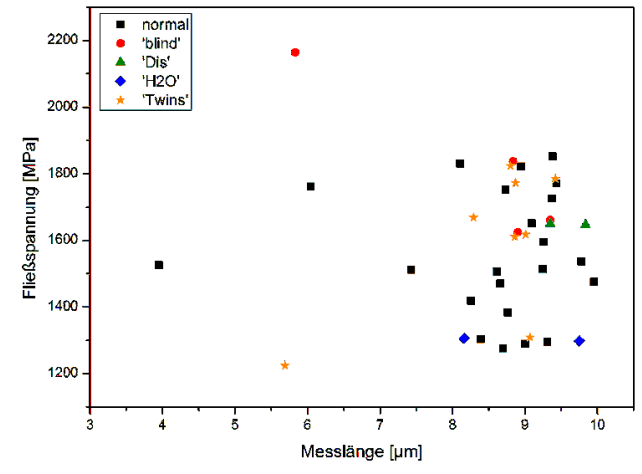

(a)

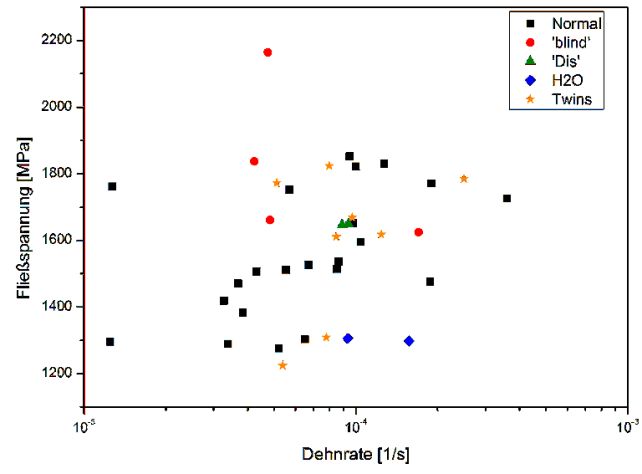

(b)

Abbildung 4.52.: Fließspannung gegen die Messlänge (a) und gegen die Dehnrate (b)

Die Messlänge der Drähte variiert zwischen $4 \mu m$ und 10 $\mu m$. Der Großteil der Drähte weist eine Messlänge zwischen $8 \mu m$ und $10 \mu m$, lediglich 4 Drähte liegen unterhalb dieses Bereichs. Abbildung 4.52 (a) zeigt die Fließspannung gegen die Messlänge. Ein eindeutiger Trend ist auch hier nicht auszumachen. Der Ausreißer mit der höchsten Fließspannung weist zwar auch eine kleine Messlänge auf. Die beiden anderen Drähte mit ebenfalls kleiner Messlänge bestätigen diesen Zusammenhang jedoch nicht. Die Dehnrate für die Zugversuche wurde im Verlauf der Versuchsreihe innerhalb einer Größenordnung von $1,2 \cdot 10^{-4} / \mathrm{s}$ bis $3,6 \cdot 10^{-4} / \mathrm{s}$ variiert. Abbildung 4.52 (b) zeigt die Fließspannung gegen die Dehnrate. Hierauf lässt sich kein Zusammenhang für die Fließspannung mit der Dehnrate erkennen.

\section{Plastische Dehnung}

Die plastische Dehnung der Drähte variiert über einen großen Bereich von 0,23\% bis 15, 4\%. Der Mittelwert aller Drähte liegt bei 2, 8\%. Im Verlauf der Versuchsreihe wurden drei verschiedene Methoden angewandt, den Aktuator zu steuern und den Draht damit zu verformen. Zunächst wurde der Aktuator per Hand mit einer minimalen Schrittgröße von $0,1 \mathrm{~V}$ gesteuert. Der Mittelwert der plastischen Dehnung dieser getesteten Drähte 
liegt bei 2, $0 \pm 1,7 \%$ und damit unter dem Durchschnitt aller getesteten Drähte. Danach wurde der Aktuator durch ein auf Matlab basierendem Programm mit einer minimalen Schrittgröße von 0,01V bewegt. Der Mittelwert der plastischen Dehnung liegt hier bei $1,7 \pm 0,9 \%$ und damit noch weiter unter dem Durchschnitt als der Mittelwert mittels Handsteuerung getesteten Drähte, obwohl hier die Schrittweite wesentlich kleiner war. Schließlich wurde dazu übergegangen, den Aktuator durch einen Funktionsgenerator zu steuern. Hier wurde keine Schrittgröße verwendet, sondern ein linearer Anstieg der Spannung vorgegeben. Der Mittelwert der plastischen Dehnung liegt hier bei 4, $6 \pm 4,5 \%$ und damit über dem Durchschnitt.

\begin{tabular}{c|c|c} 
per Hand & Computer & Funktionsgenerator \\
\hline $2,0 \pm 1,7 \%$ & $1,7 \pm 0,9 \%$ & $4,6 \pm 4,5 \%$
\end{tabular}

Tabelle 4.5.: Mittelwert der plastischen Dehnung für verschiedene Aktuatorsteuerungen

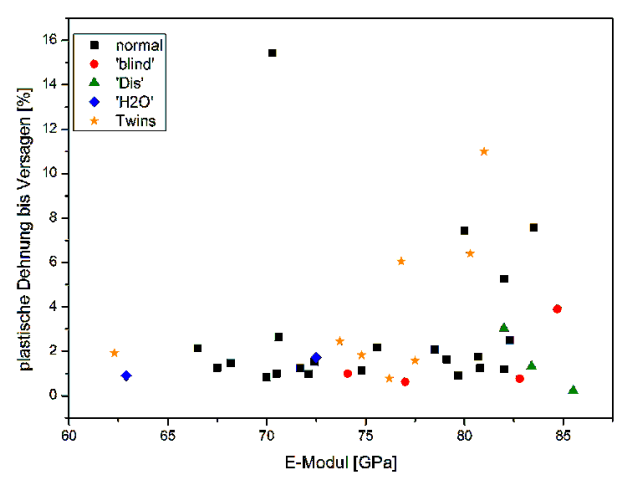

(a)

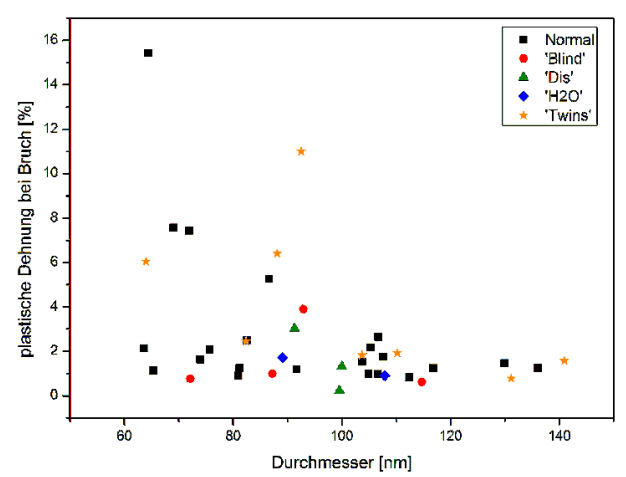

(b)

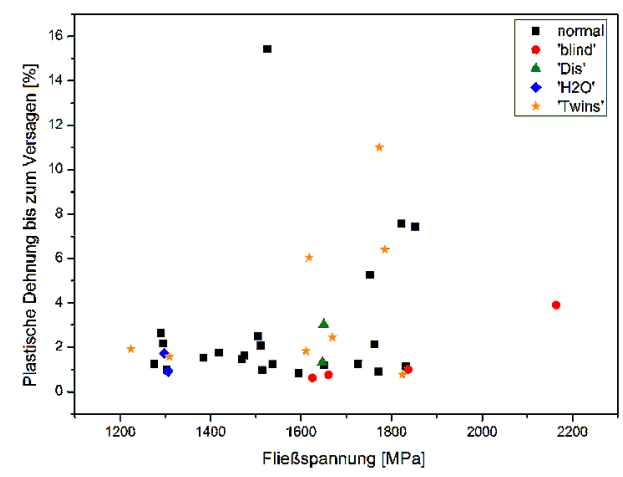

(c)

Abbildung 4.53.: Plastische Dehnung bis zum Versagen gegen das E-Modul (a), gegen den Durchmesser (b) und gegen die Fließspannung (c) 
Abbildung 4.53 (a) zeigt die plastische Dehnung bis zum Versagen gegen das EModul. Ein klarer Trend ist erneut nicht auszumachen. Allerdings weisen alle Drähte, die eine plastische Dehnung von mehr als 3\% erreicht haben, auch ein großes E-Modul von mehr als 76GPa auf. Die einzige Ausnahme und Ausreißer stellt AuNw39 dar, der bei einem E-Modul von 70,3GPa die größte plastische Dehnung sämtlicher Drähte in Höhe von $15,4 \%$ aufweist.

Abbildung 4.53 (b) zeigt die plastische Dehnung bis zum Versagen gegen den Durchmesser der Drähte. Es ist ebenfalls kein klarer Trend zu erkennen, so dass die plastische Dehnung offenbar größenunabhängig ist. Allerdings existieren die Ausreißer nur bei kleinen Drähten, d.h. sämtliche Drähte, die eine plastische Dehnung von mehr als $3 \%$ aufweisen, haben einen Durchmesser, der kleiner als 95nm ist.

Abbildung 4.53 (c) zeigt die plastische Dehnung bis zum Versagen gegen die Fließspannung mit 1\% Offset-Dehnung. Es ist wiederherum kein klarer Trend auszumachen. Die Ausreißer mit einer hohen plastischen Dehnung von mehr als 3\% weisen jedoch alle gleichzeitig eine Fließspannung auf, die größer als 1600MPa ist.

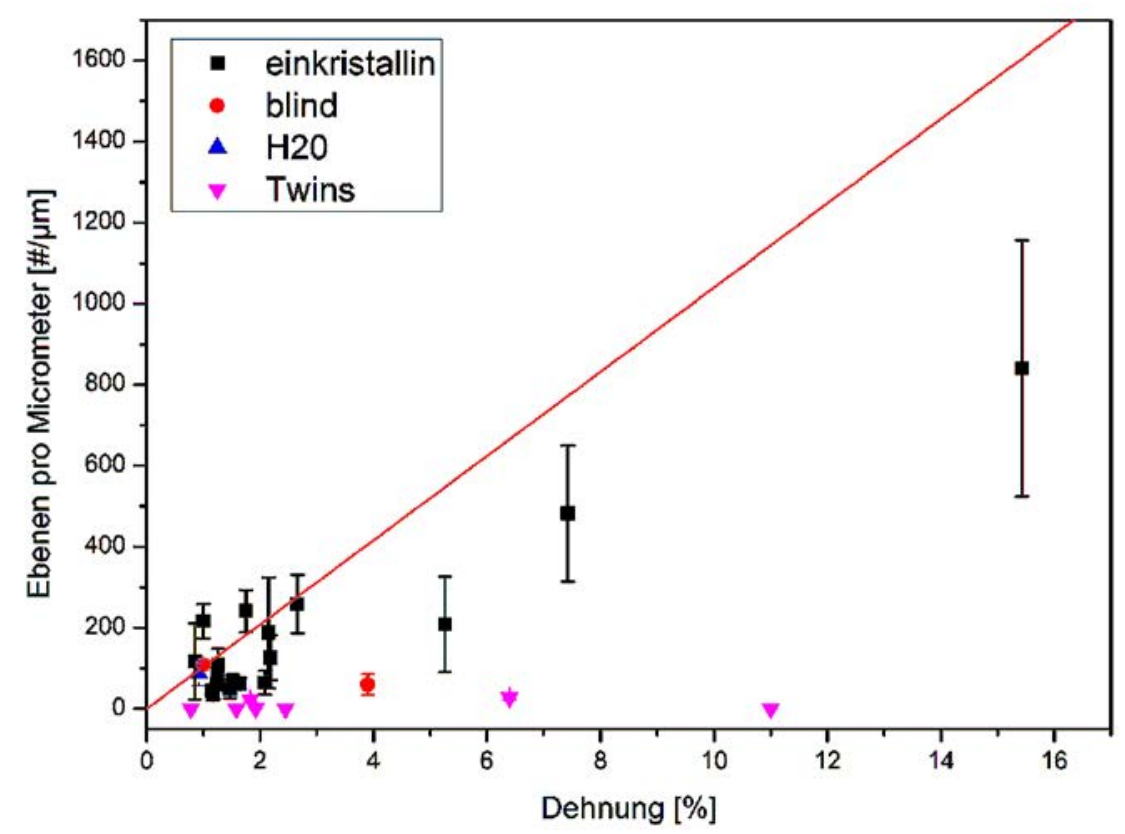

Abbildung 4.54.: Anzahl an Ebenen in den gespeicherten Defekten pro Mikrometer gegen die Dehnung; die rote Linie stellt die benötigte Anzahl an Ebenen pro Dehnung dar

Abbildung 4.54 zeigt die Anzahl an Ebenen in den gespeicherten Defekten pro Mikrometer gegen die Dehnung. Die verschiedenen Test- bzw. Probentypen sind 
wiederherum farblich markiert. Nimmt man an, dass das Durchlaufen von leadingPartialversetzungen die Ursache für planaren Defekte ist und jede Partialversetzung genau zur Verschiebung einer Ebene führt, lässt sich aus dem Burgersvektor und der Geometrie der Zugversuche die benötigte Anzahl an Ebenen berechnen, um eine gewisse Dehnung zu erzeugen. Dies ist durch die rote Linie dargestellt. Aus Abbildung 4.54 ist ersichtlich, dass im allgemeinen Fall die gespeicherten Defekte pro Dehnung unterhalb der Linie liegen. Ausnahmen bilden einige Drähte, die nur eine geringe plastische Dehnung erfahren haben, aber gleichzeitig einen großen Zwilling an der Bruchkante aufweisen. Hierbei ist davon auszugehen, dass dieser Zwilling im Zuge des Reißens entstanden ist (siehe auch Abschnitt 4.2.4) und somit die dadurch entstandene Dehnung nicht mehr in die DIC-Analyse einfließt. Aus Abbildung 4.54 wird also deutlich, dass leading- und twinning-Partialversetzungen alleine nicht ausreichen, um die plastische Dehnung in den Drähten zu erklären. 


\section{Zusammenfassung Spannungs-Dehnungs-Kurven}

- Das E-Modul unterliegt einer großen Streuung von 62GPa bis 86GPa. Dabei ist keine Abhängigkeit von dem Durchmesser ersichtlich. Die Fließspannungen zeigen ebenfalls eine große Streuung zwischen 1,2GPa und 1,2GPa. Ein Vergleich zwischen Fließspannung und E-Modul zeigt keinen klaren Zusammenhang, so dass systematische Fehler in der Messung der Querschnittsfläche oder Kraft auszuschließen sind.

- Die Fließspannungen zeigen ebenfalls keinen klaren Zusammenhang mit der Dimension der Drähte in dem hier untersuchten Bereich zwischen 20nm und 140nm. Weiterhin ist kein eindeutiger Trend gegen die Dehnrate für den Bereich zwischen $1,2 \cdot 10^{-5} / \mathrm{s}$ und $3,6 \cdot 10^{-4} / \mathrm{s}$ oder für die Drahtlänge im Bereich zwischen $4 \mu \mathrm{m}$ und $10 \mu \mathrm{m}$ zu erkennen.

- Die plastische Dehnung bis zum Bruch streut über einen großen Bereich von 0,6\% bis $15,4 \%$. Dabei scheint die plastische Dehnung größenunabhängig zu sein, wobei Ausreißer nur bei kleineren Drähten $(<95 \mathrm{~nm})$ auftauchen. Dagegen scheint die plastische Dehnung empfindlich auf die Steuerung des Aktuators zu reagieren, wobei Versuche mit dem Funktionsgenerator im Mittel höhere plastische Dehnungen aufweisen.

- Einkristalline Drähte zeigen einen graduellen Übergang zur plastischen Verformung. Während der plastischen Verformung kann man nur sehr geringe Spannungsabfälle beobachten. Im Ganzen bleibt die Fließspannung jedoch sehr konstant. Bis auf einen kleinen Abfall zu Beginn der plastischen Verformung zeigen verzwillingte Drähte ein sehr ähnliches Spannungs-Dehnungs-Verhalten. So ist insbesondere kein auffallender Unterschied in der Fließspannung im Vergleich zu einkristallinen Drähten feststellbar. 


\section{Diskussion}

\subsection{Elastisches Verhalten}

Bis zu einer Dehnung von circa 1\% zeigen sämtliche Drähte ein linear elastisches Verhalten, das komplett reversibel ist, wie durch die Entlastungsversuche gezeigt wurde. Ab circa 1\% Dehnung ist eine nichtlineare Elastizität bei den Drähten zu beobachten. Reversibles Verhalten wurde dabei durch die Entlastungsexperimente bestätigt, wobei die Entlade-Kurve einen nichtlinearen Verlauf zurück auf die Ausgangsspannung aufweist. Dieses Verhalten von nicht-linearer Elastizität bei Dehnungen größer als 1\% wird durch MD-Simulationen [40][61] und durch experimentelle Zugversuche in Nanodrähten bestätigt [62][41], was auf eine Elastizität höherer Ordnung zurückgeführt wird.

Das ermittelte E-Modul im Bereich bis zu einer Dehnung von 1\% weist eine relativ große Streuung zwischen 62GPa und 86GPa auf, bei einem Mittelwert von 76GPa. Dieser Wert liegt nahe am theoretischen Wert für Bulk-Gold in $\langle 110\rangle$-Richtung (80, 9GPa [40] bzw. 81, 5GPa [58]). Ein Einfluss der Zwillingsgrenze auf das E-Modul konnte nicht festgestellt werden. Dies wird durch atomistische Berechnungen an verzwillingten Silberdrähten bestätigt, bei denen kein signifikanter Unterschied im E-Modul durch die Präsenz von Zwillingsgrenzen festgestellt werden konnte [63]. Ergebnisse anderer Arbeiten in der Literatur an $\langle 110\rangle$-Golddrähten zeigen ähnliche Werte von 72GPa [20], 68GPa [28] oder eine noch größere Streuung von 20GPa bis 130GPa [60].

Als Grund für die hohe Streuung der Daten wurde in [60] als wahrscheinlichster Fehler die Bestimmung der Querschnittfläche ausgemacht. In den Versuchen dieser Arbeit wurde jedoch circa die Hälfte der Querschnitte im TEM vermessen, was bis dato die höchste Genauigkeit erlaubt. Ein Unterschied in der Streuung des E-Moduls zu Drähten, in denen der Querschnitt mit dem FIB bestimmt wurde, lässt sich jedoch nicht erkennen. Weiterhin zeigt sich keine Korrelation zwischen E-Modul und Fließspannung, 
was eine fehlerhafte Bestimmung der Fläche ausschließt.

Murphy et al. [64] berichten von einer fehlerhaften Bestimmung des E-Moduls von bis zu 39\% durch Rutschen und Nachgiebigkeit der organo-metallischen Abscheidungen, die als Klemmen verwendet werden. Ein solcher Effekt konnte für diese Arbeit durch ein Marker-Experiment ausgeschlossen werden, indem die Dehnung zwischen den beiden Klemmen und Pt-Markern auf dem Draht als identisch bestimmt werden konnte (siehe Abschnitt 3.3.2). Zu erwähnen ist allerdings, dass ein solches Marker-Experiment im Zuge dieser Arbeit nur einmal durchgeführt wurde. Frühere Arbeiten [12][14] bestätigen jedoch auch dieses Bild und zeigen darüber hinaus sogar leicht erhöhte Dehnungen zwischen den Markern. Dies stellt ein entgegengesetztes Verhalten dar, als man es aufgrund der Nachgiebigkeit der Platinklemmen erwarten würde. Die Platinklemmen scheiden somit als Fehlerquelle aus.

Eine weitere mögliche Fehlerquelle stellt die Abweichung von der uniaxialen Zugrichtung dar. Die Inplane-Abweichung zur Zugachse konnte im Transferprozess für alle Drähte unter $2^{\circ}$ gehalten werden, so dass hieraus keine signifikanten Fehler zu erwarten sind [65]. Eine genaue Aussage über die Ausrichtung zur z-Komponente aus der Bildebene des Elektronenstrahls heraus lässt sich nur schwer ableiten, da diese Ausrichtung nur im Vorfeld eines Versuchs durch den Manipulator eingestellt werden kann (siehe Abschnitt 3.3). Ein Kippen des REM-Tisches nach dem Versuch lässt Einblicke in diese out-of-plane-Ausrichtung zu. Dabei konnten ebenfalls keine Anzeichen für eine nennenswerte Abweichung von der Zugachse festgestellt werden. Allerdings lassen Verschiebungen der Drahtenden zueinander durch Vibrationen beim Kippen und eine etwaige Verbiegung des Drahtes (z.B. durch eine erhöhte Kontamination auf der Oberseite des Drahtes durch den Elektronenstrahl) nur eine grobe Abschätzung zu. Ein weiterer Indikator für eine gute Ausrichtung der Drähte ist, dass sie gewöhnlich weit entfernt von den Klebestellen reißen. Die Abweichung von der uniaxialen Zugrichtung lässt sich somit auch als signifikante Fehlerquelle ausschließen.

Als physikalischer Grund für ein abweichendes E-Modul kommt eine frühe Plastizität in Frage. In den TEM-Experimenten ließen sich jedoch keine Hinweise auf anfängliche Plastizität in den Drähten beobachten (> 1\%-Dehnung in dem Marker-Experiment ohne ein Auftauchen von Defekten). Weiterhin konnten bei Drähten aus den REMVersuchen keine ausreichende Zahl an Defekten nachträglich festgestellt werden, die für eine solche Abnahme des E-Moduls erforderlich gewesen wären. 
Als ein weiterer physikalischer Grund käme ein Größeneffekt des E-Moduls in Betracht. In Nanostrukturen findet man ein ansteigendes Oberfläche zu VolumenVerhältnis vor, so dass üblicherweise zwei Effekte für eine Größenabhängigkeit des E-Moduls zu beachten sind: erstens die Oberflächenelastizität, wobei die Oberflächenatome ein anderes Modul aufweisen als die Bulk-Moleküle, und zweitens die Oberflächenspannungen, die zu Kompression- oder Zugspannungen der Kern-Atome führen. Einfache Überlegungen [12] zeigen, dass beide Effekte erst ab einer Probengröße unterhalb von 5nm einen messbaren Effekt zeigen und damit für den in dieser Arbeit getesteten Bereich keinen Einfluss haben sollten. Dies wird gestützt durch atomistische Simulationen an Nanodrähten bzw. Nanostrukturen, die ebenfalls erst einen Einfluss unterhalb von 10nm erkennen lassen [66][67][68].

Demgegenüber liefern experimentelle Studien an Nanodrähten der verwendeten Größenordnung widersprüchliche Ergebnisse. Auf der einen Seite zeigen Studien an nominell identischen Golddrähten dieses Größenbereichs keinen Größeneffekt [60]. Andere Studien von Zugversuchen an fcc-Metall-Nanodrähten in $\langle 110\rangle$-Richtung zeigen jedoch bei abnehmendem Durchmesser von 100nm auf 30nm einen Anstieg des E-Moduls um 100\% [62][69]. Ein Größeneffekt für das E-Modul konnte jedoch in den Versuchen für diese Arbeit nicht festgestellt werden, so dass diese Ergebnisse besser mit den Simulationen und den Arbeiten in [60], [20] und [28] übereinstimmen. Insbesondere lag das E-Modul in allen diesen Studien eher niedriger als der Bulk-Wert und zeigte keinen Anstieg wie in [62] und [69]. Die Abweichungen erklären sich möglicherweise durch die Verwendung unterschiedlicher Materialien. So wurden in [62] Palladium-Nanodrähte untersucht und in [69] Silber-Nanodrähte.

Zusammenfassend lässt sich festhalten, dass durch die sorgfältige Herangehensweise in den Versuchen dieser Arbeit die Streuung für das E-Modul im Vergleich zu anderen Arbeiten, bei denen experimentelle Ungenauigkeiten nicht auszuschließen sind, deutlich reduziert werden konnte. Die verbleibende restliche Streuung sowie das steigende E-Modul mit zunehmender Dehnung in den Entlastungsversuchen machen jedoch deutlich, dass für das elastische Verhalten von Nanodrähten weiterhin Fragen offen bleiben, für die in dieser Arbeit keine Antworten gefunden werden konnten. 


\subsection{Nukleationskontrollierte Verformung}

Eine Schlüsselfrage in der Verformung in nanoskaligen Proben ist, in welcher Weise sich eine nukleationsbasierte Verformung im Vergleich zur herkömmlichen Verformung in Bulk-Kristallen unterscheidet. In sämtlichen Zugversuchen für diese Arbeit wurde das Auftreten und die Speicherung von Defekten beobachtet, die sich durch Präsenz von Partialversetzungen interpretieren lassen. Da die Drähte vor den einzelnen Versuchen größtenteils versetzungsfrei waren, kann dies ausschließlich durch Nukleation von Partialversetzungen verursacht worden sein. Damit unterscheidet sich eine nukleationskontrollierte Verformung deutlich von einer herkömmlichen Verformung in Bulk-Gold, bei der die Verformung in der Regel durch volle Versetzungen bestimmt wird. Damit zeigen die Ergebnisse eine gute Übereinstimmung sowohl zu vorangegangen Studien am gleichen Probenset [11][56] als auch weiteren Zug-Experimenten von $\langle 110\rangle$-GoldNanodrähten [70][71][20][72][21][22][28].

Wie die Ergebnisse weiter gezeigt haben, ist dieses nukleationsbasierte Verhalten unabhängig von der Probengröße in dem hier untersuchten Bereich von $60 \mathrm{~nm}$ bis $140 \mathrm{~nm}$. Insbesondere konnte ein größenabhängiger Wechsel hin zu einer Verformung über volle Versetzungen, wie er durch das Modell von Chen et al. [46] angewandt auf $\langle 110\rangle$-Golddrähte über einem Durchmesser von $\approx 40 \mathrm{~nm}$ vorhergesagt wurde [21][28], nicht verifiziert werden. Dies mag an dem recht simplen Ansatz in diesem Modell liegen, dass lediglich die nötigen Spannungen für die Aktivierung einer Versetzungsquelle (im Allgemeinen Frank-Read-Quelle) betrachtet. Damit stimmen die Ergebnisse dieser Arbeit mit weiteren Arbeiten in der Literatur überein, die ebenfalls in Drähten bis zu einem Durchmesser von 150nm keinen Wechsel zu einer Verformung durch volle Versetzungen beobachten konnten [22][28]. Weiterhin haben Berechnungen aus atomistischen Simulationen gezeigt, dass das Aktivierungsvolumen für die Keimbildung bei der Versetzungsnukleation zwischen $10 b^{3}$ und $40 b^{3}$ für den in dieser Arbeit gemessenen Spannungsbereich der plastischen Verformung im Bereich zwischen 1GPa und 2GPa liegt [27]. Ebenfalls liegt der Radius für die Nukleation eines Halbrings in der klassischen Nukleationstheorie im Bereich zwischen 1nm und 3nm [11]. Sowohl das Aktivierungsvolumen als auch der Keimradius liegen also mehr als eine Größenordnung unter den Dimensionen der Drähte, so dass man auch hieraus nur einen schwachen Größeneffekt erwarten kann.

Wie durch die klassische Nukleationstheorie beschrieben wird, ist für die Nuklea- 
tionsspannung und damit für die Fließgrenze in den Drähten nur ein vernachlässigbarer Größeneffekt zu erwarten (siehe Gleichung 2.9). Die Werte für die Fließspannung in dieser Arbeit liegen im Bereich zwischen 1200MPa und 2200MPa und bestätigen damit den generellen Trend, dass kleine Proben größere Fließspannungen aufweisen. Obwohl die Streuung recht groß ist, sind diese Werte im Vergleich mit anderen Probensystemen, die nicht einer nukleationsbasierten Verformung unterliegen, recht konstant. Abbildung 5.1 zeigt einen Überblick der gemessenen Werte für die Fließspannung in dieser Arbeiten mit einigen in der Literatur vorhandenen Daten zu Gold.

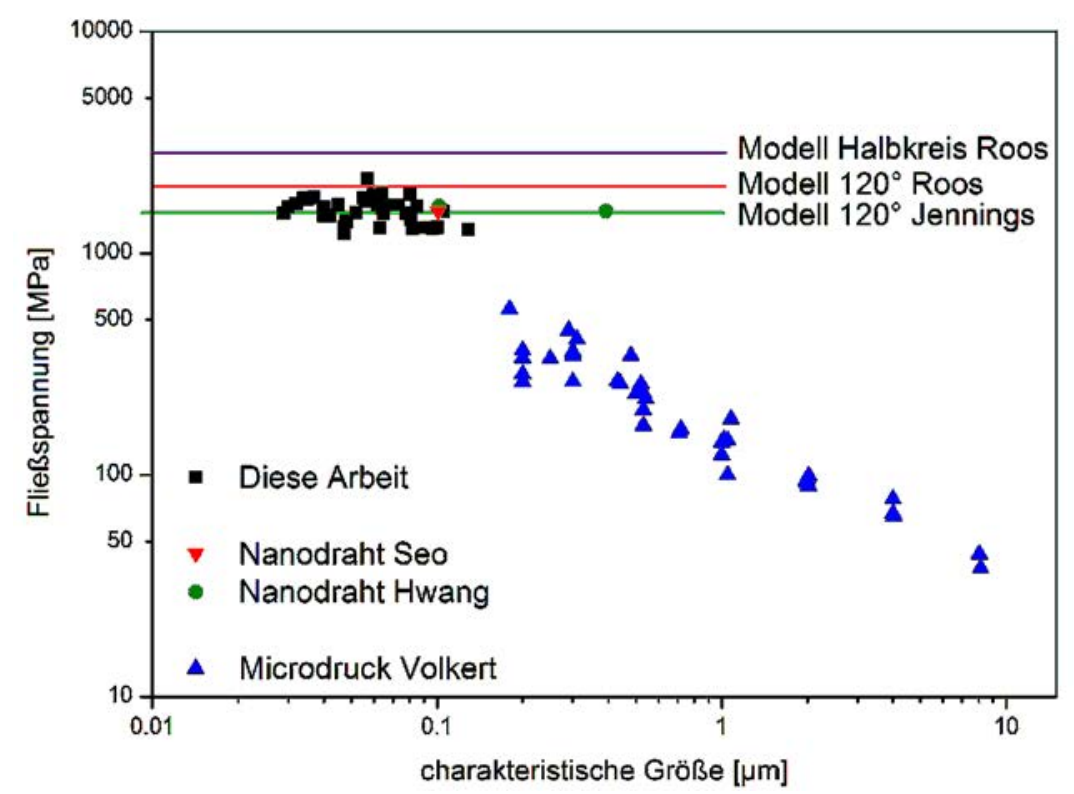

Abbildung 5.1.: Vergleich der Fließspannungen: diese Arbeit (schwarze Vierrecke); Nanodraht von Seo et al. [20] (rotes Dreieck); Nanodrähte von Hwang et al. [28](grüne Punkte); Säulen von Volkert und Lilleodden [73] (blaue Dreiecke). Die Linien zeigen die Nukleationsspannungen nach dem Modell von Roos [11] für einen Halbkreis (violette Linie), für eine $120^{\circ}$ Ecke (rote Linie) und nach dem Modell von Jennings et al. für eine $120^{\circ}$-Ecke (grüne Linie).

Die blauen Dreiecke zeigen die Fließspannungen von Druckversuchen an Goldsäulen von Volkert und Lilleodden [73]. Diese Fließspannungen zeigen einen starken Größeneffekt mit abnehmender Probengröße von $4 \mu \mathrm{m}$ auf $180 \mathrm{~nm}$ mit einer Abhängigkeit von $\sigma_{f} \propto d^{-0,6}$. Weitere Untersuchungen an Gold-Säulen [74][75][76] und auch anderen fcc-Metallsäulen [77][78] zeigen einen ähnlichen Größeneffekt.

Zusätzliche Daten für Nanodrähte liefern die Arbeiten von Seo et al. [20] sowie Hwang et al. [28]. Seo et al. [20] berichten von einer Fließspannung von 1540MPa 
für einen Nanodraht mit der kurzen Facttenlänge von 101nm (rotes Dreieck in Abbildung 5.1). Hwang et al. [28] berichten von einer konstanten Fließspannung in ihren Golddrähten, wobei sie jedoch lediglich die expliziten Werte für zwei Drähte angeben: 1,63GPa für einen 102nm-Draht und 1,55GPa für einen 392nm-Draht (grüne Punkte in Abbildung 5.1).

Die gemessenen Fließgrenzen dieser Arbeit sind in Abbildung 5.1 als schwarze Vierecke dargestellt. Im Vergleich mit den Werten aus den Druckversuchen erweist sich die Fließspannung im Rahmen dieser Arbeit $\left(\sigma_{f} \propto d^{-0,09}\right)$ und auch zusammen mit den Ergebnissen der weiteren Nanodrähte als sehr konstant. Wie in Abschnitt 2.5 beschrieben, ist für die nukleationsbasierte Verformung nur ein unbedeutender Größeneffekt für die Festigkeit zu erwarten. So zeigt das Fehlen eines Größeneffekts in der Fließspannung der Drähte eine gute Übereinstimmung mit der klassischen Nukleationstheorie und einen deutlichen Unterschied zu dem Größeneffekt in den Druckversuchen der Säulen, in denen man von einer Verformung durch die Aktivierung von vorhandenen Versetzungen sowie deren Reaktionen mit anderen Versetzungen und mikrostrukturellen Merkmalen ausgeht.

Vergleicht man allerdings die gemessen Werte für die Fließgrenze (zwischen 1,2GPa und 2,2GPa) mit dem Wert von 2,85GPa aus der klassischen Nukleationstheorie nach dem Modell von Roos [11] (violette Linie in Abbildung 5.1), zeigt sich eine merkliche Diskrepanz. In diesem Modell wurde von der Keimbildung eines Halbrings an der Oberfläche ausgegangen und zusätzlich die Bildkräfte nach Beltz und Freund [37] sowie die Reduktion einer Oberflächenstufe nach Hirel et al. [34] berücksichtigt.

Eine mögliche Anpassung für das ursprüngliche Modell von Roos ist die Betrachtung der Keimbildung an einer Ecke, wie es auch in Simulationen beobachtet wurde [21][27]. Dadurch verändert sich zum einen die Länge des Linienelements, zum anderen müssen die Bildkräfte neu angepasst werden [35]. Für eine $120^{\circ}$-Ecke ergibt sich dann eine Nukleationsspannung für die leading-Partialversetzung von 2GPa. Dies deckt sich mit dem Ausreißer in den gemessenen Fließspannungen. Da der Großteil der Drähte jedoch eine Fließspannung kleiner als 2GPa aufweist, stellt auch diese Anpassung noch keine gute Übereinstimmung zwischen Modell und Experiment dar. Weiterhin ist fraglich, ob in den verwendeten Drähten die Annahme einer $120^{\circ}$-Ecke zutreffend ist. Im Gegensatz zu den Simulationen weisen die Drahtquerschnitte der für diese Arbeit verwendeten Drähte Abrundungen im Bereich zwischen circa 5nm und 10nm auf, die 
größer sind als der Keimradius $(\approx 2 n m)$. Es ist also anzunehmen, dass die tatsächliche Konfiguration in der Mitte zwischen der Keimbildung von einem Halbring und an einer $120^{\circ}$-Ecke liegt und die Reduktion der Nukleationsspannung durch diese geometrische Anpassung dementsprechend kleiner ausfällt.

Jennings et al. [35] haben neben der Betrachtung einer 120-Ecke mit den entsprechenden Bildkräften zusätzlich die Werte für den Burgersvektor, die Stapelfehlerenergie sowie den inneren Kernradius der Versetzung angepasst. Dagegen haben sie nicht die Reduktion einer Oberflächenstufe betrachtet, sondern die Ausbildung einer Oberflächestufe. Damit erhalten sie für die Nukleationsspannung der leading-Partialversetzung einen Wert von 1,52GPa (grüne Linie in Abbildung 5.1), die eine sehr gute Übereinstimmung mit den experimentell gemessen Fließspannungen zeigt. In den verwendeten Drähten konnten jedoch Oberflächenstufen eindeutig nachgewiesen werden. Würde man in dem Modell von Jennings et al. statt der Ausbildung von der Reduktion einer Oberflächenstufe ausgehen, würde dies zu einer Unterschätzung der Nukleationsspannung führen. Andersherum würde der Einfluss eines abgerundeten Querschnitts zu einer Erhöhung der Nukleationsspannung führen müssen. Beide Effekte, Reduktion einer Oberflächenstufe und Abrundung der Querschnitte, würden sich also abschwächen bzw. kompensieren.

Es lässt sich also festhalten, dass die Nukleationsspannung für eine leadingPartialversetzung aus der klassischen Nukleationstheorie durchaus in Einklang zu bringen ist mit den experimentell bestimmten Fließspannungen. Für das ursprüngliche Modell von Roos sind dafür allerdings einige Variationen erforderlich.

Nach der klassischen Nukleationstheorie (Gleichung 2.10) ist die Nukleationsspannung nur sehr schwach von der Dehnrate abhängig $\left(\sigma_{y} \propto \ln [1 / \dot{\epsilon}]\right)$. Dies konnte in dieser Arbeit bestätigt werden. So zeigen die Ergebnisse in Abbildung 4.52 keine Abhängigkeit der Fließspannung für Dehnraten im Bereich zwischen $1 \cdot 10^{-5} / \mathrm{s}$ und $5 \cdot 10^{-4} / \mathrm{s}$. Dies deckt sich ebenfalls mit Zugversuchen an Palladium-fcc-Nanodrähten in $\langle 110\rangle$-Richtung, die ebenfalls keine Abhängigkeit der Fließspannung in einem ähnlichen Dehnraten-Bereich ergaben [41].

Aus Abbildung 4.52 kann man ebenfalls keine Abhängigkeit der Fließspannung mit der Länge der Drähte im Bereich von $4 \mu m$ bis $10 \mu m$ ausmachen. Geht man von einer homogenen Verteilung äquivalenter Nukleationsplätze entlang des Drahtes aus, so entspräche der abgedeckte Bereich der Drahtlänge etwas mehr als einer Verdoppelung 
der Nukleationsplätze. Folgt man Gleichung 2.10, so ist kein deutlicher Effekt durch eine Verdopplung der Nukleationsplätze $N$ durch die schwache Abhängigkeit der Nukleationsspannung $\left(\sigma_{f} \propto \ln (N)\right)$ zu erwarten. So zeigen auch Pd-Nanodrähte nur eine marginale Abhängigkeit der Spannung von der Länge der Drähte im Bereich zwischen $1 \mu m$ und $8 \mu m[41]$.

\section{Einfluss der anfänglichen Versetzungsdichte}

Eine weitere Schlüsselfrage hinsichtlich der Plastizität auf der Nanoebene ist unter welchen Bedingungen eine nukleationskontrollierte Verformung überwiegt gegenüber einer herkömmlichen Verformung durch volle Versetzungen. Wie in der Literatur beschrieben, kann die anfängliche Versetzungsdichte einen maßgeblich Einfluss sowohl auf die Festigkeit als auch auf das Verformungsverhalten haben [6][79][80][81][82][83][7]. Ein häufig untersuchtes Probensystem sind hierbei Fibern einer Molybdän-Verbindung mit einer variablen Versetzungsdichte [79][80][81][82]. Versetzungsfreie Fibern zeigen eine sehr hohe Festigkeit nahe der theoretischen Festigkeit und ein katastrophales Versagen nach Erreichen der Fließgrenze, was typisch ist für nanoskalige kubisch raumzentrierte Proben und auf einen Lawineneffekt nach der Nukleation der ersten Versetzung zurückgeführt wird [84]. Fibern mit einer hohen Versetzungsdichte zeigen dagegen eine Festigkeit nahe dem bulk-Wert, gefolgt von einer ausgeprägten plastischen Verformung. Fibern mit einer mittleren Versetzungsdichte weisen eine markante Streuung der Festigkeit von hohen Werten wie in den versetzungsfreien Proben bis hinunter zu kleinen Werten wie in den versetzungsreichen Proben auf.

Mompiou et al. [83] haben herausgestellt, dass es scheinbar nicht nur auf die Versetzungsdichte ankommt, sondern auch auf die Orientierung der Versetzung, so dass diese als aktive Quelle für weitere Versetzungen dienen kann. So haben sie beobachtet, dass bereits eine einzelne Versetzung als Quelle für viele weitere Versetzungen dienen konnte und die Verformung somit nicht auf die Nukleation von neuen Versetzungen angewiesen ist. Eine hohe Versetzungsdichte führt also nur zu einer erhöhten Wahrscheinlichkeit, eine aktive Quelle in der Probe zu finden, wohingegen eine geringe Versetzungsdichte somit nur eine kleine Wahrscheinlichkeit für eine aktive Quelle darstellt.

Im Rahmen dieser Arbeit ließ sich jedoch kein besonderer Einfluss von anfänglichen Versetzungen auf das Verformungsverhalten beobachten. Zwar konnte eine Be- 
wegung der Versetzungen festgestellt werden, so dass es sich tatsächlich um mobile Versetzungen gehandelt haben muss. Es konnte aber nicht beobachtet werden, dass die Versetzungen als Quelle für weitere Versetzungen gedient hätten. Stattdessen haben die eingebrachten Versetzungen die Probe verlassen, ohne dass diese vorher als Quelle für neue Versetzungen gedient hätten oder es vorher zu einer Wechselwirkung mit anderen Versetzungen gekommen wäre, was ebenfalls schon in der Literatur beschrieben wurde [5][82]. Die anschließende Verformung erfolgt dann wieder kontrolliert durch die Nukleation von neuen Versetzungen. Ebenfalls konnte kein Einfluss auf die Festigkeit der Probe festgestellt werden. Auf der einen Seite hat demnach die Dichte der eingebrachten Versetzungen nicht ausgereicht, um eine selbsterhaltende Population von Versetzungen zu erreichen bzw. zu erzeugen. Auf der anderen Seite waren die eingebrachten Versetzungen anscheinend nicht von der Art, die als Quelle für weitere Versetzungen dienen konnten.

Festzuhalten bleibt hierzu, dass es durch die Beanspruchung vor dem Zugversuch zwar möglich war, Versetzungen in die Nanodrähte einzubringen und somit den Einfluss auf das Verformungsverhalten zu untersuchen. Allerdings war dies mit einem enormen experimentellen Aufwand verbunden, mit dem ebenfalls eine Reihe von Nachteilen verbunden sind (Dellen im Draht, Ausrichtung der Drähte). Weiterhin ließ sich nicht ohne weiteres die eingebrachte Versetzungsdichte kontrolliert steuern, um so gezielt den Einfluss einer variablen Versetzungsdichte zu untersuchen. Weiterhin wäre es für diese speziellen Versuche eventuell erforderlich, einen quantitativen TEM-Aufbau zu verwenden, um so feststellen zu können, bei welchen Spannungen die gespeicherten Versetzungen aktiviert werden. Somit sollten die für diese Arbeit durchgeführten Versuche mit eingebrachten Versetzungen als Ausgangspunkt für nachfolgende Untersuchungen angesehen werden.

\subsubsection{Nukleation von leading-Partialversetzungen}

Wie die Ergebnisse dieser Arbeit gezeigt haben, ist das erste Nukleationsevent die Bildung einer leading-Partialversetzung, die in ihrer Spur einen Stapelfehler hinterlässt. Dies entspricht auch dem erwarteten Verhalten für Gold in $\langle 110\rangle$-Richtung, da die Nukleation einer leading-Partialversetzung durch den hohen Schmidfaktor $(\mathrm{m}=0,47)$ präferiert ist [19]. Aber auch während der gesamten weiteren Verformung kann das Auftauchen von separierten planaren Defekten und damit die Keimbildung von neuen 
leading-Partialversetzung beobachtet werden. Dies ist nach den Modellen von Weinberger et al. [19] und Roos [11] nicht das erwartete Verhalten und wird auch in MDSimulationen im allgemeinen Fall nicht beobachtet [18][21][28]. Es stellt sich also die Frage, was die Nukleation der leading-Partialversetzung verursacht bzw. wie die Keimbildung vonstatten geht.

Wie bei allen Formen von Keimbildung ist davon auszugehen, dass die Bildung eines Versetzungskeims an einer heterogenen Stelle wesentlich einfacher ist als eine homogene Keimbildung in Bulkmaterial. Bei Nanodrähten bieten sich die Oberflächen für eine solche heterogene Keimbildung an. Hier hat man es zum einen mit Spannungskonzentrationen aufgrund von Bildkräften zu tun und zum anderen verkleinert sich das Linienelement im Vergleich zu einer homogenen Nukleation im Kern des Drahtes [35]. Insbesondere stellen Oberflächendefekte wie zum Beispiel Oberflächenstufen einen besonders geeigneten Ort für die Keimbildung einer Versetzung dar [40][85][33][34][86]. Oberflächenstufen dienen zum einen als zusätzliche Spannungskonzentrationen, zum anderen kann durch die Nukleation einer Versetzung eine Oberflächenstufe verkleinert und dadurch die Energiebarriere für die Nukleation verringert werden. Roos [11] hat in seinem Modell den Einfluss durch den Abbau bzw. das Erzeugen einer Oberflächenstufe berücksichtigt und konnte auf diese Weise das Auftreten von separierten planaren Defekten durch eine Verteilung von Oberflächenstufen entlang der Drähte erklären. Erichsen [56] hat in seiner Arbeit die Präsenz von Stufen in der Oberfläche der Drähte bestätigen können. Experimentell wurde allerdings bisher eine direkte Verbindung zwischen einer Oberflächenstufe und einer Keimbildung nicht nachgewiesen.

Die Triebkraft für die Keimbildung ist die von außen angelegte Spannung. Im Allgemeinen ist der Querschnitt über die gesamte Drahtlänge homogen. Sieht man von dem Einfluss der Platinschicht ab, welche zu den beiden Fixierpunkten zunimmt, ist eine homogene Spannung entlang des Drahtes zu erwarten. Durch die mechanische Vorbehandlung ging neben den eingebrachten Defekten üblicherweise auch eine Verjüngung des Drahtes einher, so dass in diesen Bereichen eine Spannungskonzentration zu prognostizieren ist. Sieht man sich das Modell von Roos [11] an, so ist die Nukleationsrate sehr sensitiv gegenüber der außen angelegten Spannung. Betrachtet man einen 100nm Draht, der unter einer Spannung steht, die zu einer Nukleationsrate von 1/s für die leading-Partialversetzung in der gesamten Drahtlänge korrespondiert und geht weiter von einer Verjüngung um 5nm aus, was eher einer unteren Grenze entspricht, so 
führt die erhöhte Spannung in dem verjüngten Bereich zu einer Erhöhung der Nukleationsrate um etwa 4 Größenordnungen. Die Ergebnisse dieser Arbeit haben aber gezeigt, dass zwar das Versagen im Allgemeinen in dem verjüngten Bereich erfolgt. Während der Verformung war dagegen entlang des gesamten Drahtes und damit auch im ungestörten Bereich das Auftauchen von Defekten zu beobachten. Dies deckt sich ebenfalls mit Beobachtungen aus der Arbeit von Roos [11], in der eine Verjüngung des Drahtes durch die Bestrahlung mit Gallium-Ionen in einem bestimmten Bereich des Drahtes herbeigeführt wurde. Auch hier konnten Defekte außerhalb des verjüngten Bereiches beobachtet werden. Es müssen also spezielle Keimbildungsstellen entlang des Drahtes vorhanden sein, die zu einer ähnlichen Spannungskonzentration wie in der Verjüngung führen bzw. eine Reduktion der Energiebarriere für die Keimbildung darstellen, so dass trotz geringerer Spannung eine Keimbildung stattfindet.

Ein möglicher Faktor für solche speziellen Keimbildungspunkte scheinen die punktartigen Defekte zu sein, welche bereits vor dem Versuch im Draht vorhanden sind. Wie die Ergebnisse gezeigt haben, stellen diese Defekte einen neuen Aspekt der Keimbildung dar. So konnte eindeutig gezeigt werden, dass sowohl in einkristallinen als auch in verzwillingten Proben die ersten Defekte der Verformung an diesen punktartigen Defekten auftreten. Die Punktdefekte begünstigen folglich die Keimbildung von Versetzungen. Die Art der Keimbildung wird jedoch im Vergleich zu einer ungestörten Stelle nicht verändert, da weiterhin planare Defekte beobachtet wurden und somit von der Keimbildung einer leading-Partialversetzungen auszugehen ist. In der Mehrzahl der Fälle konnte jeweils nur ein Nukleationsevent pro punktartigem Defekt beobachtet werden. Die Keimbildung an sich muss also die Keimbildungsstelle 'verbrauchen' bzw. derart verändern, dass eine weitere Keimbildung an dieser Stelle ungünstiger wird. Weiterhin scheint ein punktartiger Defekt für die Keimbildung nur geringfügig bevorzugt zu sein, da gewöhnlich direkt im Anschluss auch Keimbildungen an Stellen im Draht erfolgen, an denen kein punktartiger Defekt erkennbar war. Generell scheint es eine Abstufung der verschiedenen möglichen Keimstellen zu geben, da die Nukleation immer nach und nach entlang des Drahtes beobachtet werden konnte. Dies entspricht auch der Form der Spannungs-Dehnungs-Kurven, in denen nie ein abrupter Übergang von elastischer zu plastischer Verformung beobachtet worden ist. Ein anderer Grund für einen sukzessiven Übergang von elastischer zu plastischer Verformung könnte darin liegen, dass die Wechselwirkung zwischen Partialversetzungen und Defekten wie 
zum Beispiel Stapelfehler auf dem konjugierten Gleitsystem die beobachtete Verfestigung verursacht. Allerdings muss man zwischen der Nukleation und der Bewegung einer Partialversetzung von separierten Prozessen ausgehen [45]. Da in den allermeisten Fällen die Bewegung der Partialversetzung nicht beobachtet werden konnte, ist davon auszugehen, dass die Nukleation der limitierende Prozess ist. Damit scheint eine Verformungsverfestigung durch Behinderung der Bewegung der Partialversetzung durch planare Defekte auf dem konjugierten Gleitsystem eine untergeordnete Rolle zu spielen. Wahrscheinlicher scheint dagegen zu sein, dass die Keimstellen leicht unterschiedliche Aktivierungsspannungen benötigen und erst nach und nach angesprochen werden. Gleichzeitig impliziert dies, dass mit der Nukleation die Keimstelle abgenutzt wird und nicht wie im Fall einer quellenkontrollierten Verformung viele Versetzungen nacheinander bilden kann, z.B. in Form einer Spiralarm-Quelle [83].

Zusammenfassend lässt sich festhalten, dass die Keimstellen für die leadingPartialversetzungen stark ausgezeichnete Punkte entlang des Drahtes sein müssen. Gleichzeitig müssen diese ausgezeichneten Keimstellen jedoch mit einer gewissen Häufigkeit vorkommen. Dies wird ebenfalls dadurch gestützt, dass die ermittelte Fließspannung auffallend konstant ist. Es wäre denkbar, dass gerade die Kombination aus Oberflächenstufe und punkartigem Defekt als erste Keimstelle dient und anschließend Oberflächenstufen allein als ausgezeichneter Punkt für eine Nukleation ausreicht. Allerdings konnte in dieser Arbeit nicht geklärt werden, was genau diese punktartigen Defekte auszeichnet. Weiterhin bleiben die Gründe ungeklärt, warum sie sich positiv, sei es durch Absenkung der Energiebarriere oder durch eine Spannungskonzentration, auf die Nukleation einer leading-Partialversetzung auswirken.

\subsubsection{Nukleation von twinning-Partialversetzungen}

In allen Proben, also sowohl in einkristallinen als auch verzwillingten Proben, war das Entstehen von Stapelfehlern zu beobachten, was durch die Nukleation einer leading-Partialversetzung erklärbar ist, die in ihrer Spur einen Stapelfehler hinterlässt. Für die weitere Verformung würde man annehmen, dass hauptsächlich die Keimbildung von leading-Partialversetzungen auf benachbarten Ebenen, also von twinningPartialversetzungen, vonstatten geht, da die Energie für eine Zwillingsgrenze kleiner ist als für einen Stapelfehler [87]. So ist auch nach dem Modell von Weinberger et al. [19] eine Verformung durch twinning-Partialversetzungen der erwartete Mechanismus. 
In den Ergebnissen konnte nachgewiesen werden, dass in einkristallinen Drähten Zwillinge mit einer Breite zwischen 3 Atomlagen und über 100nm auftreten und man deshalb in der Tat von der Keimbildung von twinning-Partialversetzungen ausgehen kann. Die Ergebnisse haben aber auch gezeigt, dass es zum Auftauchen von vielen separierten planaren Defekten kommt und das Wachsen von Zwillingen nur nach und nach stattfindet. Die Keimbildung einer twinning-Partialversetzung scheint also im Fall der einkristallinen Drähte nur unter bestimmten Bedingungen abzulaufen. Dies bestätigen auch die Ergebnisse der Versuche mit den vordeformierten Drähten. Planare Defekte, die vorher in den Draht eingebracht wurden, erweisen sich nicht als bevorzugte Orte für die Keimbildung von twinning-Partialversetzungen. Dies scheint im Gegensatz zu MD-Simulationen zu stehen, bei denen ausschließlich die Nukleation von twinningPartialversetzungen an bereits vorhandenen planaren Defekten beobachtet wurde [21].

Im Fall der verzwillingten Drähte ließen sich sowohl während als auch nach der Verformung keine Zwillinge feststellen, sofern man von der Bruchkante absieht. Die Keimbildung der twinning-Partialversetzung scheint für die verzwillingten Drähte also unterdrückt zu sein. Es stellt sich somit die Frage, was verursacht bzw. verhindert die Nukleation einer leading-Partialversetzung auf einer benachbarten Ebene, also einer twinning-Partialversetzung.

\section{Einkristalline Drähte}

In einkristallinen Drähten hat Roos [11] das Auftreten von separierten planaren Defekten durch eine Verteilung von Oberflächenstufen entlang des Drahtes erklärt. Je nachdem ob auf benachbarten Ebenen eines planaren Defektes weitere Stufen vorhanden sind, kommt es nach seinem Modell zu einer Zwillingsbildung. Daneben kann als eine Ursache für das Auftreten von separierten Zwillingen auch die Unterdrückung eines weiteren Wachstums eines bestehenden Zwillings herangezogen werden. Eine Möglichkeit wäre hierbei die Wechselwirkung mit einem planaren Defekt auf dem konjugierten Gleitsystem [21]. Dies ist aber aufgrund der Ergebnisse dieser Arbeit auszuschließen, da häufig planare Defekte beobachtet wurden, bei denen es ohne Wechselwirkung mit dem konjugierten Gleitsystem nicht zu einem Wachstum gekommen ist.

Wie in Abschnitt 2.2.3 beschrieben, haben MD-Simulationen gezeigt, dass es zu einem konkurrierenden Verhalten zwischen einer Nukleation auf benachbarten Ebenen mit Zwillingswachstum einerseits und einer Nukleation auf unabhängigen Ebenen mit 
separierten Stapelfehlern über den Draht verteilt durch mehr oder weniger stark ausgeprägten $\{100\}$-Facetten im Drahtquerschnitt andererseits kommt [29][30]. In den Versuchen dieser Arbeit konnte ebenfalls ein konkurrierendes Verformungsverhalten zwischen leading- und twinning-Partialversetzungen beobachtet werden. Allerdings konnte im Rahmen dieser Arbeit kein Trend festgestellt werden, dass es mit stärker ausgeprägter $\{100\}$-Facettierung auch zu einer ausgeprägteren Zwillingsbildung kommt.

Sofern der Draht stabil befestigt ist, führt die Umorientierung des Drahtes durch die Verzwilligung zu einem Biegemoment über die Zwillingsgrenze herüber, das mit steigender Zwillingsgröße ansteigt. Der Effekt eines solchen Biegemoments führt in der Literatur zu widersprüchlichen Ergebnissen. Lee et al. [22] konstatieren, dass ein solches Biegemoment dazu führe, dass die Schmidfaktoren in dem noch nicht verzwillingten Bereich gegenüber der Zwillingsgrenze bevorzugt würden. So würde ab einer bestimmten Zwillingsgröße die Nukleation auf dem konjugierten Gleitsystem begünstigt und der ursprüngliche Zwilling höre auf zu wachsen. Sedlmayr et al. [21] argumentieren dagegen, dass es ab einer bestimmten Zwillingsgröße durch die Biegemomente zu einem Wechsel von einem Lage-nach-Lage-Wachstum des Zwillings zu einer Stimulation von parallelen Zwillingen vor dem ursprünglichen Zwilling käme, die anschließend zusammenwachsen können und dann erneut einen weiteren Zwilling stimulieren könnten. Eine solche Stimulation von neuen Zwillingen war jedoch in dieser Arbeit in den insitu TEM Untersuchungen nicht zu beobachten. Allerdings ließen sich in den stark verformten Drähten häufig Bereiche erkennen, in denen ein Gleitsystem scheinbar dominiert hat, was möglicherweise ein Indiz für das stimulierte Verhalten sein könnte. Sedlmayr et al. [21] haben ein solches Verhalten aber mit deutlichen Spannungsabfällen in ihren Spannungs-Dehungs-Kurven in Verbindung gebracht. Zwar konnten geringe Spannungsabfälle auch in dieser Arbeit beobachtet werden, allerdings fallen diese mit maximal 20MPa wesentlich kleiner aus und kommen daher nicht als Hinweise für ein stimuliertes Zwillingswachstum in Betracht.

Vergleicht man die Spannungs-Dehnungs-Kurven mit weiteren Kurven von $\langle 110\rangle$ Golddrähten aus der Literatur, so zeigt sich eine gute Übereinstimmung mit einem Teil der Versuche von Sedlmayr et al. [21]. Auch hier hat ein Teil der Drähte nach dem Erreichen der Fließgrenze eine Verformung auf annähernd dem gleichen Spannungsniveau wie der Fließgrenze gezeigt. Auch das Verformungsbild zeigt ein ähnliche Bild mit separierten planaren Defekten entlang des Drahtes, so dass sich ein übereinstimmendes 
Bild ergibt.

Viele weitere Studien zeigen jedoch ein anderes Bild für die Spannungs-DehnungsKurven mit einem drastischen Abfall der Spannung nach Erreichen der Fließspannung [20][72][28][21][4]. Die weitere plastische Verformung erfolgt dann bei Fließspannungen von wenigen 100MPa und gleichzeitig können sehr hohe plastische Dehnungen von über $40 \%$ erreicht werden. Auch das Verformungsbild stellt sich anders dar. So ist statt einer homogenen Verteilung von separierten planaren Defekten auf beiden gleichberechtigten Gleitsystem nur die Aktivierung eines Gleitsystems und das Ausbilden eines einzelnen langen Zwillings sichtbar.

Ein vergleichbares Verhalten, sowohl vom Spannungs-Dehnungs-Verhalten als auch vom Verformungsbild, konnte im Zuge dieser Arbeit nur in einem einzigen Fall festgestellt werden. Dabei handelt es sich gerade um den Draht, bei dem eine fehlerhafte Klebestelle festgestellt worden ist und die Klemme dadurch leicht rotierbar wurde. Auch die Versuchsanordnungen von [20][72][28] zeichnen sich durch einen leicht nachgiebigen Aufbau aus. Es ist also möglich, dass ein solches Verformungsbild bedingt durch den experimentellen Aufbau verursacht wird. Auch Lee et al. [22] betonen die Bedeutung der Randbedingungen des experimentellen Aufbaus für die plastische Verformung bei Nanodrähten. Sie kommen zu dem Schluss, dass Zwillinge stabil wachsen können, solange die Randbedingungen eine laterale Bewegung zulassen und eine Bevorzugung des Gleitsystems des Zwillings erhalten bleibt. In der vorliegenden Arbeit wurde gerade darauf geachtet, einen möglichst stabilen Aufbau zu verwenden und einen uniaxialen Spannungszustand zu gewährleisten. So scheint es nicht weiter verwunderlich, dass in den weiteren Versuchen dieser Arbeit ein solches Verhalten nicht beobachtet werden konnte.

\section{Verzwillingte Drähte}

Im Gegensatz zu einkristallinen Drähten war in verzwillingten Drähten die Ausbildung eines Verformungszwillings nicht nachzuweisen. Dieses Verhalten, also das 'NichtAuftreten' von twinning-Partialversetzungen in verzwillingten Drähten im Gegensatz zu einkristallinen Nanodrähten, deckt sich mit weiteren Studien in der Literatur [88][89][90][91][92]. In den meisten Fällen werden hierbei jedoch fünffach verzwillingte Drähte untersucht [88][89][90], wohingegen Drähte mit einer einzigen Zwillingsgrenze selten Gegenstand von Untersuchungen waren [91][92]. In den meisten dieser Arbeiten 
wird jedoch die Erscheinung, dass es zu keiner Zwillingsbildung kommt, nicht thematisiert.

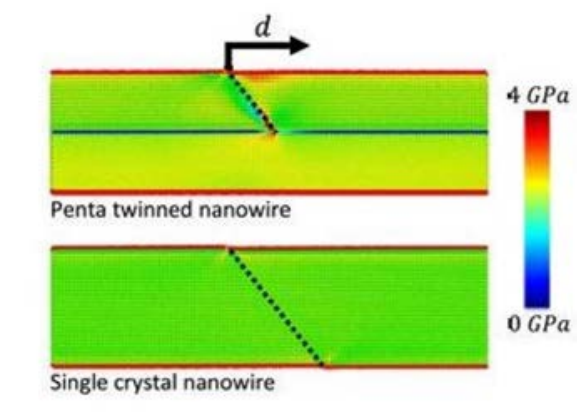

[110]

(a)

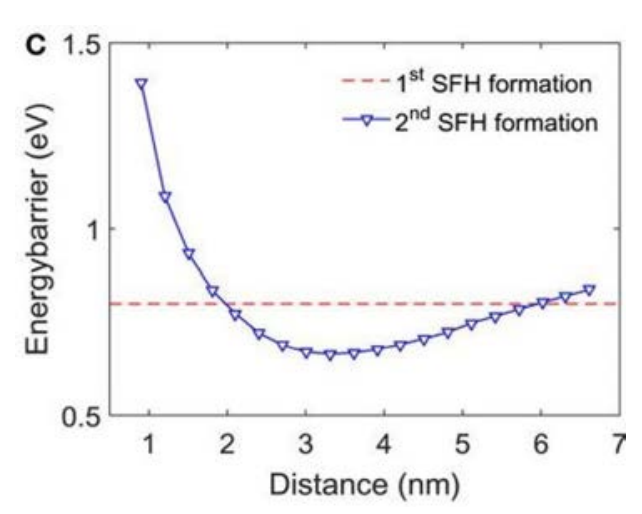

(b)

Abbildung 5.2.: (a) Spannungsfeld eines Stapelfehlers in einem (fünffach)verzwillingten Draht und in einem einkristallinen Draht; (b) Energiebarriere für die Formation eines Stapelfehlers in einem verzwillingten Draht (aus [90])

Lee und Ryu [90] haben in ihren Studien die Energiebarriere für das Auftauchen eines weiteren Stapelfehlers bei der Präsenz eines bereits vorhandenem Stapelfehlers in (fünfach)-verzwillingten Nanodrähten berechnet. Sie stellten dabei fest, dass im Gegensatz zu einkristallinen Drähten der erste Stapelfehler ein elastisches Spannungsfeld erzeugt (siehe Abbildung 5.2 a). In verzwillingten Drähten wird die Energiebarriere für die Nukleation einer weiteren Partialversetzung damit abhängig vom Abstand zum ersten Stapelfehler (siehe Abbildung 5.2 (b)). So ist die Energiebarriere nahe dem ersten Stapelfehler deutlich erhöht, so dass hier die Nukleation einer twinning-Partialversetzung energetisch unwahrscheinlich ist. Geht man davon aus, dass kein relevanter Unterschied zwischen fünffach verzwillingten Drähten und einfach verzwillingten Drähten besteht, so ist ein identischer Effekt auch in den getesteten Golddrähten im Rahmen dieser Arbeit zu erwarten. Allerdings zeigen ihre Berechnungen weiter, dass die Energiebarriere für einen bestimmten Abstand vom ersten Stapelfehler ein Minimum durchläuft (circa 3,5nm in Abbildung 5.2 b)), das unter der Energiebarriere der ersten Partialversetzung liegt. Folglich würde man weitere Stapelfehler immer in einem regelmäßigen Abstand erwarten. Ein solches Verhalten konnte in dieser Arbeit nicht bestätigt werden. Im Gegensatz zu den Drähten in den Simulationen weisen reale Drähte jedoch nicht die gleiche Perfektion auf. Es ist also davon auszugehen, dass in den getesteten Drähten 
die Energiebarriere nicht konstant entlang des Drahtes verläuft, sondern verschieden tiefe Senken aufweist. Das erzeugte Minimum durch den ersten Stapelfehler könnte also im Vergleich zu der Energielandschaft eines realen Drahtes vernachlässigbar sein. Die Erhöhung der Energiebarriere nahe dem ersten Stapelfehler ist in den Berechnungen circa um einen Faktor 10 größer und sollte somit auch noch in den getesteten Drähten sichtbar sein. Tatsächlich lag der minimale Abstand bei circa 10nm, was gut mit den Berechnungen übereinstimmt. Somit lässt sich abschließend festhalten, dass die Berechnungen von Lee und Ryu [90] durchaus eine plausible Erklärung für das fehlende Auftreten von twinning-Partialversetzungen durch eine erhöhte Energiebarriere für die Nukleation liefern.

\subsubsection{Nukleation von trailing-Partialversetzungen}

Die Ergebnisse dieser Arbeit haben an verschiedenen Stellen gezeigt, dass es ebenfalls zur Nukleation von trailing-Partialversetzungen gekommen ist. Zum einen konnte in den TEM-Versuchen beobachtet werden, dass ein große Menge an planaren Defekten mit zunehmender Dehnung wieder verschwand. Hierfür kommen nur zwei Mechanismen in Frage: das Zurücklaufen der leading-Partialversetzung oder eben die Nukleation der trailing-Partialversetzung. Das Zurücklaufen der leading-Partialversetzung kann jedoch ausgeschlossen werden, da die angelegte Spannung einer solchen Bewegung entgegenwirkt und es außerdem zu einer Verringerung der Dehnung führen würde. Es bleibt als mögliche Erklärung folglich nur die Nukleation der trailing-Partialversetzung. Die Zwillingsgrenze scheint hierbei nicht entscheidend zu sein, da das Verschwinden sowohl in einkristallinen als auch verzwillingten Drähten beobachtet wurde. Zum anderen haben die Ergebnisse der REM-Untersuchungen gezeigt, dass nach dem Reißen nicht genug planare Defekte in den Drähten gespeichert sind, um die jeweilige plastische Dehnung allein durch die Nukleation von leading- und twinning-Partialversetzungen erklären zu können. Somit muss es zur Nukleation von trailing-Partialversetzungen gekommen sein, da in einkristallinen Drähten im allgemeinen Fall keine weiteren Defekte und in verzwillingten Drähten nur volle Versetzungen, die wahrscheinlich ebenfalls durch die Nukleation der trailing-Partialversetzung entstanden sind, zu beobachten war.

Weiterhin haben die in-situ TEM Aufnahmen gezeigt, dass es häufig zu einem kollektiven Verschwinden der planaren Defekte in Bereich mit einer hohen Dichte von sich kreuzenden planaren Defekten kommt. Dies kann nur durch eine stimulierte Emis- 
sion von trailing-Partialversetzungen erklärt werden. Dabei ließ sich häufig beobachten, dass Konstrukte aus planaren Defekten der zwei unterschiedlichen Gleitsystemen als Zwischenritt entstehen. Ein ähnliches Szenario konnte auch in MD-Simulationen beobachtet werden [21], ohne dass an dieser Steller weiter darauf eingegangen worden ist. Es liegt also nahe, dass die stimulierte Emission durch eine Wechselwirkung von Defekten der beiden Gleitsysteme entsteht. Wie der genaue Mechanismus der stimulierten Emission sich darstellt, konnte jedoch nicht aufgeklärt werden.

Obwohl die trailing-Partialversetzung den Vorteil durch den Abbau der Energie für den Stapelfehler hat, wird eine Nukleation der trailing-Partialversetzung für eine Zugververformung in $\langle 110\rangle$-Richtung nicht erwartet, da der Schmidfaktor nur die Hälfte im Vergleich zu einer leading- bzw. twinning-Partialversetzung beträgt $(m=0,23)$. Mit diesem Argument wird in den meisten Studien die Nukleation einer trailingPartialversetzung ausgeschlossen [21][22][92]. So sagt auch das Modell von Roos eine experimentell beobachtbare Nukleationsrate der trailing-Partialversetzung erst ab Spannungen voraus, die deutlich über den Spannungen für die Nukleation einer twinningoder leading-Partialversetzung liegen. Ebenso sagt das Modell von Weinberger et al. [19], das die generalisierte Stapelfehlenergie berücksichtigt (siehe Kapitel 2.2.2), keine Nukleation von trailing-Partialversetzungen voraus. Allerdings wurde bereits in den Arbeiten von Roos [11] und Erichsen [56] das Verschwinden von planaren Defekten beschrieben und auf die Nukleation von trailing-Partialversetzungen zurückgeführt. In diesen Arbeiten ist aber davon ausgegangen worden, dass es sich dabei nur um Ausnahmefälle handelt, die durch vermeintliche lokale Spannungskonzentrationen initiiert werden. Ebenso konnte in einer Studie an Silber-Nanodrähten das Verschwinden von Stapelfehlern beobachtet werden [91]. Dies wurde ebenfalls auf die Nukleation von trailing-Partialversetzungen zurückgeführt. Eine Begründung, weshalb es zu einer Nukleation von trailing-Partialversetzungen kommt, wurde jedoch nicht geliefert.

Als mögliche Erklärungen für die Nukleation der trailing-Partialversetzungen kommen folgende Ansätze in Betracht:

- Spezielle Oberflächenkonfigurationen

- Kinetische Energie von Versetzungen

- Querschnittsform der Drähte

Oberflächendefekte oder -unregelmäßigkeiten stellen bevorzugte Stellen für die Nukleation von Versetzungen dar [40][85][33][34][86]. Will man die Nukleation der trailing- 
Partialversetzung mit einer bestimmten Oberflächenstruktur erklären, so müsste diese Struktur lediglich die Nukleation der trailing- und nicht die der leading- bzw. twinningPartialversetzung begünstigen. Einer der Gründe für die bevorzugte Nukleation an Oberflächendefekten sind die auftretenden Spannungskonzentrationen. Denkbar wäre beispielsweise, dass durch das Gleiten der leading-Partialversetzung eine spezielle Oberflächenstruktur wie eine Kinke in einer Oberflächenstufe entsteht, die eine bevorzugte Spannungskonzentration für die Nukleation einer trailing-Partialversetzung darstellt. Hirel et al. [33] konnten dagegen in ihren Simulationen feststellen, dass eine solche Kinke weder ein ausgezeichneter Punkt für eine Nukleation ist, noch einen besonderen Einfluss auf die Nukleationsspannung der Versetzungen hat oder die Art der Nukleation verändert. Es scheint also keinen Grund dafür zu geben, weshalb sich die Spannungskonzentration eines Oberflächendefektes bevorzugt auf die Nukleation einer trailing-Partialversetzung auswirken sollte.

Neben der Spannungskonzentration ist ein zweiter Grund für die bevorzugte Nukleation an Oberflächendefekten der Energiegewinn durch die Reduktion der Oberflächenstufe. Je nach Orientierung des Burgersvektors in Hinblick auf die Oberfläche wird die Stufe zwischen $1 / 3$ und 2/3 bei der Nukleation einer Partialversetzung reduziert. In den Modellen von Hirel et al. [34] und Roos [11] wird jeweils davon ausgegangen, dass die leading-Partialversetzung die Oberflächestufe um 2/3 reduziert und die trailing-Partialversetzung um 1/3. Ebenso könnte aber die Stufe so angeordnet sein, dass beide Werte vertauscht sind. Dies würde in dem Modell von Roos die beiden Nukleationsraten der leading- und trailing-Partialversetzung enger zusammenrücken lassen, allerdings nicht zu einem Wechsel der Reihenfolge von leading- und trailingPartialversetzung führen. Somit scheint auch die Reduktion einer Oberflächenstufe als mögliche Erklärung für die Nukleation einer trailing-Partialversetzung untauglich zu sein.

Beim Durchlaufen des Drahtes besitzt eine Partialversetzung analog zur Mechanik eine kinetische Energie $E_{k i n}=1 / 2 m_{d} v_{d}^{2}$ mit der Masse $m_{d}$ und der Geschwindigkeit $v_{d}$ einer Versetzung. Bei einer von außen wirkenden Scherspannung kann die Geschwindigkeit einer Versetzung über $v_{d}=\tau / B \cdot e^{-20 \tau_{r} / \tau}$ abgeschätzt werden [75][93], wobei für Gold der Koeffizient $B \approx 3 \cdot 10^{5} \frac{\mathrm{Pa} \cdot \mathrm{s}}{\mathrm{m}}$ und der Reibungswiderstand für einen 100nm Draht circa $\tau_{r} \approx 100 \mathrm{MPa}$ beträgt [75]. Nimmt man einen Wert für die Zugspannung von $\sigma \approx 1300 M P a$, die alle Drähte erreichen, so wirkt auf die Partialversetzung bei 
einem Schmidfaktor von $m=0,5$ eine Scherspannung von $\tau=650 M P a$. Damit ergibt sich eine Geschwindigkeit von $v_{d} \approx 100 \mathrm{~m} / \mathrm{s}$, die wesentlich kleiner ist als als die Schallgeschwindigkeit in Gold $\left(v_{d}<<c_{t}=3280 \mathrm{~m} / \mathrm{s}\right.$ [94]) Die Masse lässt sich dann nach [95] zu $m_{v} \approx \rho b^{3}$ pro Länge b der Versetzung nähern, also einer Atommasse pro Netzebene. Für eine 100nm lange Versetzung ergibt sich dann eine Masse von $m_{d} \approx 1,6 \cdot 10^{-22} \mathrm{~kg}$. Somit erhält man für die kinetische Energie einer Versetzung

$$
E_{k i n}=1 / 2 m_{d} \cdot v_{d}^{2} \approx 5 e V
$$

Damit liegt die kinetische Energie im gleichen Größenbereich wie die Energiebarriere für die Nukleation einer Partialversetzung bei einer Spannung von $\sigma=1,7 G P a$ entsprechend dem Modell von Jennings et al. [35]. Bei einem kompletten Energietransfer könnte die kinetische Energie also für die Nukleation einer Versetzung ausreichen. Für höhere Spannungen nimmt die kinetische Energie durch eine höhere Geschwindigkeit weiter zu und die Aktivierungsenergie ab, so dass die Wahrscheinlichkeit einer Nukleation weiter zunimmt. Es bleibt allerdings die Frage, in welche Kanäle die kinetische Energie der Versetzung geleitet wird. So scheint es wahrscheinlicher, dass ein Großteil der Energie in Form thermischer Anregung dissipiert und damit nur ein sehr geringer Anteil für die Nukleation von einer neuen Versetzung genutzt werden kann. Sollte dieser Anteil an kinetischer Energie doch für die Nukleation einer Versetzung ausreichen, stellt sich weiterhin die Frage, warum es ausgerechnet zur Nukleation einer trailing-Partialveretzung kommen sollte. Man kann folglich die kinetische Energie einer Versetzung als mögliche Erklärung für die Nukleation einer trailing-Partialversetzung nicht von vornherein ausschließen. Allerdings scheint diese Möglichkeit sehr unwahrscheinlich.

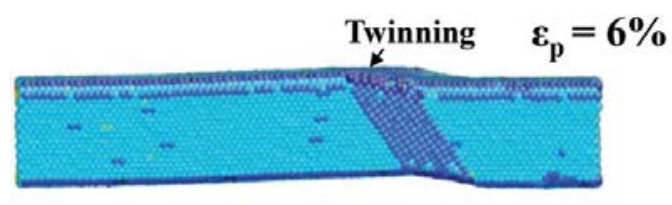

(a)

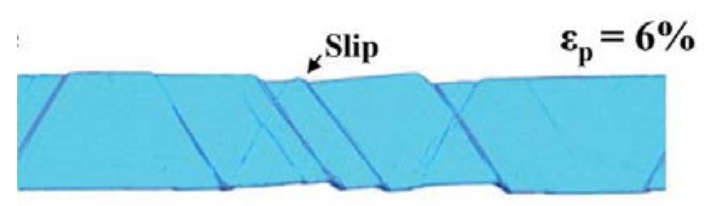

(b)

Abbildung 5.3.: MD-Simulationen zur Verformung von Gold-Drähten mit unterschiedlichem Aspektverhältnis (aus [28]): (a) Verformung via twinning bei einem 1:1-Verhältnis; (b) Verformung via Slip (volle Versetzungen) bei einem 10:1-Verhätnis 
In der Literatur wird in einer kombinierten Studie aus experimentellen Zugversuchen und MD-Simulationen beschrieben, dass es einen Übergang von einer Verformung durch twinning-Partialversetzungen in Gold-Nanodrähten mit homogenem Querschnitt zu einer Verformung durch volle Versetzungen (leading-Partialversetzung gefolgt von einer trailing-Partialversetzung) in Drähten mit einem hohen Aspektverhältnis im Drahtquerschnitt kommt [28] (siehe Abbildung 5.3). Von einem ähnlichen Zusammenhang hat ebenfalls Sedlmayr [60] berichtet, der in Drähten mit hohem Aspektverhältnis im Gegensatz zu Drähten mit homogenerem Querschnitt ein Auftreten von großen Zwillingen nicht entdecken konnte. Auch in den Versuchen dieser Arbeit gibt es Hinweise darauf, dass die Querschnittsform ein Rolle für die Nukleation der trailing-Partialversetzung spielt. So ließen sich die Abgleitung entlang einer kristallographischen Ebene häufiger in Drähten mit einem hohen Aspektverhältnis beobachten, was einen Hinweis auf die Aktivität von vollen Versetzungen und damit auch auf die Nukleation von trailingPartialversetzungen gibt. Darüber hinaus konnte eine umfangreiche Speicherung von Stapelfehlern und Zwillingen ausschließlich in kleinen Drähten mit homogenem Querschnitt beobachtet werden.

Die Bedeutung der Querschnittsform wird von Hwang et al. [28] auf den Einfluss der Oberflächenenergie zurückgeführt. Demnach ist es bei Drähten mit einem hohen Aspektverhältnis energetisch ungünstiger eine Reorientierung der $\{111\}$-Seitenflächen $\left(\gamma_{111}=1,52 \mathrm{~J} / \mathrm{m}^{2}\right)$ zu $\{100\}$-Seitenflächen $\left(\gamma_{100}=1,80 \mathrm{~J} / \mathrm{m}^{2}\right)$ bei der Zwillingsbildung zu durchlaufen. Durch die Nukleation der trailing-Partialversetzung entsteht stattdessen nur eine kleine Oberflächenspur auf der $\{111\}$-Seitenfläche, die weniger Energie beansprucht als die Reorientierung.

Um diesen Aspekt der Oberflächenreorientierung in das Modell der klassischen Keimbildung einzuarbeiten, kann Gleichung 2.7 um einen Oberflächenterm $E_{\text {Surface }}$ erweitert werden:

$$
E=E_{d}+E_{\text {relax }}+E_{s}+E_{\gamma}+E_{\text {Surface }}
$$

Der Oberflächenterm lässt sich durch $E_{\text {Surface }}= \pm 2 R b \Delta \gamma$ abschätzen, wobei $\Delta \gamma=$ $\gamma_{111}-\gamma_{100}$ der Unterschied zwischen den Oberflächenenergie der beiden möglichen Facetten ist. Für eine leading- und twinning-Partialversetzung muss diese Energie aufgebracht werden, für eine trailing-Partialversetzung wird diese Energie wieder zurückgewonnen. Diese Erweiterung führt schließlich dazu, dass sich die Reihenfolge der Par- 
tialversetzungen ändert. So benötigt die Nukleation einer trailing-Partialversetzung die kleinste Spannung, um eine experimentell sichtbare Rate zu erzielen. Erst anschließend folgen die twinning- und leading-Partialversetzungen. Es ist allerdings fraglich, ob man bereits ab einer durchgelaufen Partialversetzung von einer Reorientierung der Drahtoberfläche ausgehen kann. Weiterhin lässt sich die klassische Nukleationstheorie nur bedingt mit den MD-Simulation von Hwang et al. [28] in Einklang bringen. Betrachtet man den typischen Keimradius für die Nukleation der Partialversetzungen, so liegt dieser in allen Fällen deutlich unter der Facettenlänge der Drähte. Ein Versetzungskeim sollte also sowohl bei Drähten mit homogenem Querschnitt als auch bei Drähten mit hohem Aspektverhältnis identisch sein und entsprechend die Querschnittsform keinen Einfluss auf das Nukleationsverhalten haben. Der Einfluss der Querschnittsform und der Oberfläche lässt sich also nicht ohne weiteres in das Modell einer Oberflächenkeimbildung integrieren.

\subsection{Wechselwirkung von Partialversetzungen mit weiteren Defekten}

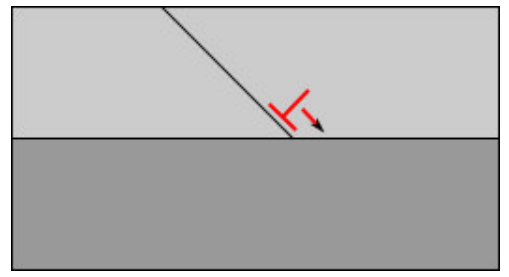

(a)

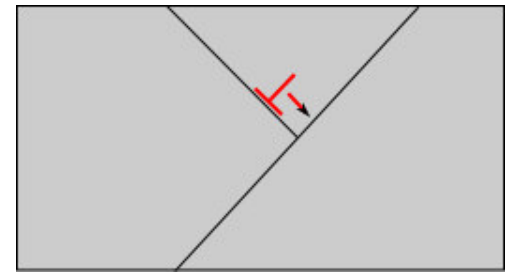

(b)

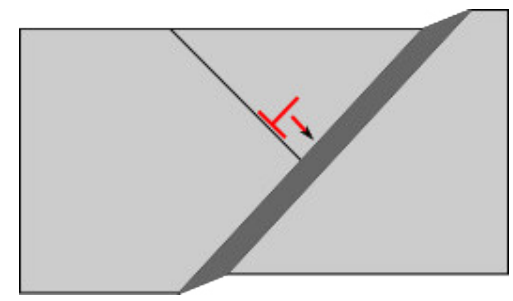

(c)

Abbildung 5.4.: Wechselwirkung einer leading-Partialversetzung mit einer longitudinalen Zwillingsgrenze (a), einem Stapelfehler auf dem konjugierten Gleitsystem (b) oder einem Verformungszwilling auf dem konjugierten Gleitsystem (c)

Die Ergebnisse dieser Arbeit haben gezeigt, dass es während der Verformung der Nanodrähte zu verschiedenen Wechselwirkungen zwischen einer Partialversetzung und Defekten im Draht kommen muss. Hierbei handelt es sich hauptsächlich um drei Szenarien: Die Wechselwirkung mit einer longitudinalen Zwillingsgrenze (Abbildung 5.4 (a)), die Wechselwirkung mit einem Stapelfehler auf dem konjugierten Gleitsystem (Abbildung 5.4 (b)) und die Wechselwirkung mit einem Verformungszwilling auf der 
konjugierten Ebene (Abbildung 5.4 (c)), wobei sich letzteres Szenario nur in der Anzahl der Ebenen zu der Wechselwirkung mit einem Stapelfehler unterscheidet.

\section{Wechselwirkung mit longitudinaler Zwillingsgrenze}

Die Wechselwirkung einer Versetzung mit einer Zwillingsgrenze wurde in der Literatur bereits ausführlich untersucht [96][97][98][99]. Grundsätzlich zeigen alle diese Arbeiten, dass eine Zwillingsgrenze ein Hindernis für die Bewegung einer Partialversetzung darstellt. Die Partialversetzung wird demnach an der Zwillingsgrenze blockiert und am Weiterlaufen gehindert. Das Blockieren der Partialversetzung konnte bereits in anderen Experimenten von Nanodrähten mit longitudinalen Zwillingsgrenzen beobachtet werden [89][100] und hat sich auch in dieser Arbeit bestätigt (siehe Abbildung 4.26). Bei weiterer Verformung kommen neben der Möglichkeit, dass die Partialversetzung an der Zwillingsgrenze verbleibt, zwei weitere Möglichkeiten in Betracht: 1. die Partialversetzung gleitet quer in die Zwillingsgrenze, 2. die Partialversetzung transmittiert in den Zwilling. Zusammen mit der im Zugversuch gegebenen Geometrie und Lage der longitudinalen Zwillingsgrenze scheint die erste Möglichkeit unwahrscheinlich, da auf die Zwillingsgrenze keine Scherspannung wirkt. Im Falle einer Transmission in den Zwilling existiert in dem Zwilling kein äquivalentes Gleitsystem für die Partialversetzung. Die Partialveretzung muss folglich eine Reaktion durchlaufen und das Gleitsystem wechseln, wodurch an der Zwillingsgrenze eine Art immobile Stairrod-Versetzung zurückbliebe. Die Versetzungreaktion sähe dann wie folgt aus:

$$
1 / 6[\overline{12} 1] \rightarrow 1 / 6[\overline{211}]^{T}+2 / 9[\overline{111}]
$$

' $T$ ' gibt dabei an, dass sich diese Versetzung im verzwillingten Bereich befindet. Bei der Stairrod-Versetzung (2/9[111]) könnte es sich gerade um die Art der Versetzung handeln, die häufig nach den Zugversuchen in den Drähten zu beobachten ist (siehe Abbildung 4.35). Allerdings müsste die Stairrod-Versetzung dann in beiden Zwillingshälften jeweils mit einem Stapelfehler verknüpft sein. Diese Kombination konnte jedoch nicht festgestellt werden. Wu et al. [98] berichten im Rahmen ihrer Simulationen, dass die Stairrod-Versetzung weiter dissoziieren könnte in jeweils eine trailing-Partialversetzung 
in beiden Zwillingen entsprechend folgender Reaktion:

$$
2 / 9[\overline{111}] \rightarrow 1 / 6[1 \overline{1} 2]+1 / 6[112]^{T}
$$

Diese trailing-Partialversetzungen würden durch ihr Gleiten die beiden Stapelfehler wieder annihilieren und den Draht und auch die Zwillingsgrenze defektfrei zurücklassen. Dieses Szenario erscheint durchaus plausibel. Allerdings konnte in allen verzwillingten Drähten die Speicherung von Versetzungen beobachtet werden. Dies wäre mit der o.a. Reaktion nicht erklärbar. Sollte das obige Szenario dennoch eintreten, müssten die Versetzungen durch einen anderen Prozess eingebracht werden (siehe auch Abschnitt $5.4)$.

\section{Wechselwirkung mit einem Stapelfehler}

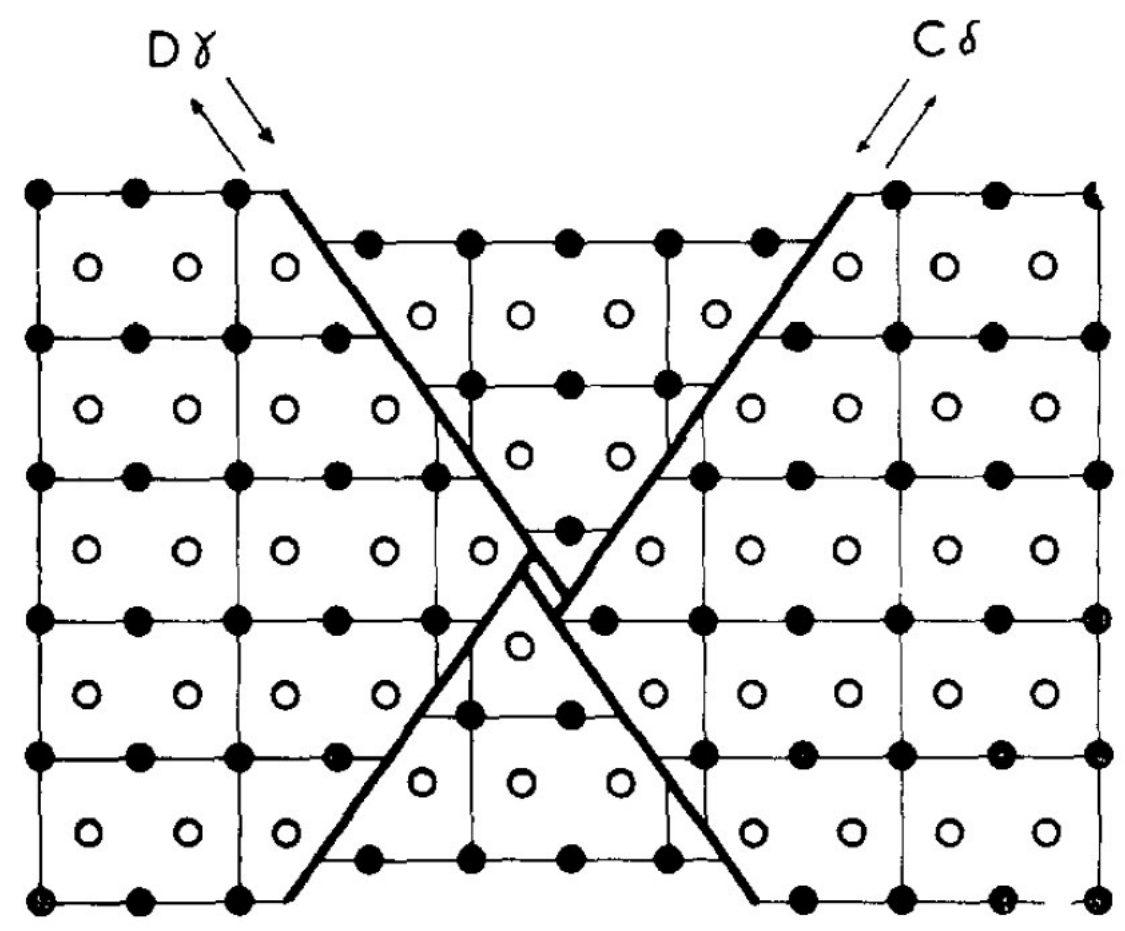

Abbildung 5.5.: Schematische Darstellung einer Kreuzung zweier Stapelfehlern (aus [101])

Das Kreuzen von Stapelfehlern wurde bereits mehrfach durch TEM-Aufnahmen beobachtet [101][102]. Bei einer Kreuzung muss die gleitende Partialversetzung einen kleinen Kletterprozess durchführen, da die Gleitebene wegen des Stapelfehlers eine kleine Stufe aufweist. Das Resultat sind zwei sich kreuzende Stapelfehler wie in Abbildung 
5.5 dargestellt (aus [101]). Am Ort des Kreuzens entsteht dabei eine kleine Gitterausdehnung, dich sich auch als Stairrod-Versetzungsdipol interpretieren lässt [103]. Die Energie, die für die Ausdehnung aufgebracht werden muss, kann zu 0,2eV pro Atomebene abgeschätzt werden [101]. Mit diesem Wert kommt Rajan [102] zu dem Ergebnis, dass ein Stapelfehler in der Tat ein Hindernis für die Bewegung einer Partialversetzung darstellt. Diese Barriere ist jedoch nicht besonders groß. Dies deckt sich mit den Beobachtungen in dieser Arbeit, bei denen häufig das Kreuzen von Stapelfehlern festzustellen war, wobei deren Entstehen sich üblicherweise innerhalb eines Kameraframes abspielte, was als Hinweis auf eine geringe Barriere gewertet werden kann. In Fällen, in denen Partialversetzungen scheinbar von planaren Defekten aufgehalten wurden, handelt es sich möglicherweise um kleine Nanozwillinge, die wahrscheinlich eine größere Barriere als Stapelfehler für die Bewegung der Partialversetzung darstellen.

\section{Wechselwirkung mit einem Verformungszwilling}

Wie die longitudinale Zwillingsgrenze stellt auch ein Zwilling auf dem konjugierten Gleitsystem ein Hindernis für die Bewegung einer Partialversetzung dar [98], so dass auch hier das Blockieren der Partialversetzung zu erwarten ist. Anschließend stehen neben dem Verbleib an der Zwillingsgrenze erneut die zwei Optionen zum einen Quergleiten, zum anderen Transmission zur Verfügung. Im Gegensatz zur longitudinalen Zwillingsgrenze liegt der Burgersvektor in diesem Fall jedoch unter 90 zum Linienelement der Versetzung, wodurch sich andere Versetzungsreaktionen ergeben. Am wahrscheinlichsten scheint für diese Geometrie das Quergleiten in die Zwillingsgrenze zu sein, da die ankommende leading-Partialversetzung äquivalent zu einer leadingPartialversetzung auf der Zwillingsgrenze ist und somit keine Energie-Barriere für diese Versetzungsreaktion vorhanden ist [99]. Dieser Prozess führt dann dazu, dass der Verformungszwilling um eine Atomlage wächst oder schrumpft. Tatsächlich konnte ein solcher Fall in den TEM-Aufnahmen beobachtet werden. Eine Transmission in den Zwilling erscheint dagegen eher unwahrscheinlich, da bei dieser Reaktion Versetzungen auftreten, deren Burgersvektor nur einen Bruchteil von einer Partialversetzung aufweist und damit nicht zu stabilen oder metastabilen Atomkonfigurationen führt [99]. Die Energiebarriere für eine solche Versetzungsreaktion ist entsprechend groß [99]. Allerdings konnte in den Ergebnissen eindeutig gezeigt werden, dass eine Partialversetzung zunächst von einem planaren Defekt auf dem konjugierten Gleitsystem blockiert 
wurde und erst danach den gesamten Draht durchläuft. Geht man davon aus, dass nur Zwillinge eine merkliche Barriere für die Bewegung einer Partialversetzung darstellen, muss es also beim Durchlaufen der Partialversetzung doch zu einer solch ungünstigen Versetzungsreaktion gekommen sein. Denkbar wäre, dass diese Art der Reaktion nur bis zu einer bestimmten Zwillingsbreite möglich ist. Man könnte sich vorstellen, dass ähnlich wie im Fall von zwei kreuzenden Stapelfehlern eine Art Versetzungsdipol an den beiden Grenzen entsteht, der eine solche Reaktion ermöglicht.

\subsection{Speicherung von Versetzungen}

Wie in den Versuchsergebnissen eindeutig zu Tage trat, kommt es im Zuge der Verformung zur Entstehung und zur Speicherung von Versetzungen in verzwillingten Drähten. Da Versetzungen nur in Ausnahmefällen und mit zu vernachlässigender Häufigkeit in einkristallinen Drähten auftraten, muss die Zwillingsgrenze eine ausschlaggebende Rolle für die Speicherung von Versetzungen spielen. Für das Auftreten der Versetzungen kommen demnach zwei Möglichkeiten in Betracht:

(1) Es entsteht eine Versetzungsreaktion der leading-Partialversetzung an der Zwillingsgrenze, in deren Folge sich eine Versetzungen ausbildet, die im Draht gespeichert wird. Wie bereits im Abschnitt 5.3 beschrieben, gibt es verschiedene Möglichkeiten für Wechselwirkungen einer Partialversetzung mit einer longitudinalen Zwillingsgrenze. Bei der in Frage kommenden Reaktion (siehe Abschnitt 5.3) bliebe jedoch keine Versetzung im Draht zurück. Die Reaktion mit der Zwillingsgrenze kommt somit als mögliche Erklärung nicht in Betracht.

(2) Im Gegensatz zu einkristallinen Drähten kann die leading-Partialversetzung nicht ohne weiteres einen verzwillingten Draht durchlaufen, sondern muss die Zwillingsgrenze überwinden. Dabei kann die Partialversetzung aufgehalten werden, wie es auch in den Ergebnissen festgestellt werden konnte, und wäre somit noch im Draht vorhanden. Kommt es nun zur Nukleation einer trailing-Partialversetzung, kann diese mit der aufgestauten leading-Partialversetzung zu einer vollen Versetzung rekombinieren. Da in verzwillingten Drähten ebenfalls das Verschwinden von planaren Defekten beobachtet werden konnte, scheint die Nukleation einer trailing-Partialversetzung durchaus möglich und damit eine plausible Erklärung für das Auftreten von Versetzungen zu sein. Allerdings würde man mit diesem Mechanismus die Versetzungen lediglich auf 
den $\{111\}$-Ebenen erwarten, auf denen die größte Scherspannung wirkt und damit auch die Nukleation der leading- und trailing-Partialversetzung stattfindet. Wie beispielsweise in Abbildung $4.35 \mathrm{zu}$ sehen ist, liegen die Versetzungen aber auch noch auf anderen Ebenen. Es muss also zu einem Quergleiten oder einer Reaktion mit der Zwillingsgrenze gekommen sein, bei der die Versetzung auf eine andere Ebene gekommen ist. Ein Quergleiten scheint eher zweifelhaft, da auf die übrigen $\{111\}$-Ebenen keine Scherspannung wirkt. Im Gegensatz zu einer leading-Partialversetzung existieren für eine volle Versetzung Möglichkeiten für eine Reaktion an der Zwillingsgrenze, in deren Folge kein Stapelfehler entsteht. Eine mögliche Reaktion wäre eine Transmission der vollen Versetzung in den verzwillingten Teil, wobei ein Versetzungssegment auf der Zwillingsgrenze zurückgelassen wird. Die Versetzungsreaktion beginnend mit der Rekombination der beiden Partialversetzungen lautet hierbei wie folgt [97]:

$$
1 / 6[112]+1 / 6[2 \overline{1} 1] \rightarrow 1 / 2[101] \rightarrow 1 / 2[101]^{T}+1 / 2[\overline{11} 2]
$$

Die Versetzung 1/2[101 $]^{T}$ kann nun einfach in dem verzwillingten Bereich weitergleiten und den Draht durch die Oberfläche wieder verlassen. Die Versetzung 1/2[112] bleibt auf der Zwillingsgrenze zurück. Sie entspricht vom Burgersvektor her der in den Ergebnissen in Abbildung 4.35 gezeigten Versetzung. Um ihre Linienenergie möglichst gering zu halten, ist es wahrscheinlich, dass die Versetzung eine möglichst gerade Konfiguration einnimmt, wie in den Versuchen häufig zu beobachten war. Somit scheint die Nukleation einer trailing-Partialversetzung mit anschließender Rekombination mit einer geblockten leading-Partialversetzung und Reaktion mit der Zwillingsgrenze eine hinreichende Erklärung für das Auftreten von Versetzungen in verzwillingten Drähten $\mathrm{zu}$ sein.

\subsection{Bruchverhalten}

Wie die Ergebnisse gezeigt haben, versagen einkristalline Drähte im Allgemeinen in einem verzwillingten Bereich. Dies deckt sich mit den bisherigen Ergebnissen von Roos [11] und Erichsen [56]. Sedlmayr et al. [21] berichten jedoch davon, dass die Drähte an Stellen versagen, an denen sich planare Defekte der beiden aktiven Gleitsystemen treffen. Dieses Verhalten ließ sich aber im Rahmen dieser Arbeit nicht verifizieren.

Innerhalb eines Zwillings erfolgt das Versagen letztlich durch Necking oder Ab- 
scherung entlang einer Ebene(oder einer Mischung aus beiden), wobei Drähte mit homogenem Querschnitt eher zu einem Necking tendieren und Drähte mit einem hohen Aspektverhältnis eher zu einem Abscheren neigen. Dies mag geometrische Gründe haben. Bei plattenähnlichen Strukturen tritt Necking nur entlang der dünnen Richtung auf ohne eine deutliche Kontraktion in der Breite, was dann letztlich zu einer Abscherung führt [28][104]. In beiden Fällen, Necking und Abscherung, ist zu erwarten, dass das Versagen durch die Bewegung von vollen Versetzungen erfolgt. Im verzwillingten Teil ist der Draht in eine $\langle 100\rangle$-Richtung umorientiert. Für eine solche Orientierung hat eine volle Versetzung den höchsten Schmidfaktor $(m=0,408)$, wohingegen die leadingPartialversetzung nur einen Schmidfaktor von $\mathrm{m}=0,236$ aufweist. Partialversetzungen und damit Stapelfehler und Zwillinge sind also in dieser Geometrie nicht zu erwarten. Damit stimmen die Ergebnisse dieser Arbeit mit anderen in der Literatur beschriebenen Experimenten und Simulationen überein, die ebenfalls ein Versagen in dem reorientierten Zwillingsbereich zeigen [18][20].

\subsubsection{Plastische Dehnung bis zum Versagen}

Die plastische Dehnung bis zum Bruch zeigt eine große Streuung von 0, 6\% bis 15, 4\% innerhalb der Drähte. Dies stimmt mit weiteren Studien in der Literatur überein [21][60]. Auch hier konnten innerhalb eines Probensets maximale Dehnungen zwischen 2,5\% $(\approx 0,5 \%$ plastische Dehnung) und $12 \%(\approx 10 \%$ plastische Dehnung) gemessen werden. Andere Studien zeigen dagegen, dass Gold-Nanodrähte plastische Dehnungen bis über 40\% erreichen können [20][72][22][28]. In diesen Studien durchläuft der Draht nahezu komplettes twinning auf einem Gleitsystem entlang seiner Länge. Allerdings zeichnen sich diese Experimente durch einen besonders nachgiebigen Versuchsaufbau insbesondere in lateraler Richtung aus, so dass Biegemomente durch das twinning leichter ausgeglichen werden können. Hwang et al. [28] gehen davon aus, dass die Möglichkeit einer Zwillingsbildung die Höhe der plastischen Dehnung bestimmt. Drähte, die durch Abgleitung durch Bewegung von vollen Versetzungen (leading- + trailingPartialversetzung) verformen, weisen kleine plastische Dehnungen auf. Drähte welche via twinning verformen, können hohe plastische Dehnungen erreichen. Zu einem ähnlichen Ergebnis kommen Gao et al. [105]. In deren Simulationen erreichen einkristalline Drähte durch twinning sehr hohe plastische Dehnungen, wohingegen verzwillingte Drähte, bei denen der twinning Mechanismus nicht möglich ist, früher versagen. Ein 
solches Verhalten ließ sich in den Versuchen zu dieser Arbeit nicht bestätigen. So konnte kein Unterschied für die plastische Dehnung zwischen einkristallinen Drähten und verzwillingten Drähten, die nicht durch twinning verformen, erkannt werden.

In den Ergebnissen dieser Arbeit konnte eher festgestellt werden, dass die Art der Aktuatorsteuerung und damit indirekt die Dehnrate einen möglichen Einfluss auf die maximale plastische Dehnung hat. Dies könnte auch erklären, warum in der Literatur große Unterschiede für die plastische Dehnung existieren, da in den jeweiligen Aufbauten sowohl die Steuerung als auch die Dehnrate sich deutlich voneinander unterscheiden. Die Ergebnisse dieser Arbeit haben gezeigt, dass eine kontinuierliche Erhöhung der Piezo-Spannung größere plastische Dehnungen ermöglicht als eine ruckartige Steuerung des Aktuators. Dabei scheint innerhalb der ruckartigen Steuerung die Schrittweite keine entscheidende Rolle zu spielen und sowohl bei großen (per Hand) als auch bei kleinen (per Computer) Schrittweiten, die auch im Bereich von dem Burgersvektor einer Versetzung liegen, zu einem frühzeitigem Versagen der Drähte zu führen. Es bleibt also unklar, warum gerade eine ruckartige Bewegung zu einem frühzeitigem Versagen führt. Eine Möglichkeit, diese Erscheinung zu erklären ist, dass die ruckartige Bewegung elastische Wellen in den Draht einbringt. Diese könnten mit bereits entstanden Defekten im Draht wechselwirken. Dabei käme es dann zu einer stark lokalisierten Verformung bei dem bereits vorhandenen Defekt, in dessen Folge das Versagen des Drahtes eintritt.

\subsubsection{Verschwinden von planaren Defekten beim Reißen}

Wie die Ergebnisse eindeutig gezeigt haben, kommt es beim Reißen der Drähte ebenfalls zum Abbau von planaren Defekten ohne das Zurücklassen eines Defektes im Draht. Dieses Verhalten wurde sowohl in einkristallinen Drähten (siehe Abbildung 4.20) als auch in verzwillingten Proben beobachtet (siehe Abbildung 4.37).

Bei verzwillingten Drähten kann die leading-Partialversetzung, die den Stapelfehler erzeugt hat, von der Zwillingsgrenze am Weiterlaufen gehindert und damit im Draht gespeichert werden. Beim Reißen entfällt die Spannung, die die Partialversetzung gegen die Zwillingsgrenze gedrückt hat. Die leading-Partialversetzung wird nun auf der einen Seite von der Zwillingsgrenze abgestoßen und gleichzeitig treibt die Stapelfehlerenergie die Partialversetzung zurück, so dass die leading-Partialversetzung den Draht auf ihrem ursprünglichen Weg wieder verlässt und dabei den Stapelfehler annihiliert (siehe Abbildung 5.6). Auf die vorhandenen Versetzungen in den verzwillingten Drähten wirkt 


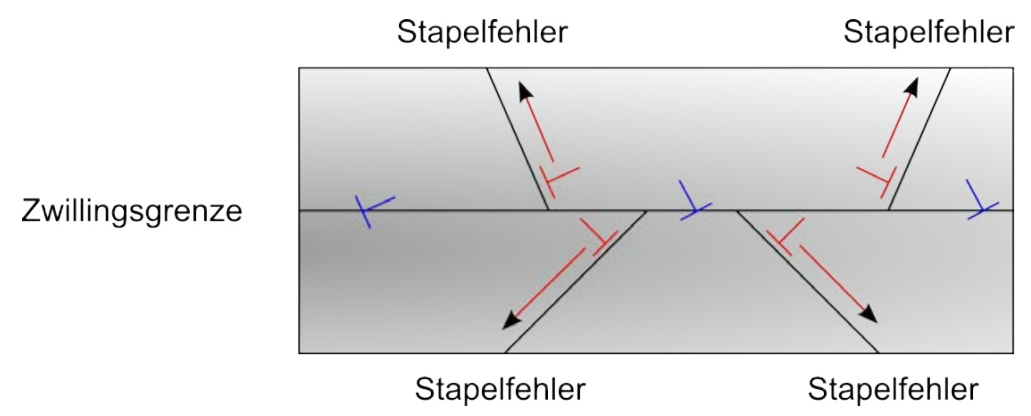

Abbildung 5.6.: Schematische Zeichnung zum Verschwinden von planaren Defekten beim Reißen im Fall eines verzwillingten Drahtes: Die an der Zwillingsgrenze aufgestauten leading-Partialversetzungen (rot) werden durch die Zwillingsrenze und den Stapelfehler aus dem Draht herausgedrückt, wobei der Stapelfehler wieder zurückgebildet wird. Auf volle Versetzungen (blau) wirkt dagegen keine Kraft und sie verbleiben im Draht.

dagegen keine Kraft und sie bleiben im Draht gespeichert. Ein identisches Verhalten konnte auch beim Entlasten der verzwillingten Drähte festgestellt werden. Hierdurch bestätigt sich, dass das Abnehmen der von außen angelegten Spannung für das Zurücklaufen verantwortlich ist. Dieses Zurücklaufen von Partialversetzungen, sofern die Spannung zurückgenommen wird, wurde ebenfalls in Simulationen mit bi-kristallinem Silber [100] sowie in Experimenten an fünffach verzwillingten Silberdrähten [89][100] registriert, wobei in den Experimenten jeweils lediglich das Verschwinden eines Kontrastes wahrgenommen wurde, bei dem nicht eindeutig bestimmt werden konnte, um um welche Art Defekt es sich handelt. Damit erbringt diese Arbeit den bisher einzigen Beleg dafür, dass es tatsächlich Stapelfehler sind, die im Zuge der Entladung verschwinden.

Für einkristalline Drähte sind zwei Fälle zu unterscheiden. Für Stapelfehler, die scheinbar nicht den gesamten Draht durchlaufen, sondern nur bis zu einem planaren Defekt auf dem konjugierten Gleitsystem, ergibt sich ein vergleichbares Szenario wie im Fall der verzwillingten Drähte, da auch hier davon auszugehen ist, dass die leadingPartialversetzung noch im Draht vorhanden ist. Beim Reißen fällt die Spannung, die die Partialversetzung gegen den planaren Defekt drückt, weg, und die Stapelfehlerenergie treibt die Partialversetzung zurück aus dem Draht, wodurch der Stapelfehler annihiliert wird (siehe Abbildung 5.7 (a)). Allerdings hätte ein solches Verhalten auch bei einer Entlastung der Drähte beobachtet werden müssen. Dies konnte aber in den TEM-Aufnahmen nicht bestätigt werden. Vermutlich handelte es sich hier um planare Defekte, bei denen die Partialversetzung auf das konjugierte Gleitsystem quergeglitten 
ist und die Partialversetzung somit nicht mehr im Draht präsent ist.

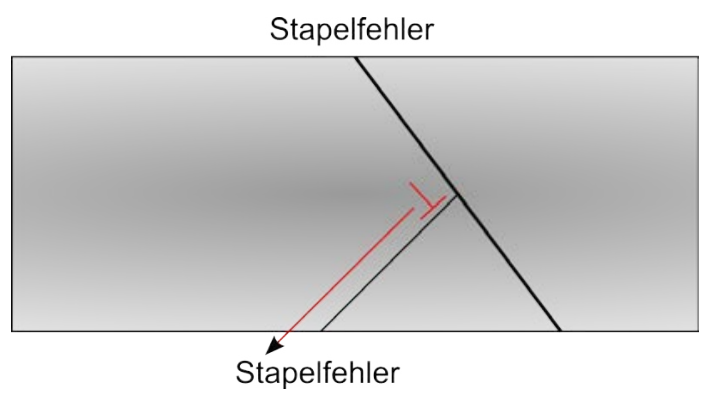

(a)

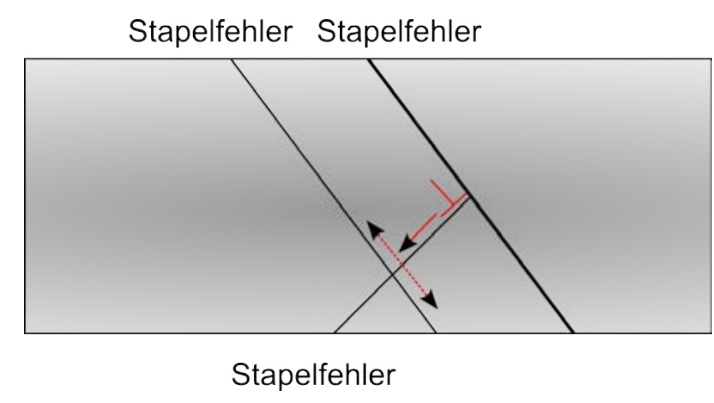

(b)

Abbildung 5.7.: Verschwinden von planaren Defekten beim Reißen

Für den Fall von Stapelfehlern, die den Draht komplett durchsetzen, scheint das bisherige Szenario nicht zutreffend zu sein, da hier davon auszugehen ist, dass die leading-Partialversetzung die Probe verlassen hat. Ebenfalls scheint die Nukleation einer trailing-Partialversetzung eher unwahrscheinlich zu sein, da diese dann alle exakt beim Reißen nukleiert worden sein müssten und gleichzeitig beim Reißen auch die Triebkraft für die Nukleation von trailing-Partialversetzungen wegfiele. Da das Verschwinden der planaren Defekte beim Reißen ebenfalls häufig in Bereichen mit vielen kreuzenden planaren Defekten beobachtet wurde, wäre ebenfalls eine stimulierte Emission von Partialversetzungen ein mögliches Szenario, analog zu der stimulierten Emission von trailing-Partialversetzung während der Verformung. So könnte eine noch im Draht befindliche Partialversetzung beim Zurücklaufen auf einen planaren Defekt des konjugierten Gleitsystems treffen und dabei die stimulierte Emission von weiteren Partialversetzungen verursachen und damit das Verschwinden von kompletten planaren Defekten hervorrufen (siehe Abbildung 5.7 (b)). 



\section{Zusammenfassung}

In der vorliegenden Arbeit wurde erfolgreich die Nukleation von Versetzungen in einkristallinen und verzwillingten Gold-Nanodrähten in umfangreichen Zugversuchen sowohl im Rasterelektronenmikroskop als auch mittels Transmissionselektronenmikroskop untersucht. Die wichtigsten Erkenntnisse der Arbeit lassen sich wie folgt zusammenfassen:

- Das elastische Verhalten von Gold-Nanodrähten entspricht dem erwarteten linearen Verlauf bei kleinen Dehnungen und dem nicht-linearen Verlauf bei höheren Dehnungen. Das ermittelte E-Modul liegt im Mittelwert mit 76GPa nahe an dem theoretischen Wert. Gleichzeitig konnte kein Größeneffekt auf das elastische Verhalten in dem untersuchten Größenbereich festgestellt werden in Übereinstimmung mit der Theorie. Sowohl die auf äußerste Sorgfalt bedachte und vorsichtige Herangehensweise bei den Versuchen als auch die gute Übereinstimmung mit dem theoretischen Wert lässt vermuten, dass die größere Streuung sowie etwaige Größeneffekte für die in der Literatur vorgestellten Daten auf Unzulänglichkeiten bei den dort vorgenommenen Messungen beruhen.

- Der Beginn der plastischen Verformung erfolgt sowohl in einkristallinen als auch in Drähten mit longitudinalem Zwilling durch die Nukleation von leadingPartialversetzungen an ausgezeichneten Punkten entlang des Drahtes, die während der Verformung nach und nach 'verbraucht' werden. Die ausgezeichneten Punkte ließen sich in den TEM-Aufnahmen deutlich nachweisen. Über Charakter, Ursprung und Struktur dieser Punkte und weshalb bei diesen Punkten die Nukleation bevorzugt abläuft, konnte in dieser Arbeit jedoch keine Aussage getroffen werden und muss weiteren Untersuchungen vorbehalten werden.

- Die Spannungen zu Beginn der plastischen Verformung liegen deutlich oberhalb von 1GPa, so dass Nanodrähte den Trend bestätigen, dass kleine Proben hohe Festigkeiten aufweisen. Die Werte für die Fließspannung sind sowohl unabhän- 
gig von der charakteristischen Größe der Drähte als auch von der Präsenz von longitudinalen Zwillingsgrenzen, was konsistent zu dem Modell einer Oberflächennukleation ist. Zudem lassen sich die gemessenen Spannungen mit der Nukleationsspannung für eine leading-Partialversetzung an der Oberfläche bzw. an einer Ecke aus dem Modell der klassischen Nukleationstheorie in Einklang bringen.

- Im Gegensatz zu bisherigen Arbeiten sowie MD-Simulationen konnte häufig die Nukleation von trailing-Partialversetzungen beobachtet werden, die nach dem Modell der klassischen Nukleationstheorie erst bei deutlich höheren Spannungen zu erwarten sind. Somit eröffnet sich die Frage, ob das Modell der klassischen Keimbildung die Versetzungsnukleation in Nanodrähten korrekt darzustellen vermag.

- Bei Drähten, in die vorher Versetzungen eingebracht worden sind, konnte kein abweichendes Verformungsverhalten festgestellt werden. Dies bestätigt die Vermutung, dass Versetzungen in nanoskaligen Proben mit einer geringen Versetzungdichte diese einfach verlassen können und die anschließende Verformung ebenfalls nukleationskontrolliert erfolgt.

- Proben mit longitudinaler Zwillingsgrenze zeigen ein verändertes Verformunsgsbild und weisen die Speicherung von Versetzungen auf. Im Gegensatz zu einkristallinen Drähten kann eine leading-Partialversetzung von der Zwillingsgrenze aufgehalten werden, was eine weitgehende Übereinstimmung mit MD-Simulationen und experimentellen Studien in der Literatur aufweist. Im Fall der Nukleation einer trailing-Partialversetzung kann diese mit der aufgestauten leadingPartialversetzung zu einer vollen Versetzung rekombinieren und dadurch die Zwillingsgrenze überwinden. Hierdurch bleibt eine immobile Versetzung im Draht zurück. Bei Wegnahme der Spannung, sei es durch Entladen oder Versagen des Drahtes, gleiten die verbleibenden leading-Partialversetzung durch die abstoßende Wirkung der Zwillingsgrenze und aufgrund der Stapelfehlerenergie wieder aus dem Draht heraus, wodurch es zu einer Annihilierung der Stapelfehler kommt und lediglich Versetzungen in dem Draht gespeichert werden.

- Das Versagen der Drähte erfolgt innerhalb eines größeren Zwillings, vermutlich durch volle Versetzungen, was mit der Abhängigkeit einer Versetzungsnukleation von der Orientierung in Einklang zu bringen ist, die im verzwillingten Bereich unterschiedlich zum undeformierten Bereich der Probe ist. Die plastische 
Dehnung bis zum Versagen unterliegt einigen Schwankungen. Es konnte kein Effekt hinsichtlich des Durchmessers oder eines weiteren Parameters festgestellt werden mit Ausnahme des Einflusses der Aktuation bzw. Dehnrate, was einen neuen Aspekt im Hinblick auf die plastische Dehnung darstellt, wofür eine exakte Begründung bisher jedoch nicht bekannt ist.

Es hat sich somit herausgestellt, das Gold-Nanodrähte ein gutes Modellsystem für die Untersuchung einer nukleationskontrollierten Verformung sowie der Überprüfung von vorhandenen Modellen darstellen. So konnten Merkmale und Eigenschaften des beobachteten Verformungsverhaltens und der gemessenen Spannungs-Dehnungs-Kurven festgestellt werden, die sich in weitgehender Übereinstimmung mit dem klassischen Modell einer Keimbildung befinden. Allerdings konnten auch Details und Aspekte aufgedeckt werden, die nicht oder nur schwierig in Einklang zu bringen sind mit dem klassischen Modell und offen bleiben für weitere Untersuchungen. 



\section{A. Einfluss der Platinschicht}

Bei der Beschreibung der verwendeten Drähte (siehe Kapitel 4.1) wurde darauf hingewiesen, dass sie nach dem Transferprozess von einer auf Platin und Kohlenstoff basierenden Kontaminationsschicht umhüllt sind. Diese entsteht zum einen durch die Wechselwirkung des Elektronenstrahls mit organischen Molekülen in den jeweiligen Vakuumkammern und zum anderen durch den Platin-Gasprecursor für die Abscheidungen der Platin-Befestigungen. Letzteres scheint hierbei der dominante Faktor zu sein, da die Kontaminationsschicht in der Mitte des Drahtes lediglich eine Stärke im Bereich von 2nm bis 5nm aufweist und zu den Platinbefestigungen hin auf eine Stärke zwischen 10nm und 20nm anwächst.

Bei der Auswertung der Spannungswerte wurde der Einfluss der Kontaminationsschicht vernachlässigt, da davon ausgegangen wurde, dass sie nur einen zu vernachlässigenden Teil der Kraft trägt. Die Steifigkeit von verschiedenen ElektronenstrahlAbscheidungen aus Gasprecursorn liegt im Bereich zwischen 10GPa und 100GPa [106][107]. Basierend auf diesen Messungen für die Steifigkeit wird ein Anteil zwischen $3 \%$ und $36 \%$ von einer $5 \mathrm{~nm}$ dicken Kontaminationsschicht in einem Golddraht mit 75nm Durchmesser getragen [12], so dass ein Einfluss der Kontaminationsschicht grundsätzlich auszuschließen ist. Allerdings konnten Chen et al. [41] in ihren Studien keine signifikanten Unterschiede in den Spannungsmessungen durch eine Zunahme der Dicke der Kontaminationsschicht um $\approx 100 \%$ entweder durch gezielte Abscheidung des Platin-Precursors über die gesamte Drahtlänge oder durch Langzeit-Abbildung mit dem Elektronenstrahl feststellen. Auch in weiteren Arbeiten in der Literatur [50][108], in denen ein Platin-Precursor für die Befestigungen bei mechanischen Tests Verwendung findet, wurde von keinem signifikanten Einfluss der Platinschicht auf Spannungsmessungen berichtet. Es ist also davon auszugehen, dass auch in dieser Arbeit die Kontaminationsschicht einen zu vernachlässigen Effekt auf die Spannungsmessung hat.

Um den Einfluss der Platinschicht auf den Verformungsmechanismus genauer zu 


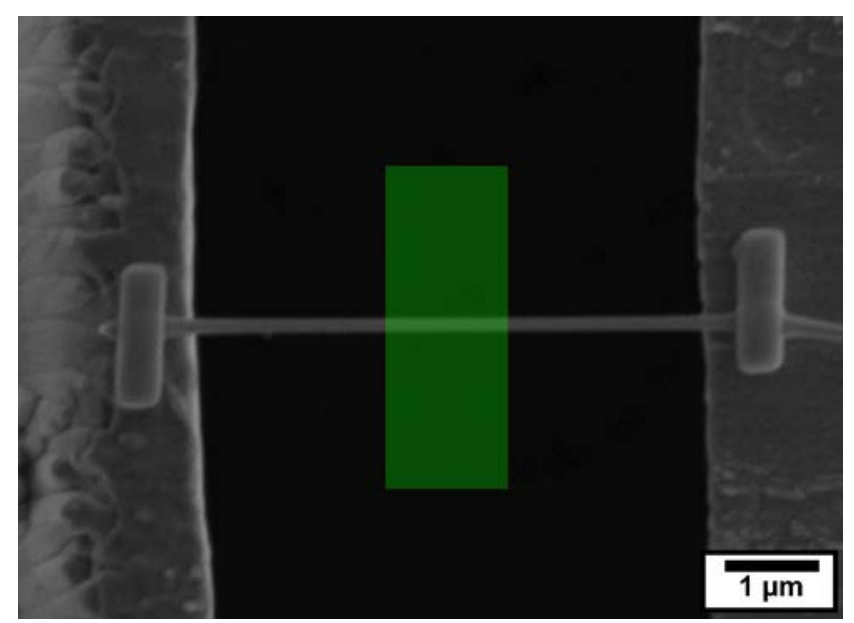

(a)

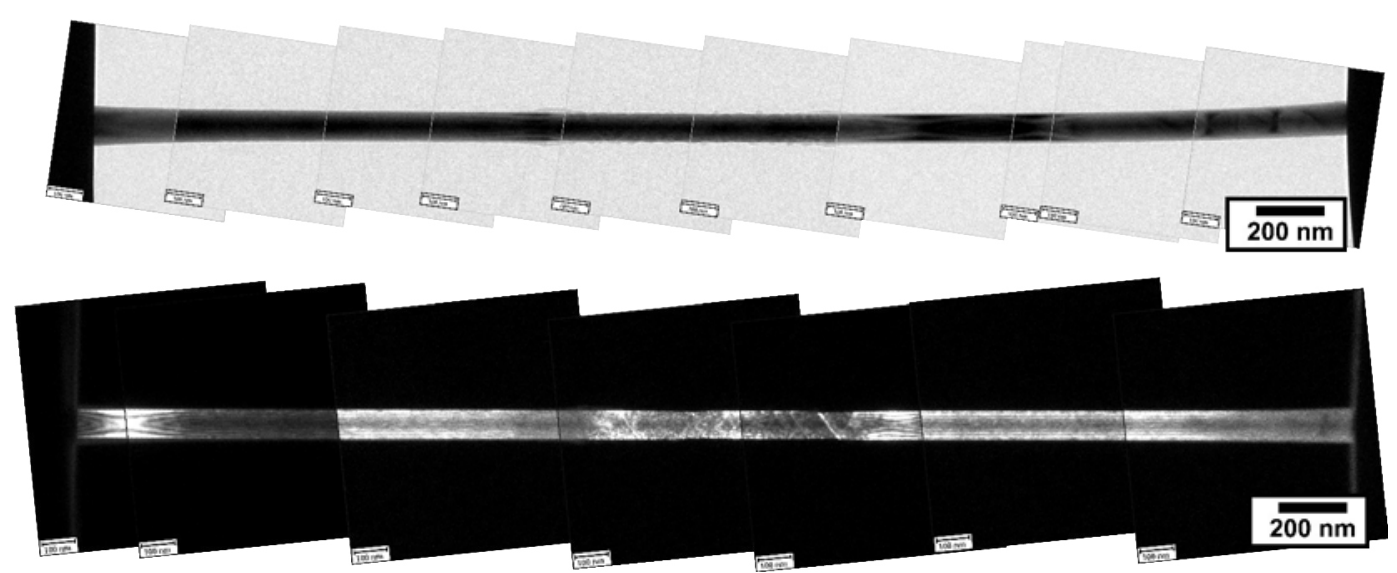

(b)

Abbildung A.1.: Einfluss der Platinschicht auf das Verformungsverhalten: Das obere Bild zeigt den Draht auf dem CuBe-Grid und den mit grün gekennzeichneten Rasterbereich. Das mittlere Bild stellt eine Hellfeldaufnahme des Drahtes nach dem Einbau ins TEM dar, in dessen mittlerem Bereich die Reduktion der Platin-Kohlenstoff-Schicht zu erkennen ist. Das untere Bild zeigt den Draht während der Verformung. Aktivität, d.h. das Bilden und Verschwinden planarer Defekte ist nur in dem mittleren Bereich festzustellen. 
untersuchen, wurde im Vorfeld eines Tests versucht, die Platinschicht zu entfernen. Dazu wurde ein Draht wie gewohnt auf ein CuBe-Grid transferiert und anschließend im REM in einer Wasserdampf-Atmosphäre mit einem definierten Rasterbereich in der Mitte des Drahtes für 10min abgebildet, um auf diese Weise eine Art Plasmacleaning durchzuführen (siehe Abbildung A.1).

Abbildung A.1 zeigt den Draht nach dem Einbau in den Zughalter in einer Hellfeldaufnahme im TEM. In dem Rasterbereich ist eindeutig eine Veränderung zu erkennen. Zum einen ist die Platin-Kohlenstoff-Schicht nahezu komplett verschwunden. An den beiden Kanten des Bereichs scheint sich die Schicht dagegen leicht verbreitert zu haben. Zum anderen erkennt man innerhalb des Bereichs einen speckigen Kontrast. Hierbei handelt es sich vermutlich um Platinkristallite, die nach dem Ablösen des Kohlenstoffs auf der Oberfläche des Drahtes zurückbleiben.

Im Zuge der Verformung lässt sich ausschließlich die Nukleation in dem Bereich beobachten, der keine äußere Schicht mehr aufweist (siehe Abbildung A.1). Ein Wechsel im Verformungsmechanismus im Vergleich zu den Versuchen mit Standard-Drähten konnte nicht festgestellt werden. Es konnte auch für diesen derart präparierten Draht das Entstehen, Verbreitern und auch das Verschwinden von planaren Defekten beobachtet werden, so dass auch in diesem Fall von der Nukleation von leading-, twinningund trailing-Partialversetzungen ausgegangen werden kann.

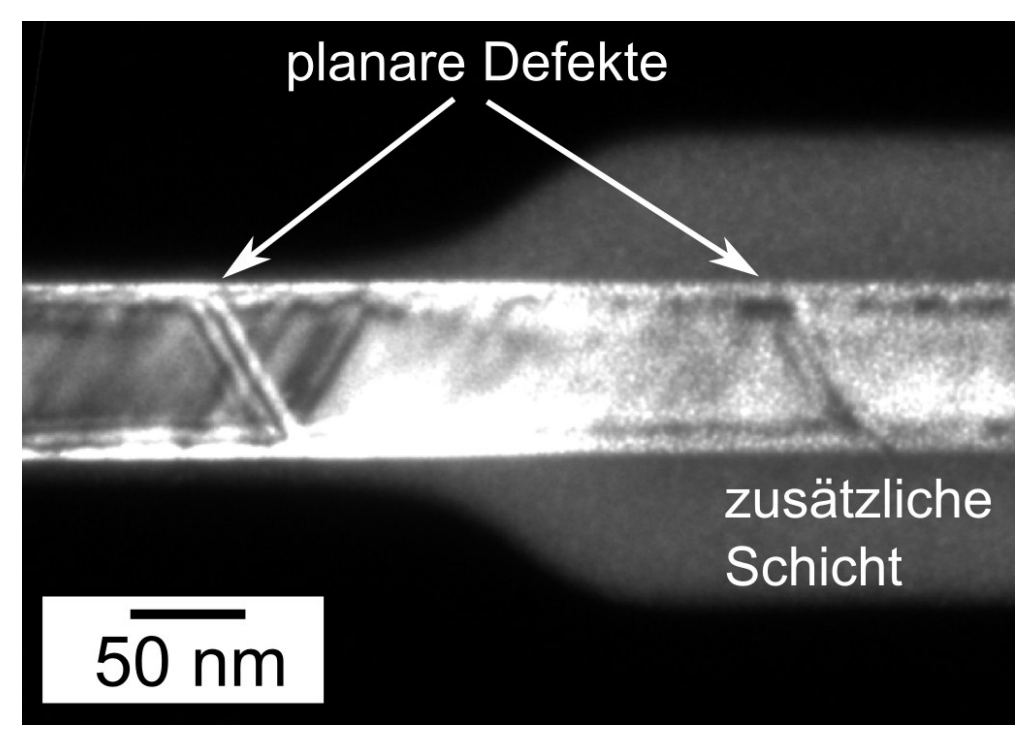

Abbildung A.2.: Einfluss einer zusätzlichen Kontaminationsschicht: Planare Defekte lassen sich sowohl in dem Bereich des Drahtes ohne (links) als auch mit (rechts) zusätzlicher Kontaminationsschicht erkennen. 
In einem weiteren Versuch wurde statt des Plasmacleanings eine Verbreiterung der Kontaminationsschicht um einen Faktor $\approx 10$ in der Mitte des Drahtes herbeigeführt. Abbildung A.2 zeigt eine Dunkelfeldaufnahme vom Übergang der Bereiche ohne (links) und mit (rechts) zusätzlicher Kontaminationsschicht. Im Zuge der Verformung lässt sich in beiden Bereichen die Entstehung von planaren Defekten beobachten.

Es lässt sich also abschließend festhalten, dass eine Kontaminationsschicht keinen Einfluss auf den Verformungsmechanismus der untersuchten Nanodrähte hat. 


\section{B. Einfluss des Elektronenstrahls}

Um den Einfluss einer sehr hohen Dosis von Elektronen auf das Verformungsverhalten zu untersuchen, wurde bei einem Zugversuch im ETEM der Elektronenstrahl sehr stark zusammengezogen und nur ein bestimmter Teil des Drahtes mit diesem fokussierten Strahl abgelichtet. Dieser Draht wies bereits vor dem Zugversuch einige planare Defekte auf, was auf eine Zugbeanspruchung während des Transferprozesses zurückzuführen ist.
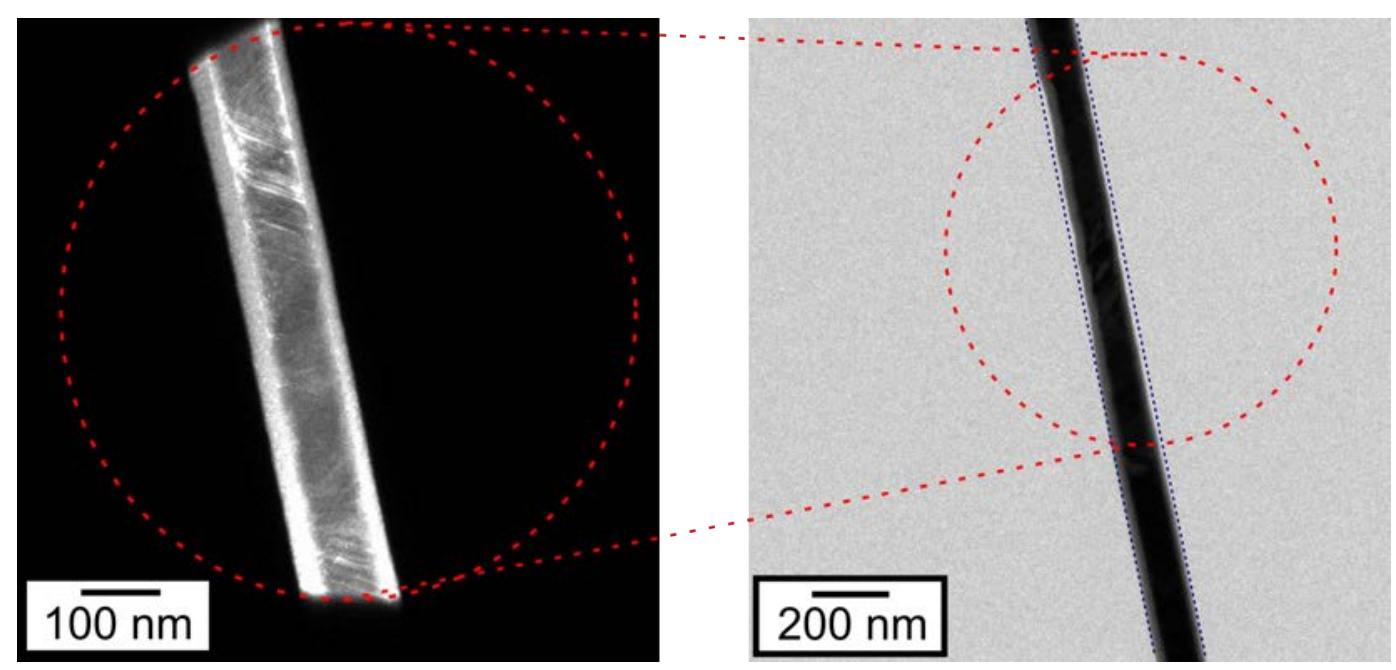

Abbildung B.1.: Einfluss des Elektronenstrahls auf das Verformungsverhalten: Das linke Bild zeigt eine Dunkelfeldaufnahme des Drahtes während der Verformung. Der rot gestrichelte Kreis markiert den Radius des zusammengezogenen Strahls. Eine Reihe von planaren Defekten sind in diesem Bereich erkennbar. Das rechte Bild zeigt eine Hellfeldaufnahme im weiteren Verlauf der Dehnung. Der rot gestrichelte Kreis markiert den Bereich des fokussierten Strahls während der Verformung. Man erkennt eine deutliche Verjüngung des Drahtes in genau diesem Bereich im Vergleich zum restlichen Draht.

Der Draht wurde nun unter normalen Abbildebedingungen bis zum ersten Erscheinen von neuen Defekten gedehnt und anschließend der Strahl stark auf einen kleinen Bereich des Drahtes fokussiert (siehe roter Kreis im linken Teil von Abbildung B.1). Durch das Bündeln des Strahls erhöhte sich die Nukleation in diesem Bereich 
schlagartig. Es bildeten sich viele neue planare Defekte, ebenso verschwanden scheinbar viele planare Defekte wieder. Durch die erhöhte Nukleation in dem bestrahlten Bereich kommt es im weiteren Verlauf der Dehnung zu einer Verjüngung in genau diesem Bereich, wie im rechten Teil von Abbildung B.1 zu sehen ist. Zur Verdeutlichung markieren die blau-gestrichelten Linien die Kanten des Drahtes in dem unbeleuchteten Teil. Die blauen gestrichelten Linien markieren die Kanten. Der Bereich des fokussierten Strahls ist wiederum durch einen roten Kreis markiert und deckt sich gut mit dem verjüngten Bereich des Drahtes.

Es lässt sich also festhalten, dass der Elektronenstrahl die Nukleation beschleunigen kann. Es kommt jedoch nicht zu einem Wechsel der beobachteten Defekte, so dass die Art der Nukleation sich nicht verändert hat. 


\section{Zugversuche an Kupfer- und Silberdrähten}

Neben Golddrähten finden sich in der Literatur eine Reihe weiterer Untersuchungen in Hinblick auf fcc-Nanodrähte, die mitunter andere Verformungsmechanismen aufweisen [41][50][83]. Im Rahmen dieser Arbeit erfolgte abgesehen von der Untersuchung von Golddrähten auch die Prüfung einiger Kupfer- und Silberdrähte, die ebenfalls aus der Kooperation mit Gunther Richter stammen und auf die selber Art und Weise hergestellt wurden [50].

Diese Proben weisen ebenfalls eine axiale $\langle 110\rangle$ Ausrichtung auf und waren entweder einkristallin oder hatten kohärente $\Sigma 3\{111\}$ Zwillingsgrenzen entlang ihrer Wachstumslänge. Im Gegensatz zu Gold-Nanodrähten ist deren Oberfläche wesentliche rauer und von einer dünnen Oxidschicht umgeben [14]. Versetzungen ließen sich vor dem Versuch nicht beobachteten. Der Transferprozess sowie der Test erfolgte analog zu den Golddrähten wie in Abschnitt 3.3 beschrieben. Insgesamt wurden 3 Kupferdrähte sowie 2 Silberdrähte einem Test im REM unterzogen und anschließend deren Drahtsegmente im TEM untersucht.

\section{C.1. Ergebnisse Kupfer}

Abbildung C.1 zeigt die Spannungs-Dehnungs-Kurven der Kupferdrähte, wobei in (a) die Kurven der einkristallinen Drähte und in (b) die Kurve eines verzwillingten Drahtes mit zwei Entladesegmenten wiedergegeben sind. Alle Drähte zeigen einen linearen Anfangsbereich bis zu einer Dehnung von circa 1\%. Die E-Module liegen zwischen 100GPa und 111GPa. Ein reversibles elastisches Verhalten bestätigte sich durch die erste Entlastung von Cu2, wobei die Entladekurve von einer Dehnung von circa 1\% und einer Spannung von circa $1 \mathrm{GPa}$ identisch zur ersten Beladungskurve zurück zum Ursprung 


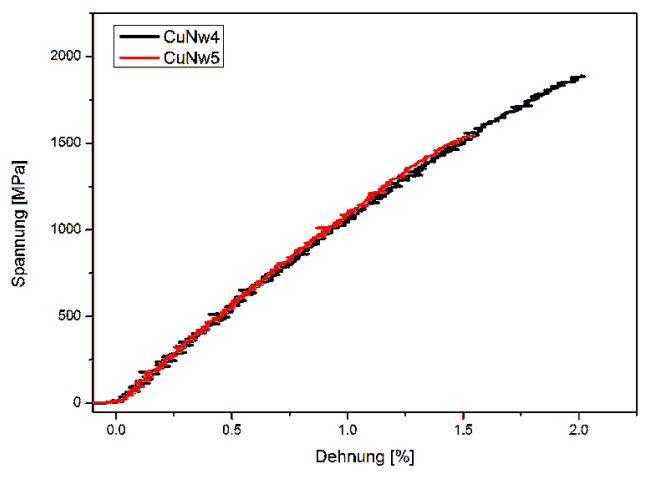

(a)

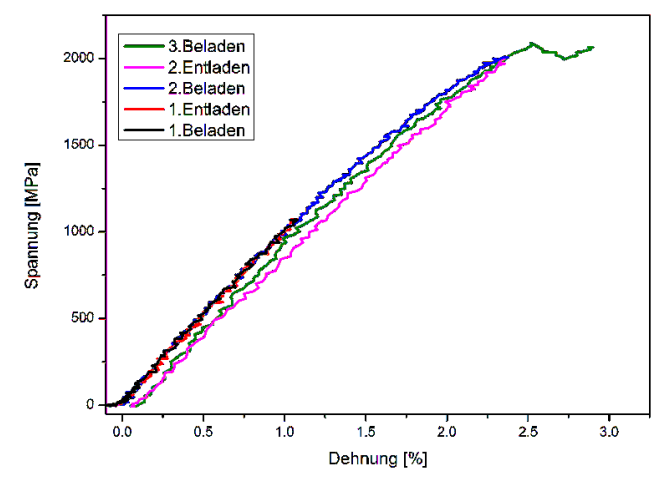

(b)

Abbildung C.1.: Spannungs-Dehnungs-Kurven Kupfer-Nanodrähte: (a) einkristalline Drähte; (b) verzwillingter Draht mit 2 Entladesegmenten

verläuft.

Ab einer Dehnung von circa 1\% nimmt die Steigung der Kurven nach und nach ab, was sowohl durch nicht-lineare Elastizität als auch den kontinuierlichen Übergang zur plastischen Verformung erklärt werden kann. Letzteres bestätigt eindeutig die zweite Entladung von $\mathrm{Cu} 2$ beginnend bei einer Dehnung von 2,3\% und einer Spannung 2GPa bestätigt, nach der eine Dehnung von circa 0,1\% im Draht verbleibt. Die Fließspannung, evaluiert bei einer Offset-Dehnung von $0,2 \%$, variiert von $1,54 \mathrm{GPa}(\mathrm{Cu} 5)$ bis auf 1,83GPa (Cu2). Die beiden einkristallinen Drähte versagen, ohne ein deutliches Abknicken der Kurve in den plastischen Bereich aufzuweisen, so dass die plastische Dehnung beim Versagen mit 0,15\% bzw. 0,25\% sehr gering ausfällt. Der verzwillingte Draht zeigt ebenfalls nur eine schwache plastische Dehnung beim Versagen von 0,8\%. Im Gegensatz zu den einkristallinen Drähten trat hier bei einer Spannung von 2,08GPa ein Abknicken der Kurven mit einem leichten Spannungsabfall auf. Die wichtigsten Größen der Spannungs-Dehnungs-Kurven sind in Tabelle C.1 zusammengefasst.

\begin{tabular}{c|c|c|c} 
& $\mathrm{Cu} 4$ & $\mathrm{Cu} 5$ & $\mathrm{Cu} 2$ (Zwilling) \\
\hline Durchmesser d & $92 \mathrm{~nm}$ & $99 \mathrm{~nm}$ & $58 \mathrm{~nm}$ \\
E-Modul E & $111 \mathrm{GPa}$ & $108 \mathrm{GPa}$ & $100 \mathrm{GPa}$ \\
Fließspannung $\sigma_{s}$ & $1,54 \mathrm{GPa}$ & $1,8 \mathrm{GPa}$ & $1,83 \mathrm{GPa}$ \\
plastische Dehnung $\epsilon_{p l}$ & $0,15 \%$ & $0,25 \%$ & $0,85 \%$ \\
\hline
\end{tabular}

Tabelle C.1.: Zusammenfassung der Messergebnisse der Kupfer-Drähte

Das E-Modul der Drähte liegt unterhalb des theoretischen Wertes von 130,5GPa für Kupfer in $\langle 110\rangle$-Richtung [58]. Die Abweichung vom theoretischen Wert fällt damit 
für die Kupfer-Drähte etwas höher aus als für die Gold-Drähte. Als wahrscheinlichste Fehlerquelle kommen hier die Messungen der Querschnittflächen in Frage. Im Gegensatz zu den Golddrähten wurden für die Kupferdrähte keine Querschnittslamellen angefertigt. Es erwies sich für die Kupferdrähte als schwierig, Querschnittbilder im FIB mit einer guten Qualität aufzunehmen. Querschnittslamellen wurden von Kupfer nicht angefertigt. Daneben weisen die Kupferdrähte nicht die gleiche Perfektion im Vergleich zu Golddrähten auf, so dass auch kleine Dickenvariationen entlang der Drähte möglich sind. Zusätzlich kommt die Oxidschicht als weitere Fehlerquelle in Betracht.

Die Werte für die Fließspannungen zeigen auch für die Kupferdrähte, dass kleine Proben eine höhere Festigkeit als ihre Bulk-Gegenstücke aufweisen. Im Vergleich mit angegeben Werten in der Literatur für Cu-Nanodrähte der gleicher Größenordnung, die von Fließspannungen zwischen 1,5GPa und 7GPa berichten [50], liegen die Werte für die Fließspannung in dieser Arbeit am unteren Ende. Für die Drähte aus der Literatur konnte jedoch partiell ein nicht unerheblicher Anteil Silizium festgestellt werden [109], was vermutlich die in einigen Fällen deutlich größeren Fließspannungen erklärt.

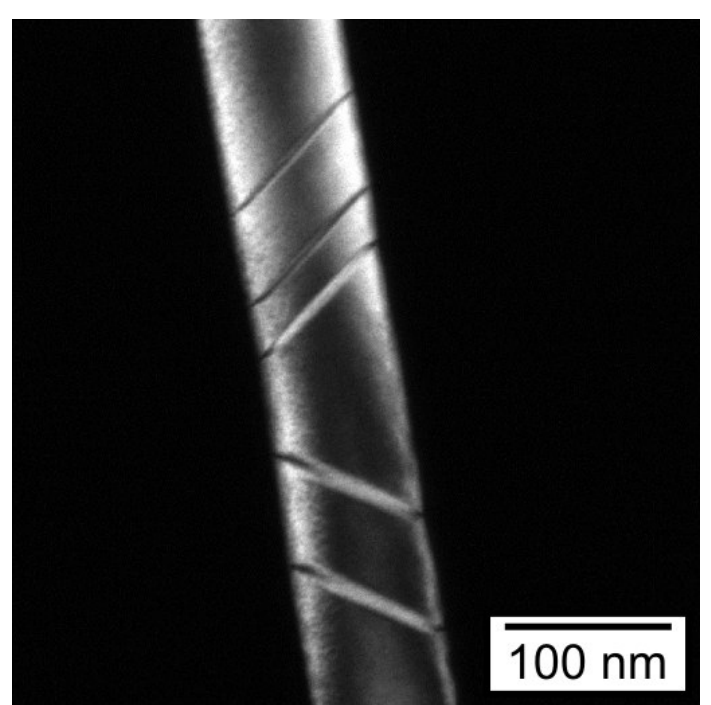

(a)

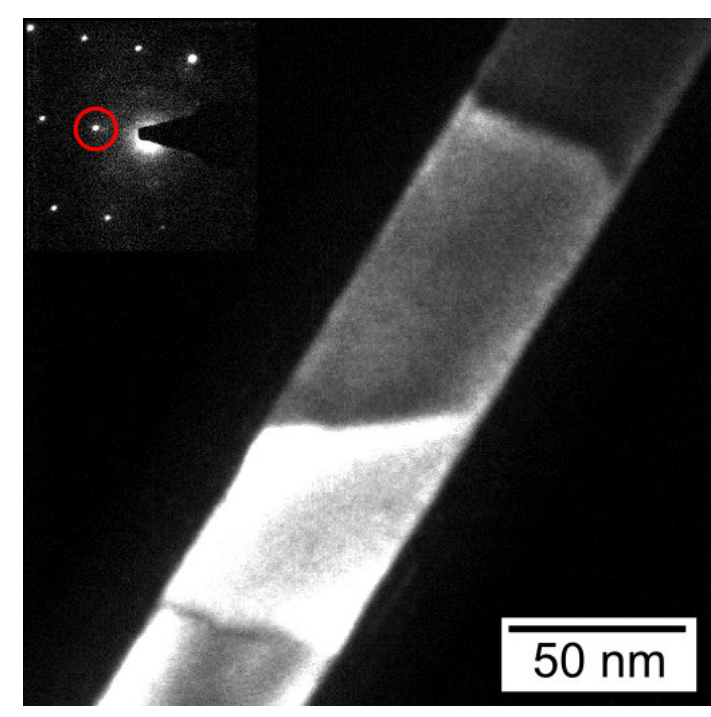

(b)

Abbildung C.2.: TEM-Dunkelfeldaufnahme von $\mathrm{Cu} 4$ (a) und $\mathrm{Cu} 2$ (b)

In den post-mortem TEM Analysen von Cu4 ließen sich entlang des Drahtes einige planare Defekte erkennen, wie Abbildung C.2 (a) zeigt. So ist auch im Fall von Kupfer von der Nukleation von leading- Partialversetzungen auszugehen. In dem verzwillingten Draht konnte keine Speicherung von planaren Defekten beobachtet werden. Stattdessen sind entlang des Drahtes vereinzelt Versetzungen zu finden wie Abbildung C.2 (b) 
verdeutlicht.

\section{C.2. Ergebnisse Silber}

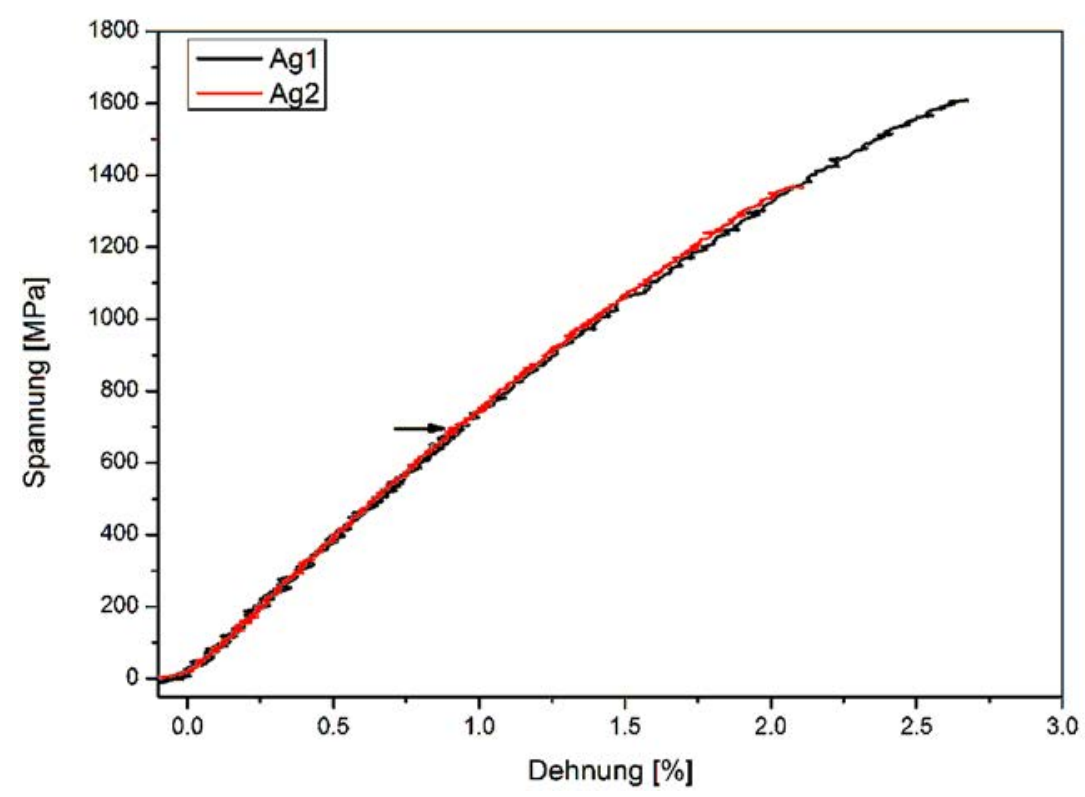

Abbildung C.3.: Spannungs-Dehnungs-Kurven Silber-Nanodrähte. Der schwarze Pfeil markiert den Beginn der Entladung von Ag1.

In Abbildung C.3 sind die Spannungs-Dehnungs-Kurven der beiden Silber-Drähte dargestellt. Beide Drähte weisen ein nahzu identisches Verhalten auf. Der Anfangsbereich zeigt einen linearen Anstieg bis zu einer Dehnung von 1\% mit einem E-Modul von $74 \mathrm{GPa}(\mathrm{Ag} 1)$ bzw. 76GPa (Ag2). Ein reversibles elastisches Verhalten wurde durch die Entlastung von Ag1 bei einer Dehnung bei 0,9\% und einer Spannung von 680MPa (schwarzer Pfeil in Abbildung C.3) bestätigt, wobei die Entladekurve und Beladekurve nahezu perfekt übereinander liegen. Ab einer Dehnung von 1\% nimmt die Steigung der Kurven langsam ab, was damit entweder eine nicht-lineare Elastizität oder den sukzessiven Beginn der Plastizität anzeigt. Die Fließspannung bei einer Offset-Dehnung von 0,2\% ergibt für beide Drähte einen Wert von 1,3GPa. Beide Drähte zeigen nur eine kleine plastische Dehnung von 0,5\% bzw. 0,3\% bevor es zum Versagen kommt. Die wichtigsten Größen der beiden Drähte sind in Tabelle C.2 zusammengefasst.

Ein Vergleich dieser Werte mit den angegebenen Werten in der Literatur ergibt, dass das Elastizitätsmodul leicht unter dem theoretischen Wert von 83,6GPa für Silber in $\langle 110\rangle$ Richtung [58] liegt. Die Fließspannungen der Silberdrähte zeigen ebenfalls 


\begin{tabular}{c|c|c} 
& Ag1 & Ag2 \\
\hline Durchmesser d & $158 \mathrm{~nm}$ & $164 \mathrm{~nm}$ \\
E-Modul E & $74 \mathrm{GPa}$ & $76 \mathrm{GPa}$ \\
Fließspannung $\sigma_{s}$ & $1,3 \mathrm{GPa}$ & $1,3 \mathrm{GPa}$ \\
plastische Dehnung $\epsilon_{p l}$ & $0,5 \%$ & $0,3 \%$ \\
\hline
\end{tabular}

Tabelle C.2.: Zusammenfassung der Messergebnisse der Silber-Drähte

eine höhere Festigkeit verglichen mit makroskopischen Proben. Die gemessenen Werte der Fließspannung fallen mit 1,3GPa im Vergleich mit anderen Messungen von Bernal et al. (1,7GPa)[89] und Filleter et al. (>2GPa)[88] kleiner aus. Gründe hierfür sind möglicherweise die Unebenheiten in der Drahtoberfläche der Drähte, die zu Spannungskonzentrationen und frühem Versagen der Drähte führen könnten. Dies wird auch dadurch bestätigt, dass beide Drähte so gut wie keine plastische Dehnung erfahren.

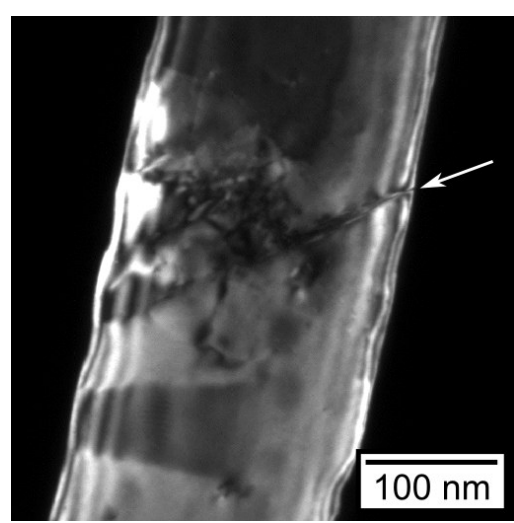

(a)

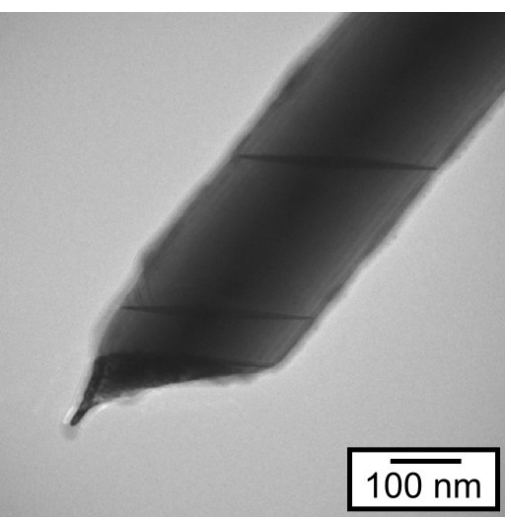

(b)

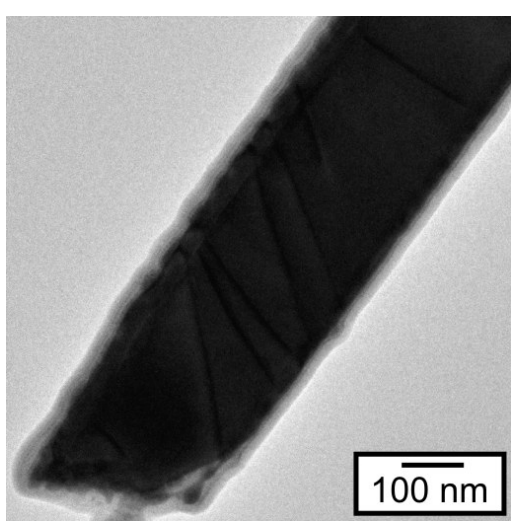

(c)

Abbildung C.4.: TEM-Dunkelfeldaufnahme von Ag1 sowie zwei Hellfeldaufnahmen von Drähten, die im Rahmen der Arbeit von Sohn [14] getestet wurden

An Hand der post-mortem TEM Aufnahmen lässt sich vermuten, dass beide Drähte entlang ihrer Achse verzwillingt sind. Abbildung C.4 (a) zeigt die Dunkelfeldaufnahme von Defekten in Draht Ag1. Hauptsächlich sind Kontraste von Versetzungen zu erkennen sowie ein planarer Defekt (markiert durch den weiß Pfeil), welcher scheinbar bis zu den Versetzungen verläuft. In Ag2 waren lediglich ein planarer Defekt sowie wenige Kontraste von Versetzungen nahe der Bruchkante auszumachen. Unter Hinzuziehung von Aufnahmen, die im Zuge der Arbeit von Sohn [14] entstanden sind, ergibt sich wieder das gleich Bild wie für Golddrähte. Abbildung C.4 (b) zeigt einen einkristallinen Silberdraht mit Stapelfehlern sowie einem Zwilling an der Bruchkante. 
Abbildung C.4 (c) zeigt einen verzwillingten Draht, in dem ausschließlich die Speicherung von Versetzungen zu beobachten waren.

\section{C.3. Fazit}

Sowohl das Verformungsverhalten als auch die Spannungs-Dehnungs-Kurven der getesteten Kupfer- und Silberdrähte ergeben ein nahezu identisches Bild zu den schwerpunktmäßig untersuchten Golddrähten. In einkristallinen Drähten konnten planare Defekte festgestellt werden, so dass auch hier die Verformung über die Nukleation von leading- und twinning-Partialversetzungen stattfindet. In den verzwillingten Drähten war größtenteils die Speicherung von Versetzungen erkennbar, so dass auch hier von einem analogen Mechanismus wie in den Gold-Drähten auszugehen ist. Auch die Spannungs-Dehnungs-Kurven zeigen einen ähnlichen Verlauf. Hohe plastische Dehnungen konnten jedoch in keinem Fall beobachtet werden, was vermutlich an der geringeren Qualität der Drähte liegt, in deren Folge es zu einer lokalen Verformung und Versagen der Drähte kommt.

Es bleibt also festzuhalten, dass das beobachtete Verhalten kein spezielles Verhalten bei Gold-Drähten darstellt, sondern offenbar ein universelles Verhalten von fcc-Metall-Nanodrähten darstellt. Dies mag daran liegen, dass sich die drei untersuchen Metallarten sehr ähneln, insbesondere was die Stapelfehlerenergie betrifft (Gold $40 \mathrm{~mJ} / \mathrm{m}^{2}$; Kupfer $40 \mathrm{~mJ} / \mathrm{m}^{2}$; Silber $\left.20 \mathrm{~mJ} / \mathrm{m}^{2}\right)$. Interessant wäre es, Metall-Drähte zu testen, deren Stapelfehlerenergie sich stark von den bisher getesteten Metallen unterschiedet, und zu untersuchen, welche Auswirkungen dies auf deren Verformungsverhalten hat. Als Untersuchungsobjekte böten sich hier Pd-Drähte $\left(169 \mathrm{~mJ} / \mathrm{m}^{2}\right)$ oder Al-Drähte $\left(166 m J / m^{2}\right)$ an. 


\section{Literaturverzeichnis}

[1] Brenner, S.S.: Tensile Strength of Whiskers. Journal of Applied Physics, 27(12):1484-1491, 1956.

[2] Brenner, S.S.: Plastic deformation of copper and silver whiskers. Journal of Applied Physics, 28(9):1023-1026, 1957.

[3] Brenner, S.S.: Growth and Properties of Whiskers. Science, 128(3324):569-575, 1958.

[4] Brenner, S.S.: Strength of gold whiskers. Journal of Applied Physics, 30:266$267,1959$.

[5] Shan, Z. W., Raja K. Mishra, S. A. Syed Asif, Oden L. Warren und ANDREW M. Minor: Mechanical annealing and source-limited deformation in submicrometre-diameter Ni crystals. Nat Mater, 7(2):115-119, Februar 2008.

[6] Lilleodden, E.T. und W.D. Nix: Microstructural length-scale effects in the nanoindentation behavior of thin gold films. Acta Materialia, 54(6):1583-1593, 2006.

[7] Sudharshan Phani, P., K. E. Johanns, E. P. George und G. M. Pharr: A simple stochastic model for yielding in specimens with limited number of dislocations. Acta Materialia, 61(7):2489-2499, April 2013.

[8] LeE, S. W. und W. D. NIX: Geometrical analysis of 3D dislocation dynamics simulations of FCC micro-pillar plasticity. Materials Science and Engineering: A, 527(7-8):1903-1910, März 2010.

[9] El-Awady, Jaafar A., Ming Wen und Nasr M. Ghoniem: The role of the weakest-link mechanism in controlling the plasticity of micropillars. Journal of the Mechanics and Physics of Solids, 57(1):32 - 50, 2009. 
[10] Zhu, Ting, Ju Li, Amit Samanta, Austin Leach und Ken Gall: Temperature and Strain-Rate Dependence of Surface Dislocation Nucleation. Phys. Rev. Lett., 100(2):025502-, Januar 2008.

[11] Roos, Burkhard: In-situ Zugversuche an einkristallinen Gold-Nanodrähten im TEM. Dissertation, Institut für Materialphysik, Universität Göttingen, 2012.

[12] KAPELLE, B: In situ Zugversuch an einkristallinen Gold-Nanodrähten im REM. Diploma Thesis, Institut für Materialphysik, Universität Göttingen, 2011.

[13] MeckenhäUser, Christian: In-situ Zugversuch an einkristallinen Metallnanodrähten im REM. Bachelor Thesis, Institut für Materialphysik, Universität Göttingen, 2011.

[14] Sohn, Florian: In-situ Zugversuch an Silbernanodrähten im REM. Bachelor Thesis, Institut für Materialphysik, Universität Göttingen, 2012.

[15] Osterthun, Norbert: Ultrasschnelle in-situ Druckversuche im REM. Bachelor Thesis, Institut für Materialphysik, Universität Göttingen, 2014.

[16] Hinth, J.P. und J. Lothe: Theory of dislocations. McGraw-Hill, 1968.

[17] Li, B.Q., M.L. Sui und S.X. MaO: Twinnability Predication for fcc Metals. Journal of Materials Sciences and Technology, 27:97-100, 2011.

[18] Park, Harold S., Ken Gall und Jonathan A. Zimmerman: Deformation of FCC nanowires by twinning and slip. Journal of the Mechanics and Physics of Solids, 54(9):1862-1881, September 2006.

[19] Weinberger, Christopher R. und Wei Cai: Plasticity of metal nanowires. Journal of Materials Chemistry, 22(8):3277-3292, 2012.

[20] Seo, Jong-Hyun, Youngdong Yoo, Na-Young Park, Sang-Won Yoon, Hyoban lee, Sol Han, Seok-Woo Lee, Tae-Yeon Seong, Seung-Cheol LeE, Kon-BAE LeE et al.: Superplastic deformation of defect-free Au nanowires via coherent twin propagation. Nano Letters, 11(8):3499-3502, 2011.

[21] Sedlmayr, Andreas, Erik Bitzek, Daniel S. Gianola, Gunther RichTER, Reiner MÖnIG und Oliver Kraft: Existence of Two Twinning- 
Mediated Plastic Deformation Modes in Au Nanowhiskers. Acta Materialia, 60(9):3985-3993, Mai 2012.

[22] Lee, Subin, Jiseong Im, Youngdong Yoo, Erik Bitzek, Daniel Kiener, Gunther Richter, Bongsoo Kim und Sang Ho Oh: Reversible cyclic deformation mechanism of gold nanowires by twinning-detwinning transition evidenced from in situ TEM. Nature Communications, 5:3033, 2014.

[23] Tadmor, E.B. und S. HAI: A Peierls criterion for the onset of deformation twinning at a crack tip. Journal of the Mechanics and Physics of Solids, 51(5):765-793, Mai 2003.

[24] Van Swygenhoven, H., P. M. Derlet und A. G. Froseth: Stacking fault energies and slip in nanocrystalline metals. Nature Materials, 3(6):399-403, Juni 2004.

[25] ViteK, V.: Intrinsic stacking faults in body-centred cubic crystals. Philosophical Magazine, 18(154):773-786, Oktober 1968.

[26] Rice, James R.: Dislocation nucleation from a crack tip: An analysis based on the Peierls concept. Journal of the Mechanics and Physics of Solids, 40(2):239-271, 1992.

[27] Weinberger, Christopher R., Andrew T. Jennings, Keonwook Kang und Julia R. GReER: Atomistic simulations and continuum modeling of dislocation nucleation and strength in gold nanowires. Journal of the Mechanics and Physics of Solids, 60(1):84-103, 2012.

[28] Hwang, Byungil, Mijeong Kang, Subin Lee, Christopher R. WeinberGer, Phillip Loya, Jun Lou, Sang Ho Oh, Bongsoo Kim und Seung Min HAN: Effect of surface energy on size-dependent deformation twinning of defectfree Au nanowires. Nanoscale, 7:15657-15664, 2015.

[29] Leach, M., M. McDowell und K. Gall: Deformation of Top-Down and Bottom-Up Silver Nanowires. Adv. Funct. Mater., 17(1):43-53, 2007.

[30] Jiang, Jin-Wu, Austin M. Leach, Ken Gall, Harold S. Park und TiMON RABCZUK: A surface stacking fault energy approach to predicting defect 
nucleation in surface-dominated nanostructures. Journal of the Mechanics and Physics of Solids, 61(9):1915 - 1934, 2013.

[31] BEcker, R. und W. DöRING: Kinetische Behandlung der Keimbildung in übersättigten Dämpfen. Annalen der Physik, 416(8):719-752, 1935.

[32] Jonsson, H., G. Mills und K. W. Jacobsen: Nudged elastic band for finding minimum energy paths of transitions. World Scientific, 1998.

[33] Hirel, P., S. Brochard, L. Pizzagalli und P. Beauchamp: Effects of temperature and surface step on the incipient plasticity in strained aluminium studied by atomistic simulations. Scripta Materialia, 57(12):1141 - 1144, 2007.

[34] Hirel, P., J. Godet, S. Brochard, L. Pizzagalli und P. Beauchamp: Determination of activation parameters for dislocation formation from a surface in fcc metals by atomistic simulations. Physical Review B, 78(6):064109, August 2008 .

[35] Jennings, Andrew T, Christopher R Weinberger, Seok-Woo Lee, Zachary H Aitken, Lucas Meza und Julia R Greer: Modeling dislocation nucleation strengths in pristine metallic nanowires under experimental conditions. Acta Materialia, 61(6):2244-2259, April 2013.

[36] Bobylev, SV und AG Sheinerman: CRITICAL STRESS FOR DISLOCATION FORMATION IN DEFORMED TWINNED NANOWIRES. Rev. Adv. Mater. Sci, 43:31-37, 2015.

[37] Beltz, G. E. und L. B. Freund: On the Nucleation of Dislocations at a Crystal Surface. physica status solidi (b), 180(2):303-313, 1993.

[38] Kapelle, B, L. Pizzagalli und C. Volkert: Private communication. 2015.

[39] NGan, A.H.W, L Zuo und P.C Wo: Size dependence and stochastic nature of yield strength of micron-sized crystals: a case study on Ni3Al. Proceedings of the Royal Society of London A: Mathematical, Physical and Engineering Sciences, 462(2070):1661-1681, 2006.

[40] Hyde, B., Horacio, D. Espinosa und Diana Farkas: An Atomistic Investigation of Elastic and Plastic Properties of Au Nanowires. Journal of Materials Research, Seiten 62-66, 2005. 
[41] Chen, Lisa Y, Mo-Rigen He, Jungho Shin, Gunther Richter und DaNIEL S GIANOLA: Measuring surface dislocation nucleation in defect-scarce nanostructures. Nature materials, 14:707-713, 2015.

[42] Aubry, Sylvie, Keonwook Kang, Seunghwa Ryu und Wei Cai: Energy barrier for homogeneous dislocation nucleation: Comparing atomistic and continuum models. Scripta Materialia, 64(11):1043-1046, Juni 2011.

[43] Byun, T. S.: On the stress dependence of partial dislocation separation and deformation microstructure in austenitic stainless steels. Acta Materialia, 51(11):3063-3071, Juni 2003.

[44] Yamakov, V., D. Wolf, S. R. Phillpot, A. K. Mukherjee und H. GleiTER: Deformation-mechanism map for nanocrystalline metals by moleculardynamics simulation. Nature Materials, 3(1):43-47, Januar 2004.

[45] Swygenhoven, H. Van, P.M. Derlet und A.G. Froeseth: Nucleation and propagation of dislocations in nanocrystalline fcc metals. Acta Materialia, 54(7):1975-1983, 2006.

[46] Chen, Mingwei, En Ma, Kevin J. Hemker, Hongwei Sheng, Yinmin Wang und Xuemei Cheng: Deformation Twinning in Nanocrystalline Aluminum. Science, 300(5623):1275-1277, Mai 2003.

[47] Oh, S.H., M. Legros, D. Kiener, P. Gruber und G. Dehm: In situ TeM straining of single crystal Au films on polyimide: Change of deformation mechanisms at the nanoscale. Acta Materialia, 55(16):5558-5571, September 2007.

[48] Volkert, C.A. und A.M. Minor: Focused Ion Beam Microscopy and Micromachining. MRS Bulletin, 32:389-399, Mai 2007.

[49] Sun, Yu und Bradley J. Nelson: MEMS capacitive force sensors for cellular and flight biomechanics. Biomedical Materials, 2(1):S16, 2007.

[50] Richter, G., K. Hillerich, D. S. Gianola, R. Monig, O. Kraft und C. A. Volkert: Ultrahigh Strength Single Crystalline Nanowhiskers Grown by Physical Vapor Deposition. Nano Letters, 9(8):3048-3052, August 2009.

[51] Richter, Gunther: Fabrication of freestanding gold nanotubes. Scripta Materialia, 63(9):933 - 936, 2010. 
[52] Schamel, Matthias, Carola Schopf, Dominic Linsler, Sabine T HaAg, Lisa Hofacker, Christian Kappel, Horst P Strunk und Gunther RICHTER: The filamentary growth of metals. International Journal of Materials Research, 102(7):828-836, 2011.

[53] Eberl, C., D. Gianola und K. Hemker: Mechanical Characterization of Coatings Using Microbeam Bending and Digital Image Correlation Techniques. Experimental Mechanics, 50(1):85-97, Januar 2010.

[54] Burnham, N A, X Chen, C S Hodges, G A Matei, E J Thoreson, C J Roberts, M C Davies und S J B Tendler: Comparison of calibration methods for atomic-force microscopy cantilevers. Nanotechnology, 14(1):1, 2003.

[55] Technologies, Agilent: Agilent Nano Indenter G200 Data Sheet, sep 2009.

[56] Erichsen, Torben: In-situ Zugversuche an Goldnanodrähten in einem hochauflösenden Environmental Transmission Electron Microscope. Diplomarbeit, Institut für Materialphysik, Universität Göttingen, 2013.

[57] Williams, D B. und C. B. Carter: Transmission Electron Microscopy. Plenum Press, New York, 1996.

[58] Zhang, Jian-Min, Yan Zhang, Ke-Wei Xu und Vincent Ji: Young s modulus surface and Poisson s ratio curve for cubic metals. Journal of Physics and Chemistry of Solids, 68(4):503-510, April 2007.

[59] Jennett, N. M., R. Ghisleni und J. Michler: Enhanced yield strength of materials: The thinness effect. Applied Physics Letters, 95(12):123102, 2009.

[60] Sedlmayr, Andreas: Experimental Investigations of Deformation Pathways in Nanowires. KIT Scientific Publishing, 2014.

[61] Gall, K., J. Diao und M.L. Dunn: The Strength of Gold Nanowires. Nano Letters, 4(12):2431-2436, 2004.

[62] Chen, Lisa Y., Gunther Richter, John P. Sullivan und Daniel S. GiaNolA: Lattice Anharmonicity in Defect-Free Pd Nanowhiskers. Physical Review Letters, 109(12):125503-, September 2012. 
[63] Kobler, Aaron, Thorsten Beuth, Tobias Klöffel, Robby Prang, Markus Moosmann, Torsten Scherer, Stefan Walheim, Horst Hahn, Christian Kübel, Bernd Meyer, Thomas Schimmel und Erik Bitzek: Nanotwinned silver nanowires: Structure and mechanical properties. Acta Materialia, 92:299-308, 2015.

[64] Murphy, Kathryn F, Lisa Y Chen und Daniel S Gianola: Effect of organometallic clamp properties on the apparent diversity of tensile response of nanowires. Nanotechnology, 24(23):235704, 2013.

[65] Li, X.D., X.N. Wang, W.C. Chang, Y.J. ChaO und M. Chang: Effect of tensile offset angles on micro/nanoscale tensile testing. REVIEW OF SCIENTIFIC INSTRUMENTS, 76(3), MAR 2005.

[66] Miller, Ronald E. und Vijay B. Shenoy: Size-dependent elastic properties of nanosized structural elements. Nanotechnology, 11(3):139, 2000.

[67] Dingreville, Remi, Jianmin Qu und Mohammed Cherkaoui: Surface free energy and its effect on the elastic behavior of nano-sized particles, wires and films. Journal of the Mechanics and Physics of Solids, 53(8):1827 - 1854, 2005.

[68] Liang, Haiyi, Moneesh Upmanyu und Hanchen Huang: Size dependent elasticity of nanowires: Nonlinear effects. Phys. Rev. B, 71(24):241403-, Juni 2005.

[69] Zhu, Yong, Qingquan Qin, Feng Xu, Fengru Fan, Yong Ding, Tim Zhang, Benjamin J. Wiley und Zhong Lin Wang: Size effects on elasticity, yielding, and fracture of silver nanowires: In situ experiments. Physical Review B, 85(4):045443, Januar 2012.

[70] Zheng, He, Ajing Cao, Christopher R. Weinberger, Jian Yu Huang, Kui Du, Jianbo Wang, Yanyun Ma, Younan Xia und Scott X. MaO: Discrete plasticity in sub $10 \mathrm{~nm}$ sized gold crystals. Nat Commun, 1:144-, Dezember 2010.

[71] Lu, Yang, Jun Song, Jian Yu Huang und Jun Lou: Fracture of Sub 20nm Ultrathin Gold Nanowires. Advanced Functional Materials, 21(20):3982-3989, 2011. 
[72] Seo, Jong-Hyun, Harold S. Park, Youngdong Yoo, Tae-Yeon Seong, Ju Li, Jae-Pyoung Ahn, Bongsoo Kim und In-Suk Choi: Origin of Size Dependency in Coherent-Twin-Propagation-Mediated Tensile Deformation of Noble Metal Nanowires. Nano Lett., 13(11):5112-5116, November 2013.

[73] Volkert, C. A. und E. T. Lilleodden: Size effects in the deformation of sub-micron Au columns. Philosophical Magazine, 86(33):5567-5579, 2006.

[74] Dietiker, M., S. Buzzi, G. Pigozzi, J.F. Löffler und R. Spolenak: Deformation behavior of gold nano-pillars prepared by nanoimprinting and focused ion-beam milling. Acta Materialia, 59(5):2180-2192, März 2011.

[75] Greer, Julia R. und William D. Nix: Nanoscale gold pillars strengthened through dislocation starvation. Physical Review B, 73(24):245410, 2006.

[76] Greer, Julia R., Warren C. Oliver und William D. Nix: Size dependence of mechanical properties of gold at the micron scale in the absence of strain gradients. Acta Materialia, 53(6):1821-1830, April 2005.

[77] Uchic, Michael D., Dennis M. Dimiduk, Jeffrey N. Florando und William D. Nix: Sample Dimensions Influence Strength and Crystal Plasticity. Science, 305(5686):986-989, August 2004.

[78] Uchic, Michael D., Paul A. Shade und Dennis M. Dimiduk: Plasticity of Micrometer-Scale Single Crystals in Compression. Annual Review of Materials Research, 39(1):361-386, Juli 2009.

[79] Bei, Hongbin, Sanghoon Shim, George Mathews Pharr und Easo P GEORGE: Effects of pre-strain on the compressive stress-strain response of Moalloy single-crystal micropillars. Acta Materialia, 56(17):4762-4770, 2008.

[80] Phani, P. Sudharshan, K.E. Johanns, G. Duscher, A. Gali, E.P. GeORGE und G.M. PHARR: Scanning transmission electron microscope observations of defects in as-grown and pre-strained Mo alloy fibers. Acta Materialia, 59(5):2172-2179, 2011.

[81] Johanns, Kurt E., Andreas Sedlmayr, P. Sudharshan Phani, Reiner Mönig, Oliver Kraft, Easo P. George und George M. Pharr: In-situ tensile testing of single-crystal molybdenum-alloy fibers with various dislocati- 
on densities in a scanning electron microscope. Journal of Materials Research, 27(03):508-520, 2012.

[82] Chisholm, C., H. Bei, M.B. Lowry, J. Oh, S.A. Syed Asif, O.L. WarRen, Z.W. Shan, E.P. George und A.M. Minor: Dislocation starvation and exhaustion hardening in Mo alloy nanofibers. Acta Materialia, 60(5):2258-2264, 2012.

[83] Mompiou, Frederic, Marc Legros, Andreas Sedlmayr, Daniel S. Gianola, Daniel Caillard und Oliver Kraft: Source-based strengthening of sub-micrometer Al fibers. Acta Materialia, 60(3):977-983, 2012.

[84] Weinberger, Christopher R. und Wei Cai: Surface-controlled dislocation multiplication in metal micropillars. Proceedings of the National Academy of Sciences, 105(38):14304-14307, September 2008.

[85] LI, C. und G. Xu: Critical conditions for dislocation nucleation at surface steps. Philosophical Magazine, 86(20):2957-2970, Juli 2006.

[86] Brochard, S., P. Hirel, L. Pizzagalli und J. Godet: Elastic limit for surface step dislocation nucleation in face-centered cubic metals: Temperature and step height dependence. Acta Materialia, 58(12):4182-4190, Juli 2010.

[87] Bernstein, N. und E. B. TADMor: Tight-binding calculations of stacking energies and twinnability in fcc metals. Physical Review B, 69(9):094116, März 2004.

[88] Filleter, Tobin, Seunghwa Ryu, Keonwook Kang, Jie Yin, Rodrigo A. Bernal, Kwonnam Sohn, Shuyou Li, Jiaxing Huang, Wei Cai und Horacio D. Espinosa: Nucleation-Controlled Distributed Plasticity in Pentatwinned Silver Nanowires. Small, 8(19):2986-2993, Oktober 2012.

[89] Bernal, Rodrigo A., Amin Aghaei, Sanguun Lee, Seunghwa Ryu, Kwonnam Sohn, Jiaxing Huang, Wei Cai und Horacio Espinosa: Intrinsic Bauschinger Effect and Recoverable Plasticity in Pentatwinned Silver Nanowires Tested in Tension. Nano Letters, 15(1):139-146, 2015. PMID: 25279701.

[90] Lee, Sangryun und Seunghwa Ryu: Molecular Dynamics Study on the Distributed Plasticity of Penta-twinned Silver Nanowires. Frontiers in Materials, 2(56), 2015. 
[91] Zheng, He, Jiangwei Wang, Jian Yu Huang, Jianbo Wang und ScOtT X. MAO: Void-assisted plasticity in Ag nanowires with a single twin structure. Nanoscale, 6:9574-9578, 2014.

[92] Sainath, G. und B.K. Choudhary: Molecular dynamics simulation of twin boundary effect on deformation of $\mathrm{Cu}$ nanopillars. Physics Letters A, 379(34â€"35):1902 - 1905, 2015.

[93] Gilman, J. J.: Dislocation Mobility in Crystals. Journal of Applied Physics, 36:3195, 1965.

[94] Rose, Joseph L.: Ultrasonic Waves in Solid Media. Cambridge University Press, 2004.

[95] HaAsen, P.: Physikalische Metallkunde. Springer Verlag, 1992.

[96] Jin, Z.-H., P. Gumbsch, E. Ma, K. Albe, K. Lu, H. Hahn und H. Gleiter: The interaction mechanism of screw dislocations with coherent twin boundaries in different face-centred cubic metals. Scripta Materialia, 54(6):1163-1168, März 2006.

[97] Jin, Z.-H., P. Gumbsch, K. Albe, E. Mae, K. Luf, H. Gleiter und H. HAHN: Interactions between non-screw lattice dislocations and coherent twin boundaries in face-centered cubic metals. Acta Materialia, 56:1126-1135, 2008.

[98] Wu, Z.X., Y.W. Zhang und D.J. Srolovitz: Dislocation-twin interaction mechanisms for ultrahigh strength and ductility in nanotwinned metals. Acta Materialia, 57(15):4508-4518, 2009.

[99] Zhu, Y.T., X.L. Wu, X.Z. Liao, J. Narayan, L.J. Kecskés und S.N. Mathaudhu: Dislocation-twin interactions in nanocrystalline fcc metals. Acta Materialia, 59:812-821, 2011.

[100] Qin, Qingquan, Sheng Yin, Guangming Cheng, Xiaoyan Li, Tzu-Hsuan Chang, Gunther Richter, Yong Zhu und Huajian GaO: Recoverable plasticity in penta-twinned metallic nanowires governed by dislocation nucleation and retraction. Nat Commun, 6:-, Januar 2015.

[101] AshBeE, K.H.G: Intersecting stacking faults in face-centered cubic lattices. Acta Metallurgica, 15(7):1129 - 1131, 1967. 
[102] Rajan, Krishna und JohnB. Vander SAnde: Room temperature strengthening mechanisms in a Co-Cr-Mo-C alloy. Journal of Materials Science, 17(3):769-778, 1982.

[103] RAJAn, KRISHNA: Stacking fault strengthening in low stacking fault energy alloys. Scripta Metallurgica, 17(1):101 - 104, 1983.

[104] Schneider, R, B Heine, RJ Grant und Z Zouaoui: Mechanical behaviour of aircraft relevant aluminium wrought alloys at low temperatures. Proceedings of the Institution of Mechanical Engineers, Part L: Journal of Materials Design and Applications, 2013.

[105] Gao, Yajun, Yingqiang Fu, Wei Sun, Yinlu Sun, Hongbo Wang, FenGYIN WANG und JiANWEI ZHAO: Investigation on the mechanical behavior of fivefold twinned silver nanowires. Computational Materials Science, 55:322 - 328, 2012.

[106] Ding, W, DA Dikin, X Chen, RD Piner, RS Ruoff, E Zussman, X Wang und X LI: Mechanics of hydrogenated amorphous carbon deposits from electronbeam-induced deposition of a paraffin precursor. Journal of Applied Physics, 98(1):014905, Juli 2005.

[107] Utke, I., V. Friedli, S. Fahlbusch, S. Hoffmann, P. Hoffmann und J. MichleR: Tensile Strengths of Metal-Containing Joints Fabricated by Focused Electron Beam Induced Deposition. Adv. Eng. Mater., 8(3):155-157, 2006.

[108] Wu, Bin, Andreas Heidelberg und John J. Boland: Mechanical properties of ultrahigh-strength gold nanowires. Nat Mater, 4(7):525-529, Juli 2005.

[109] Roos, B., M. Seibt und C. Volkert: Private communication. 2008. 



\section{Danksagung}

An dieser Stelle möchten ich allen Leuten danken, die zum Gelingen dieser Arbeit beigetragen haben und mich in dieser Zeit begleitet haben.

An erster Stelle möchte ich mich bei Prof. Cynthia Volkert bedanken, die mir die Möglichkeit gegeben hat diese Arbeit anfertigen zu können. Weiterhin möchte ich ihr für die Betreuung, die Diskussionen und Gespräche während der gesamten Zeit danken sowie dafür, dass sie immer an das Gelingen dieser Arbeit und an mich geglaubt hat.

Prof. Michael Seibt danke ich für die Betreuung während der Doktorzeit sowie für die Übernahme des Koreferats. Außerdem danke ich Prof. Gunther Richter für die Bereitstellung der Proben.

Ein großes Dankeschön geht an alle aktuellen und ehemaligen Mitgliedern der Nano-Gruppe für ihre Hilfe, Diskussionen, Ideen und Anregungen. Besonders möchte ich mich bei Eike Epler bedanken für seine gesamte Hilfe sowie die enge Freundschaft, bei Burkhard Roos, der mir so vieles beigebracht hat und selbst nach seiner Zeit am IMP noch Zeit für Diskussionen hatte, und bei meinen Bürokollegen Kodanda Mangipudi und Sönke Schmidt, für die angenehme Zeit und die vielen kurzen und langen Diskurse.

Der E-Werkstatt, der Feinmechanik und den Technikern danke ich für die Unterstützung bei sämtlichen kleinen und größeren Angelegenheiten, die im Laufe der Zeit aufgetreten sind und immer sofort erledigt worden sind. Besonders möchte ich mich bei Tobias Schulz bedanken, der immer für jede Hilfe bereit stand und auch abseits der Arbeit viel für eine gute Zusammenarbeit getan hat, und bei Volker Radisch für sämtliche Hilfen mit den REMs sowie die gemeinsamen Anstrengungen, das MESSI einigermaßen in den Griff zu bekommen. Allen anderen Mitgliedern des IMP danke ich für die angenehme Atmosphäre am Institut und bei der Arbeit.

Ein ganz besonderer Dank gilt meinen Eltern. Ohne eure Unterstützung, Zuversicht und Hilfe wäre diese Arbeit nicht möglich gewesen.

Schließlich danke ich meiner zukünftigen Frau Anna für ihre Liebe und Unterstützung. Du warst immer für mich da. Wenn es mal nicht so lief, hast du mich wieder aufgebaut. Ohne dich hätte ich es nicht geschafft. Dir widme ich diese Arbeit. 
